Chemical Technology Division

Chemical Technology Division

Chemical Technology Division

Chemical Technology

\title{
Tests with Ceramic Waste Form Materials Made by Pressureless Consolidation
}

Division

Chemical Technology

Division

Chemical Technology by M. A. Lewis, M. C. Hash, Division

Chemical Technology

Division

Chemical Technology

Division

Chemical Technology

Division

Chemical Technology

Division

Chemical Technology

Division

Chemical Technology

Division

Chemical Technology

Division

Chemical Technology

Division

A. S. Hebden, and W. L. Ebert 
Argonne National Laboratory, with facilities in the states of Illinois and Idaho, is owned by the United States Government and operated by The University of Chicago under the provisions of a contract with the Department of Energy.

\begin{tabular}{l} 
DISCLAIMER \\
This report was prepared as an account of work sponsored by an agency of \\
the United States Government. Neither the United States Government nor \\
any agency thereof, nor The University of Chicago, nor any of their \\
employees or officers, makes any warranty, express or implied, or assumes \\
any legal liability or responsibility for the accuracy, completeness, or \\
usefulness of any information, apparatus, product, or process disclosed, or \\
represents that its use would not infringe privately owned rights. Reference \\
herein to any specific commercial product, process, or service by trade name, \\
trademark, manufacturer, or otherwise, does not necessarily constitute or \\
imply its endorsement, recommendation, or favoring by the United States \\
Government or any agency thereof. The views and opinions of document \\
authors expressed herein do not necessarily state or reflect those of the \\
United States Government or any agency thereof, Argonne National \\
Laboratory, or The University of Chicago. \\
\hline
\end{tabular}

Available electronically at http://www.doe.gov/bridge

Available for a processing fee to U.S. Department of Energy and its contractors, in paper, from:

U.S. Department of Energy

Office of Scientific and Technical Information

P.O. Box 62

Oak Ridge, TN 37831-0062

phone: (865) 576-8401

fax: (865) 576-5728

email: reports@adonis.osti.gov 
ANL-02/10

ARGONNE NATIONAL LABORATORY

9700 South Cass Avenue

Argonne, IL 60439

\title{
TESTS WITH CERAMIC WASTE FORM MATERIALS MADE BY PRESSURELESS CONSOLIDATION
}

\author{
by
}

M. A. Lewis, M. C. Hash, A. S. Hebden, and W. L. Ebert

Chemical Technology Division

September 2002 


\section{ACKNOWLEDGEMENTS}

Paul Johnson provided XRD analyses. Yifen Tsai and Susan Lopykinski provided ICPMS and ICP-AES analyses. Technical assistance was provided by Nicole Stephenson and Mark Clark. This work was performed as part of the U.S. Department of Energy's Electrometallurgical Treatment Program under the auspices of the DOE's Office of Nuclear Energy, Science and Technology under Contract W-31-109-Eng-38. 


\section{ACRONYMS}

AFSM

ASTM

CWF

DTD

EA glass

HIP

HIP CWF

ICP-AES

ICP-MS

MCC-1

$N L(i)$

$\mathrm{PC}$

PC CWF

PCT

RWS test

SEM

SLZ

T-t PC CWF

WASRD

XRD
Accessible free salt measurement

American Society for Testing and Materials

Ceramic waste form

Day-to-day

Environmental Assessment glass

Hot isostatic press, or hot isostatically pressed

Hot isostatically pressed ceramic waste form

Inductively coupled plasma-atomic emission spectroscopy

Inductively coupled plasma-mass spectrometry

Materials Characterization Center test method No. 1

Normalized elemental mass loss based on element $i$

Pressureless consolidation

Pressureless consolidated ceramic waste form

Product consistency test

Rapid water soluble test

Scanning electron microscopy

Salt-loaded zeolite

Temperature-time pressureless consolidated ceramic waste form

Waste Acceptance System Requirements Document

$\mathrm{X}$-Ray diffraction 


\section{CONTENTS}

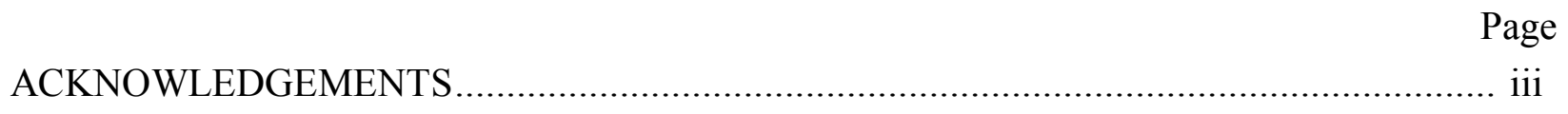

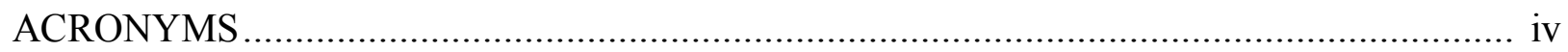

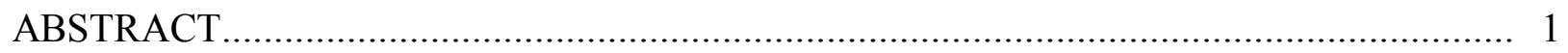

EXECUTIVE SUMMARY ................................................................................. 2

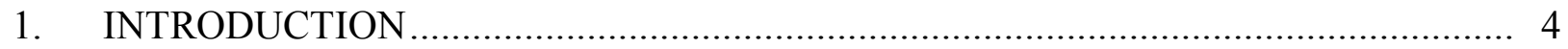

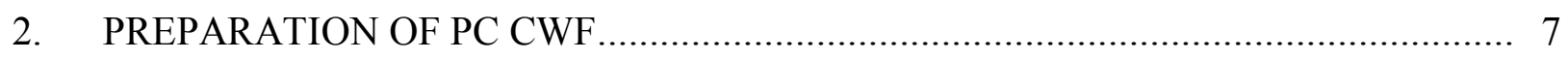

2.1 SALT PREPARATION .......................................................................... 7

2.2 ZEOLITE PREPARATION ................................................................. 7

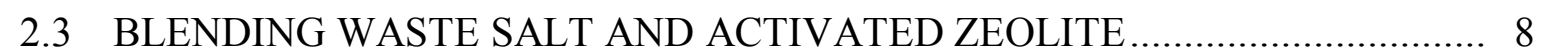

2.4 DRY MIXING SLZ AND GLASS ............................................................ 8

2.5 FABRICATING PC CWF MATERIALS .................................................... 8

3. CHARACTERIZATION OF PC CWF MATERIALS ............................................. 10

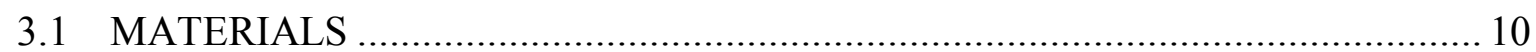

3.2 TEST METHODS.................................................................................... 12

3.2.1 Measurement of Bulk Density ......................................................... 12

3.2.2 Product Consistency Test with Rapid Water Soluble Test........................ 12

3.2.3 Accessible Free Salt Measurements ........................................................ 15

3.2.4 Static Test Method (MCC-1) .............................................................. 15

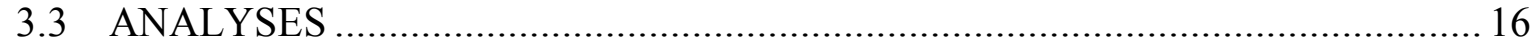

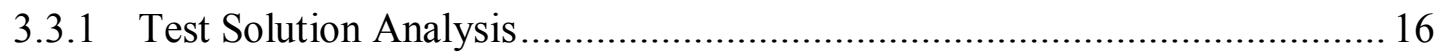

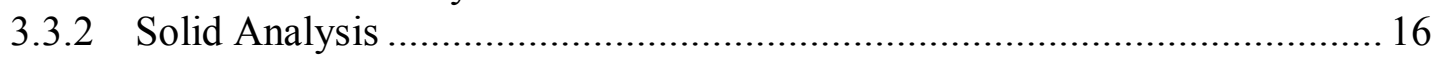

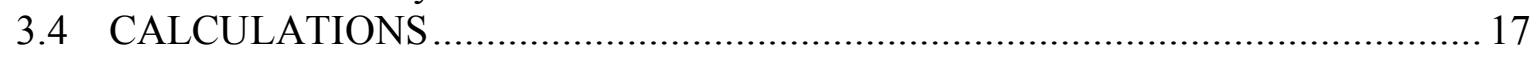

3.4.1 Normalized Elemental Mass Loss ........................................................... 17

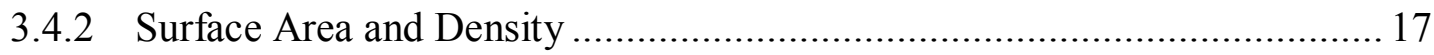

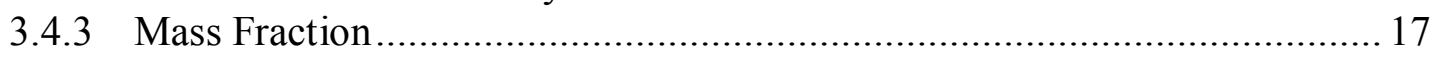

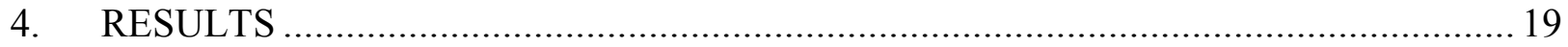

4.1 PHYSICAL PROPERTIES OF PC CWF PRODUCTS .................................. 19 


\section{CONTENTS (continued)}

4.1.1 Density............ 19

4.1.2 Elemental Concentrations in CWF Materials ........................................... 19

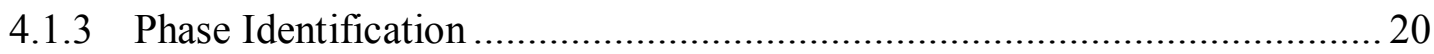

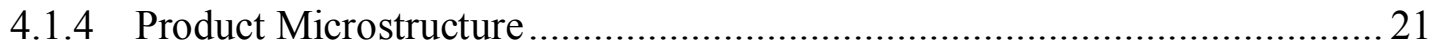

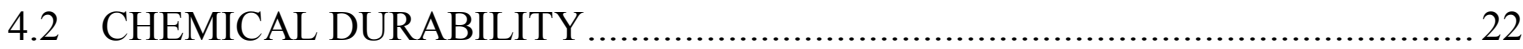

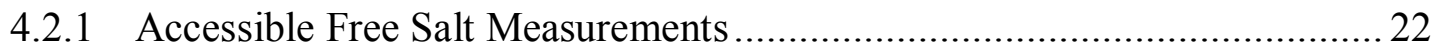

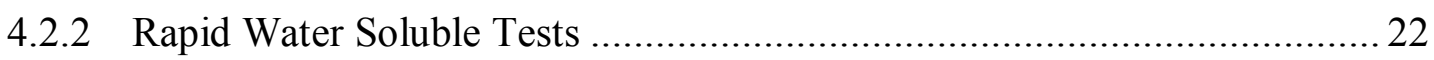

4.2.3 Comparison of MCC-1 Test Results for PC and HIP CWF ……………...... 23

4.3 SEVEN-DAY PRODUCT CONSISTENCY TESTS WITH VARIOUS

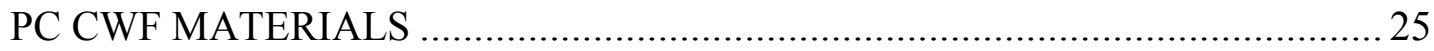

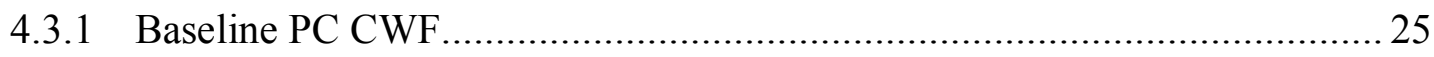

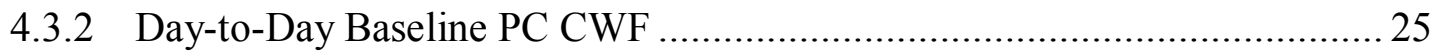

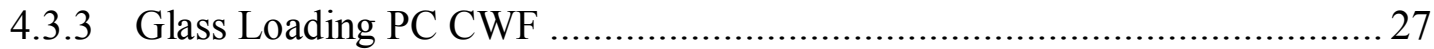

4.3.4 Tests with the Advanced PC CWF......................................................... 28

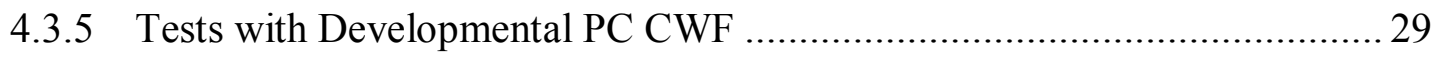

4.4 COMPARISON OF SEVEN-DAY PCT RESULTS ............................................. 30

4.4.1 Results for Different Products.................................................................. 30

4.4.2 Comparison of PCT Results for Different Laboratories................................. 31

4.4.3 Comparison of $N L$ (i) from PC CWF, HIP CWF and EA Glass..................... 32

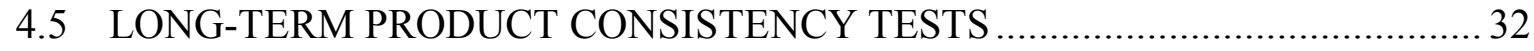

4.5.1 Long-Term PCTs with Baseline PC CWF....................................................... 33

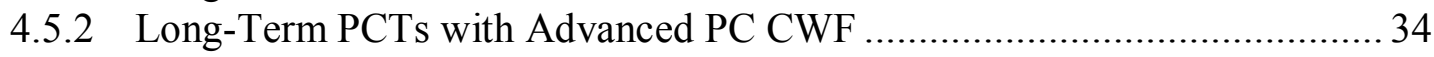

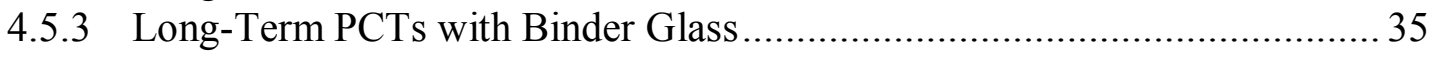

4.5.4 Apparent Solubility Limit of Orthosilicic Acid ............................................ 35

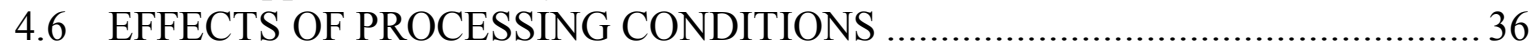

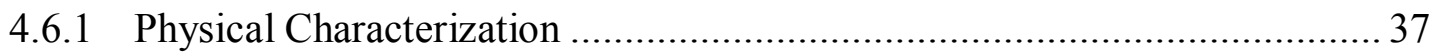

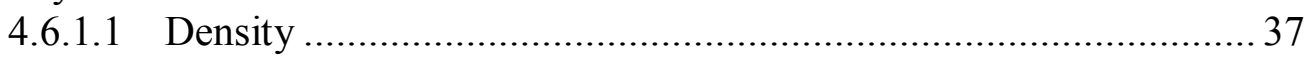

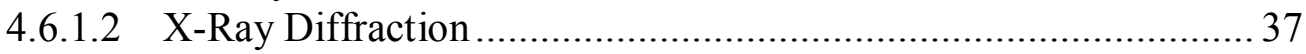

4.6.1.3 Scanning Electron Microscopy................................................... 37

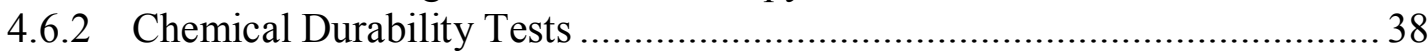

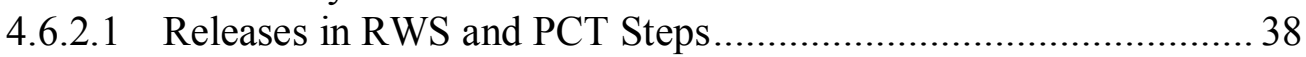

4.6.2.2 Halite Content from XRD …………………................................ 39

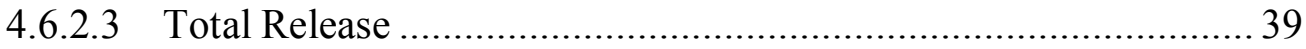

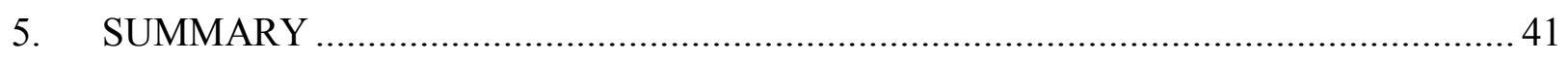

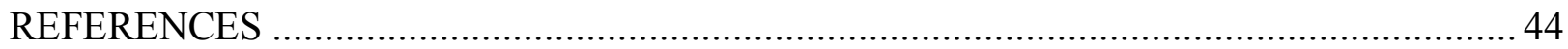




\section{TABLES}

1. Constituents of Simulated 300-Driver Salt ........................................................... 45

2. Measured Densities of Crushed and Bulk CWF Materials ........................................ 46

3. Average Compositions of Baseline PC, Advanced PC, HIP, and As-Batched CWF Materials

4. Measured Compositions of Different Sieve Fractions .......................................... 48

5. Results of XRD Analysis of PC CWF and HIP CWF Materials.............................. 49

6. Reference d-Spacings for Sodalite, Halite, and Nepheline ................................... 50

7. Results of AFSM with 5-kg Baseline PC CWF ................................................ 51

8. Results of RWS with 5-kg Baseline PC CWF ...................................................... 52

9. Results of MCC-1 Tests with PC CWF and HIP CWF ...................................... 53

10. Results of Triplicate Tests with 5-kg Baseline PC CWF ...................................... 54

11. Results of 7-Day PCT with DTD PC CWF Materials .......................................... 55

12. Results of PCT with Glass Loading PC CWF Materials ........................................ 56

13. Results of RWS with Advanced PC CWF Products............................................... 57

14. Results of 7-Day PCTs with Advanced PC CWF Materials .................................... 58

15. Concentrations in RWS Solutions in Filtrate Solutions........................................... 59

16. Results of RWS Tests with Developmental PC CWF Materials.............................. 60

17. XRD Results for Developmental PC CWF Materials .............................................. 61

18. Mean $N L(i)$ for CWF Products in Replicate 7-Day PCTs ...................................... 62

19. Results of PCT with Advanced PC CWF in Inter-laboratory Study ........................... 63

20. Statistics for PCT with Advanced PC CWF in Inter-laboratory Study ........................ 64

21. Statistics for PCTs with Advanced PC CWF and with Borosilicate Glasses ............... 65

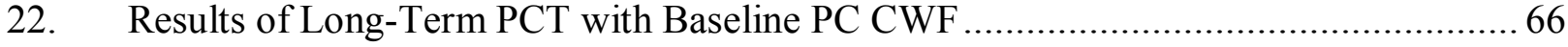




\section{TABLES (continued)}

23. Test Matrix for Long-Term PCTs with PC CWF and HIP CWF.................................6 67

24. Solution Concentrations in Long-Term PCTs with PC CWF and HIP CWF ..................68

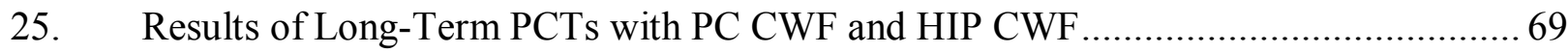

26. Test Matrix for Long-Term PCTs with Binder Glass................................................... 70

27. Solution Concentrations in Long-Term PCTs with Binder Glass .................................. 71

28. Results of Long-Term PCTs with Binder Glass and PC Glass ................................... 72

29. Orthosilicic Acid Concentrations and $\mathrm{pH}$ from Long-Term PCTs with

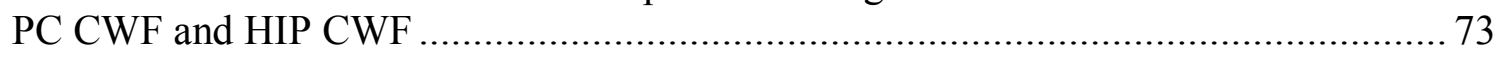

30. Orthosilicic Acid Concentrations and $\mathrm{pH}$ from Long-Term PCTs with Binder Glass.... 74

31. Processing Conditions for T-t PC CWF Products ………………............................ 75

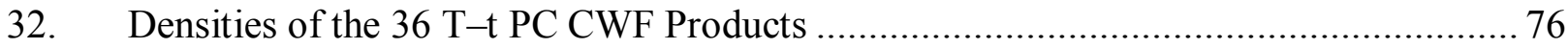

33. Measured d-Spacings for CWF Materials Processed for 1 Hour .................................... 77

34. Measured d-Spacings for CWF Materials Processed for 4 Hours................................... 78

35. Measured d-Spacings for CWF Materials Processed for 8 Hours................................. 79

36. Measured d-Spacings for CWF Materials Processed for 16 Hours.................................. 80

37. Measured d-Spacings for CWF Materials Processed for 24 Hours................................. 81

38. Measured d-Spacings for CWF Materials Processed for 36 Hours................................. 82

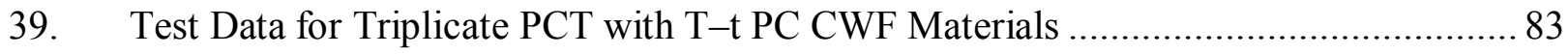

40. Results of Triplicate PCT with T-t PC CWF Materials Processed at $850^{\circ} \mathrm{C} \ldots \ldots \ldots \ldots \ldots . . . . .85$

41. Results of Triplicate PCT with T-t PC CWF Materials Processed at $875^{\circ} \mathrm{C}$..................8 86

42. Results of Triplicate PCT with T-t PC CWF Materials Processed at $900^{\circ} \mathrm{C} \ldots \ldots \ldots \ldots \ldots \ldots . .87$

43. Results of Triplicate PCT with T-t PC CWF Materials Processed at $915^{\circ} \mathrm{C} \ldots \ldots \ldots \ldots \ldots \ldots . . . . .88$ 


\section{TABLES (continued)}

44. Results of Triplicate PCT with T-t PC CWF Materials Processed at $925^{\circ} \mathrm{C} \ldots \ldots \ldots \ldots . . . . .89$

45. Results of Triplicate PCT with T-t PC CWF Materials Processed at $950^{\circ} \mathrm{C} \ldots \ldots \ldots \ldots . . . . . .90$

46. Results of PCT with T-t PC CWF Products ...................................................... 91 


\section{FIGURES}

1. SEM Image of Salt-Loaded Zeolite at Low Magnification and High

2. Photomicrographs Showing Microstructures of U and Pu-Doped PC CWF and $\mathrm{U}$ and Pu-Doped HIP CWF.

3. Scanning Electron Microscopy Photomicrographs of $-100+200$ Mesh Size Fraction

PC CWF before and after Subjection to Washing Procedure.

4. Photomicrographs Showing Surfaces of Baseline PC CWF and HIP CWF after 88-Day MCC-1 Test at $125^{\circ} \mathrm{C}$.

5. Mean Total $N L(i)$ for Three Sets of Triplicate 7-Day PCTs with the 5-kg Baseline PC CWF

6. Contributions to $N L(i)$ of RWS Solution and PCT Solution in Seven-Day PCTs with Replicate DTD Baseline PC CWF.

7. Summary Plot of Total NL(i) for Replicate DTD Baseline PC CWF Samples

8. Contributions to Concentration from RWS Solution and PCT Solution in Triplicate 7-Day PCTs with Glass Loading PC CWF.

9. Mean Concentrations in PCT Fraction of 7-Day PCTs with Glass Loading PC CWF.

10. Contribution to $N L(i)$ of RWS Solution and PCT Solution in 7-Day PCTs with Advanced PC CWF

11. Summary Plot of Total NL(i) in 7-Day PCTs for Advanced PC CWF Samples 105

12. Total $N L(i)$ in 7-Day PCTs with DTD and 5-kg Baseline PC CWF, 25\% Glass Loading PC CWF, Advanced PC CWF, and HIP CWF Products. 106

13. Mean Values of Solution Concentrations

14. Plot of $k$ Statistic Values and Critical Value $=1.98$ for PCTs with CWF 108

15. Plot of $h$ Statistic Values and Critical Value $= \pm 1.92$ for PCTs with CWF.

16. Mean $N L(i)$ for PCT Fractions of Long-Term PCTs with 5-kg Baseline PC CWF...... 110

17. $N L(i)$ for Long-Term Tests with Advanced PC CWF and HIP CWF 


\section{FIGURES (continued)}

18. $N L(i)$ for Long-Term Tests with PC Glass and Binder Glass

19. Orthosilicic Acid Concentrations in PCT with Advanced PC CWF and HIP

CWF, and PC Glass and Binder Glass.

20. SEM Image of T-t PC CWF Material NLS-2 Processed at $850^{\circ} \mathrm{C}$ with $1-\mathrm{h}$ Hold

Time.

21. SEM Image of T-t PC CWF Material NLS-8 Processed at $915^{\circ} \mathrm{C}$ with $16-\mathrm{h}$ Hold

Time. 116

22. $N L(i)$ Values for 7-Day PCTs with T-t PC CWF Made with 1-h Hold Time

23. $N L(i)$ Values for 7-Day PCTs with T-t PC CWF Made with 4-h Hold Time 118

24. $N L(i)$ Values for 7-Day PCTs with T-t PC CWF Made with 8-h Hold Time 119

25. $\quad N L(i)$ Values for 7-day PCTs with T-t PC CWF Made with 16-h Hold Time

26. $N L(i)$ Values for 7-day PCTs with T-t PC CWF Made with 24-h Hold Time

27. $\quad N L(i)$ Values for 7-day PCTs with T-t PC CWF Made with a 36-h Hold Time

28. Correlation between $N L(\mathrm{Cl}), N L(\mathrm{I})$, and $N L(\mathrm{Na})$ in RWS Fraction .123

29. Correlation between $\% \mathrm{Cl}$ in RWS and Mass\% Halite Measured by XRD for CWF ...... 124

30. PCT Results for T-t PC CWF Materials Made With Different Hold Times 125 


\title{
TESTS WITH CERAMIC WASTE FORM MATERIALS MADE BY PRESSURELESS CONSOLIDATION
}

\author{
M. A. Lewis, M. C. Hash, A. Hebden, and W. L. Ebert
}

\begin{abstract}
A multiphase waste form referred to as the ceramic waste form (CWF) will be used to immobilize radioactively contaminated salt wastes recovered after the electrometallurgical treatment of spent sodium-bonded nuclear fuel. The CWF is made by first occluding salt in zeolite and then encapsulating the zeolite in a borosilicate binder glass. A variety of surrogate CWF materials were made using pressureless consolidation (PC) methods for comparison with CWF consolidated using a hot isostatic press (HIP) method and to study the effects of glass/zeolite batching ratio and processing conditions on the physical and chemical properties of the resulting materials. The data summarized in this report will also be used to support qualification of the PC CWF for disposal in the proposed federal high-level radioactive waste repository at Yucca Mountain. The phase composition and microstructure of HIP CWF and PC CWF are essentially identical: both are composed of about $70 \%$ sodalite, $25 \%$ binder glass, and a $5 \%$ total of inclusion phases (halite, nepheline, and various oxides and silicates). The primary difference is that PC CWF materials have higher porosities than HIP CWFs. The product consistency test (PCT) that was initially developed to monitor homogeneous glass waste forms was used to measure the chemical durabilities of the CWF materials. Series of replicate tests with several PC CWF materials indicate that the PCT can be conducted with the same precision with CWF materials as with borosilicate glasses. Short-term (7-day) PCTs were used to evaluate the repeatability of making the PC CWF and the effects of the glass/zeolite mass ratio, process temperature, and processing time on the chemical durability. Long-term (up to 1 year) PCTs were used to compare the durabilities of HIP and PC CWFs and to estimate the apparent solubility limit for the PC CWF that is needed for modeling. The PC and HIP CWF materials had similar disabilities, based on the release of silicon in long-term tests. These tests and analyses indicate that CWF made using the PC and HIP methods should be equally acceptable for disposal. The same waste loading can be used in PC CWFs and HIP CWFs. The disposition of radionuclides is the same in PC and HIP CWFs. One minor difference is that radionulcide and halite inclusions are fairly uniformly distributed in the binder glass phase of the PC CWF, whereas they are segregated near the sodalite domains in the HIP CWF. This is an advantage of the PC CWF, since the aggregation of halite inclusion lowers the effective durability of the surrounding glass, due to the greater exposed glass surface area after the halite dissolves.
\end{abstract}




\section{EXECUTIVE SUMMARY}

The ceramic waste form (CWF) was developed to immobilize radioactive salt wastes recovered after electrometallurgical treatment of spent sodium-bonded nuclear fuels, such as those from the ANL Experimental Breeder Reactor-II (EBR-II) and the Fermi reactor. A homogeneous glass waste form similar to that used at other DOE sites is not amenable to these salt wastes because of the low solubility of chloride in borosilicate glasses. Therefore, the salt is first occluded in zeolite, then the salt-loaded zeolite (SLZ) is encapsulated in a borosilicate glass. During the encapsulation step, the salt-loaded zeolite transforms to the mineral sodalite and submicrometer-sized inclusions of halite (crystalline $\mathrm{NaCl}$ ) and rare earth and actinide oxides become fixed in the binder glass. A hot isostatic press (HIP) method was used for encapsulating the SLZ during the development of the CWF. In FY 2000, the decision was made to use a pressureless consolidation (PC) method instead of the HIP method. The work described in this report was conducted to compare the quality of the CWF materials made using the HIP and PC methods and to evaluate the effects of waste loadings and various processing conditions.

Several PC CWF materials were made with salts containing added $\mathrm{U}$ and $\mathrm{Pu}$ and with different glass/salt-loaded zeolite mass ratios to evaluate the effects of waste loading at different processing temperatures and hold times. These materials were analyzed with X-ray diffraction to identify and quantify crystalline phase assemblages, and their bulk densities were measured. The chemical durabilities of the PC CWF materials were measured using the Material Characterization Center No. 1 (MCC-1) static leach test and the product consistency test (PCT). Tests were conducted to measure the effects of product size and the uniformity of samples taken from different locations within a sample. Replicate products were made to measure the repeatability of the PC process with laboratory-scale products. Replicate tests were also conducted to measure the within-laboratory repeatability and the between-laboratory reproducibility of the PCT method with PC CWF materials.

Essentially the same phase assemblages are produced in HIP CWFs and PC CWFs, with only small differences in the amounts of minor phases. Both are composed of about $70 \%$ sodalite, $25 \%$ binder glass, and about a $5 \%$ total of halite and oxide inclusion phases. The disposition of radionuclides is the same in both materials: plutonium, uranium, and rare earth fission products are present in 50-nm size microcrystalline mixed oxide inclusions in the binder glass; iodine is distributed congruently with chloride in sodalite and halite; and cesium is believed to be primarily dissolved in the binder glass. The distribution of the inclusions differ slightly. The inclusions in the HIP CWF are segregated in glass near the sodalite domains, whereas the inclusions are more uniformly distributed in the PC CWF materials. The difference is attributed to differences in the fluidity of the binder glass during processing. The HIP CWF is processed at $850^{\circ} \mathrm{C}$ for about four hours, whereas the PC CWF is processed at higher temperatures and for longer times; the current baseline PC processing conditions are $915^{\circ} \mathrm{C}$ for 16 hours. The rare earth and actinide oxides are formed on the outer surface of the zeolite when it is loaded with salt. We believe small amounts of $\mathrm{NaCl}$ are expelled from the $\mathrm{SLZ}$ when it converts to sodalite and that small inclusions of molten halite form within the glass. The higher temperatures and longer processing times facilitate mixing of the inclusions and the binder glass in the PC CWF materials. Tests have shown that the glass at the interface with sodalite dissolves faster than bulk glass in both HIP CWF and PC CWF materials. 
The zeolite used to make the CWF is a clay-bound aggregate of $10-\mu \mathrm{m}$ sized polycrystalline grains of zeolite. The aggregates consist of about $100-\mu \mathrm{m}$ sized particles. After conversion, the sodalite retains the morphology of the zeolite, with glass infiltrating the small voids between the grains. Glass infiltrates the voids more quickly and completely under the high pressure used in the HIP consolidation method than at ambient pressure in the PC method. The PC CWF materials also contain macroscopic voids, which occupy about 10-30\% of the waste form volume. The bulk density of the HIP CWF is about $2.3 \mathrm{~g} / \mathrm{cm}^{3}$, whereas the bulk density of the PC CWF ranges from about 1.5 to $2.2 \mathrm{~g} / \mathrm{cm}^{3}$, depending on process conditions.

The glass/SLZ ratio used to make the CWF determines the waste loading. Both HIP and PC CWFs can be made with $25 \%$ glass $/ 75 \%$ SLZ. This is about the highest waste loading that can be achieved while completely encapsulating the sodalite in glass. This requires a processing time of about $8 \mathrm{~h}$ for the PC CWF. Both the density and chemical durability of the PC CWF increase as the processing temperature and time are increased, up to about $925^{\circ} \mathrm{C}$. Processing at higher temperatures results in separation of the glass and sodalite phases because the glass becomes too fluid. The amount of halite inclusions that form also increase with the processing temperature and time. This may be because the sodalite dissolves into the glass. A compromise between durability and halite content must be reached. The halite content is important because (1) iodine is released as the halite dissolves, and (2) chloride in solution attacks steel containers and the metallic waste form in co-disposal packages. (The metallic waste form is made from cladding hulls recovered from the electrorefiner.)

The 7-day PCT was used to compare the chemical durabilities of samples taken from different locations in a PC CWF product to assess the repeatability of making PC CWF products and measure the effects of the glass/SLZ ratio and processing conditions. The repeatability of the 7-day PCT was measured with replicate tests to gauge the sensitivity of the test to differences in the chemical durability of different materials. A short water-washing step at room temperature is part of the PCT procedure. We used the amount of chlorine dissolved in the water-wash step to quantify the amount of halite in the PC CWF material. This was shown to be proportional to the amount of halite in the bulk material as measured with X-ray diffraction. The boron released in the 7-day test provides a measure of the amount of binder glass that dissolved. The relative amounts of $\mathrm{B}$ and $\mathrm{Si}$ that are released provide insight into the relative amounts of binder glass and sodalite that have dissolved. The PCT responses of the HIP and PC CWF materials are both dominated by dissolution of binder glass. Dissolution of binder glass is also expected to dominate degradation of CWF materials in the disposal system. This is because the solution in the PCT quickly becomes saturated with sodalite, but remains undersaturated with respect to the binder glass. Similar behavior is expected in the disposal system. Thus, the PCT provides a useful measure of PC CWF durability in the disposal system.

The Waste Acceptance System Requirements Document (WASRD) requires that the chemical durability of glass waste forms be consistently better than that of a benchmark glass, the Environmental Assessment (EA) glass in a 7-day PCT. The durabilities of glass waste forms are tracked by the release of boron, alkali metals, and silicon. The durabilities of both the sodalite and binder glass phases in the PC CWF can also be tracked by these elements. Comparison of the PCT responses of the PC CWF and the EA glass show that the PC CWF will meet the consistency requirement in the WASRD. 


\section{INTRODUCTION}

A glass-bonded sodalite ceramic waste form (CWF) has been developed at Argonne National Laboratory (ANL) to immobilize the radioactive electrorefiner salt waste generated during electrometallurgical treatment of spent sodium-bonded nuclear fuel. Sodium and most of the fuel components are dissolved in the molten $\mathrm{LiCl}-\mathrm{KCl}$ eutectic salt electrolyte during treatment. Dissolved uranium is recovered at the cathode while sodium and most of the fission products and actinides remain dissolved in the electrolyte. Due to the buildup of radionuclides as the fuel dissolves, the salt must be removed from the electrorefiner periodically and disposed of as a high-level radioactive waste. Components of the fuel rods that do not dissolve (primarily the cladding hulls) are recovered and disposed of as a separate waste form. Borosilicate glass is the standard waste form for DOE high-level wastes. However, the very low solubility of chloride in borosilicate glass precludes direct vitrification of the waste salt. The CWF avoids the solubility limitation by first occluding the salt in zeolite, then encapsulating the salt-loaded zeolite in a borosilicate glass. As described in this report, the physical and chemical properties of the resulting waste form are similar to those of borosilicate glass waste forms, and the CWF is expected to be accepted for disposal in the proposed federal high-level waste repository at Yucca Mountain.

The CWF is made by first occluding the waste salt within the cage structure of zeolite $4 \mathrm{~A}$ by mixing the salt and zeolite at $500^{\circ} \mathrm{C}$ in the absence of water. The saltloaded zeolite (SLZ) is then mixed with the binder glass and heated to consolidate into a monolithic waste form. When heated above about $800^{\circ} \mathrm{C}$, the SLZ converts to the mineral sodalite, $\mathrm{Na}_{8}\left(\mathrm{AlSiO}_{4}\right)_{6} \mathrm{Cl}_{2}$. Trace amounts of nepheline, $\mathrm{NaAlSiO}_{4}$, may also be formed under some processing conditions. Most of the $\mathrm{NaCl}$ from the waste salt becomes incorporated into the sodalite structure. The small amount of salt that does not become incorporated into sodalite forms micrometer-size halite $(\mathrm{NaCl})$ inclusions in the binder glass. The resulting CWF is a multiphase material containing approximately 25 mass \% binder glass that encapsulates about 70 mass\% sodalite, and a 5 mass\% total of halite, nepheline, and mixed rare earth and actinide oxides and silicates. Radionuclides are distributed among several phases in the CWF. The actinide and rare earth element fission products are present as mixed oxide inclusions in the binder glass; alkali metal and alkaline earth fission products (e.g., Cs) are dissolved in the glass binder; and most of the iodine is fixed in the sodalite, although a small fraction of the iodine inventory is present in the halite crystals.

Two processing methods have been developed to consolidate the waste form into a monolith: hot isostatic press (HIP) and pressureless consolidation (PC). The CWF materials that result from the HIP and PC processes are composed of the same major phases and have nearly identical microstructures. The main difference between waste forms processed by the two methods is that the PC CWF has a lower bulk density than the HIP CWF - the bulk density of PC CWF materials can range between 1400 and $2200 \mathrm{~kg} / \mathrm{m}^{3}$ depending on the processing conditions, while the bulk density of HIP CWF materials is about $2330 \mathrm{~kg} / \mathrm{m}^{3}$. The lower density of the PC CWF materials is due to higher porosity. This includes pores remaining due to incomplete densification of the 
material and incomplete infiltration of glass into voids between grains in the sodalite granules. Since the same phases comprise the HIP CWF and PC CWF waste forms, the waste forms are expected to have similar chemical durabilities under disposal conditions.

Pressureless consolidation was recently selected as the preferred option for immobilization of sodium-bonded spent fuel from the Experimental Breeder Reactor-II (EBR-II) [1]. This decision was based primarily on the technical uncertainty of installing and operating a large hot isostatic press in the hot cell at the Argonne National Laboratory-West (ANL-W) facility where the electrometallurgical treatment will be conducted. Selection of the PC method as the preferred option was supported by preliminary tests conducted with the PC CWF to confirm that its qualification for disposal was not less likely than that of the HIP CWF. We expect that the insights regarding chemical durability gained from the many tests conducted with the HIP CWF can be used to understand and model the performance of the PC CWF. The results from testing and analysis of HIP CWF materials conducted during development of the CWF are expected to be part of the database used to qualify the PC CWF for disposal. Some of the tests conducted to confirm those expectations are described in this report. Additional tests with the PC CWF have been conducted to address the following needs: (1) determine the effects of process conditions on the microstructure and chemical durability of the waste form, (2) measure model parameter values needed to calculate long-term dissolution behavior under disposal conditions, and (3) measure the consistency with which waste forms can be produced by pressureless consolidation. The results of those tests and analyses are given in this report.

The report is organized as follows. The processing steps used to make PC CWF materials are described in Section 2. A variety of PC CWF materials have been made using different processing parameter values, such as the relative amount of salt, zeolite, and binder glass, processing temperature and time, and product size. Materials have been made to evaluate the sensitivity and reproducibility of test methods, study the degradation behavior, and measure model parameter values. These materials and the test and analysis methods used in these studies are described in Section 3. The test results are presented and discussed in Section 4 in three parts: studies of the general properties of CWF corrosion, studies with several different CWF materials, and studies of the effects of process conditions for a particular CWF composition. The results and discussion of the tests are organized according to the issues they address. The following issues were studied:

- The crystalline phases and microstructure of several PC CWF materials were characterized with X-ray diffraction (XRD) and scanning electron microscopy (SEM) with associated energy dispersive X-ray emission spectroscopy (EDS). These analyses showed that the same major phases of glass, sodalite, and halite occurred in PC CWFs made under a range of conditions. Trace amounts of nepheline and albite were seen to form under extreme conditions of temperature and hold time. 
- Dissolution behavior and consistency were studied using the Product Consistency Test (PCT) method and the Material Characterization Center No. 1 (MCC-1) static leach test. As a part of the PCT procedure with CWF materials, the water used to wash the crushed material before conducting the test was analyzed for $\mathrm{Cl}$ (and sometimes also for $\mathrm{Na}$ ) to provide a measure of the amount of halite $(\mathrm{NaCl})$ at the surface of the CWF. This analysis is referred to as the rapid water soluble (RWS) test. Replicate RWS tests were conducted to measure the repeatability of the test and the uniformity of the halite distributions in the CWF.

- The long-term dissolution behaviors of the PC CWF and HIP CWF materials under these test conditions were measured and compared. These results show that the dissolution behaviors of the PC CWF and HIP CWF are essentially the same, and that model parameter values measured using tests with the HIP CWF can be used to model the dissolution of PC CWF materials.

- The repeatability of the PCT conducted with the PC CWF was measured in replicate tests with well-homogenized materials taken from an individual PC $\mathrm{CWF}$ product. These results were used to distinguish test precision from differences in test responses caused by different products. These results show that PC CWF materials are homogeneous on the scale of the sample size used for the PCT.

- The reproducibility of making the PC CWF was measured by conducting PCTs with 10 products that were prepared using the same batch source of glass frit and salt-loaded zeolite and processed under the same conditions, but on 10 different days. These results show that the PC CWF can be made reproducibly, within the precision of the PCT.

- The intra-laboratory repeatability and inter-laboratory reproducibility of conducting a 7-day PCT with the PC CWF was measured in an interlaboratory study (ILS). Six independent data sets were produced by participants from ANL, Pacific Northwest National Laboratory (PNNL), and Savannah River Technology Center (SRTC). These data were evaluated statistically following an American Society for Testing and Materials (ASTM) standard procedure for comparison with the results of the ILS conducted with borosilicate glasses. These results show that the PCT can be conducted as precisely with the CWF as it can with borosilicate waste glasses.

- The effects of processing conditions on chemical durability were measured in tests with PC CWF materials prepared at different hold temperatures and different hold times, and with PC CWF products made with different glass/zeolite mass ratios. These results can be used to select processing conditions to optimize waste loading and densification while maintaining acceptable product performance. 


\section{PREPARATION OF PC CWF}

Preparation of the PC CWF involves the following steps: (1) preparing the waste salt, (2) drying the zeolite, (3) blending the zeolite and salt, (4) mixing the glass and saltloaded zeolite, and (5) pressureless consolidation. These steps are described below. Since both the waste salt and the zeolite are moisture-sensitive, care was taken to avoid exposure to humid air or water. This was accomplished by mixing under a purified argon ( $>99.999 \%)$ atmosphere. Many of the tests described in this report address the sensitivity of the waste form microstructure and chemical durability to the process parameters used in steps 4 and 5. We refer to the set of PC CWFs made using these same process conditions - including the relative amounts of SLZ and binder glass, the maximum processing temperature, and the hold time - as PC CWF materials. We refer to an individual $\mathrm{PC}$ CWF monolith, i.e., the piece recovered from a crucible, as a PC $\mathrm{CWF}$ product. A large number of PC CWF products of most PC CWF materials were made for testing and analysis.

\subsection{SALT PREPARATION}

Surrogate electrorefiner waste salt was prepared by mechanically mixing the constituents listed in Table 1, then melting the mixture at about $500^{\circ} \mathrm{C}$. The formulation in Table 1 is a nonradioactive surrogate for the salt chemistry expected in the electrorefiner after the electrometallurgical treatment of 300 driver assemblies, and is referred to as "simulated 300 driver salt." Mixtures of this composition were used to make all of the PC CWF materials described in this report. After heating for $16 \mathrm{~h}$, the molten salt was rapidly cooled by casting it onto a stainless steel pan. After it had cooled, the salt was crushed in a mortar and pestle and passed through a 325-mesh sieve to isolate particles with $<40 \mu \mathrm{m}$ average diameter. The mixing, melting, casting, cooling, and crushing were all performed in a glovebox having an argon atmosphere. The same sizing process will be used for actual waste salts.

\subsection{ZEOLITE PREPARATION}

Granulated Zeolite 4A with a nominal size of $-120+325$ mesh was supplied by UOP (Des Plaines, IL). The granules are aggregates of $<10-\mu \mathrm{m}$ size polycrystalline grains of zeolite that are held together with a clay binder. The as-received zeolite contained up to about $20 \%$ water. It was dried to remove all but about $0.2 \mathrm{wt} \%$ of the water to maximize the capacity of the zeolite for sorbing salt. A small amount of water was retained in the zeolite to avoid destroying its cage structure and salt-sorbing capacity. Slow heating and long hold times at progressively higher temperatures were used to gradually evaporate the water. Steam produced by heating the hydrated zeolite too rapidly can decompose the zeolite's crystalline structure. The heat treatment consisted of heating a $2-3 \mathrm{~kg}$ batch of zeolite $4 \mathrm{~A}$ stepwise for 20 -h periods in succession at about $150,200,350$, and $500^{\circ} \mathrm{C}$. The dehydrated zeolite is referred to as "activated zeolite." 


\subsection{BLENDING WASTE SALT AND ACTIVATED ZEOLITE}

The simulated waste salt and activated zeolite $4 \mathrm{~A}$ were mixed in a rotating dualcone vessel. The mixing vessel was hermetically sealed, removed from the glovebox, and installed in a furnace made of clamshell-styled heaters. These provided even, radially distributed heating. The blender was evacuated using a roughing pump for about $20 \mathrm{~min}$. It was then heated at about $5^{\circ} \mathrm{C} / \mathrm{min}$ to $500^{\circ} \mathrm{C}$ while being slowly rotated to continuously dry-mix the batch and uniformly heat the mixture. Once a temperature of $500^{\circ} \mathrm{C}$ was reached, the blender was evacuated with the roughing pump again, to remove any evolved gases, and held at $500^{\circ} \mathrm{C}$ for about $20 \mathrm{~h}$ while the salt became occluded in the zeolite. The furnace was then turned off and allowed to cool to room temperature with the mixing vessel. The blender was then removed from the heaters and returned to the purified argon atmosphere for further processing.

\subsection{DRY MIXING SLZ AND GLASS}

A borosilicate binder glass is added to bind the SLZ granules into a monolithic product. Blended SLZ is dry-mixed with coarse-grained glass at room temperature before thermal treatment. Glass with a nominal size of $-120+325$ mesh was supplied by PEMCO Corp. (Baltimore, MD). The similar size of the SLZ and binder glass facilitates mixing. In addition to homogenizing the mixture, dry mixing of the glass and SLZ at room temperature ensures safe handling and containment of the radioactive components that are present in some of the waste salts used for testing, and is done for processing actual waste salt. The initial development of the PC CWF was done using a mixture of $50 \%$ SLZ and $50 \%$ binder glass. The proportion of SLZ was subsequently increased to the current mixture of $75 \%$ SLZ and $25 \%$ binder glass to increase the waste salt loading in the CWF.

\subsection{FABRICATING PC CWF MATERIALS}

A range of crucibles sizes was used to make PC CWF products from between 20 $\mathrm{g}$ and $26 \mathrm{~kg}$ for testing. The dry-mixed SLZ and glass were poured and tapped into graphite crucibles to minimize the number of voids. The mixtures were heated in a programmable muffle furnace housed within a glovebox under a purified argon atmosphere. All specimens were heated at about $5^{\circ} \mathrm{C} / \mathrm{min}$. Materials made for this study were processed at temperatures ranging from 850 to $950^{\circ} \mathrm{C}$ and for hold times ranging from 1 to $36 \mathrm{~h}$, with the exception of the developmental PC CWF materials, which were heated at a higher rate and held at temperature for up to $168 \mathrm{~h}$.

A representative image of SLZ is shown in Figure 1. The low-magnification image in Figure 1a shows the agglomeration of $<10-\mu \mathrm{m}$ size zeolite grains into a 100$\mu \mathrm{m}$ size granule. The granule has been fixed in epoxy and cross-sectioned. Epoxy has filled the voids between the zeolite grains. The high-magnification image in Figure $1 \mathrm{~b}$ shows the accumulation of rare earth oxide precipitates at the outer surface of the SLZ granule. Rare earth and actinide oxides were seen only rarely within the granule. A few 
halite crystallites are also seen in Figure 1b. These probably formed on top of the cross section during sample preparation.

Polished cross sections of typical HIP CWF and PC CWF materials are shown in Figure 2. Samples of U,Pu-loaded CWF are shown. Note that the regions of sodalite are similar in size and shape to SLZ granules. The microstructure is discussed in more detail in Section 4.1.4. 


\section{CHARACTERIZATION OF PC CWF MATERIALS}

\subsection{MATERIALS}

A variety of PC CWF materials were prepared for testing and analysis to address various waste form performance issues and determine if the PC method could be used to produce acceptable waste forms. As a part of this study, PC CWF products were prepared under several conditions and with several different SLZ/glass ratios to measure the effects of salt waste loading and processing conditions on the microstructure and chemical durability of the PC CWF products. The different types of PC CWF products that were studied are summarized below with regard to the process conditions under which they were made, the tests and analyses conducted with those products, and the information provided by those tests.

- Baseline PC CWF. These were the first PC CWF products produced for proof-of-principle purposes for the PC process. They were made with 50 mass \% glass and 50 mass\% SLZ and were heated at $850^{\circ} \mathrm{C}$ for $4 \mathrm{~h}$. Samples taken from a $5-\mathrm{kg}$ product were used in replicate 7-day and long-term PCTs. The replicate 7-day tests were conducted to measure the repeatability of the PCT with the PC CWF materials (intra-laboratory precision), and the longterm tests were conducted to study the corrosion behavior for comparison with that of HIP CWF materials. In addition, one 20 -g product was produced on each of 10 different days from a mixture of glass and salt-loaded zeolite that was nominally the same as that used for the $5 \mathrm{~kg}$ product. These are referred to as replicate day-to-day (DTD) products, DTD-1 through DTD-10. Samples of these products were subjected to 7-day PCTs to measure the repeatability of producing PC CWF materials.

- Glass Loading PC CWF. Several products were made to study the effect of the mass ratio of glass and SLZ on the bulk density, microstructure, and chemical durability. Products were made with $25,30,35,40$, or 45 mass $\%$ glass and were heated at $850^{\circ} \mathrm{C}$ for $4 \mathrm{~h}$. Samples of these products were subjected to 7-day PCTs to evaluate the impact of waste loading on the quality of the waste form. The results were also used to assess the sensitivity of the PCT to the SLZ/glass ratio.

- Developmental PC CWF. These materials were made to evaluate the interaction between the glass and sodalite during consolidation of the waste form. Products were made with 50 mass $\%$ glass and 50 mass $\%$ SLZ and were heated at $850^{\circ} \mathrm{C}$ for 4,72 , and $168 \mathrm{~h}$ to study the effect of hold time on the size and abundance of the sodalite domains and the dissolution of the sodalite into the glass. These are referred to as developmental products PC-4, PC-72, and PC-168, respectively. Samples of these products were analyzed with SEM/EDS and XRD. 
- U,Pu-Doped PC CWF. Products were made with salt doped with plutonium and uranium to examine the distribution of those elements in the waste form and their release as the waste form corrodes. Materials were made with 25 mass \% binder glass and 75 mass $\%$ SLZ by pressureless consolidation $\left(875^{\circ} \mathrm{C}\right.$ for $24 \mathrm{~h}$ ). Samples of these products were subjected to 7-day PCTs for comparison with $\mathrm{PC}$ CWF products made without $\mathrm{U}$ and $\mathrm{Pu}$ and for longer durations to study the release behaviors of $\mathrm{U}$ and $\mathrm{Pu}$ as the PC CWF corrodes. Releases of matrix components in 7-day PCTs are compared with releases from nonradioactive CWF in this report. The complete results of long-term PCTs with these materials are presented elsewhere [2]. The results of the 7day PCTs are included in this report to show the combined effect of the presence of $\mathrm{U}$ and $\mathrm{Pu}$ in the $\mathrm{CWF}$ and processing conditions.

- Advanced PC CWF. The advanced PC CWF materials were made with 25 mass \% glass and 75 mass\% SLZ and were consolidated at $915^{\circ} \mathrm{C}$ for either 16 or $24 \mathrm{~h}$. The higher consolidation temperature, relative to the temperature used to process the baseline PC CWF, was used to achieve a higher bulk density. These materials are referred to as "advanced" because the waste loading and waste form density are higher than for the baseline PC CWF. Three 20 -g products were made to evaluate the repeatability of the process; $500-\mathrm{g}$ and $26-\mathrm{kg}$ products were made to study the effects of scale up; one $500-\mathrm{g}$ product was consolidated at $915^{\circ} \mathrm{C}$ for $16 \mathrm{~h}$ to determine if the hold time affected chemical durability. (The effects of temperature and hold time were later studied in greater detail using the T-t PC CWF materials; see below). Samples of the various advanced PC CW products were subjected to the RWS test to measure the abundance of halite inclusions. Samples of two products were subjected to 7-day PCTs to determine chemical durability.

- T-t PC CWF. A suite of 36 PC CWF materials was made with 25 mass\% glass and 75 mass\% SLZ at processing temperatures of 850, 875, 900, 915, 925 , and $950^{\circ} \mathrm{C}$ and processing times of $1,4,8,16,24$, and $36 \mathrm{~h}$. Two products were made for each temperature-time combination. One product was analyzed to determine the effect of processing temperature and time on the microstructure and density, and the other was tested to measure the chemical durability with 7-day PCTs. These materials are referred to as $\mathrm{T}-\mathrm{t}$ PC CWF products ("T-t" for "Temperature-time").

- Binder Glass. Several series of tests were conducted with the crushed binder glass received from the vendor. The glass was sieved to isolate desired size fractions for testing and washed to remove fines. These tests were conducted to compare the dissolution behavior of the glass phase in the CWF with that of pure glass. Some glass samples were made by remelting the binder glass at the same temperature and hold time used to make PC CWF materials. These are referred to as PC glasses. Some tests were conducted with PC glasses made at $915^{\circ} \mathrm{C}$ to compare the dissolution behavior of pure glass with the dissolution behavior of the glass phase in the PC CWF. 


\subsection{TEST METHODS}

\subsubsection{Measurement of Bulk Density}

A buoyancy method based on Archimedes' principle was used to measure both the bulk density of the PC CWF materials and the densities of the crushed PC CWF materials. The density is determined from the difference in the weight of a sample when in air and when suspended in a fluid of known density. Liquid octanol (density $=0.827$ $\mathrm{g} / \mathrm{mL}$ ) or demineralized water (density $=1.000 \mathrm{~g} / \mathrm{mL}$ ) was used as the suspension fluid. The bulk density of samples suspended in octanol was calculated by using Eq. 1a and the bulk density of samples suspended in water was calculated by using Eq. 1b:

$$
\begin{aligned}
& \rho_{b}=m_{D} \cdot 0.837 /\left(m_{S}-m_{S S}\right) \\
& \rho_{b}=m_{D} \cdot 1.000 /\left(m_{S}-m_{S S}\right)
\end{aligned}
$$

where $\rho_{b}$ is the bulk density, $m_{D}$ is the dry mass of the sample, $m_{S}$ is the mass of the sample saturated with octanol or water, and $m_{S S}$ is the mass of the sample when suspended in octanol or water, respectively.

\subsubsection{Product Consistency Test with Rapid Water Soluble Test}

The PCT has been standardized by the American Society for Testing and Materials (ASTM) as standard test method C1285 for evaluating the chemical durabilities of glass waste forms [3]. That standard includes two methods: PCT Method A and PCT Method B. PCT Method A was developed "to obtain data to evaluate whether the durability of waste glasses have been consistently controlled during production" [3]. PCT Method A is used to meet the product consistency requirement for borosilicate waste forms in the Waste Acceptance System Product Requirements Document (WASRD) [4]. The values of all test parameters specified for PCT Method A are: crushed glass in the size fraction $-100+200$ mesh $(0.149-0.074 \mathrm{~mm})$ washed to remove fines; demineralized water (ASTM Type I); glass/water mass ratio of 1:10; test temperature of $90 \pm 2{ }^{\circ} \mathrm{C}$; test duration of seven days; Type 304L stainless steel vessels; air atmosphere. PCT Method A requires that tests with the waste glass be run in triplicate and that duplicate blank tests and triplicate tests with a standard glass be conducted in parallel with tests with the waste glass. The blank tests are conducted to confirm that the test vessels were properly cleaned, and the tests with standard glass are conducted to confirm that the test was executed properly.

PCT Method B allows use of other parameter values and does not require parallel or blank tests to be run with standard glass. It was developed to provide data that could be used in the prediction of long-term glass corrosion behavior.

The preparation of crushed glass for testing is the same for both PCT methods. The procedure includes steps to wash the crushed glass to remove adhering fines prior to testing. In the procedure, it is recognized that some glasses may contain soluble phases that could dissolve during the washing process. The procedure recommends that these 
soluble phases be identified and that their dissolution rates relative to the bulk glass be determined. If preferential dissolution of such phases is likely to occur when the sample is washed with water, the PCT procedure provides the option of either analyzing the wash solution directly or omitting the water wash step and washing only with ethanol to remove fines. If the water wash is omitted, water-soluble phases will dissolve in the test solution and be included in the analysis of the test solution at the end of the test. If a water-soluble phase contains the same elements as the host glass phase, it may not be possible to distinguish the releases from the two phases.

Tests and analyses conducted previously with the HIP CWF revealed the presence of halite $(\mathrm{NaCl})$ inclusions in the binder glass that were very soluble in water. The PCT sample preparation procedure that was used with the PC CWF was slightly modified from that used with the HIP CWF to provide insight into the amounts of halite inclusions in the various PC CWFs as a part of the PCT procedure. For the PCT with the HIP CWF, the crushed material was washed repeatedly with absolute ethanol to remove fines. It was not washed with water. In the tests with the PC CWF, a separate water wash was conducted and the wash solution was analyzed for dissolved $\mathrm{Cl}$ to provide a measure of the abundance of halite in the waste form. This information can be used to confirm process control. This also provides a measure of the amounts of radioactive Cs and I contained in the halite, which is needed for modeling. The water wash method that is used for the PC CWF is referred to as the RWS test, and the water wash solution is referred to as the RWS solution. The elements that are released during the RWS step are referred to as being in the RWS fraction.

The PC CWF material was prepared for use in the PCT by crushing and dry sieving the $-100+200$ mesh size fraction. This is the fraction that passes through a 100 -mesh sieve (150- $\mu$ m opening) but is retained by a 200 -mesh sieve ( $74-\mu \mathrm{m}$ opening). The $-100+200$ mesh-size fraction was passed over the 200-mesh sieve a second time to remove some of the larger fines, then transferred to a beaker and washed according to the following procedure: (1) The crushed material was first subjected to an absolute ethanol wash. The ethanol was vigorously squirted onto the crushed material and the suspended CWF was swirled for about $30 \mathrm{~s}$. The ethanol-to-glass volume ratio was about 10:1. The ethanol and suspended fines were decanted immediately, i.e., there was no settling period. The crushed material was then oven-dried at $40^{\circ} \mathrm{C}$ and weighed. (2) The ethanol-washed material was then subjected to a 2-min ultrasonic water wash with $10 \mathrm{~mL}$ of demineralized water used per gram of crushed material. (3) After the water wash, the solution was decanted and then passed through a $450-\mathrm{nm}$ pore-size syringe filter. The resulting filtrate solution is referred to as the rapid water-soluble (RWS) solution. (4) The RWS solution was analyzed for $\mathrm{Cl}$ to provide a measure of the amount of halite that dissolved. For most scoping tests, the concentrations of components present in the glass and sodalite phases were measured in the RWS solution, as well. This was done to verify that dissolution of other phases in the CWF during the RWS step was negligible. It also provided an indication of other elements present in the halite phases, most notably I. The release of radionuclides during the dissolution of halite must be taken into account in performance assessment calculations. 
The initial wash with absolute ethanol is intended to remove most of the fines. Halite is not very soluble in absolute ethanol, so this wash removes only halite that is associated with fines. The water wash is intended to dissolve the halite phases (and other highly soluble phases that may be present in the CWF) that are exposed at the surface of the crushed CWF. Figures $3 \mathrm{a}$ and $3 \mathrm{~b}$ are SEM photomicrographs of crushed PC CWF before washing. Figures $3 \mathrm{c}$ and $3 \mathrm{~d}$ show the same PC CWF materials after washing once with ethanol and executing the RWS wash. Although an abundance of fines are seen to adhere to the crushed CWF prior to washing (Figs. 3a and 3b), none are present on the washed material (Figs. 3c and 3d). These photomicrographs show that the wash procedure effectively removes fines from crushed PC CWF materials. The low-magnification micrographs in Figs. $3 a$ and $3 c$ show the typical size and shape of crushed CWF in the $-100+200$ mesh size fraction.

It is important to note that analysis of the RWS solution provides a measure of the relative abundance of halite in the CWF, not the absolute abundance. This is because the halite inclusions that become exposed at the surfaces during crushing may penetrate to various depths into the waste form. For example, a particle of crushed CWF material can be modeled as a sphere with a diameter of $112 \mu \mathrm{m}$, which is the arithmetic average of the 100- and 200-mesh sieve openings. If it is assumed that the inclusions penetrate an average of $1 \mu \mathrm{m}$ into a spherical particle of crushed PC CWF, the halite that dissolves in the RWS comes from less than $3 \%$ of the particle volume. Later in this report, we compare the results of RWS with XRD results to show the correlation between the RWS response and the total amount of halite in the waste form.

The dried crushed material was used in the PCT. For clarity, we refer to the part of the PCT in which the material is reacted with water at $90^{\circ} \mathrm{C}$ for seven days as the PCT step to distinguish it from the RWS step; both steps are part of PCT Method A and PCT Method B. As discussed later in this report, the overall response of the PC CWF in PCT Method A or Method B is the sum of the responses in the RWS and PCT steps.

All PCTs were conducted with demineralized water in Teflon vessels. (The use of Teflon vessels makes these PCT Method B tests.) About $1 \mathrm{~g}$ of the PC CWF was added to the vessel, then a mass of demineralized water 10 times the mass of the PC CWF was added. The mass ratio of demineralized water to PC CWF sample was about 10:1 in most tests. Some of the long-term tests (i.e., tests conducted for longer than 7 days) were conducted at a mass ratio of 1:1 (using equal masses of PC CWF and demineralized water) to accelerate the saturation of the test solution. These tests were used to provide an estimate of the apparent saturation concentration of dissolved silica, which was needed for modeling. The filled and sealed vessels were placed in secondary containers, which were then partially filled with demineralized water and sealed. This was done to maintain similar water vapor pressures on the inside and outside of the Teflon test vessels to minimize the loss of water from the vessels during the test. The secondary containers were then placed into a $90^{\circ} \mathrm{C}$ oven. The oven temperature was set using a certified thermometer before the tests were started and continuously monitored using a thermocouple to confirm that the temperature remained within $2{ }^{\circ} \mathrm{C}$ of $90^{\circ} \mathrm{C}$, as required by the PCT procedure. 
Most tests were run for seven days to compare the test responses for different materials. A few tests were run for longer times to compare the long-term degradation of PC CWF materials with the long-term degradation of HIP CWF materials that had been measured previously. Those test results were also used to determine the apparent saturation concentration of dissolved silica for PC CWF materials. For the long-term PCT, water was added to a secondary container as needed to replace water lost to evaporation. At the completion of the test, the test vessel was removed from the secondary container and the outside of the vessel was dried with a paper towel. The vessel was then weighed and opened. Solution was removed from the vessel with a pipette and passed through a 450-nm pore-size syringe filter. The filtrate solution, which is referred to as the PCT solution, was submitted for analysis. The reacted glass was archived. A few samples of reacted glass were examined with SEM.

\subsubsection{Accessible Free Salt Measurements}

The abundance of soluble halite in the PC CWF was evaluated following the socalled "accessible free salt measurement (AFSM)" procedure that was developed at ANL. The AFSM was used previously to track the amount of halite in HIP CWF products prior to development of the RWS procedure. Briefly, the AFSM is conducted using the $-200+325$ size fraction of crushed material that is produced as a by-product of crushing PCT samples. An ethanol wash to remove fines is performed first, then two sequential water washes are performed. Typically about $1 \mathrm{~g}$ of crushed CWF is washed with two $10-\mathrm{mL}$ aliquots of demineralized water. The water wash solutions are combined and analyzed for chloride concentration. The results are presented as either the percent of the total chloride released to solution or as the normalized chloride mass loss, $N L(\mathrm{Cl})$ (see Section 3.4.1). The amount of chloride in the PC CWF sample is calculated from the sample mass and the mass fraction of $\mathrm{Cl}$ in the as-batched $\mathrm{CWF}$. The RWS is now used instead of the AFSM because it is more efficient (preparation of a separate size fraction is not required) and because it is consistent with steps that are already in the PCT procedure. This is important because the ASTM PCT procedure will be used to track the consistency of PC CWF products as part of the waste form qualification process. Several AFSMs were done with baseline PC CWF materials to (1) compare the AFSM responses of the PC CWF with those of the HIP CWF and (2) compare the AFSM responses of the PC CWF with the responses in the RWS procedure.

\subsubsection{Static Test Method (MCC-1)}

A few experiments were conducted following the static test method standardized by the ASTM method as test C1220 [5]. This method is essentially the same as the Materials Characterization Center No. 1 (MCC-1) test method, and these tests are referred to as MCC-1 tests in this report, for convenience. Samples were prepared for MCC-1 tests as discs approximately $2-\mathrm{mm}$ thick cut from 1.1-cm diameter cores. The faces of the samples were polished to a 600-grit final finish. Some samples were cut and polished without a cutting fluid to minimize the loss of halite during sample preparation. Others were prepared using absolute ethanol or water as a lubricant. One- and 3-day MCC-1 tests were conducted with samples from the 5-kg baseline PC CWF product. 
These tests were run at $90^{\circ} \mathrm{C}$ in 30 -mL Teflon vessels. Enough demineralized water was added to the vessel to achieve a CWF surface area-to-water volume $(S / V)$ ratio of $10 \mathrm{~m}^{-1}$. The geometric surface area was calculated by modeling the discs as right cylinders. These tests were conducted for comparison to tests with the HIP CWF that were conducted previously under the same conditions. Other tests were run with discs that were cut (using ethanol as a cutting fluid) from cores of the 5-kg baseline product and from a 50 -g product of the same composition. The faces of the discs were polished to a 600 -grit finish with water lubrication because the objective of those tests was to determine if there was preferential dissolution of binder glass relative to sodalite, and the loss of halite during sample preparation was not important. The tests were run at $125^{\circ} \mathrm{C}$ in 22-mL titanium vessels (Parr model 4701) at an $S / V$ ratio of about $8 \mathrm{~m}^{-1}$ for 28 and 88 days. These tests were conducted to accelerate dissolution relative to tests at $90^{\circ} \mathrm{C}$. The MCC-1 test conditions result in a greater alteration of the CWF surface than occurs in the PCT because much more material must dissolve in the MCC-1 test before solution feedback effects slow the reaction significantly.

\subsection{ANALYSES}

\subsubsection{Test Solution Analysis}

Aliquots of the test solutions were analyzed for $\mathrm{Cl}$ and I without further treatment. The chloride concentration was measured with a chloride ion selective electrode (ISE), and the iodide concentration was determined with inductively coupled plasma-mass spectrometry (ICP-MS). Solution aliquots taken for analysis of cations were stabilized with nitric acid and then analyzed with either ICP-MS or inductively coupled plasma-atomic emission spectroscopy (ICP-AES). The Cs concentrations were always measured with ICP-MS. Significant dilution of most PCT test solutions was required prior to ICP-MS analysis to avoid saturating the detector.

The solution $\mathrm{pH}$ was measured at room temperature using a combination electrode. The electrode/meter combination was calibrated using commercial buffer standards prior to use. The ISE/meter combination was calibrated before use with a series of standards made by serial dilution of a commercial $\mathrm{Cl}$ standard. The ICP-MS and ICP-AES systems were calibrated before use using commercial mixed standards.

\subsubsection{Solids Analysis}

Some CWF materials were examined using SEM with associated EDS to characterize the microstructure, detect fines, identify alteration phases, characterize changes in the surface morphology, etc. Monolithic samples were simply taped to aluminum sample stubs, and crushed samples were dispersed on double-sided tape. Some samples were fixed in epoxy then cut and polished to produce a cross section for examination. All specimens were coated with a thin film of carbon prior to SEM examination to increase electrical conductivity. 


\subsection{CALCULATIONS}

\subsubsection{Normalized Elemental Mass Loss}

The concentrations of several elements measured in the test solutions were used to calculate the normalized elemental mass loss, $N L$, which is given by the formula in Eq. (2)

$$
N L(i)=\left[C(i)-C^{\circ}(i)\right] /[(S / V) \cdot f(i)]
$$

where the $N L(i)$ is the normalized mass loss for element $i$; $C(i)$ is the concentration of element $i$ in the test solution; $C^{\circ}(i)$ is the background concentration of element $i$ (from the blank test); $S$ is the surface area of the crushed material; $V$ is the solution volume; and $f(i)$ is the as-batched mass fraction of element $i$ in the CWF. The surface area of crushed PC CWF was calculated as the product of the specific surface area times the mass of material used in the test. The specific surface area was calculated based on the size fraction and the density of the crushed material (see Section 3.4.2). The dimensions of the discs used in the MCC-1 tests were measured with calipers and used to calculate the geometric surface area for a right cylinder. The contributions of the RWS and PCT fractions to $N L(i)$ were calculated separately. Note that the value of $N L(i)$ is the mass of CWF dissolved per unit area, not the mass of element $i$. The values of $N L(i)$ that are calculated using the solution concentrations of different elements and for tests conducted with different CWFs, and at different $S / V$ ratios, can be compared directly because of normalization to the mass fraction of element $i$ in the dissolving solid and to the surface area, respectively.

\subsubsection{Surface Area and Density}

For calculation of $N L(i)$ from PCT results, the surface area, $S$ in Eq. (2), was calculated with the method used for particles of crushed material of a particular sieve size fraction [3]. Particles were modeled to be spheres with an average diameter equal to the arithmetic average of the sieve mesh opening sizes. For particles in the -100 +200 mesh size fraction, the average diameter is $0.112 \times 10^{-3} \mathrm{~m}$. The surface area of the sphere is $\pi d^{2}=3.94 \times 10^{-8} \mathrm{~m}^{2}$, and the volume is $\pi d^{3} / 6=7.36 \times 10^{-13} \mathrm{~m}^{3}$. The density of crushed HIP CWF is $2350 \mathrm{~kg} / \mathrm{m}^{3}$ (see Section 4.1.1), so the mass of a single particle is $1.72 \times 10^{-9} \mathrm{~kg}$, and the specific surface area is $22.9 \mathrm{~m}^{2} / \mathrm{kg}$ (which is $0.0229 \mathrm{~m}^{2} / \mathrm{g}$ ). The surface area of crushed material in a test is the product of its specific surface area and the mass used in the test.

\subsubsection{Mass Fraction}

The elemental mass fractions in the CWF materials, $f(i)$ in Eq. 2, represent the gross mass fractions, that is, the mass fractions in the CWF rather than the mass fractions in the individual phases. Even though an element like B is present only in the binder glass, its mass fraction is expressed in terms of the mass of CWF, that is, as $\mathrm{g} \mathrm{B} / \mathrm{g}$ $\mathrm{CWF}$. This is consistent with using the total surface area of the CWF to calculate $N L(i)$. 
Because the proportion of binder glass in the exposed surface area fraction of each phase is the same as the proportion of binder glass in the volume fraction, the proportion factor of binder glass in the CWF cancels in taking the product of $S \cdot f(i)$ in Eq. 2. That is, binder glass comprises $25 \%$ of the exposed CWF surface and $25 \%$ of the CWF mass. Likewise for other elements, the measured concentration, $C(i)$ in Eq. 2, is due to dissolution from the entire CWF surface, and the values of $S$ and $f(i)$ are for the entire CWF. 


\section{RESULTS}

\subsection{PHYSICAL PROPERTIES OF PC CWF PRODUCTS}

\subsubsection{Density}

The measured densities of the crushed CWF materials are summarized in Table 2. There are no differences in the densities of different size fractions of a given PC CWF material and no differences in the densities of the different PC CWF materials. The overall average density for all PC CWF materials in all size fractions, as measured using water or octanol, is $2.34 \pm 0.06 \mathrm{~g} / \mathrm{cm}^{3}$. This is the same as the density of the HIP CWF $\left(2.35 \pm 0.03 \mathrm{~g} / \mathrm{cm}^{3}\right)$, within experimental uncertainty. The densities of reference waste glass and the HIP CWF were measured as a check of the procedure. The measured densities are included in Table 2.

The bulk densities of the various PC CWF materials ranged from 1440 to $2170 \mathrm{~kg} / \mathrm{m}^{3}$. However, it was suspected that the bulk density, which was measured using large pieces, was not appropriate for crushed PC CWF material because most of the coarse porosity present in the bulk would be eliminated when the material was crushed to the $-100+200$ mesh size fraction. That is, fracturing of the PC CWF was expected to occur predominantly through pores, so that the pores would not be present within the resulting particles. In contrast, the bulk density is assumed to be appropriate for crushed HIP CWF because the porosity in that material is small enough that it remains in the crushed material. Therefore, we measured the densities of the crushed materials using the volume displacement method with either demineralized water or octanol. Different mesh-size fractions of crushed material were used to measure the density, depending on product availability. The densities of some materials were measured using several different size fractions. As suspected, the densities of the crushed PC CWF materials are higher than the bulk densities.

\subsubsection{Elemental Concentrations in CWF Materials}

The compositions of four samples of baseline PC CWF, six samples of advanced PC CWF, and two samples of HIP CWF were determined analytically by acidic dissolution of the solids following well-established methods [6] and analysis of the resulting solutions by ICP-AES. The compositions were also calculated from the compositions of the salt, zeolite, and glass based on their mass ratios in the final products. The results are summarized in Table 3. The concentrations of Cs and $\mathrm{K}$ are of particular interest because $\mathrm{CsCl}$ and $\mathrm{KCl}$ are volatile at the temperatures used to process the PC CWFs. These results show that the difference between the measured and calculated concentrations are the same within analytical uncertainty, which is assumed to be about $15 \%$ for both $\mathrm{Cs}$ and $\mathrm{K}$. Note that the measured concentrations of Cs are actually higher than the calculated concentrations. These results indicate that the amount of Cs that is volatilized, if any, is insignificant with respect to the total amount of $\mathrm{Cs}$ in the waste form. The elemental concentrations calculated from the amounts of 
salt-loaded zeolite and glass binder were used as the $f(i)$ in the normalized mass loss calculation rather than the measured concentrations, because they were assumed to be more reliable. This is because three separate digestions were required to obtain a complete composition analysis of the CWF, whereas only one digestion was required to determine the composition of each material. The gross compositions of the 50-mg samples of each material were homogeneous.

Different sieve fractions of crushed materials were analyzed to determine if fractionation of one or more phases occurred as a result of the crushing and sieving operations. For the baseline PC CWF, crushed materials of the $-100+200$ and the $-200+325$ mesh-size fractions were analyzed. For two advanced PC CWF materials, crushed materials of the $-100+200$, and $-200+325$, mesh-size fractions and material smaller than 325 mesh were analyzed, as well as material that was crushed but not sieved. The digestates were analyzed for dissolved cations with ICP-MS or ICP-AES, and with ion chromatography for dissolved anions. The measured concentrations of the major components are listed in Table 4. These results indicate that phases are not fractionated by crushing and sieving.

\subsubsection{Phase Identification}

Crushed samples of the baseline (25\% glass loading) and advanced PC CWF were analyzed with powder XRD and compared with XRD results for samples of HIP CWF that had been analyzed previously and with reference standards. The results are presented in Table 5 as measured d-spacings and relative intensities (for peaks with intensities greater than 1\%) for the PC CWF and HIP CWF spectra. The d-spacings for sodalite, nepheline, and halite reference materials from the International Centre for Diffraction Data files [7] are shown in Table 6. An internal standard was not used in the XRD analysis, and small offsets in d-spacings can be seen between the spectra for different materials. This is best seen by comparing the d-spacings for the most intense peaks for sodalite, near $3.624 \AA$. Because they dominate the spectra and are present in all samples, major sodalite peaks were used as an internal reference for identification of other peaks. In most cases, the shifts were small enough that the peak identifications were obvious. Most of the peaks in spectra of the PC CWF materials were assigned to sodalite. A small amount of nepheline was identified in the $25 \%$ glass loading PC CWF sample, based on the presence of weak peaks at 4.185 and $3.844 \AA$ in the XRD patterns. Nepheline was also identified in the HIP CWF, based on peaks between 4.165 and 4.173 $\AA$ and between 3.825 and $3.839 \AA$. The nepheline peaks were more intense in the HIP $\mathrm{CWF}$ spectra than in the PC CWF spectra. Halite $(\mathrm{NaCl})$ was identified in the day-today baseline PC CWF and advanced PC CWF spectra, based on peaks between 2.828 and $2.829 \AA$ and between 1.997 and $1.999 \AA$. Halite was also identified in HIP CWF spectra from peaks at $2.815 \AA$ and between 1.994 and $1.993 \AA$. The XRD results for the various CWF products are very similar. Analysis of the U,Pu-doped PC CWF with $\mathrm{XRD}$ showed the presence of $(\mathrm{U}, \mathrm{Pu}) \mathrm{O}_{2}$ in addition to sodalite, nepheline, and halite [2].

Albite was detected in developmental PC CWF samples heated at $850^{\circ} \mathrm{C}$ for 72 and $168 \mathrm{~h}$, based on peaks at 3.1231 and $3.1839 \AA$. Albite is an aluminosilicate mineral 
with an $\mathrm{Al} / \mathrm{Si}$ ratio of 1:3, which is lower than the 1:1 ratio in sodalite. The formation of albite in these samples is probably a result of interactions between the glass and sodalite phases. (The binder glass has an Al:Si ratio of about 1:7.5.) The developmental samples also contained small amounts of nepheline and halite. There was no evidence of albite in the waste forms processed for $24 \mathrm{~h}$ or less (based on the absence of peaks near 3.1 and $3.2 \AA$ in Table 5).

\subsubsection{Product Microstructure}

Polished cross sections of the PC CWF materials were examined with SEM. A photomicrograph of the $\mathrm{U}$ - and Pu-doped PC CWF is shown in Figure 2a. A photomicrograph of the $\mathrm{U}$ and Pu-doped HIP CWF is shown in Figure 2b, for comparison. The microstructures of both materials can be described as islands of sodalite domains that are about $100 \mu \mathrm{m}$ across distributed in a sea of glass binder. The intimate interfaces between sodalite and glass in both materials indicate that the glass fully wets the sodalite during processing. Note that the sodalite phase appears darker than the glass phase in the PC CWF, but lighter than the glass in the HIP CWF. This effect is probably due to small differences in how complete the glass flows between the granules of sodalite in the aggregate. The size of the typical sodalite domain $(100 \mu \mathrm{m})$ is similar to the size of the zeolite granule used in preparation of the CWF, and the size of the grains of sodalite within each domain (about $4 \mu \mathrm{m}$ ) is similar to the size of the polycrystalline grains of zeolite in the aggregate.

In Figs. $2 \mathrm{a}$ and $2 \mathrm{~b}$, the bright spots are about $1 \mu \mathrm{m}$ in cross section and are distributed as inclusions in the glass-phase clusters of mixed $\mathrm{U}$ and Pu oxide phases [2]. The presence of these colloid-sized inclusions is an important issue to be addressed in waste form qualification. One of the issues being addressed is whether these phases are retained at the altered surface or released to solution as colloids. Based on SEM examination of several samples, it appears that the $(\mathrm{U}, \mathrm{Pu}) \mathrm{O}_{2}$ inclusions are more randomly distributed in the glass phase of the PC CWF than in the HIP CWF, and they are concentrated at the sodalite/glass interfaces in the HIP CWF material. The regions shown in Figs. $2 \mathrm{a}$ and $2 \mathrm{~b}$ are consistent with this observation. Tests have shown the difference in the distribution of $(\mathrm{U}, \mathrm{Pu}) \mathrm{O}_{2}$ crystallites does not have a significant effect on their release behavior [2].

The pore structures are somewhat different in the PC and HIP CWF samples. In the HIP CWF, most pores are located in the glass near the sodalite/glass interface. In the PC CWF, small pores are distributed throughout the glass domain and the pores are not accumulated at the sodalite/glass interface. Only a small fraction of the pores are seen within the sodalite domains of either material. More pores are observed in the PC CWF than in the HIP CWF, which is consistent with the lower bulk density of PC CWF products compared to the HIP CWF. Neither nepheline nor halite was detected during SEM examination of any of the PC CWF materials. (Nepheline has not been observed in SEM examinations of the HIP CWF, either.) Halite was probably dissolved from the surface during sample preparation. Careful and water-free cutting and polishing are required to retain halite on the surface. Halite is routinely observed in optical 
microscopy and transmission electron microscopy samples in which the inclusions are not breached during preparation.

The SEM micrograph of the developmental PC CWF sample heated at $850^{\circ} \mathrm{C}$ for $168 \mathrm{~h}$ contained the features described above, as well as rectangular areas of slightly different contrast. EDS analyses showed these areas to be silicon-rich. Both the EDS results and the morphology of these features are consistent with albite, which was identified in the XRD spectra.

\subsection{CHEMICAL DURABILITY}

The following tests were conducted using samples from the 5-kg baseline PC CWF product: AFSM; RWS; 7-day and longer-term PCTs; and MCC-1 tests. The repeatability of the 7-day PCT with the PC CWF was measured with nine replicate tests. The AFSM, 7-day and longer-term PCTs, and the MCC-1 tests were used to determine if the dissolution behavior was the same for the PC CWF and HIP CWF materials.

\subsubsection{Accessible Free Salt Measurements (AFSMs)}

The AFSMs were conducted to evaluate the uniformity of the distribution of halite in a large PC CWF product. Samples of materials taken from the center, edge, and mid-radius of a 5-kg baseline product were subjected to AFSMs. The material from each section was crushed and sieved separately to isolate the $-100+200$ mesh size fraction for use in PCTs and the $-200+325$ mesh-size fraction for use in triplicate AFSMs. Only the chloride ion concentrations in the AFSM solutions were measured. The experimental data and results are given in Table 7. The chloride release was the same for samples from three different areas of the product. The $N L(\mathrm{Cl})$ was about 1 $\mathrm{g} / \mathrm{m}^{2}$ in AFSMs of the HIP CWF. This is about twice that measured for the PC CWF, and indicates that more halite had formed when the HIP CWF was processed than when the baseline PC CWF was processed. (Note that this does not mean there is twice as much halite in the bulk HIP CWF.)

\subsubsection{Rapid Water Soluble (RWS) Tests}

The RWS tests were conducted with the $-100+200$ mesh-size fraction of material taken from an edge section of the $5-\mathrm{kg}$ baseline product. To determine if there was an effect of sample size on the repeatability of the RWS, tests were conducted with $1,2,3$, and $4 \mathrm{~g}$ of PC CWF at a solid/water mass ratio at 1:10. The experimental data and results are given in Table 8. Examination of these results indicates that consistent results can be obtained with sample sizes of 2, 3, and $4 \mathrm{~g}$, but not with a sample size of 1 g. Although the mean of the three tests conducted with $1 \mathrm{~g}$ of CWF agrees well with the results of RWS conducted with more material, the responses in the three replicate tests with $1 \mathrm{~g}$ are significantly different. This may indicate that the distribution of halite in the waste form is not uniform on a $1-\mathrm{g}$ scale. About twice as much surface area is exposed in $1 \mathrm{~g}$ of $-200+325$ mesh material as in $1 \mathrm{~g}$ of $-100+200$ mesh material. This is apparently enough of a difference that a 1-g sample gives repeatable AFSM results but 
not repeatable RWS results. This is acceptable, since RWS will be conducted routinely with samples of more than $3 \mathrm{~g}$ of PC CWF for use in triplicate PCTs.

We also evaluated the effect on the RWS response of how the ethanol was added. That is, rather than adding the ethanol in a single wash step, we added several smaller aliquots of ethanol in multiple washes of the entire sample of CWF. The ethanol was decanted before subsequent aliquots were added. The use of multiple ethanol washes resulted in a $2 \mathrm{x}$ decrease in the amount of $\mathrm{Cl}$ dissolved in the RWS solution compared with a single wash. As shown in Table $8, N L(C l)$ was $0.35 \mathrm{~g} / \mathrm{m}^{2}$ for material taken from near the edge of the sample that was washed with three aliquots of absolute ethanol. The decrease in the RWS response with increasing ethanol washes is probably due to more efficient removal of halite-containing fines with multiple washes. The use of a single ethanol wash in many of the RWS tests discussed in this report provides an upper bound to the amount of halite in the CWF materials.

The crushed material of the $-100+200$ size fraction prepared from material taken from three different sections of the $5-\mathrm{kg}$ baseline product (center, edge, and mid-radius) was consolidated for use in the PCTs that are discussed in this report. The consolidated material was subjected to seven ethanol washes to remove fines prior to conducting the RWS procedure. The RWS response was $0.31 \mathrm{~g} / \mathrm{m}^{2}$ for this consolidated mixture, which is significantly lower than the values measured in RWS tests conducted with smaller amounts of materials subjected to a single ethanol wash.

The multiple ethanol washes were more efficient at removing fines (and the halite associated with those fines) than the single ethanol wash. The very large volume of ethanol used to wash the $38 \mathrm{~g}$ of consolidated material probably increased the washing efficiency because there was less settling prior to decanting, in addition to the effect of multiple washes. We suspect that more halite dissolved in the ethanol wash solutions during the multiple washes than during the single wash, although those wash solutions were not analyzed. Whereas a single ethanol wash will be used for product consistency tests that will be conducted with actual CWF in a hot cell, preparation of CWF for most laboratory tests was done using multiple washes to ensure that fines were removed when the objective of the test was to measure a property of the CWF for which the surface area must be estimated or when tracking the halite content was not necessary. Differences in the sample preparation steps must be taken into account when comparing test results with regard to halite behavior.

\subsubsection{Comparison of MCC-1 Test Results for PC and HIP CWF}

Duplicate 1-day and 3-day MCC-1 tests were run at $90^{\circ} \mathrm{C}$ to compare the dissolution rate of the PC CWF with that measured previously for the HIP CWF under the same test conditions. These short-term tests highlight differences in the dissolution of the sodalite phases in the two waste forms. Another set of MCC-1 tests was conducted at $125^{\circ} \mathrm{C}$ for 28 and 88 days with discs cut from the 5-kg baseline PC CWF (made with 50\% glass and 50\% salt-loaded zeolite) and with discs cut from a 50-g HIP CWF product made with $25 \%$ glass and $75 \%$ salt-loaded zeolite. These tests were run to 
compare test responses at greater extents of reaction, when the test response becomes dominated by the dissolution of binder glass. More CWF can dissolve before solution feedback effects lower the dissolution rate under the MCC-1 test conditions than under PCT conditions. This results in greater alteration of the PC CWF surface than occurs in the PCT, in which solution feedback effects slow the dissolution rate almost immediately. Therefore, the MCC-1 tests provide information regarding alteration of the sample surface during corrosion.

The $N L(i)$ for key components are given in Table 9 for the 1- and 3-day tests at $90^{\circ} \mathrm{C}$ and for the $28-$ and 88 -day tests at $125^{\circ} \mathrm{C}$. The dilute solution conditions that are maintained in short-term MCC-1 tests highlight the dissolution behavior of the waste form rather than the feedback effects of the test solution. Consideration of the test results in Table 9 provides some insight into the relative dissolution rates of the sodalite and glass binder phases in the PC CWF and HIP CWF, as well as the relative dissolution rates of the PC CWF and HIP CWF. In general, the values of $N L(\mathrm{~B}), N L(\mathrm{Li}), N L(\mathrm{Cl})$, and $N L(\mathrm{Si})$ in tests with $\mathrm{PC} C W F$ are similar to the corresponding values in tests with the HIP CWF. The values of $N L(\mathrm{Al})$ are significantly higher in tests with the PC CWF than with the HIP CWF at both temperatures and for all test durations.

The $\mathrm{B} / \mathrm{Si}$ ratio gives insight into the relative amounts of sodalite and binder glass that dissolved during the test. Since B is present only in the glass and Si is present in both sodalite and glass, the observation that $N L(\mathrm{~B})<N L(\mathrm{Si})$ for the 1-, 3-, and 28-day tests indicates preferential dissolution of sodalite. However, the observation that $N L(\mathrm{~B})$ $\geq N L(\mathrm{Si})$ in the 88 -day tests indicates that the binder glass is dissolving preferentially. For dissolution of borosilicate glasses, the values of $N L(\mathrm{~B})$ are almost always greater than the values of $N L(\mathrm{Si})$ because $\mathrm{B}-\mathrm{O}$ bonds are more easily hydrolyzed in the glass than $\mathrm{Si}-\mathrm{O}$ bonds. The values of $N L(\mathrm{Al})$ and $N L(\mathrm{Si})$ provide additional insight into the relative amounts of sodalite and glass that have dissolved, because the $\mathrm{Al} / \mathrm{Si}$ ratio is much higher in the sodalite phase (about 1:1) than in the glass binder (about 1:7.5). A test response where $N L(\mathrm{Al})>N L(\mathrm{Si})$ indicates preferential dissolution of sodalite. The results that the $N L(\mathrm{Al}) / N L(\mathrm{Si})$ ratio is higher in tests with the PC CWF than with the HIP CWF suggests that a greater fraction of sodalite is dissolved in tests with the PC CWF than with the HIP CWF under these test conditions.

Finally, the values of $N L(\mathrm{Cs})$ are significantly lower in tests with the PC CWF than with the HIP CWF. We interpret this to indicate that more Cs is incorporated into the glass binder than into sodalite in PC CWF materials than in HIP CWF materials. This could be due to the higher temperature and long processing time used to make the PC CWF.

Examination of the reacted materials with SEM indicates that the reacted surfaces of the HIP CWF and PC CWF were similar after reaction for 88 days at $125^{\circ} \mathrm{C}$. Representative photomicrographs of the surfaces of reacted PC CWF and HIP CWF are shown in Figs. $4 \mathrm{a}$ and $4 \mathrm{~b}$. Preferential dissolution occurs at the sodalite/glass domain boundaries in tests with both the PC CWF and HIP CWF, although the preference is slight. Note the greater pitting of the sodalite domains compared to the glass for both 
the PC CWF and HIP CWF. The similarity in surface alteration suggests that the chemical durability of the PC CWF is not affected by its greater porosity.

\subsection{SEVEN-DAY PRODUCT CONSISTENCY TESTS WITH VARIOUS PC CWF MATERIALS}

\subsubsection{Baseline PC CWF}

Three sets of triplicate 7-day PCTs were conducted at $90^{\circ} \mathrm{C}$ with the consolidated crushed material from the baseline PC CWF, to measure the repeatability of the PCT (i.e., the intra-laboratory precision). A single RWS test was applied to the mixture used in the test series: $N L(\mathrm{Cl})=0.31 \mathrm{~g} / \mathrm{m}^{2}$ (see Table 8). Since the RWS and PCT were conducted at a glass/water ratio of 1:10, the values of $N L(i)$ calculated for each part can be added directly to determine the total PCT response. The results for each set of triplicate tests and the mean values and standard deviation of the total $N L(i)$ (i.e., the sum of the masses released in the RWS and PCT steps) are summarized in Table 10. These values provided a measure of the intra-laboratory precision with which the PCT can be conducted with the PC CWF in our laboratory - the precision for each set of triplicate tests gives the precision with which the PCT can be conducted with the CWF; the overall precision of the three sets gives the precision with which the solutions can be analyzed. By comparing the standard deviations of each set of tests with the overall standard deviation of the nine tests, we conclude that the greatest contributor to the uncertainty in the test is the uncertainty for solution analysis. The intra-laboratory precision was expressed as the percent relative standard deviation: \%RSD = 100 - mean/standard deviation. The overall mean and standard deviation for the 9 tests were used to calculate the \%RSD to account for uncertainties in both running the tests and analyzing the solutions. The \%RSD values are included in Table 10.

The mean values of the total $N L(i)$ and the standard deviation for the three sets of triplicate tests are plotted in Figure 5. The results for different elements reflect the rates at which they are released from the CWF as it dissolves: $\mathrm{B}, \mathrm{Cl}, \mathrm{I}$, and Li give relatively high $N L(i)$ values and are released relatively rapidly, whereas $\mathrm{Al}, \mathrm{Cs}$, and $\mathrm{Si}$ give relatively low $N L(i)$ values and are released relatively slowly. The high values of $N L(\mathrm{Cl}), N L(\mathrm{Na})$, and $N L(\mathrm{I})$ are due to the rapid dissolution of halite in the RWS step. The values of $N L(\mathrm{Na})$ and $N L(\mathrm{Cl})$ are not equal because the mass fraction (and the mole fraction) of $\mathrm{Na}$ is higher than that of $\mathrm{Cl}$ in the $\mathrm{CWF}$, i.e., $f(\mathrm{Na})>f(\mathrm{Cl})$. Although equal moles of $\mathrm{Na}$ and $\mathrm{Cl}$ are released as the halite dissolves, division by a larger mass fraction value results in the value of $N L(\mathrm{Na})$ being lower than the value of $N L(\mathrm{Cl})$. The values of $N L(\mathrm{~B})$ and $N L(\mathrm{Li})$ are high because these elements are leached from the binder glass preferentially to $\mathrm{Al}$ and $\mathrm{Si}$ (and Cs) in the PCT step.

\subsubsection{Day-to-Day (DTD) Baseline PC CWF}

Seven-day PCTs were conducted with baseline PC CWF products (made with 50 mass $\%$ glass) that were made on 10 different days using the same source materials and following the same procedure. The objective was to measure the consistency with 
which laboratory-scale PC CWF products could be made to determine if uncertainty due to preparation of the test materials needs to be taken into account when comparing the PCT responses of different products. The precision for making laboratory-scale products is presumed to represent the precision for making full-size PC CWFs in a hot cell. Both the RWS and PCT fractions of the tests were evaluated. The results are summarized in Table 11 as the contributions of the RWS and PCT fractions, as well as their sum. Tests with samples DTD-1 and DTD-2 were run and analyzed in parallel as one set, and tests with the remaining eight samples were run and analyzed in parallel as another set. These sets are distinguished in this analysis because of possible differences in the analytical uncertainties of samples analyzed on different days.

The contributions of the RWS and PCT fractions to $N L(i)$ are plotted in Figs. 6a through $6 \mathrm{~h}$. It can be seen that the values of $N L(\mathrm{Cl})$ and $N L(\mathrm{I})$ are dominated by the contributions of the RWS solution, while $N L(\mathrm{Al}), N L(\mathrm{~B}), N L(\mathrm{Li})$, and $N L(\mathrm{Si})$ are dominated by the contributions of the PCT solution. The $N L(\mathrm{Cs})$ in the RWS is about half that in the PCT. The RWS and PCT contributions to $N L(\mathrm{Na})$ are about the same. The average mole ratios of $\mathrm{Na}$ and $\mathrm{Cl}$ in the RWS fraction solutions for the 10 tests are $1: 1$, which is the $\mathrm{Na} / \mathrm{Cl}$ mole ratio in halite. The observation of equimolar amounts of $\mathrm{Na}$ and $\mathrm{Cl}$ in the RWS solution is consistent with the RWS solution chemistry being dominated by the dissolution of halite. The concentrations of Li, K, Cs, and I in the RWS solution are low, which indicates that these elements are present in the halite inclusions at low concentrations (about $1 \% \mathrm{~K}$ and $2 \% \mathrm{Li}$ and trace levels of $\mathrm{Cs}$ and $\mathrm{I}$ ). In contrast, the average mole ratio of $\mathrm{Na}$ and $\mathrm{Cl}$ in the PCT fraction solutions is about 4.5:1; this is higher than the gross $\mathrm{Na} / \mathrm{Cl}$ mole ratio of the $\mathrm{CWF}$, which is about 3.6:1. Although the binder glass used to make the CWF does not contain $\mathrm{Cl}$, up to about 1 mass \% $\mathrm{Cl}$ may dissolve in the glass during processing. This would give a maximum $\mathrm{Na} / \mathrm{Cl}$ ratio of about 5.7:1 in the glass. The measured $\mathrm{Na} / \mathrm{Cl}$ ratio in the PCT solutions suggests that similar amounts of sodalite and glass have dissolved.

The means of the total $N L(i)$ values (i.e., the sum of the RWS and PCT parts) for the DTD samples are plotted in Figure 7. Examination of these data indicates that sample production (and testing) is repeatable, with two exceptions - the RWS fraction of DTD-9 and the PCT fraction of DTD-2. The values of $N L(\mathrm{Al}), N L(\mathrm{Cs})$, and $N L(\mathrm{Si})$ for the RWS fraction of DTD-9 are more than two standard deviations higher than the mean values of $N L(i)$ (see Table 11). The value of $N L(\mathrm{Na})$ is also noticeably high, but is within two standard deviations of the mean value of $N L(\mathrm{Na})$. We suspect that the RWS solution for sample DTD-9 was contaminated with fine particulates of PC CWF that had not been removed by the $450-\mathrm{nm}$ filtration step of the RWS procedure. This is suspected because $\mathrm{Al}$ and $\mathrm{Si}$ are not present in halite and are instead released primarily during dissolution of the glass and sodalite. Very little glass or sodalite dissolves during the RWS step. In this series of RWS tests, the $N L(i)$ for $\mathrm{Cl}$, I, and $\mathrm{Na}$ are consistent for the 10 products (within two standard deviations), which indicates that the same amount of halite had dissolved in all 10 tests. The deviation of the RWS test from the mean with sample DTD-9 is probably due to inefficient filtration rather than a variance in the product. DTD-9 test results were excluded from the calculations of the mean, standard deviation, and the relative standard deviation reported in Table 11. 
The $N L(\mathrm{~B})$ for the PCT fraction of test DTD-2 is lower than the mean, and the $N L(\mathrm{I})$ value is higher than the mean. It is likely that the iodide content was a little higher in that sample than in the others, due to a nonuniform distribution of iodide in the waste form. Components present in low concentrations are not expected to be as uniformly distributed in the CWF as components present in higher concentrations. This may also be the reason for the low value of $N L(\mathrm{~B})$. The values of $N L(\mathrm{Na})$ and $N L(\mathrm{Si})$ for the PCT fraction of DTD-2 are consistent with the values in other tests. Therefore, the DTD-2 test results were included in the calculations of the mean and standard deviation.

Initially, the precision measured in the nine replicate tests with the baseline PC CWF presented in Section 4.3.2 was used to evaluate the repeatability of the DTD materials. However, the precision of those replicate tests was much better than that measured for the PCT part in an inter-laboratory study (ILS) conducted with advanced PC CWF (see Section 4.4.2). The intra-laboratory precision values measured in the ILS, expressed as \%RSD for $\mathrm{Al}, \mathrm{B}, \mathrm{Na}$, and $\mathrm{Si}$, are $\mathrm{Al}=4.1 \%, \mathrm{~B}=5.0 \%, \mathrm{Na}=10 \%$, and $\mathrm{Si}=$ $2.9 \%$. The results of replicate tests within the same laboratory are expected to agree with these values. The inter-laboratory precision values as $\% \mathrm{RSD}$ are $\mathrm{Al}=5.6 \%, \mathrm{~B}=$ $15 \%, \mathrm{Na}=11 \%$, and $\mathrm{Si}=9.1 \%$. The results of replicate tests conducted in different laboratories are expected to agree with these values. The precision values measured in the ILS provide a more representative measure of test precision with which to evaluate the repeatability of making the PC CWF and composition effects than the values in Table 10. By using \%RSD, the precision of the measured concentrations and the precision in the calculated $N L(i)$ values can be compared directly. Comparing the $\%$ RSD values in Table 11 with those from the ILS, the $N L(\mathrm{Na})$ and $N L(\mathrm{Si})$ responses for the 9 DTD products (excluding DTD-9) agree within the \%RSD for both the intralaboratory and inter-laboratory precision, the $N L(\mathrm{~B})$ response agrees within the interlaboratory precision, and the $N L(\mathrm{Al})$ response exceeds both the expected intra-laboratory and inter-laboratory precision values.

\subsubsection{Glass Loading PC CWF}

Several products were made with lower fractions of binder glass than used in the baseline PC CWF (i.e., less than 50\%) to study the effects of increased the waste loadings. Products that we refer to as glass loading PC CWF were made with 25, 30, 35, 40 , or $45 \%$ glass (by mass) to measure the effect of the glass/sodalite ratio on the microstructure, density, and chemical durability of the CWF material. These samples were made using the same heating profile that was used for the baseline PC CWF, namely, a hold temperature of $850^{\circ} \mathrm{C}$ and a hold time of $4 \mathrm{~h}$. Products made with the low glass contents tended to be more friable and had lower bulk densities than products made with higher glass contents. All of the crushed material with a particular glass loading PC CWF was prepared in a single batch, and one RWS procedure was run for the entire batch. The washed materials were then used in triplicate PCTs.

The results of PCTs conducted with the glass loading PC CWF are summarized

in Table 12. The contributions of the RWS and the means of the contributions from the PCT fractions in replicate tests with each material are plotted in Figs. 8a through 8h. 
Note that we have plotted the concentrations rather than the $N L(i)$ to determine if the concentrations of elements provide a measure of the sensitivity of the PCT to changes in the gross composition of the waste form. The concentrations of $\mathrm{Al}$ and $\mathrm{Si}$ are relatively insensitive to the amounts of glass and salt-loaded zeolite that are used to make the CWF because these elements are present in both the glass and the zeolite. Boron is present only in the glass and provides a valuable measure of the relative amount of glass in the CWF. The boron concentration clearly increases with the glass content (see Figure $8 \mathrm{~b}$ ). Although Cs, Cl, Li, and I are present only in the SLZ, their concentrations in the test solutions actually increase as the amount of SLZ decreases and the amount of glass increases (the Cl PCT fraction increases, but the Cl RWS fraction decreases as the amount of glass increases). The observation that the greatest differences occur in the RWS fractions of $\mathrm{Na}, \mathrm{Cl}$, and I probably indicates that different amounts of halite form in PC CWFs made with different glass loadings. There is about twice as much $\mathrm{Na}$ in the SLZ as in the glass, so, like $\mathrm{Cl}$ and $\mathrm{I}$, the release of $\mathrm{Na}$ was expected to decrease as the glass content was increased. However, Figure $8 \mathrm{~g}$ shows that the release of $\mathrm{Na}$ in the RWS step increases with the glass content and the release of $\mathrm{Na}$ in the PCT step is nearly constant. The Cs concentration clearly decreases in the RWS and PCT steps as the amount of SLZ decreases, but the Li concentrations are insensitive to the relative amounts of SLZ and glass. This is consistent with the hypothesis that Li dissolves into the glass during processing.

The mean concentrations of $\mathrm{B}, \mathrm{Cl}, \mathrm{Na}$, and $\mathrm{Si}$ in triplicate tests with each of the glass loading PC CWF materials are plotted in Figure 9 with uncertainty bars drawn at two standard deviations. The results are plotted on a log scale to better distinguish the responses for the different materials. This plot shows the sensitivity of the test response to the glass content and gives insight into the sensitivity of the 7-day PCT to the glass/zeolite mass ratio. The different glass loading products are distinguishable at the 95\% confidence level if the PCT responses differ by more than two standard deviations. Only the release of $\mathrm{B}$ can be used to distinguish the glass loading in the PC CWF (fabricated at $850^{\circ} \mathrm{C}$ ), as shown in Figure 9. This is expected, since B is present only in the glass binder and other components are present in both the glass and sodalite phases. While these results confirm that boron can be used to monitor the glass content of CWF materials, tests are needed with $\mathrm{PC}$ CWFs made at $915^{\circ} \mathrm{C}$ (which is the current processing temperature) with glass contents near $25 \%$ to determine the precision and accuracy to which the glass content can be tracked using the PCT response.

\subsubsection{Tests with the Advanced PC CWF}

Advanced PC CWF products were made with 75 mass\% SLZ and 25 mass\% glass at higher temperatures $\left(915^{\circ} \mathrm{C}\right)$ and longer hold times $(16 \mathrm{~h}$ and $24 \mathrm{~h})$ than the baseline PC CWF, in an attempt to increase the bulk density of the PC CWF without adversely affecting chemical durability. Products made for testing varied in size from $20 \mathrm{~g}$ to $26 \mathrm{~kg}$. Samples from all of the advanced PC CWF products were subjected to RWS tests to measure halite abundance. The experimental data and results for the RWS tests are given in Table 13. The mean $N L(\mathrm{Cl})$ of the five RWS tests with materials from the 20 -g products was the same as the mean $N L(\mathrm{Cl})$ for the larger products, at the $95 \%$ 
confidence level, indicating that neither the product size nor the heating time $(16 \mathrm{~h}$ versus $24 \mathrm{~h}$ ) had a significant effect on the amount of halite that was generated.

Triplicate 7-day PCTs were conducted with material from advanced PC CWF products PC02606 (a 20-g product) and PC02701 (a 26-kg product). Samples were taken from two different locations in PC02701 (designated as PC02701-2 and PC0270113). The results for these tests are given in Table 14. The values of $N L(i)$ calculated from the concentrations measured in the RWS and PCT solutions are plotted in Figs. $10 \mathrm{a}-10 \mathrm{~h}$. As seen in tests with other PC CWF products, the total values of $N L(\mathrm{Cl})$, $N L(\mathrm{I})$, and $N L(\mathrm{Na})$, i.e., the sum of the RWS and PCT parts, are dominated by the contributions of the RWS solutions, while values of $N L(\mathrm{Al}), N L(\mathrm{~B}), N L(\mathrm{Li})$, and $N L(\mathrm{Si})$ are dominated by the contributions of the PCT solutions. This difference occurs because the chemistries of the RWS solutions are determined by the dissolution of halite, while those of the PCT solutions are dominated by the dissolution of the glass and sodalite phases. Note that the greater releases of Li occur in the PCT whereas the greater release of $\mathrm{Na}$ occurs in the RWS. This indicates that $\mathrm{Li}$ is more efficiently retained in the sodalite structure and/or dissolves into the glass to a greater extent than $\mathrm{Na}$. The boron concentration decreased almost twofold as the product size increased from $20 \mathrm{~g}$ to $26 \mathrm{~kg}$. The reason for the decrease is not clear. It is not expected that product size alone is responsible for the difference in $\mathrm{B}$ retention. It is possible that the difference is a result of the greater temperature gradients expected in the processing of larger products. The effects of processing temperature on the PCT response are discussed in Section 4.6.

Most of the Al and Si detected in the RWS solutions can be attributed to the presence of fines. Evidence for this is given in Table 15, which shows the concentrations measured in aliquots of three RWS solutions that were first passed through a $450-\mathrm{nm}$ pore-size filter and then through a $20-\mathrm{nm}$ pore-size filter. The ratios of the concentrations measured in the $450-\mathrm{nm}$ filtrate to those measured in the $20-\mathrm{nm}$ filtrates are included in the table to facilitate the comparison. The ratios for $\mathrm{B}, \mathrm{Cs}, \mathrm{K}, \mathrm{Li}$, $\mathrm{I}$, and $\mathrm{Na}$ are essentially 1, which indicates that they are dissolved in the test solutions. The ratios for $\mathrm{Al}$ and $\mathrm{Si}$ are significantly greater than 1, which means that they are present mostly as particulates suspended in the test solutions. The total $N L(i)$ (the sum of the $N L(i)$ values for the RWS and PCT fractions) are summarized in Figure 11. Replicate tests with each material show good test repeatability, and the differences between the results of tests with samples from the different products are attributed to analytical uncertainty in the solution analyses.

\subsubsection{Tests with Developmental PC CWF}

The developmental PC CWFs were prepared to study the interactions between the salt-loaded zeolite (or sodalite) and the glass binder during processing. These products were rapidly heated at $850^{\circ} \mathrm{C}$ and held at that temperature for 4,72 , and $168 \mathrm{~h}$. Triplicate RWS tests were conducted with samples of the developmental PC CWF to compare the relative amounts of halite that were generated during the different hold times. The RWS data and results are given in Table 16. The $N L(\mathrm{Cl})$ increases with heating time and ranges from 0.054 to $6.69 \mathrm{~g} / \mathrm{m}^{2}$ at the 4 and $168 \mathrm{~h}$ hold times, 
respectively. The XRD results are summarized in Table 17. These show that the intensities of the halite peaks are greater for CWF made with a longer heating time. This is consistent with the greater chloride losses that occurred in the RWS tests with those materials. The XRD and SEM results also showed that albite formed in the CWF held at $850^{\circ} \mathrm{C}$ for 72 and $168 \mathrm{~h}$. These data indicate that interactions do occur between the sodalite and the glass that result in the formation of halite. Analysis of the microstructure with SEM indicated that the sizes of the sodalite domains decrease with an increase in the heating time. This suggests that the zeolite rapidly converts to sodalite, then the sodalite slowly dissolves into the glass. The composition of the glass at the glass/sodalite interface changes as sodalite dissolves. Halite inclusions form as the capacity of glass for $\mathrm{Cl}$ is reached, and albite forms when its solubility limit in the glass is reached (perhaps as the waste form cools). The extent of sodalite dissolution and formation of halite and albite all increase with longer process hold time (and probably temperature).

\subsection{COMPARISON OF SEVEN-DAY PCT RESULTS}

\subsubsection{Results for Different Products}

The 7-day PCT was used to compare the chemical durabilities of various CWF products. The releases of specific elements are used to track the dissolution of the halite, sodalite, and glass binder phases in the CWF. Figure 12 and Table 18 summarize the means of the total $N L(i)$ values for several elements from replicate 7-day tests with the 5-kg and day-to-day baseline PC CWFs, glass loading PC CWF with 25 mass\% glass, advanced PC CWF, and HIP CWF. Also included in Table 18 are the results of 7-day PCTs with HIP CWF and PC CWF made with Pu-doped salts [2]. Because calculation of $N L(i)$ takes into account the differences in the CWF compositions, the $N L(i)$ for tests with different CWF materials can be compared directly. The comparison of these results allow features that are common to the PC and HIP CWF products to be identified and determination of whether there are significant differences in the durability of the different materials.

Figure 12 shows the greatest difference is in the $N L(\mathrm{Cl}), N L(\mathrm{I})$, and $N L(\mathrm{Na})$ values for the advanced PC CWF compared with the other CWF materials. More halite was formed in the advanced PC CWF and Pu-doped PC CWF than in the other PC CWF products. The $N L(\mathrm{Cl})$ in the RWS of the advanced PC CWF represented over $90 \%$ of the overall $N L(\mathrm{Cl})$ for that material. In contrast, the $N L(\mathrm{Cl})$ in the RWS represented about $70 \%$ of the overall $N L(\mathrm{Cl})$ of the $25 \%$ glass loading PC CWF and about $80 \%$ of the overall $N L(\mathrm{Cl})$ for the day-to-day baseline PC CWF. The difference in halite abundance may result from the higher processing temperature and the longer hold time used to make the advanced PC CWF and Pu-doped PC CWF $\left(915^{\circ} \mathrm{C}\right.$ and $875^{\circ} \mathrm{C}$, respectively, for $24 \mathrm{~h}$ ) compared to the glass loading and baseline products $\left(850^{\circ} \mathrm{C}\right.$ for $4 \mathrm{~h}$ ). The effects of processing temperature and time are discussed in Section 4.6.

Also seen in Figure 12 are significant differences in $N L(\mathrm{~B})$ for the different CWFs. The release of boron is important because it provides a unique measure of glass 
dissolution. In the 7-day PCTs, the $N L(\mathrm{~B})$ results in tests with the advanced PC CWF and $\mathrm{Pu}$-doped $\mathrm{PC} \mathrm{CWF}$, which were made at $915^{\circ} \mathrm{C}$ and $875^{\circ} \mathrm{C}$, respectively, are 2 to 4 times lower than in tests with the $25 \%$ glass loading PC CWF and 10 times lower than in tests with the $5-\mathrm{kg}$ and the DTD-baseline products, all of which were made at $850^{\circ} \mathrm{C}$. This suggests that the glass binder phase is more durable when the PC CWF is fabricated at higher temperatures. This may be due to subtle changes in the glass composition due to dissolution of the sodalite in the glass.

\subsubsection{Comparison of PCT Results for Different Laboratories}

An inter-laboratory study was conducted using advanced PC CWF materials to measure the precision with which the PCT could be conducted with a CWF. A detailed discussion of the inter-laboratory study with CWF has been published elsewhere [8]. Participants conducted one RWS test and three PCTs with the PC CWF for 7 days at $90^{\circ} \mathrm{C}$. The PC CWF used by all participants was crushed, sieved, and washed with ethanol at ANL to eliminate variances in these steps from the study. Six independent data sets were generated during the study, where a data set is considered independent if a different experimentalist conducted the test and a unique instrument was used to analyze the test solutions. Three sets of data were generated at ANL-East, and one data set was generated at ANL-West, Savannah River Technology Center, and Pacific Northwest National Laboratory. The test results are summarized in Table 19. They were used to calculate precision statistics following the methods recommended in ASTM standard E691 [9]. The means of the $\mathrm{Al}, \mathrm{B}, \mathrm{Na}$, and $\mathrm{Si}$ concentrations measured by each participant in triplicate tests are plotted in Figure 13. The overall mean concentrations are shown by the horizontal lines for $\mathrm{Al}=24.3, \mathrm{~B}=2.25, \mathrm{Na}=30.2$, and $\mathrm{Si}=32.6 \mathrm{mg} / \mathrm{L}$. Note that the $\mathrm{Al}$ results of participant $\mathrm{G}$ were excluded from the precision statistics as an outlier. The precision statistics for the inter-laboratory study with the advanced PC CWF are summarized in Table 20. The ASTM standard E691 provides a method for quantifying the intra-laboratory statistics using the $k$-statistic. The $k$-statistic is calculated as the ratio of the standard deviation for the replicate tests conducted by a participant to the pooled standard deviation for all participants. This gives a measure of the repeatability for each participant. Values of the $k$-statistic are plotted in Figure 14. Critical values as given by ASTM standard E691 depend on the number of participants and the number of replicate tests. For 6 participants and triplicate tests, the critical value of the $k$-statistic is 1.98 . Values greater than 1.98 indicate that non-random sources of error probably exist. The Na value of Participant $\mathrm{G}$ exceeds the critical value. The Al value of Participant $\mathrm{G}$ also exceeds the critical value, but this was already excluded as an outlier. Values significantly less than the critical value indicate that a laboratory may be reporting results to too few significant figures. The ASTM standard E691 provides the $h$-statistic to quantify the inter-laboratory consistency. The $h$-statistic is calculated as the difference between the mean value for a participant and the consensus mean divided by the standard deviation for the replicate tests conducted by that participant. Values of the $h$-statistic are plotted in Figure 15. The critical value for six participants is \pm 1.92 . The values for all participants are within the critical value. Therefore, all test results (except for the Al value of Participant G) were included in calculation of the interlaboratory test precision. 
The results of the ILS with the CWF are compared with the precision measured previously for conducting the PCT with borosilicate glasses in Table 21, which shows a comparison of the precision in terms of the standard deviation for intra-laboratory precision $\left(s_{r}\right)$ and the standard deviation for inter-laboratory precision $\left(s_{R}\right)$. The precision for conducting a PCT with CWF material is the same as that for conducting a PCT with a borosilicate glass.

\subsubsection{Comparison of $N L(i)$ from PC CWF, HIP CWF, and EA Glass}

We have used the 7-day PCT to compare the dissolution behaviors of PC CWF products made using various heating temperatures and durations. We have compared $N L(i)$ values for tests with various PC CWFs and between the HIP CWF and PC CWF. Among the PC CWF materials, the most significant differences in the dissolution behaviors were (1) the greater releases of $\mathrm{Na}$ and $\mathrm{Cl}$ in the RWS with the PC CWF prepared at high temperatures compared with those prepared at lower temperatures, which reflect the greater amounts of halite that are generated during processing at the higher temperatures, and (2) the smaller release of B that occurred in 7-day PCTs with PC CWFs prepared at high temperatures compared with those prepared at lower temperatures. However, these differences are minor when the responses in the PCT with either the PC CWF or HIP CWF are compared with the response of Environmental Assessment (EA) glass. The response of EA glass in the 7-day PCT is specified as a benchmark for high-level waste glasses in the WASRD [4] and will probably be used as a benchmark for measuring the consistency of the CWF. The results for replicate PCTs with the EA glass are $N L(\mathrm{~B})=8.5 \mathrm{~g} / \mathrm{m}^{2}, N L(\mathrm{Li})=4.8 \mathrm{~g} / \mathrm{m}^{2}, N L(\mathrm{Na})=6.7 \mathrm{~g} / \mathrm{m}^{2}, N L(\mathrm{Si})=$ $2.0 \mathrm{~g} / \mathrm{m}^{2}$ [12]. The corresponding values for these elements calculated from the concentrations measured in the inter-laboratory study with the advanced PC CWF are $0.055,0.47,0.89$, and $0.080 \mathrm{~g} / \mathrm{m}^{2}$, respectively. For all elements, the $N L(i)$ values in tests with the CWF are significantly lower than the $N L(i)$ values in tests with EA glass. The highest value for any of the CWF products tested is $N L(\mathrm{Cl})$, which is about $4 \mathrm{~g} / \mathrm{m}^{2}$. This is significantly lower than the highest value for the EA glass, which is $N L(\mathrm{~B})=8.5$ $\mathrm{g} / \mathrm{m}^{2}$. These results show that CWF can be produced to meet the WASRD requirement for the PCT.

\subsection{LONG-TERM PRODUCT CONSISTENCY TESTS}

PCTs are commonly conducted for time periods longer than 7 days (often for several years) to study the corrosion behavior of waste forms in solutions that become highly concentrated with dissolved waste form components. Such solutions could be generated in a disposal system after several thousand years. Although waste forms will not be crushed, the $S / V$ ratio used in PCTs is similar to the effective $S / V$ ratio for a thin layer of water on the surface of a monolithic waste form. The most important aspect is that the chemistry of the solution in contact with the glass in long-term PCTs is similar to that expected in a disposal system after small amounts of water contact the waste forms for long times. Long-term PCTs can also be used to measure the apparent 
solubility limit of silica with respect to glass, which is a parameter in the glass dissolution model. We conducted long-term tests with the baseline PC CWF and the advanced PC CWF to address both issues, and for comparison with the results of longterm PCTs conducted previously with the HIP CWF. We note that a slightly different sample preparation procedure was used for PCTs with the HIP CWF than was conducted previously. The crushed and sieved HIP CWF material was washed several times with absolute ethanol to remove fines, but was not washed with water prior to testing. Although a small amount of halite may have dissolved during the ethanol washes, most of the halite dissolved during the PCT step, probably immediately when the water was added. Therefore, the results of PCTs with the HIP CWF are compared with the sum of the RWS and PCT parts of PCTs conducted with the PC CWF.

Long-term PCTs were also conducted with as-received binder glass and with PC glass that had been remelted at $915^{\circ} \mathrm{C}$. These tests were conducted to compare the release behavior of boron from binder glass that is part of the CWF with the release behavior of boron from pure binder glass. This was done to determine empirically if the physical presence of sodalite and the inclusion phases (halite and oxides) affected the dissolution rate of the glass. These test results were also used to measure the apparent solubility limit of silica with respect to glass for comparison with the apparent solubility limit of silica with respect to the baseline PC CWF and the advanced PC CWF.

\subsubsection{Long-Term PCTs with Baseline PC CWF}

Long-term PCTs were conducted with the consolidated material from the 5-kg baseline product at $90^{\circ} \mathrm{C}$ for 28 days (duplicate tests), 56 days (duplicate tests), and 89 days (six tests). The test results are summarized in Table 22. The mean $N L(i)$ values for the PCT fractions for the four time periods are plotted in Figure 16. The contributions from the RWS test solution are not included in the plotted data because a single RWS test was conducted for all the material used in the PCT and the same RWS fraction concentrations are used for all test durations. The results for 7-day tests in Figure 16 differ from the results in Figure 5, primarily due to the dissolution of halite in the RWS step, which is included in Figure 5 but not in Figure 16. The solution chemistry of the PCT fraction is dominated by the dissolution of the glass binder, as indicated by the preferential release of boron relative to silicon. The results in Figure 16 show nearly equal releases of boron and lithium, sodium and chloride, and aluminum and silicon over time. We believe that boron and lithium are released as the glass binder dissolves, sodium and chloride are released (in the PCT fraction) primarily as the sodalite dissolves, and aluminum and silicon are released as both the glass binder and sodalite dissolve. As discussed previously, these test results strongly suggest that lithium becomes incorporated into the glass during processing, probably as part of an ion exchange reaction with other alkali metals in the glass. 
Most of the $\mathrm{Na}$ and $\mathrm{Cl}$ are released as sodalite dissolves, although small amounts of sodium and chloride are released in the PCT fraction as the glass binder dissolves. Inspection of Figure 16 indicates that the release of boron continues through (and likely beyond) 91 days, while little additional sodium, chloride, or silicon are released after about 28 days. This suggests that the glass binder will continue to dissolve whereas the sodalite will not. That is, the solution becomes saturated with respect to sodalite after about 28 days but remains undersaturated with respect to the glass for beyond 91 days under these test conditions. This has important implications on the modeling and longterm prediction of CWF dissolution behavior. Under repository-relevant conditions of limited solution volumes, CWF degradation will be dominated by dissolution of the binder glass and will not be affected significantly by dissolution of sodalite.

\subsubsection{Long-Term PCTs with Advanced PC CWF}

Long-term PCTs were conducted with advanced PC CWF materials that were prepared as $500-\mathrm{g}$ products at $915^{\circ} \mathrm{C}$ with a hold time of $16 \mathrm{~h}$. Enough material was crushed and sieved to obtain the amount of $-100+200$ mesh sieve fraction needed to conduct all tests. All of the CWF material was subjected to a single RWS test and then dried. An aliquot of the RWS solution was analyzed for chloride, and the remaining solution was acidified for analysis of other components with ICP-MS. Tests were conducted at $90^{\circ} \mathrm{C}$ in demineralized water using $\mathrm{CWF} /$ water mass ratios of $1: 10$ and 1:1. These gave $S / V$ ratios of about 2300 and $23,000 \mathrm{~m}^{-1}$, respectively. The test matrix is given in Table 23.

At test termination, aliquots of the PCT solution were analyzed for $\mathrm{pH}$ and chloride ion concentrations. The remaining solution was passed through a $450 \mathrm{~nm}$ poresize filter to remove any suspended material and then acidified with high-purity nitric acid and analyzed with ICP-MS. The chloride ion concentration was determined with a chloride ion selective electrode. The $\mathrm{pH}$ was measured with a combination electrode at room temperature after the solution had cooled.

The solution concentrations of key components in the PCT conducted with advanced PC CWF are given in Table 24. The values of $N L(i)$ that were calculated using these measured concentrations are given in Table 25. The values of $N L(\mathrm{~B})$, $N L(\mathrm{Cl}), N L(\mathrm{Na})$, and $N L(\mathrm{Si})$ are plotted vs. test duration in Figs. $17 \mathrm{a}-17 \mathrm{~d}$. The values of $N L(\mathrm{~B}), N L(\mathrm{Cl})$, and $N L(\mathrm{Na})$ for tests with advanced PC CWF and HIP CWF differ by about $10 \times$, but the values of $N L(\mathrm{Si})$ are similar in tests with the two materials. The values of $N L(\mathrm{Cl}), N L(\mathrm{Na})$, and $N L(\mathrm{Si})$ are nearly constant as a function of time, whereas the value of $N L(\mathrm{~B})$ increases through about 200 days. The values of $N L(\mathrm{Na})$ and $N L(\mathrm{Cl})$ are dominated by the dissolution of halite at the outer surface of the crushed CWF. Dissolution of halite occurs immediately when the CWF is placed in the water. The additional $\mathrm{Na}$ and $\mathrm{Cl}$ released as the glass and sodalite dissolve are negligible compared

to the $\mathrm{Na}$ and $\mathrm{Cl}$ released by halite dissolution. The increase in $N L(\mathrm{~B})$ indicates that the binder glass continues to dissolve. 


\subsubsection{Long-Term PCTs with Binder Glass}

Long-term PCTs were conducted with the as-received binder glass and with PC glass (binder glass that was remelted at $915^{\circ} \mathrm{C}$ ) to compare the dissolution of glass itself with the dissolution of the glass phase in the PC CWF. The test matrix is given in Table 26. The as-received binder glass was sieved to obtain the $-100+200$ mesh size fraction, which was then washed with absolute ethanol to remove fines. The PC glass was crushed and then sieved and washed. Neither glass was subjected to the RWS test because neither glass contained halite inclusions. Most PCTs were conducted at glass/water mass ratios of 1:1 or 1:10. The 182- and 364-day tests with the as-received binder glass were conducted at lower mass ratios because of the limited amount of sample that was available. The solution concentrations are given in Table 27, and the values of $N L(i)$ calculated from these concentrations are given in Table 28. The values of $N L(\mathrm{~B}), N L(\mathrm{Na})$, and $N L(\mathrm{Si})$ in tests with $\mathrm{PC}$ glass and binder glass are plotted against the test duration in Figure 18a for tests at $2300 \mathrm{~m}^{-1}$ and in Figure $18 \mathrm{~b}$ for tests at 23,000 $\mathrm{m}^{-1}$. At both $S / V$ ratios, the values of $N L(\mathrm{~B})$ and $N L(\mathrm{Na})$ are similar and significantly greater than the values of $N L(\mathrm{Si})$ at all test durations. Preferential release of $\mathrm{B}$ and $\mathrm{Na}$ is commonly observed in tests with borosilicate glasses. The release of Na occurs rapidly through an ion exchange mechanism until an alkali-depleted layer becomes a barrier to the diffusion of water into the glass. Boron is released rapidly by hydrolysis as water diffuses into the glass. Hydrolysis of silicon occurs at a lower rate than hydrolysis of boron, but becomes rate controlling after the alkali-depleted layer is formed. This is why the values of $N L(\mathrm{~B})$ and $N L(\mathrm{Na})$ "roll over" and become nearly constant after about 200 days.

\subsubsection{Apparent Solubility Limit of Orthosilicic Acid}

The long-term test results provide a measure of the silica solubility limit with respect to the CWF and binder glass materials. The CWF degradation model includes a term for the chemical affinity based on the ratio of the orthosilicic acid $\left(\mathrm{H}_{4} \mathrm{SiO}_{4}\right)$ concentration in the solution to its saturation concentration. These tests are used to determine the value of that parameter. The solubility of orthosilicic acid is constant with respect to $\mathrm{pH}$, but the amount of silica in solution will increase due to dissociation as the $\mathrm{pH}$ exceeds 9.66 at $30^{\circ} \mathrm{C}$. The $\mathrm{pH}$ that was measured at room temperature is expected to be slightly higher than the $\mathrm{pH}$ at $30^{\circ} \mathrm{C}$ due to the decrease in the dissociation constant of water with temperature and other temperature-dependent chemical effects. Thus, dissociation of orthosilicic acid is not expected to occur if the room temperature $\mathrm{pH}$ is less than about 9.7. The highest $\mathrm{pH}$ value measured were 9.52 for tests with the CWF and 8.65 for tests with binder glass, so all of the silicon is assumed to be present in solution as orthosilicic acid in all tests with CWF and binder glass. The orthosilicic acid concentrations were calculated by multiplying the measured Si concentrations by the molecular weight of $\mathrm{H}_{4} \mathrm{SiO}_{4}$ (96) and dividing by the atomic weight of $\mathrm{Si}$ (28); that is, $96 / 28=3.43$.

The concentrations of orthosilicic acid attained in tests with CWF and binder glass are summarized in Tables 29 and 30, respectively. These concentrations are 
plotted in Fig. 19a for tests with the advanced PC CWF and HIP CWF and in Figure 19b for tests with $\mathrm{PC}$ glass and binder glass. The $\mathrm{pH}$ values that were measured at room temperature are included in the tables. Tests conducted at $S / V$ ratios of 2300 and 23,000

$\mathrm{m}^{-1}$ are shown on the same graph using filled and open symbols, respectively. The solution concentrations are expected to approach a limiting value as the test duration increases, and tests conducted at high $S / V$ ratios are expected to become saturated more quickly than tests at low $S / V$ ratios. The $S / V$ ratio is expected to affect the concentrations that are attained after a particular test duration, but not the solubility limit.

We consider first the results for the binder glass. From Figure 19b, the apparent solubility limit for PC glass is higher than that for the as-received binder glass: about $420 \mathrm{mg} \mathrm{H}_{4} \mathrm{SiO}_{4} / \mathrm{L}$ for $\mathrm{PC}$ glass compared to about $360 \mathrm{mg} \mathrm{H}_{4} \mathrm{SiO}_{4} / \mathrm{L}$ for binder glass. The difference between the two glasses is that the PC glass was heated to about $915^{\circ} \mathrm{C}$, annealed at about $500^{\circ} \mathrm{C}$, cast as an ingot, then crushed and sieved. The as-received glass was only sieved. We have examined the PC glass with SEM/EDS and transmission electron microscopy and have analyzed it with ${ }^{11} \mathrm{~B}$ nuclear magnetic resonance spectroscopy to detect any changes in the composition or microstructure of the glass, i.e., to look for glass/glass phase separation. It was suspected that that a soluble boron-rich phase formed microinclusions in the glass. This was thought possible because the as-received glass has a lower vitrification temperature and was probably manufactured at a temperature $<915^{\circ} \mathrm{C}$. However, no evidence for glass/glass phase separation was found.

From the results in Figure 19a, the apparent solubility limit for the advanced PC CWF is higher than that for the HIP CWF: about $210 \mathrm{mg} \mathrm{H}_{4} \mathrm{SiO}_{4} / \mathrm{L}$ for the PC CWF, compared to about $150 \mathrm{mg} \mathrm{H}_{4} \mathrm{SiO}_{4} / \mathrm{L}$ for the HIP CWF. The apparent solubility limit of a two-phase material is intermediate between the solubilities of the two phases, and depends on the relative surface areas, solubilities, and dissolution rates of the two materials. Long-term PCTs with sodalite have shown it to have a solubility of about 106 $\mathrm{mg} \mathrm{H}_{4} \mathrm{SiO}_{4} / \mathrm{L}$ (those data are not included in this report). The apparent solubility limit of the CWF is intermediate between that of the binder glass, which accounts for about $25 \%$ of the exposed surface area, and sodalite, which accounts for about $75 \%$ of the exposed surface area. The finding that the apparent solubility limit of the PC CWF is higher than that of the HIP CWF is consistent with PC glass having a higher solubility limit than asreceived glass, since these are representative of the glass phases in the PC and HIP CWF materials.

\subsection{EFFECTS OF PROCESSING CONDITIONS}

A series of PC CWF materials referred to as temperature-time PC CWFs (T-t PC CWFs) were made using the same as-batched compositions but different processing temperatures and hold times. The correspondence between the sample identifier "NLS-\#" and the processing conditions is summarized in Table 31. These materials were analyzed to determine the effects of these processing parameters on the physical and chemical nature of the CWF. These results provide insight that may well be used to select the optimal parameter values for producing CWF products. While the 20 -g size of 
the T-t PC CWF products ensured they were well equilibrated, the larger size of the CWF waste forms will result in significant temperature gradients and times-attemperature. These results can be used with models of heat transfer for the full-sized waste forms to select processing conditions that will result in an acceptable waste form. For example, the processing temperature and time should be selected to ensure that the center of the waste form reaches a sufficiently high temperature while the outside of the waste form does not reach too high a temperature. The tests and analyses discussed below were performed using the 20 -g T-t PC CWF products.

\subsubsection{Physical Characterization}

\subsubsection{Density}

The bulk densities were measured using octanol as the suspension fluid. The densities measured for the $36 \mathrm{~T}-\mathrm{t}$ PC CWF products are given in Table 32. In general, the bulk density increased with processing temperature and with the hold time at a given temperature. The $850^{\circ} \mathrm{C}-1-\mathrm{h} \mathrm{CWF}$ product had the lowest density. It was chalk-like and easily crushed. Products made at temperatures of $900^{\circ} \mathrm{C}$ and higher were mechanically strong and difficult to crush. Product density increased with increasing hold time at all temperatures except at $950^{\circ} \mathrm{C}$. At $950^{\circ} \mathrm{C}$, the maximum density was obtained with hold times of 4 and $8 \mathrm{~h}$; longer hold times resulted in lower densities. Inspection of the products made at $950^{\circ} \mathrm{C}$ for hold times of 24 and 36 hours showed the glass had flowed to the bottom of the crucible during processing.

\subsubsection{X-Ray Diffraction}

A small amount of material from each of the $36 \mathrm{~T}-\mathrm{t}$ PC CWF products was crushed and analyzed with powder X-ray diffraction to evaluate the effect of process temperature and hold time on the phase assemblages in the PC CWF. Tables 33-38 provide summaries of the results (d-spacing and relative intensity) from the XRD analysis of the 36 products. The results are organized to show the effect of temperature for different processing times. The d-spacings are indexed to reference spectra of sodalite $(\mathrm{S})$, halite $(\mathrm{H})$, and nepheline $(\mathrm{N})$ that were given in Table 6 . The relative intensities indicate that sodalite is the most abundant crystalline phase in all $\mathrm{T}-\mathrm{t}$ PC CWF materials. The two most intense halite lines are at 2.821 and $1.994 \AA$. Nepheline has been detected previously in HIP CWF and in some PC CWF products (see Table 5). The strongest nepheline lines are at 3.834 and $3.000 \AA$. These occur as weak lines in the spectra of the T-t PC CWF materials made at low temperatures and short processing times.

\subsubsection{Scanning Electron Microscopy}

Several samples have been examined with SEM/EDS. Micrographs for two T-t PC CWF products are shown in Figs. 20 and 21. The glass phase appears as dark gray regions in these micrographs and sodalite regions appear as light gray. The small white features that are sparsely distributed throughout the samples are rare earth silicates and/or 
oxide inclusions. The large irregularly shaped voids result from incomplete densification. Figure 20a shows that the sodalite granules are not completely embedded in the glass matrix in the product made at $850^{\circ} \mathrm{C}$ with a 1 -h hold time. Figure $20 \mathrm{~b}$ is a micrograph of the same product at higher magnification that shows that glass has not penetrated between the grains comprising the sodalite granule. These micrographs are consistent with the observation that the bulk $850^{\circ} \mathrm{C}-1-\mathrm{h} \mathrm{CWF}$ product is friable and has low density.

The micrograph for the $915^{\circ} \mathrm{C}-16$-h product NLS-8 in Figure 21 shows more complete densification. The light gray areas are sodalite granules in which the regions between the grains have been infiltrated with glass. Individual sodalite grains are not visible. The sodalite granules are completely embedded in the glass matrix. The black regions are pores and voids. The round pores visible in the figure may have been formed during processing from the evolution of gases (residual water or inert gas trapped between the grains) or during polishing due to "pull-out" and/or dissolution of the halite phase. We estimated the porosity in PC products by dividing the bulk density of the PC products by the density of the crushed material $\left(2340 \mathrm{~kg} / \mathrm{m}^{3}\right)$ and subtracting the quotient from 1.00. For NLS-2, the bulk density is 1160 (from Table 32), and $1.00-$ $1160 / 2340=0.504$, which indicates there is about $50 \%$ porosity in the $850^{\circ} \mathrm{C}-1-\mathrm{h}$ product. For NLS-8, the bulk density is $1950 \mathrm{~kg} / \mathrm{m}^{3}$ and the porosity is about $14 \%$. In Figure 21 , pores account for only about $6 \%$ of the imaged surface.

\subsubsection{Chemical Durability Tests}

Triplicate RWS and triplicate 7-day PCTs were conducted with each of the $36 \mathrm{~T}-\mathrm{t} \mathrm{CWF}$ products. These results were analyzed to evaluate the effects of processing conditions on the abundance of halite and the durability of the waste forms.

\subsubsection{Releases in RWS and PCT Steps}

The individual results for the RWS and PCT parts provide insight into the dissolution of the halite separate from the dissolution of the sodalite and binder glass. The test data are given in Table 39. The measured concentrations and calculated $N L(i)$ values are given in Tables 40-45. In Figs. 22-27, the averages of the $N L(i)$ values for the RWS and PCT parts are shown separately for each element. The results are grouped by hold time. As observed in tests with other CWF materials, most of the $\mathrm{Cl}$, I, and $\mathrm{Na}$ released in the test occurs in the RWS step due to dissolution of halite. The values for the RWS fractions of $N L(\mathrm{I})$ and $N L(\mathrm{Na})$ are plotted against $N L(\mathrm{Cl})$ in Figure 28 . The results of tests with all T-t PC CWF processed for hold times of $1 \mathrm{~h}$ and at $950^{\circ} \mathrm{C}$ for 24 and $36 \mathrm{~h}$ have been excluded from the plot because these materials were not acceptably consolidated. The releases of $\mathrm{Cl}$, I, and $\mathrm{Na}$ in the RWS are well correlated. The releases of $\mathrm{Na}$ and $\mathrm{Cl}$ are expected to be correlated because the compositions of the RWS solutions are dominated by the dissolution of halite $(\mathrm{NaCl})$. The observation that the release of $\mathrm{I}$ is also correlated with $\mathrm{Cl}$ (and $\mathrm{Na}$ ) indicates that the concentration of $\mathrm{I}$ is the same in halite formed under all temperature-hold time processing conditions. Furthermore, the slope of the correlation between $\mathrm{I}$ and $\mathrm{Cl}$ is about 1, which indicates that the same fraction of the total amounts of $\mathrm{I}$ and $\mathrm{Cl}$ in the $\mathrm{CWF}$ are in the halite 
phases. A smaller fraction of the total amount of $\mathrm{Na}$ than $\mathrm{Cl}$ in the $\mathrm{CWF}$ is in the halite phase. The RWS values of $N L(\mathrm{Cs})$ and $N L(\mathrm{Li})$ were found not to be correlated with $N L(\mathrm{Cl})$; they had correlation coefficients of $\mathrm{R}^{2}=0.009$ and 0.006 , respectively.

\subsubsection{Halite Content from XRD}

The $\mathrm{Cl}$ ion concentrations that are measured in the RWS test solutions can be used as an indicator of the abundance of halite at the surface of the crushed material. The response may also provide a measure of the total amount of halite in the PC CWF. As discussed previously, crushed (not sieved) material from each of the $36 \mathrm{~T}-\mathrm{t}$ PC CWF products was analyzed with XRD to measure the phase assemblage. Halite peaks were seen in the spectra of all products. The spectra were analyzed to quantify the amount of halite in the bulk CWF. The mass\% of halite in each of the 36 products was estimated using the observed intensities compared according to the relative intensity ratios for sodalite and halite reference materials in the International Centre for Diffraction Data [7]. In Figure 29, the percentage of chloride in the crushed T-t PC CWF that dissolved in the RWS test is plotted against the mass fraction of halite measured by XRD. Neither the amount of halite detected by XRD nor the amount of $\mathrm{Cl}$ in the RWS solution shows a general trend with processing temperature or hold time. There is a fairly large amount of scatter in the XRD results due to the small amount of halite in the CWF and the resulting low signal-to-noise ratio in the XRD spectrum. The detection limit for most crystalline phases in XRD analysis is on the order of 5\%; the measured halite contents in the CWF products are all less than 5\%.

The correlation between the RWS and XRD results is important and meaningful, but the comparison of the values is not. This is because the two methods measure different things: the RWS step measures the fraction of $\mathrm{Cl}$ present in halite and at the outer surface of the particles. The entire volume of the crushed particles is probed by $\mathrm{XRD}$, and the analysis estimates the percentage of the crystalline phases that is halite. The analysis ignores the approximately $25 \%$ of the CWF volume that is X-ray amorphous glass. Therefore, the XRD results that less than $5 \%$ of the crystalline phases is halite means that the halite content in the CWF is less than $75 \% \cdot 5 \%=3.75 \%$. The RWS test measures the amount of $\mathrm{Cl}$ present in halite exposed at the surface, which is then expressed as a percentage of the total chloride initially present in the entire particle based on the mass of material used in the test. Most of the $\mathrm{Cl}$ in the CWF is part of the sodalite structure. We estimate that the $\mathrm{Cl}$ dissolved in the RWS test comes from less than $3 \%$ of the particle volume. This is based on the assumption that the halite inclusions penetrate an average of $1 \mu \mathrm{m}$ into the particles of crushed PC CWF and that the particles have a diameter of $112 \mu \mathrm{m}$. Additional $\mathrm{Cl}$ is released in the PCT step due to dissolution of halite beneath the original near surface and dissolution of sodalite. However, there was no significant difference in the correlation plot when the XRD results were plotted against the sum of the releases of $\mathrm{Cl}$ in the RWS and PCT steps. 


\subsubsection{Total Release}

The mean values of $N L(\mathrm{~B}), N L(\mathrm{Cl}), N L(\mathrm{Na})$, and $N L(\mathrm{Si})$ for the triplicate PCTs were compared to determine the effect of processing conditions on the releases in the PCTs. The sums of the $N L(i)$ values for the RWS and PCT steps for $\mathrm{B}, \mathrm{Si}, \mathrm{Cl}$, and $\mathrm{Na}$ are summarized in Table 46. Values are plotted against the processing temperature for hold times between 4 and 36 hours in Figures. 30a-d. The results for products made with a 1-h hold time are excluded from further consideration because those products were not adequately consolidated. The value of $N L(\mathrm{Si})$ decreases slightly with increasing process temperatures for all processing hold times. The values of $N L(\mathrm{~B})$ decrease for products made at increasing process temperature for hold times of 4 and 8 hours, but increase slightly with temperature for products made using longer hold times. The values of $N L(\mathrm{Cl})$ and $N L(\mathrm{Na})$ increase with the processing temperature for all hold times. As seen in Figs. 22-27, the releases of B and Si occur primarily in the PCT step, whereas the releases of $\mathrm{Cl}$ and $\mathrm{Na}$ occur primarily in the RWS step. This suggests that the increase in the releases of $\mathrm{Cl}$ and $\mathrm{Na}$ are due to an increase in the amount of halite that is formed with the process temperature. The chemical durabilities of the PC CWF materials are best reflected in the values of $N L(\mathrm{~B})$ and $N L(\mathrm{Si})$. The values of $N L(\mathrm{~B})$ and $N L(\mathrm{Si})$ for products made at the same process temperature but different hold times are fairly similar and do not show obvious trends with regard to the hold time, except for the greater releases of B for products made with hold times of 4 hours or less that was noted earlier. Overall, the results of these tests and analyses indicate that the primary effect of processing conditions is on the abundance of halite inclusions that are formed. 


\section{SUMMARY}

Several series of tests and analyses were conducted to evaluate waste forms made by pressureless consolidation and compare the physical and chemical properties of the PC CWF materials to CWFs made using a HIP process. Physical characterizations included measurement of the bulk density, identification of crystalline phases using $\mathrm{XRD}$, and examination of the microstructure with SEM. Chemical characterizations included AFSM, RWS, MCC-1 tests, and PCTs conducted for 7 days to evaluate the effects of CWF composition and processing conditions and for up to 1 year to evaluate the long-term dissolution behavior. Other tests were conducted to evaluate the reproducibility of making PC CWF on the laboratory scale and of running 7-day PCTs. The results of these tests and analyses show subtle differences in the chemical durabilities of CWF materials made with different glass-to-SLZ mass ratios and different processing conditions. These results provide insight that can be used to optimize the PC CWF with respect to waste loading, processing conditions, density, and chemical durability. In general, these tests show the PC CWF to be an acceptable alternative to the HIP CWF with regard to relevant physical and chemical properties. Specific findings are summarized below:

- PC CWFs have lower bulk densities than HIP CWFs. This is due to relatively large voids (up to about $50 \mu \mathrm{m}$ ) that remain mostly in the binder glass. The densities of the crushed PC CWF materials used in the PCT were the same as the density of the HIP CWF. (See Section 4.1.1.)

- The composition of the PC CWF is consistent with the as-batched composition. Heating the glass/SLZ mixture to $>900^{\circ} \mathrm{C}$ without containment did not result in a significant loss of volatile components, such as Cs. Different size fractions of crushed PC CWF had the same compositions, which indicates that the major phases (sodalite, binder glass, and halite) are not fractionated due to crushing and sieving. (See Section 4.1.2.)

- PC CWF materials made with different glass/SLZ mixtures and under different processing conditions have the same major phase assemblage as the HIP CWF. Sodalite is the principal crystalline phase in both PC and HIP CWF materials. Small differences in the amounts of halite and nepheline occur for different processing conditions. (See Section 4.1.3.)

- PC CWFs and HIP CWFs have the same microstructure. All CWF materials consist of $100-\mu \mathrm{m}$ size sodalite domains fixed in a continuum of binder glass. Small crystallites of halite and rare earth oxides are present as inclusions in the binder glass. In the HIP CWF, these are segregated near the sodalite domains, whereas they are more evenly distributed throughout the glass in the PC CWF. The PC CWF contains fairly large voids (up to about $50 \mu \mathrm{m}$ ) mostly in the glass. The HIP CWF contains small voids in the glass near the sodalite interfaces. (See Section 4.1.4.)

- The RWS test provides information regarding the relative amount of halite in the PC CWF. This is equivalent to the AFSM that was conducted with the HIP CWF. Dissolution of halite inclusions exposed at the surfaces of crushed CWF dissolve immediately when contacted by water. The RWS test is 
conducted during preparation of the CWF for the PCT. (See Sections 4.2.1 and 4.2.2.)

- Dissolution of HIP CWF and PC CWF are the same under dilute conditions. Short-term MCC-1 tests highlight dissolution in the absence of solution feedback effects and are dominated by the dissolution of sodalite. The test responses of HIP CWF and PC CWF are similar in MCC-1 tests. MCC-1 tests conducted at $125^{\circ} \mathrm{C}$ for about 3 months show preferential dissolution at the glass/sodalite interface for both materials. (See Section 4.2.3.)

- The PCT responses for samples taken from various locations in a PC CWF product are uniform within the repeatability of the PCT. The responses of PCTs conducted with samples taken from different areas of large PC products were within the repeatability of PCTs conducted with a consolidated mixture.

- The RWS solution is dominated by dissolution of halite; the PCT solution is dominated by dissolution of sodalite and binder glass. For all CWF materials, the RWS solution contains equimolar amounts of $\mathrm{Na}$ and $\mathrm{Cl}$ from the dissolution of halite. Small amounts of other elements that are contaminants in the halite, I, are also present in the RWS solutions. Dissolution of binder glass and sodalite lead to high concentrations of $\mathrm{Al}, \mathrm{B}, \mathrm{Si}$, and $\mathrm{Na}$ in the PCT solution. (See Sections 4.3.1 through 4.3.4.)

- Laboratory-scale PC CWF products can be made reproducibly. The PCT responses of 10 identical products made on different days are within the repeatability of replicate tests conducted with a consolidated mixture. (See Section 4.3.2.)

- The B response in 7-day PCTs can be used to monitor the glass/SLZ loading ratio. The $\mathrm{B}$ responses in PC CWFs made with 25, 30, 35, 40, and 45 mass\% binder glass were distinguishable. (See Section 4.3.3.)

- The PC CWF can be consolidated with 25 mass\% binder glass and 75 mass\% SLZ. Processing at temperatures between about 900 and $925^{\circ} \mathrm{C}$ result in acceptable consolidation and chemical durability. (See Section 4.3.4.)

- The same precision can be achieved in PCTs conducted with PC CWFs as with HLW glasses. The standard deviations of the $\mathrm{Al}, \mathrm{B}, \mathrm{Na}$, and $\mathrm{Si}$ concentrations in 7-day PCTs with advanced PC CWFs were within the ranges measured in inter-laboratory studies of 7-day PCTs with borosilicate glasses. The 7-day PCT can be used to meet the WASRD requirement to monitor the consistency of waste form products. (See Section 4.4.2.)

- The 7-day PCT responses of all CWF materials are significantly lower than those of EA glass. The concentrations of the same elements B, Na, and Si can be used to track the consistency of the PC CWF and HLW glasses. The WASRD requires the response of HLW glasses to be less than that of the EA glass. The responses of PC CWF materials are less than the response of the EA glass, so the PC CWF meets the chemical durability requirement in the WASRD. (See Section 4.4.3.)

- HIP CWFs and PC CWFs have similar long-term durabilities. PCTs conducted for up to 1 year show similar releases of B and Si, which are used to track the dissolution of binder glass and sodalite, respectively. (See Section 4.5.1.) 
- The release of $B$ from the binder glass phase in the PC CWF is significantly lower than that from the binder glass alone. Long-term PCTs with samples of the binder glass showed much higher releases of $\mathrm{B}$ than were measured in parallel tests with advanced PC CWFs or HIP CWFs. No evidence of phase separation could be detected in the binder glass. (See Section 4.5.3.)

- The apparent Si solubility limit is similar for the PC CWF and HIP CWF. The concentrations attained in long-term PCTs are used to estimate the apparent solubility limit, which is a parameter in the CWF degradation model. The highest concentrations attained in tests at 2300 an $23,000 \mathrm{~m}^{-1}$ were about 200 and $150 \mathrm{mg} \mathrm{H} \mathrm{SiO}_{4} / \mathrm{mL}$ for PC CWF, respectively. The concentrations in tests with HIP CWF were about 140 and $150 \mathrm{mg} \mathrm{H}_{4} \mathrm{SiO}_{4} / \mathrm{mL}$ at these $S / V$ ratios. (See Section 4.5.4.)

- The apparent solubility limits for binder glass were higher than those for CWF materials. The highest concentrations attained in tests with the asreceived binder glass were about $280 \mathrm{mg} \mathrm{H}_{4} \mathrm{SiO}_{4} / \mathrm{mL}$ in tests at $2300 \mathrm{~m}^{-1}$ and about $430 \mathrm{mg} \mathrm{H} \mathrm{H}_{4} \mathrm{SiO}_{4} / \mathrm{mL}$ in tests at $23,000 \mathrm{~m}^{-1}$. The CWF degradation model currently uses the highest concentration achieved in tests with binder glass alone to model the degradation of the CWF for conservatism. (See Section 4.5.4.)

- The consolidation of PC CWF materials is sensitive to the processing temperature and processing time. The bulk density increased with the processing temperature and the processing time. Materials processed for only one hour were not adequately consolidated, whereas materials processed for $24 \mathrm{~h}$ or more at $950^{\circ} \mathrm{C}$ phase separated. (See Section 4.6.1.)

- Excessive processing times result in the dissolution of sodalite into the binder glass. This was concluded from visual inspection of the sizes of the sodalite domains after different processing times at $850^{\circ} \mathrm{C}$. (See Section 4.3.5.)

- The amount of halite formed varies with the processing temperature and processing time. The halite content was estimated from XRD results and from the RWS test results. A fairly good correlation was seen for the two methods. However, the amount of halite was not proportional to either the processing temperature or time. (See Section 4.6.2.2.)

- The concentrations of $\mathrm{Cl}, \mathrm{Na}$, and $\mathrm{I}$ in the RWS fraction are correlated. The releases in the RWS step are related as follows: $N L(\mathrm{Na}) / N L(\mathrm{Cl})=0.21$ with a correlation coefficient $\mathrm{R}^{2}=0.96$ and $N L(\mathrm{I}) / N L(\mathrm{Cl})=1.07$ with a correlation coefficient $\mathrm{R}^{2}=0.72$. The correlation between $\mathrm{Cl}$ and I provides a convenient means of tracking the release of I from the RWS results. (See Section4.6.2.1.)

- The durability of PC CWF materials increases with the processing temperature, but is not strongly depended on the processing time (for times greater than $1 \mathbf{h})$. The values of $N L(\mathrm{Si})$ decrease slightly as the processing temperature increases. The values of $N L(\mathrm{~B})$ are similar for different processing conditions (except for PC CWF processed at $850^{\circ} \mathrm{C}$ and at all temperatures for 1 $\mathrm{h}$, which are not acceptably consolidated). (See Section 4.6.2.3.) 


\section{REFERENCES}

1. "Record of Decision for the Treatment and Management of Sodium-Bonded Spent Nuclear Fuel," Federal Register, 65 (182), pp. 56565-56570 (September 19, 2000).

2. L. R. Morss, S. G. Johnson, W. L. Ebert, T. DiSanto, S. M. Frank, J. L. Holly, A. J. Kropf, C. J. Mertz, M. Noy, T. P. O'Holleran, M. K. Richmann, W. Sinkler, Y. Tsai, and A. R. Warren, Corrosion Tests with Uranium- and Plutonium-Loaded Ceramic Waste Forms, Argonne National Laboratory Report, ANL-02/09 (2002).

3. American Society for Testing and Materials, "Standard Test Method for Determining Chemical Durability of Nuclear Waste Glasses: The Product Consistency Test (PCT)," Annual Book of ASTM Standards, 12.01, C1285-94, pp. 796-813 (1996).

4. Waste Acceptance System Requirements Document (WASRD), Rev. 3, DOE/RW0351. U.S. Department of Energy, Office of Civilian Radioactive Waste Management Report E00000000-00811-1708-00001 (April 1999).

5. American Society for Testing and Materials, "Standard Test Method for Static Leaching of Monolithic Waste Forms for Disposal of Radioactive Waste," Annual Book of ASTM Standards, 12.01, C1220-98, pp. 1-16 (1998).

6. A. M. Essling, E. A. Huff, and D. G. Graczyk, "Innovative Methods for Inorganic Sample Preparation," Argonne National Laboratory Report, ANL/ACL-92-1 (1992).

7. Powder Diffraction File, Release 1999 [CD-ROM]; Ed., W. F. McClune, International Centre for Diffraction Data, Newtown Square, PA (1999).

8. W. L. Ebert, M. A. Lewis, and S. G. Johnson, J. Nucl. Mater. 305, 37-51 (2002).

9. American Society for Testing and Materials, "Standard Practice for Conducting an Interlaboratory Study to Determine the Precision of a Test Method," Standard E 691, Annual Book of ASTM Standards, 14.02, E691-99, pp. 264-285 (2000).

10. W. L. Ebert, S. F. Wolf, J. Nucl. Mater. 282, 112-124 (2000).

11. G. F. Piepel, T. E. Jones, D. L. Eggett, G. B. Mellinger, Product Consistency Test Round Robin Conducted by the Materials Characterization Center-Summary Report, Pacific Northwest National Laboratory Report PNL-6967 (1989).

12. M. K. Andrews and N. E. Bibler, "Radioactive Demonstration of DWPF Product Control Strategy," Ceram. Trans., 39, Environmental and Waste Management Issues in the Ceramic Industry, pp. 205-221 (1993). 
Table 1. Constituents of Simulated 300-Driver Salt

\begin{tabular}{|c|c|c|c|c|c|}
\hline Salt & Mass \% & Salt & Mass \% & Salt & Mass \% \\
\hline $\mathrm{BaCl}_{2}$ & 1.20 & $\mathrm{KI}$ & 0.15 & $\mathrm{PrCl}_{3}$ & 1.15 \\
\hline $\mathrm{CeCl}_{3}$ & 2.33 & $\mathrm{LaCl}_{3}$ & 1.22 & $\mathrm{RbCl}$ & 0.33 \\
\hline $\mathrm{CsCl}$ & 2.51 & $\mathrm{LiCl}-\mathrm{KCl}^{\mathrm{a}}$ & 69.80 & $\mathrm{SmCl}_{3}$ & 0.69 \\
\hline $\mathrm{EuCl}$ & 0.05 & $\mathrm{NaCl}$ & 14.95 & $\mathrm{SrCl}_{2}$ & 1.00 \\
\hline $\mathrm{KCl}$ & 0.02 & $\mathrm{NdCl}_{3}$ & 3.90 & $\mathrm{YCl}_{3}$ & 0.70 \\
\hline
\end{tabular}

${ }^{\mathrm{a}}$ Eutectic salt. 
Table 2. Measured Densities of Crushed and Bulk CWF Materials, in $\mathrm{g} / \mathrm{cm}^{3}$

\begin{tabular}{|c|c|c|c|}
\hline Material & Size Fraction & Density $^{a}$ & Bulk Density \\
\hline \multirow{8}{*}{$\begin{array}{l}\text { CD08201 } \\
\text { (5-kg baseline PC CWF) }\end{array}$} & \multicolumn{2}{|c|}{ Measured in Demineralized Water } & \multirow{8}{*}{2.17} \\
\hline & -325 & $2.27 \pm 0.05(5)$ & \\
\hline & $-200+325$ & $2.30 \pm 0.02(4)$ & \\
\hline & $-40+100$ & $2.29 \pm 0.02(2)$ & \\
\hline & \multicolumn{2}{|c|}{ Measured in Octanol } & \\
\hline & -325 & $2.39(1)$ & \\
\hline & $-40+100$ & $2.37(1)$ & \\
\hline & -325 & $2.39(1)$ & \\
\hline \multirow{9}{*}{$\begin{array}{l}\text { PC } 03301 \\
\text { (advanced PC CWF) }\end{array}$} & \multicolumn{2}{|c|}{ Measured in Demineralized Water } & \multirow{9}{*}{1.94} \\
\hline & -325 & $2.45(1)$ & \\
\hline & $-200+325$ & $2.38(1)$ & \\
\hline & $-100+200$ & $2.36(2)$ & \\
\hline & $-80+100$ & $2.27 \pm 0.08(6)$ & \\
\hline & $-20+60$ & $2.37(1)$ & \\
\hline & \multicolumn{2}{|c|}{ Measured in Octanol } & \\
\hline & $-200+325$ & $2.36 \pm 0.08(3)$ & \\
\hline & $-20+60$ & $2.44(1)$ & \\
\hline PC-25\% glass & -325 & $2.38(1)$ & $1.44(2)$ \\
\hline PC-30\% glass & -325 & $2.26 \pm 0.02(2)$ & $1.52(2)$ \\
\hline PC-35\% glass & -325 & $2.27(1)$ & $1.81(2)$ \\
\hline PC-40\% glass & -325 & $2.31(1)$ & $1.97(2)$ \\
\hline PC-45\% glass & -325 & $2.33(1)$ & not measured \\
\hline Reference waste glass & $-100+200$ & $2.69(1)$ & 2.68 \\
\hline HIP CWF & $-100+200$ & $2.35 \pm 0.06(4)$ & 2.35 \\
\hline
\end{tabular}

${ }^{\mathrm{a}}$ Mean \pm 1 standard deviation. Number of replicate analyses is given in parenthesis. 
Table 3. Average Compositions of Baseline PC, Advanced PC, HIP, and As-Batched CWF Materials, in mass\%

\begin{tabular}{|c|c|c|c|c|c|c|c|c|c|c|}
\hline \multirow[b]{2}{*}{ Element } & \multirow[b]{2}{*}{$\begin{array}{c}\text { Binder } \\
\text { Glass }\end{array}$} & \multirow[b]{2}{*}{ Salt } & \multirow[b]{2}{*}{ Zeolite } & \multicolumn{2}{|c|}{ CWF with $50 \%$ glass } & \multicolumn{5}{|c|}{ CWF with $25 \%$ glass } \\
\hline & & & & As-Batched ${ }^{2}$ & $\begin{array}{l}\text { Baseline } \\
\text { PC CWF }\end{array}$ & $\begin{array}{c}\text { As- } \\
\text { Batched }^{b}\end{array}$ & $\begin{array}{l}\text { Advanced } \\
\text { PC CWF }^{c}\end{array}$ & $\begin{array}{l}\text { Advanced } \\
\text { PC CWF }^{d}\end{array}$ & HIP CWF & HIP CWF \\
\hline $\mathrm{Li}$ & $<0.002$ & 5.5 & $-\mathrm{-}^{\mathrm{e}}$ & 0.29 & 0.27 & 0.44 & 0.38 & 0.40 & 0.42 & 0.42 \\
\hline$B$ & 6.0 & -- & -- & 3.00 & 2.67 & 1.50 & 1.41 & 1.45 & 1.08 & 1.43 \\
\hline $\mathrm{Na}$ & 4.8 & 5.2 & 14.9 & 9.33 & 9.43 & 11.6 & 11.7 & 12.9 & 11.8 & 12.1 \\
\hline $\mathrm{Al}$ & 4.0 & -- & 18.8 & 10.4 & 9.17 & 13.6 & 12.6 & 12.5 & 12.7 & 13.8 \\
\hline $\mathrm{Si}$ & 29.5 & -- & 18.7 & 23.1 & 23.7 & 19.9 & 20.1 & -- & 20.6 & 18.8 \\
\hline $\mathrm{Cl}$ & -- & 59.5 & -- & 3.18 & 3.08 & 4.77 & 5.97 & 4.53 & 4.77 & 3.62 \\
\hline $\mathrm{K}$ & 0.37 & 21.1 & 0.04 & 1.33 & 1.40 & 1.81 & 1.65 & 1.76 & 1.68 & 1.74 \\
\hline $\mathrm{Rb}$ & -- & 0.23 & -- & 0.012 & 0.014 & 0.018 & -- & -- & 0.016 & 0.015 \\
\hline $\mathrm{Sr}$ & 0.02 & 0.54 & 0.00 & 0.040 & 0.053 & 0.050 & -- & -- & 0.046 & 0.040 \\
\hline$Y$ & -- & 0.19 & -- & 0.010 & 0.016 & 0.015 & -- & -- & 0.021 & 0.020 \\
\hline $\mathrm{I}$ & -- & 0.12 & -- & 0.0064 & 0.0061 & 0.0096 & 0.0080 & 0.0041 & 0.011 & 0.0045 \\
\hline Cs & -- & 1.82 & -- & 0.097 & 0.11 & 0.15 & 0.15 & 0.16 & 0.15 & 0.15 \\
\hline $\mathrm{Ba}$ & 0.022 & 0.79 & 0.013 & 0.060 & 0.435 & 0.078 & -- & -- & 0.07 & 0.08 \\
\hline La & -- & 0.64 & -- & 0.034 & 0.086 & 0.0511 & -- & -- & 0.060 & 0.040 \\
\hline $\mathrm{Ce}$ & -- & 1.22 & -- & 0.065 & 0.112 & 0.0981 & -- & -- & 0.113 & 0.080 \\
\hline $\mathrm{Pr}$ & -- & 0.54 & -- & 0.029 & 0.074 & 0.0430 & -- & -- & 0.077 & 0.050 \\
\hline $\mathrm{Nd}$ & 0.005 & 1.96 & -- & 0.11 & 0.088 & 0.16 & -- & -- & 0.17 & 0.15 \\
\hline $\mathrm{Sm}$ & -- & 0.35 & -- & 0.019 & 0.015 & 0.028 & -- & -- & 0.031 & 0.020 \\
\hline $\mathrm{Eu}$ & -- & 0.03 & -- & 0.002 & -- & 0.002 & -- & -- & $<0.01$ & $<0.002$ \\
\hline
\end{tabular}

${ }^{\mathrm{a}}$ Batched as 50 mass $\%$ binder glass, 5.4 mass \% salt, and 44 mass $\%$ zeolite.

${ }^{\mathrm{b}}$ Batched as 25 mass $\%$ binder glass, 8.0 mass \% salt, and 67 mass $\%$ zeolite.

${ }^{\mathrm{c}}$ Advanced PC CWF material 02701.

${ }^{\mathrm{d}}$ Advanced PC CWF material 03301.

e-- means not measured. 
Table 4. Measured Compositions of Different Sieve Fractions, in mass\%

\begin{tabular}{|c|c|c|c|c|c|}
\hline \multicolumn{6}{|c|}{ Baseline PC CWF } \\
\hline Element & Calculated $^{a}$ & $-100+200$ & $-200+325$ & $-200+325$ & $-200+325$ \\
\hline $\mathrm{Li}$ & 0.29 & 0.27 & 0.27 & 0.27 & 0.27 \\
\hline $\mathrm{B}$ & 3.00 & 2.47 & 2.63 & 2.74 & 2.85 \\
\hline $\mathrm{Na}$ & 9.33 & 9.58 & 9.39 & 9.32 & 9.44 \\
\hline $\mathrm{Al}$ & 10.40 & 9.20 & 8.88 & 9.24 & 9.35 \\
\hline $\mathrm{Si}$ & 23.1 & 22.7 & 23.3 & 24.1 & 24.7 \\
\hline $\mathrm{Cl}$ & 3.18 & 3.17 & 3.15 & 2.97 & 3.01 \\
\hline $\mathrm{K}$ & 1.33 & 1.29 & 1.31 & 1.32 & 1.68 \\
\hline \multicolumn{6}{|c|}{ Advanced PC CWF-PC02701 } \\
\hline Element & Calculated $^{b}$ & $-100+200$ & $-100+200$ & & \\
\hline $\mathrm{Li}$ & 0.44 & 0.38 & 0.38 & & \\
\hline $\mathrm{B}$ & 1.50 & 1.40 & 1.42 & & \\
\hline $\mathrm{Na}$ & 11.6 & 11.6 & 11.7 & & \\
\hline $\mathrm{Al}$ & 13.6 & 12.5 & 12.6 & & \\
\hline $\mathrm{Si}$ & 19.9 & 20.2 & 20.0 & & \\
\hline $\mathrm{Cl}$ & 4.77 & 6.06 & 5.88 & & \\
\hline $\mathrm{K}$ & 1.81 & 1.63 & 1.66 & & \\
\hline \multicolumn{6}{|c|}{ Advanced PC CWF-PC03301 } \\
\hline Element & Calculated $^{b}$ & Not Sieved & $-100+200$ & $-200+325$ & -325 \\
\hline $\mathrm{Li}$ & 0.44 & 0.39 & 0.39 & 0.39 & 0.41 \\
\hline $\mathrm{B}$ & 1.50 & 1.36 & 1.45 & 1.46 & 1.50 \\
\hline $\mathrm{Na}$ & 11.6 & 12.8 & 13.0 & 12.9 & 12.9 \\
\hline $\mathrm{Al}$ & 13.6 & 12.0 & 13.0 & 12.8 & 12.4 \\
\hline $\mathrm{Si}$ & 19.9 & $-^{\mathrm{C}}$ & -- & -- & -- \\
\hline $\mathrm{Cl}$ & 4.77 & 4.54 & 4.52 & 4.43 & 4.64 \\
\hline $\mathrm{K}$ & 1.81 & 1.76 & 1.72 & 1.71 & 1.85 \\
\hline
\end{tabular}

${ }^{a}$ Batched as 50 mass $\%$ binder glass, 5.4 mass\% salt, and 44 mass $\%$ zeolite.

${ }^{\mathrm{b}}$ Batched as 25 mass $\%$ binder glass, 8.0 mass $\%$ salt, and 67 mass $\%$ zeolite.

c -- means not measured. 
Table 5. Results of XRD Analysis of PC CWF and HIP CWF Materials

\begin{tabular}{|c|c|c|c|c|c|c|c|c|c|c|}
\hline \multicolumn{2}{|c|}{$\begin{array}{c}\text { Baseline PC } \\
\text { CWF }\end{array}$} & \multicolumn{2}{c|}{$\begin{array}{c}\text { Advanced PC } \\
\text { CWF }\end{array}$} & \multicolumn{2}{c|}{$\begin{array}{c}\text { Glass Loading } \\
\text { PC CWF }\end{array}$} & \multicolumn{2}{c|}{ HIP CWF } & \multicolumn{2}{|c|}{ HIP CWF } & ID $^{\mathrm{a}}$ \\
\hline \multicolumn{2}{|c|}{ DTD $^{\mathrm{a}}$} & \multicolumn{2}{c|}{$\mathrm{PC} 02701^{\mathrm{b}}$} & \multicolumn{2}{c|}{$\mathrm{PC} 25 \%^{\mathrm{c}}$} & & & \\
\hline $\mathrm{d}(\AA)$ & $\mathrm{I}(\%)$ & $\mathrm{d}(\AA)$ & $\mathrm{I}(\%)$ & $\mathrm{d}(\AA)$ & $\mathrm{I}(\%)$ & $\mathrm{d}(\AA)$ & $\mathrm{I}(\%)$ & $\mathrm{d}(\AA)$ & $\mathrm{I}(\%)$ & \\
\hline 6.303 & 29.4 & 6.219 & 27.5 & 6.255 & 24.5 & 6.219 & 28.4 & 6.255 & 29.4 & $\mathrm{~S}$ \\
\hline 4.449 & 6.6 & 4.457 & 6.6 & 4.424 & 6.0 & 4.405 & 7.8 & 4.418 & 7.2 & $\mathrm{~S}$ \\
\hline & & & & & & 4.306 & 1.3 & 4.326 & 1.3 & $\mathrm{~N}$ \\
\hline & & & & 4.185 & 0.7 & 4.165 & 3.4 & 4.173 & 3.4 & $\mathrm{~N}$ \\
\hline & & & & 3.844 & 1.0 & 3.825 & 5.6 & 3.839 & 5.4 & $\mathrm{~N}$ \\
\hline 3.630 & 100.0 & 3.632 & 100.0 & 3.610 & 100.0 & 3.600 & 100.0 & 3.609 & 100.0 & $\mathrm{~S}$ \\
\hline 3.273 & 1.4 & & & & & 3.259 & 5.3 & 3.269 & 4.8 & $\mathrm{H}$ \\
\hline & & & & & & & & & & $\mathrm{N}$ \\
\hline 3.008 & 1.5 & & & & & 2.996 & 8.0 & 3.001 & 7.1 & $\mathrm{~N}$ \\
\hline & & & & & & & & 2.882 & 2.2 & \\
\hline 2.828 & 4.8 & 2.829 & 4.4 & & & 2.815 & 4.4 & & & $\mathrm{~N}$ \\
\hline 2.808 & 7.4 & 2.811 & 8.3 & 2.796 & 7.8 & 2.790 & 7.7 & 2.795 & 7.0 & $\mathrm{~S}$ \\
\hline 2.564 & 16.0 & 2.565 & 18.9 & 2.552 & 16.8 & 2.547 & 19.3 & 2.551 & 17.9 & $\mathrm{~S}$ \\
\hline 2.372 & 17.3 & 2.374 & 18.2 & 2.363 & 18.6 & 2.358 & 17.1 & 2.363 & 16.3 & $\mathrm{~S}$ \\
\hline 2.219 & 1.2 & 2.220 & 1.3 & 2.211 & 0.9 & & & & & $\mathrm{~S}$ \\
\hline 2.092 & 24.8 & 2.092 & 26.3 & 2.085 & 24.3 & 2.081 & 24.9 & 2.083 & 22.5 & $\mathrm{~S}$ \\
\hline 1.997 & 2.2 & 1.999 & 2.3 & 1.977 & 3.8 & 1.994 & 2.0 & 1.993 & 2.1 & $\mathrm{H}$ \\
\hline 1.984 & 3.8 & 1.985 & 4.2 & & & 1.974 & 3.8 & 1.976 & 3.4 & $\mathrm{~S}$ \\
\hline 1.982 & 3.5 & & & & & & & & & \\
\hline & & 1.892 & 3.3 & 1.886 & 3.4 & 1.883 & 3.2 & 1.884 & 3.5 & $\mathrm{~S}$ \\
\hline 1.812 & 2.3 & 1.812 & 2.7 & 1.806 & 2.7 & 1.801 & 2.4 & 1.805 & 2.6 & $\mathrm{~S}$ \\
\hline 1.741 & 2.3 & 1.740 & 3.1 & 1.735 & 3.0 & 1.732 & 2.9 & 1.734 & 2.6 & $\mathrm{~S}$ \\
\hline 1.620 & 1.8 & 1.620 & 2.4 & 1.615 & 2.0 & 1.613 & 2.5 & 1.614 & 1.9 & $\mathrm{~S}$ \\
\hline 1.569 & 7.6 & 1.568 & 8.3 & 1.564 & 8.5 & 1.561 & 8.2 & 1.563 & 7.4 & $\mathrm{~S}$ \\
\hline 1.522 & 3.7 & 1.522 & 3.9 & 1.517 & 3.6 & 1.515 & 4.0 & 1.516 & 3.4 & $\mathrm{~S}$ \\
\hline 1.478 & 6.1 & 1.479 & 6.3 & 1.474 & 5.9 & 1.473 & 5.0 & 1.473 & 4.7 & $\mathrm{~S}$ \\
\hline & & & & & & 1.434 & 5.4 & & & $\mathrm{~S}$ \\
\hline
\end{tabular}

${ }^{a}$ Identification by comparison with reference materials (see Table 6 ): $\mathrm{S}=$ Sodalite, $\mathrm{N}=$ Nepheline, $\mathrm{H}=$ Halite.

${ }^{\mathrm{b}}$ Advanced PC CWF Product 02701.

${ }^{\mathrm{c}}$ Glass loading PC CWF with 20 mass\% glass. 
Table 6. Reference d-Spacings for Sodalite, Halite, and Nepheline ${ }^{a}$

\begin{tabular}{|c|c|c|c|c|c|}
\hline \multicolumn{2}{|c|}{$\begin{array}{c}\text { Sodalite } \\
\text { PDF 37-90476 }\end{array}$} & \multicolumn{2}{|c|}{$\begin{array}{c}\text { Halite } \\
\text { PDF 05-0628 }\end{array}$} & \multicolumn{2}{|c|}{$\begin{array}{c}\text { Nepheline } \\
\text { PDF 35-0424 }\end{array}$} \\
\hline \multirow{2}{*}{$\mathrm{d}(\mathrm{A})$} & $1 \%$ & $\mathrm{~d}(\mathrm{~A})$ & $1 \%$ & $\mathrm{~d}(\mathrm{~A})$ & $1 \%$ \\
\hline & & & & 8.640 & 2.0 \\
\hline \multirow[t]{3}{*}{6.280} & 23.0 & & & & \\
\hline & & & & 6.000 & 4.0 \\
\hline & & & & 4.989 & 6.0 \\
\hline \multirow[t]{4}{*}{4.440} & 4.0 & & & & \\
\hline & & & & 4.321 & 24.0 \\
\hline & & & & 4.280 & 8.0 \\
\hline & & & & 4.165 & 58.0 \\
\hline \multirow[t]{3}{*}{3.970} & 1.0 & & & & \\
\hline & & & & 3.834 & 78.0 \\
\hline & & & & 3.752 & 2.0 \\
\hline \multirow[t]{7}{*}{3.624} & 100 & & & & \\
\hline & & 3.260 & 11.0 & 3.266 & 64.0 \\
\hline & & & & 3.197 & 5.0 \\
\hline & & & & 3.040 & 18.0 \\
\hline & & & & 3.000 & 100 \\
\hline & & & & 2.881 & 36.0 \\
\hline & & 2.821 & 100 & & \\
\hline \multirow[t]{2}{*}{2.807} & 10.0 & & & & \\
\hline & & & & 2.722 & 2.0 \\
\hline \multirow[t]{3}{*}{2.677} & 1.0 & & & & \\
\hline & & & & 2.644 & 3.0 \\
\hline & & & & 2.570 & 58.0 \\
\hline \multirow[t]{5}{*}{2.563} & 23.0 & & & & \\
\hline & & & & 2.496 & 17.0 \\
\hline & & & & 2.426 & 4.0 \\
\hline & & & & 2.397 & 13.0 \\
\hline & & & & 2.390 & 4.0 \\
\hline \multirow[t]{4}{*}{2.373} & 24.0 & & & & \\
\hline & & & & 2.369 & 4.0 \\
\hline & & & & 2.336 & 45.0 \\
\hline & & & & 2.304 & 33.0 \\
\hline \multirow[t]{4}{*}{2.219} & 2.0 & & & & \\
\hline & & & & 2.160 & 7.0 \\
\hline & & & & 2.140 & 6.0 \\
\hline & & & & 2.116 & 10.0 \\
\hline \multirow[t]{5}{*}{2.093} & 35.0 & & & 2.091 & 7.0 \\
\hline & & & & 2.083 & 23.0 \\
\hline & & & & 2.077 & 14.0 \\
\hline & & & & 2.025 & 1.0 \\
\hline & & 1.994 & 78.0 & 1.999 & 3.0 \\
\hline
\end{tabular}

\begin{tabular}{|c|c|c|c|c|c|}
\hline \multicolumn{2}{|c|}{$\begin{array}{c}\text { Sodalite } \\
\text { PDF 37-90476 }\end{array}$} & \multicolumn{2}{|c|}{$\begin{array}{c}\text { Halite } \\
\text { PDF 05-0628 }\end{array}$} & \multicolumn{2}{|c|}{$\begin{array}{c}\text { Nepheline } \\
\text { PDF 35-0424 }\end{array}$} \\
\hline $\mathrm{d}(\mathrm{A})$ & $1 \%$ & $\mathrm{~d}(\mathrm{~A})$ & $1 \%$ & $\mathrm{~d}(\mathrm{~A})$ & $1 \%$ \\
\hline \multirow[t]{5}{*}{1.985} & 5.0 & & & 1.982 & 11.0 \\
\hline & & & & 1.929 & 9.0 \\
\hline & & & & 1.922 & 9.0 \\
\hline & & & & 1.918 & 5.0 \\
\hline & & & & 1.886 & 5.0 \\
\hline \multirow[t]{2}{*}{1.873} & 6.0 & & & 1.876 & 3.0 \\
\hline & & & & 1.839 & 3.0 \\
\hline \multirow[t]{2}{*}{1.812} & 4.0 & & & 1.814 & 3.0 \\
\hline & & & & 1.790 & 8.0 \\
\hline \multirow[t]{2}{*}{1.775} & 2.0 & & & & \\
\hline & & & & 1.756 & 8.0 \\
\hline \multirow[t]{7}{*}{1.741} & 4.0 & & & & \\
\hline & & & & 1.728 & 3.0 \\
\hline & & & & 1.717 & 3.0 \\
\hline & & 1.701 & 3.0 & 1.705 & 5.0 \\
\hline & & & & 1.692 & 9.0 \\
\hline & & & & 1.687 & 4.0 \\
\hline & & & & 1.663 & 4.0 \\
\hline \multirow[t]{3}{*}{1.648} & 2.0 & & & & \\
\hline & & & & 1.635 & 4.0 \\
\hline & & & & 1.633 & 6.0 \\
\hline \multirow[t]{6}{*}{1.621} & 4.0 & 1.628 & 26.0 & & \\
\hline & & & & 1.613 & 15.0 \\
\hline & & & & 1.602 & 6.0 \\
\hline & & & & 1.598 & 8.0 \\
\hline & & & & 1.596 & 6.0 \\
\hline & & & & 1.580 & 2.0 \\
\hline \multirow[t]{4}{*}{1.569} & 12.0 & & & 1.572 & 4.0 \\
\hline & & & & 1.560 & 27.0 \\
\hline & & & & 1.554 & 27.0 \\
\hline & & & & 1.552 & 12.0 \\
\hline 1.545 & 2.0 & & & 1.544 & 10.0 \\
\hline \multirow[t]{2}{*}{1.522} & 7.0 & & & 1.525 & 4.0 \\
\hline & & & & 1.520 & 4.0 \\
\hline 1.500 & 2.0 & & & 1.484 & 12.0 \\
\hline \multirow[t]{6}{*}{1.479} & 10.0 & & & 1.467 & 10.0 \\
\hline & & & & 1.454 & 6.0 \\
\hline & & & & 1.441 & 4.0 \\
\hline & & & & 1.441 & 4.0 \\
\hline & & 1.410 & 12.0 & & \\
\hline & & & & & \\
\hline
\end{tabular}

${ }^{\mathrm{a}}$ Major peaks used for identification shown in bold font. 
Table 7. Results of AFSM with 5-kg Baseline PC CWF

\begin{tabular}{|c|c|c|c|c|}
\hline Test No. & Mass CWF, g & $\begin{array}{c}\text { Volume Water, } \\
\mathrm{mL}\end{array}$ & $(\mathrm{Cl}), \mathrm{mg} / \mathrm{L}$ & $\mathrm{NL}(\mathrm{Cl}), \mathrm{g} / \mathrm{m}^{2}$ \\
\hline \multicolumn{5}{|c|}{ AFSM with Material from Center } \\
\hline 1 & 1.17 & 20.03 & 34.68 & 0.43 \\
\hline 2 & 1.31 & 20.05 & 43.02 & 0.48 \\
\hline 3 & 1.22 & 20.00 & 44.75 & 0.53 \\
\hline Mean $\pm 1 \mathrm{~s}$ & & & & $0.48 \pm 0.05$ \\
\hline \multicolumn{5}{|c|}{ AFSM with Material from Edge } \\
\hline 1 & 1.34 & 20.32 & 45.95 & 0.50 \\
\hline 2 & 1.19 & 20.05 & 45.55 & 0.56 \\
\hline 3 & 1.14 & 20.11 & 41.17 & 0.53 \\
\hline Mean $\pm 1 \mathrm{~s}$ & & & & $0.53 \pm 0.02$ \\
\hline \multicolumn{5}{|c|}{ AFSM with Material from Mid-radius } \\
\hline 1 & 1.19 & 19.98 & 52.66 & 0.64 \\
\hline 2 & 1.35 & 20.08 & 56.01 & 0.60 \\
\hline 3 & 1.09 & 19.96 & 41.35 & 0.55 \\
\hline Mean $\pm 1 \mathrm{~s}$ & & & & $0.60 \pm 0.05$ \\
\hline
\end{tabular}


Table 8. Results of RWS with 5-kg Baseline PC CWF

\begin{tabular}{|c|c|c|c|c|}
\hline Test No. & Mass CWF, g & $\begin{array}{c}\text { Volume Water, } \\
\mathrm{mL}\end{array}$ & $(\mathrm{Cl}), \mathrm{mg} / \mathrm{L}$ & $\mathrm{NL}(\mathrm{Cl}), \mathrm{g} / \mathrm{m}^{2}$ \\
\hline \multicolumn{5}{|c|}{ RWS with CWF-to-Water Ratio 1:10. One Ethanol Wash Prior to RWS } \\
\hline 1 & 1.04 & 10.99 & 55.9 & 0.81 \\
\hline 2 & 0.98 & 10.81 & 55.2 & 0.83 \\
\hline 3 & 1.02 & 10.89 & 8.8 & 0.13 \\
\hline Mean $\pm 1 \mathrm{~s}$ & & & & $0.60 \pm 0.37$ \\
\hline \multicolumn{5}{|c|}{ RWS with CWF-to-Water Ratio 2:20. One Ethanol Wash Prior to RWS } \\
\hline 1 & 2.01 & 20.20 & 44.7 & 0.61 \\
\hline 2 & 1.98 & 19.87 & 49.3 & 0.68 \\
\hline 3 & 1.96 & 19.64 & 46.0 & 0.63 \\
\hline Mean $\pm 1 \mathrm{~s}$ & & & & $0.59 \pm 0.03$ \\
\hline \multicolumn{5}{|c|}{ RWS with CWF-to-Water Ratio 3:30. One Ethanol Wash Prior to RWS } \\
\hline 1 & 2.98 & 30.43 & 38.1 & 0.53 \\
\hline 2 & 2.86 & 28.91 & 44.6 & 0.62 \\
\hline 3 & 2.91 & 30.26 & 37.8 & 0.53 \\
\hline Mean $\pm 1 \mathrm{~s}$ & & & & $0.56 \pm 0.05$ \\
\hline \multicolumn{5}{|c|}{ RWS with CWF-to-Water Ratio 4:40. One Ethanol Wash Prior to RWS } \\
\hline 1 & 3.99 & 39.99 & 45.0 & 0.62 \\
\hline 2 & 3.96 & 39.64 & 46.8 & 0.64 \\
\hline 3 & 3.88 & 38.83 & 43.1 & 0.59 \\
\hline Mean $\pm 1 \mathrm{~s}$ & & & & $0.57 \pm 0.03$ \\
\hline \multicolumn{5}{|c|}{ RWS with CWF-to-Water Ratio 4.5:45. Three 15-mL Ethanol Washes Prior to RWS } \\
\hline & 4.49 & 11.91 & 27.21 & 0.37 \\
\hline \multicolumn{5}{|c|}{ RWS with Consolidated Mixture. Seven Ethanol Washes Prior to RWS } \\
\hline & 38.48 & 321.91 & 27.5 & 0.31 \\
\hline
\end{tabular}


Table 9. Results of MCC-1 Tests with PC CWF and HIP CWF, $N L(i)$ in $\mathrm{g} / \mathrm{m}^{2}$

\begin{tabular}{|c|c|c|c|c|c|c|c|c|}
\hline \multicolumn{2}{|c|}{ Duration, d } & $\mathrm{Al}$ & B & $\mathrm{Cl}$ & Cs & $\mathrm{Li}$ & $\mathrm{Na}$ & $\mathrm{Si}$ \\
\hline \multicolumn{9}{|c|}{ Tests at $90^{\circ} \mathrm{C}$} \\
\hline PC CWF & 1 & 4.58 & 0.788 & 7.46 & $--^{a}$ & 4.16 & 6.18 & 2.29 \\
\hline HIP CWF & 1 & 2.15 & 0.314 & 11.4 & -- & 3.59 & 5.06 & 1.93 \\
\hline PC CWF & 3 & 8.81 & 1.47 & 13.3 & -- & 7.43 & 11.5 & 4.35 \\
\hline HIP CWF & 3 & 4.91 & 0.712 & 16.4 & -- & 8.79 & 9.32 & 4.34 \\
\hline \multicolumn{9}{|c|}{ Tests at $125^{\circ} \mathrm{C}$} \\
\hline PC CWF & 28 & 26.2 & 10.2 & 29.4 & 7.99 & 26.1 & 37.4 & 21.0 \\
\hline HIP CWF & 28 & 15.9 & 10.3 & 25.1 & 30.1 & 20.4 & 27.5 & 19.0 \\
\hline PC CWF & 88 & 20.9 & 22.7 & 45.1 & 17.8 & 53.3 & 33.5 & 22.5 \\
\hline HIP CWF & 88 & 8.49 & 23.8 & 40.7 & 39.9 & 34.2 & 15.9 & 12.8 \\
\hline
\end{tabular}

a---means not measured. 
Table 10. Results of Triplicate Tests with 5-kg Baseline PC CWF, NL(i) in $\mathrm{g} / \mathrm{m}^{2}$

\begin{tabular}{|c|c|c|c|c|}
\hline & Set 1 & Set 2 & Set 3 & Overall \\
\hline \multicolumn{5}{|c|}{$\mathrm{Al}$} \\
\hline RWS & 0.0336 & 0.0336 & 0.0336 & \multirow{5}{*}{$\begin{array}{l}\text { Mean }=0.0941 \\
s=0.0015 \\
\% R S D=1.6 \%\end{array}$} \\
\hline PCT-1 & 0.0907 & 0.0905 & 0.0936 & \\
\hline РCT-2 & 0.0890 & 0.0893 & 0.0899 & \\
\hline PCT-3 & 0.0916 & 0.0898 & 0.0923 & \\
\hline Mean $\pm \mathrm{s}^{\mathrm{a}}$ & $0.0938 \pm 0.0013^{a}$ & $0.0933 \pm 0.0006$ & $0.0953 \pm 0.0018$ & \\
\hline \multicolumn{5}{|c|}{$B$} \\
\hline RWS & 0.0099 & 0.0099 & 0.0099 & \multirow{5}{*}{$\begin{array}{l}\text { Mean }=0.671 \\
\mathrm{~s}=0.013 \\
\% \mathrm{RSD}=1.9 \%\end{array}$} \\
\hline PCT-1 & 0.646 & 0.646 & 0.674 & \\
\hline PCT-2 & 0.650 & 0.657 & 0.683 & \\
\hline PCT-3 & 0.674 & 0.658 & 0.666 & \\
\hline Mean $\pm \mathrm{s}$ & $0.666 \pm 0.015$ & $0.664 \pm 0.006$ & $0.684 \pm 0.009$ & \\
\hline \multicolumn{5}{|c|}{$\mathrm{Cl}$} \\
\hline RWS & 0.315 & 0.315 & 0.315 & \multirow{5}{*}{$\begin{array}{l}\text { Mean }=0.596 \\
\mathrm{~s}=0.017 \\
\% \mathrm{RSD}=2.9 \%\end{array}$} \\
\hline PCT-1 & 0.260 & 0.272 & 0.300 & \\
\hline PCT-2 & 0.281 & 0.267 & 0.296 & \\
\hline PCT-3 & 0.278 & 0.264 & 0.310 & \\
\hline Mean $\pm \mathrm{s}$ & $0.588 \pm 0.011$ & $0.583 \pm 0.004$ & $0.617 \pm 0.007$ & \\
\hline \multicolumn{5}{|c|}{ Cs } \\
\hline RWS & 0.0027 & 0.0027 & 0.0027 & \multirow{5}{*}{$\begin{array}{l}\text { Mean }=0.0252 \\
\mathrm{~s}=0.0017 \\
\% \mathrm{RSD}=1.6 \%\end{array}$} \\
\hline PCT-1 & 0.0215 & 0.0214 & 0.0244 & \\
\hline PCT-2 & 0.0216 & 0.0204 & 0.0247 & \\
\hline PCT-3 & 0.0223 & 0.0215 & 0.0252 & \\
\hline Mean $\pm \mathrm{s}$ & $0.0245 \pm 0.0005$ & $0.0238 \pm 0.0006$ & $0.0274 \pm 0.0004$ & \\
\hline \multicolumn{5}{|c|}{$\mathrm{Li}$} \\
\hline RWS & Not analyzed & Not analyzed & Not analyzed & \multirow{5}{*}{$\begin{array}{l}\text { Mean }=0.695 \\
\mathrm{~s}=0.027 \\
\% \mathrm{RSD}=3.9 \%\end{array}$} \\
\hline PCT-1 & 0.637 & 0.707 & 0.712 & \\
\hline PCT-2 & 0.667 & 0.709 & 0.707 & \\
\hline PCT-3 & 0.689 & 0.714 & 0.713 & \\
\hline Mean $\pm s$ & $0.664 \pm 0.026$ & $0.710 \pm 0.004$ & $0.711 \pm 0.003$ & \\
\hline \multicolumn{5}{|c|}{$\mathrm{Na}$} \\
\hline RWS & 0.057 & 0.057 & 0.057 & \multirow{5}{*}{$\begin{array}{l}\text { Mean }=0.279 \\
\mathrm{~s}=0.012 \\
\% \mathrm{RSD}=4.3 \%\end{array}$} \\
\hline PCT-1 & 0.204 & 0.234 & 0.225 & \\
\hline PCT-2 & 0.205 & 0.234 & 0.223 & \\
\hline PCT-3 & 0.211 & 0.235 & 0.227 & \\
\hline Mean $\pm s$ & $0.264 \pm 0.004$ & $0.291 \pm 0.001$ & $0.282 \pm 0.002$ & \\
\hline \multicolumn{5}{|c|}{$\mathrm{Si}$} \\
\hline RWS & 0.0017 & 0.0017 & 0.0017 & \multirow{5}{*}{$\begin{array}{l}\text { Mean }=0.0759 \\
s=0.0017 \\
\% R S D=2.2 \%\end{array}$} \\
\hline PCT-1 & 0.0733 & 0.0737 & 0.0757 & \\
\hline PCT-2 & 0.0736 & 0.0712 & 0.0772 & \\
\hline PCT-3 & 0.0752 & 0.0735 & 0.0748 & \\
\hline Mean $\pm \mathrm{s}$ & $0.0757 \pm 0.0010$ & $0.0745 \pm 0.0014$ & $0.0776 \pm 0.0011$ & \\
\hline
\end{tabular}

${ }^{\mathrm{a}}$ Mean \pm 1 standard deviation. 
Table 11. Results of 7-Day PCT with DTD PC CWF Materials, $N L(i)$ in $\mathrm{g} / \mathrm{m}^{2}$

\begin{tabular}{|c|c|c|c|c|c|c|c|c|}
\hline Test Number & $\mathrm{Al}$ & B & $\mathrm{Cl}$ & Cs & I & $\mathrm{Li}$ & $\mathrm{Na}$ & $\mathrm{Si}$ \\
\hline \multicolumn{9}{|c|}{ RWS Fraction } \\
\hline RWS-DTD1 & 0.0011 & 0.0054 & 0.821 & 0.00730 & 0.624 & 0.0105 & 0.175 & 0.0015 \\
\hline RWS-DTD2 & 0.0010 & 0.0057 & 0.915 & 0.00864 & 0.719 & 0.0124 & 0.195 & 0.0013 \\
\hline RWS-DTD3 & 0.0020 & 0.0066 & 0.857 & 0.00813 & 0.550 & 0.0233 & 0.201 & 0.0025 \\
\hline RWS-DTD4 & 0.0014 & 0.0052 & 0.814 & 0.00656 & 0.534 & 0.0175 & 0.187 & 0.0013 \\
\hline RWS-DTD5 & 0.0010 & 0.0066 & 0.774 & 0.00726 & 0.500 & 0.0193 & 0.173 & 0.0009 \\
\hline RWS-DTD6 & 0.0028 & 0.0065 & 0.846 & 0.00822 & 0.573 & 0.0215 & 0.199 & 0.0021 \\
\hline RWS-DTD7 & 0.0021 & 0.0066 & 0.667 & 0.00740 & 0.416 & 0.0162 & 0.150 & 0.0017 \\
\hline RWS-DTD8 & 0.0028 & 0.0068 & 0.741 & 0.00750 & 0.494 & 0.0179 & 0.177 & 0.0022 \\
\hline RWS-DTD9 & 0.0272 & 0.0158 & 0.778 & 0.0196 & 0.529 & 0.0354 & 0.206 & 0.0137 \\
\hline RWS-DTD10 & 0.0022 & 0.0062 & 0.753 & 0.00633 & 0.493 & 0.0166 & 0.172 & 0.0021 \\
\hline Mean $^{a}$ & 0.0018 & 0.0062 & 0.7986 & 0.0075 & 0.5449 & 0.0172 & 0.1809 & 0.0017 \\
\hline Std. dev. ${ }^{a}$ & 0.0007 & 0.0006 & 0.0735 & 0.0008 & 0.0876 & 0.0040 & 0.0164 & 0.0005 \\
\hline$\% \mathrm{RSD}^{\mathrm{a}}$ & $40 \%$ & $9.5 \%$ & $9.2 \%$ & $10 \%$ & $16 \%$ & $23 \%$ & $9.0 \%$ & $30 \%$ \\
\hline \multicolumn{9}{|c|}{ PCT Fraction } \\
\hline PPC-DTD-1 & 0.104 & 0.449 & 0.230 & 0.0157 & 0.189 & 0.668 & 0.185 & 0.0706 \\
\hline PPC-DTD-2 & 0.101 & 0.341 & 0.222 & 0.0154 & 0.272 & 0.615 & 0.162 & 0.0679 \\
\hline PPC-DTD-3 & 0.108 & 0.454 & 0.232 & 0.0201 & 0.176 & 0.667 & 0.184 & 0.0694 \\
\hline PPC-DTD-4 & 0.113 & 0.431 & 0.240 & 0.0198 & 0.201 & 0.663 & 0.182 & 0.0711 \\
\hline PPC-DTD-5 & 0.112 & 0.396 & 0.234 & 0.0198 & 0.180 & 0.663 & 0.174 & 0.0696 \\
\hline PPC-DTD-6 & 0.109 & 0.432 & 0.223 & 0.0188 & 0.181 & 0.664 & 0.176 & 0.0704 \\
\hline PPC-DTD-7 & 0.107 & 0.460 & 0.234 & 0.0191 & 0.172 & 0.667 & 0.183 & 0.0714 \\
\hline PPC-DTD-8 & 0.099 & 0.510 & 0.221 & 0.0171 & 0.180 & 0.671 & 0.189 & 0.0679 \\
\hline PPC-DTD-9 & 0.111 & 0.518 & 0.222 & 0.0179 & 0.177 & 0.707 & 0.198 & 0.0749 \\
\hline PPC-DTD-10 & 0.093 & 0.529 & 0.206 & 0.0185 & 0.160 & 0.680 & 0.173 & 0.0688 \\
\hline Mean & 0.105 & 0.445 & 0.227 & 0.018 & 0.190 & 0.662 & 0.178 & 0.070 \\
\hline Std. dev. & 0.006 & 0.056 & 0.010 & 0.002 & 0.033 & 0.018 & 0.008 & 0.001 \\
\hline$\%$ RSD & $6.1 \%$ & $13 \%$ & $4.5 \%$ & $9.6 \%$ & $17 \%$ & $2.8 \%$ & $4.6 \%$ & $1.9 \%$ \\
\hline \multicolumn{9}{|c|}{ Total PCT Response } \\
\hline DTD-1 & 0.105 & 0.454 & 1.05 & 0.0230 & 0.812 & 0.679 & 0.360 & 0.0721 \\
\hline DTD-2 & 0.102 & 0.347 & 1.14 & 0.0241 & 0.992 & 0.627 & 0.356 & 0.0693 \\
\hline DTD-3 & 0.110 & 0.461 & 1.09 & 0.0282 & 0.726 & 0.691 & 0.385 & 0.0718 \\
\hline DTD-4 & 0.114 & 0.436 & 1.05 & 0.0264 & 0.735 & 0.681 & 0.369 & 0.0725 \\
\hline DTD-5 & 0.113 & 0.403 & 1.01 & 0.0271 & 0.680 & 0.682 & 0.347 & 0.0705 \\
\hline DTD-6 & 0.112 & 0.438 & 1.07 & 0.0270 & 0.755 & 0.685 & 0.375 & 0.0725 \\
\hline DTD-7 & 0.109 & 0.467 & 0.90 & 0.0265 & 0.588 & 0.683 & 0.333 & 0.0731 \\
\hline DTD-8 & 0.101 & 0.517 & 0.96 & 0.0246 & 0.674 & 0.688 & 0.366 & 0.0702 \\
\hline DTD-9 & 0.138 & 0.534 & 1.00 & 0.037 & 0.706 & 0.743 & 0.404 & 0.0890 \\
\hline DTD-10 & 0.096 & 0.536 & 0.96 & 0.0248 & 0.654 & 0.697 & 0.344 & 0.0709 \\
\hline Mean & 0.107 & 0.451 & 1.026 & 0.026 & 0.735 & 0.679 & 0.359 & 0.071 \\
\hline Std. dev. & 0.006 & 0.056 & 0.074 & 0.002 & 0.116 & 0.020 & 0.016 & 0.001 \\
\hline \%RSD & $5.9 \%$ & $13 \%$ & $7.2 \%$ & $6.5 \%$ & $16 \%$ & $3.0 \%$ & $4.5 \%$ & $1.8 \%$ \\
\hline
\end{tabular}

${ }^{a}$ Results for DTD-9 are excluded from calculated mean, standard deviation (Std. dev.), and percent relative standard deviation (\%RSD). 
Table 12. Results of PCT with Glass Loading PC CWF Materials, NL(i) in $\mathrm{g} / \mathrm{m}^{2}$

\begin{tabular}{|c|c|c|c|c|c|c|c|c|}
\hline Test Number & $\mathrm{pH}$ & $\mathrm{Al}$ & B & $\mathrm{Cl}$ & Cs & $\mathrm{Li}$ & $\mathrm{Na}$ & $\mathrm{Si}$ \\
\hline \multicolumn{9}{|c|}{$25 \%$ Glass } \\
\hline RWS & & 0.002 & 0.022 & 0.419 & 0.030 & 0.014 & 0.117 & 0.0027 \\
\hline PPC25G-7-1 & 8.81 & 0.100 & 0.176 & $--^{a}$ & 0.032 & 0.455 & 0.114 & 0.0764 \\
\hline PPC25G-7-2 & 8.85 & 0.100 & 0.179 & 0.191 & 0.033 & 0.461 & 0.114 & 0.0758 \\
\hline PPC25G-7-3 & 8.76 & 0.100 & 0.189 & 0.203 & 0.035 & 0.454 & 0.115 & 0.0772 \\
\hline mean total & & 0.102 & 0.203 & 0.616 & 0.063 & 0.471 & 0.232 & 0.0791 \\
\hline Std. Dev. & & 0.0002 & 0.007 & 0.009 & 0.001 & 0.004 & 0.0003 & 0.0007 \\
\hline \multicolumn{9}{|c|}{$30 \%$ Glass } \\
\hline RWS & & 0.001 & 0.015 & 0.536 & 0.015 & 0.012 & 0.120 & 0.0013 \\
\hline PPC30G-7-1 & 8.86 & 0.106 & 0.172 & 0.189 & 0.029 & 0.495 & 0.116 & 0.0785 \\
\hline PPC30G-7-2 & 8.81 & 0.104 & 0.180 & 0.199 & 0.031 & 0.499 & 0.117 & 0.0783 \\
\hline PPC30G-7-3 & 8.56 & 0.102 & 0.181 & 0.208 & 0.031 & 0.490 & 0.119 & 0.0751 \\
\hline Mean total & & 0.105 & 0.192 & 0.735 & 0.045 & 0.506 & 0.238 & 0.0786 \\
\hline Std. dev. & & 0.002 & 0.005 & 0.010 & 0.001 & 0.005 & 0.001 & 0.0019 \\
\hline \multicolumn{9}{|c|}{$35 \%$ Glass } \\
\hline RWS & & 0.002 & 0.016 & 0.783 & 0.014 & 0.012 & 0.167 & 0.0021 \\
\hline PPC35G-7-1 & 8.74 & 0.107 & 0.194 & 0.203 & 0.022 & 0.539 & 0.124 & 0.0762 \\
\hline PPC35G-7-2 & 8.75 & 0.102 & 0.191 & 0.204 & 0.022 & 0.540 & 0.123 & 0.0760 \\
\hline PPC35G-7-3 & 8.52 & 0.103 & 0.186 & 0.216 & 0.023 & 0.530 & 0.124 & 0.0737 \\
\hline Mean total & & 0.106 & 0.207 & 0.991 & 0.036 & 0.548 & 0.291 & 0.0774 \\
\hline Std. dev. & & 0.003 & 0.004 & 0.007 & 0.001 & 0.005 & 0.001 & 0.0014 \\
\hline \multicolumn{9}{|c|}{$40 \%$ Glass } \\
\hline RWS & & 0.001 & 0.007 & 0.852 & 0.012 & 0.013 & 0.192 & 0.0013 \\
\hline PPC40G-7-1 & 8.75 & 0.107 & 0.233 & 0.229 & 0.019 & 0.561 & 0.137 & 0.0733 \\
\hline PPC40G-7-2 & 8.62 & 0.108 & 0.228 & 0.228 & 0.018 & 0.554 & 0.140 & 0.0717 \\
\hline PPC40G-7-3 & 8.64 & 0.105 & 0.206 & 0.227 & 0.017 & 0.530 & 0.138 & 0.0698 \\
\hline mean total & & 0.108 & 0.229 & 1.08 & 0.030 & 0.562 & 0.331 & 0.0729 \\
\hline Std. Dev. & & 0.001 & 0.014 & 0.001 & 0.001 & 0.016 & 0.001 & 0.0017 \\
\hline \multicolumn{9}{|c|}{$45 \%$ Glass } \\
\hline RWS & & 0.001 & 0.006 & 1.009 & 0.009 & 0.011 & 0.213 & 0.0015 \\
\hline PPC45G-7-1 & 8.56 & 0.114 & 0.270 & 0.211 & 0.012 & 0.585 & 0.156 & 0.0715 \\
\hline PPC45G-7-2 & 8.53 & 0.112 & 0.277 & 0.217 & 0.015 & 0.592 & 0.152 & 0.0719 \\
\hline PPC45G-7-3 & 8.66 & 0.111 & 0.253 & 0.235 & 0.015 & 0.565 & 0.152 & 0.0703 \\
\hline Mean total & & 0.113 & 0.273 & 1.23 & 0.023 & 0.592 & 0.366 & 0.0728 \\
\hline Std. dev. & & 0.001 & 0.012 & 0.012 & 0.002 & 0.014 & 0.002 & 0.0008 \\
\hline
\end{tabular}

${ }^{\mathrm{a}}$ - means not measured. 
Table 13. Results of RWS with Advanced PC CWF Products

\begin{tabular}{|c|c|c|c|c|}
\hline Test No. & Mass CWF, g & $\begin{array}{c}\text { Volume Water, } \\
\mathrm{mL}\end{array}$ & $\mathrm{Cl}, \mathrm{mg} / \mathrm{L}$ & $N L(\mathrm{Cl}), \mathrm{g} / \mathrm{m}^{2}$ \\
\hline \multicolumn{5}{|c|}{ 20-g Products } \\
\hline PC02604 & 3.94 & 40.24 & 294 & 2.74 \\
\hline PC02605 & 2.22 & 22.19 & 335 & 3.05 \\
\hline PC02605-2 & 2.58 & 26.03 & 339 & 3.12 \\
\hline PC02606 & 2.34 & 26.65 & 273 & 2.83 \\
\hline PC02606-2 & 2.88 & 29.21 & 295 & 2.73 \\
\hline \multicolumn{5}{|c|}{ 500-g Products } \\
\hline PC02601 & 1.35 & 14.48 & 296 & 2.89 \\
\hline PC 03301-1 & 2.21 & 22.10 & 357.0 & 3.25 \\
\hline PC 03301-2 & 2.19 & 22.31 & 353.9 & 3.29 \\
\hline PC 03301-3 & 2.28 & 22.99 & 332.0 & 3.05 \\
\hline \multicolumn{5}{|c|}{ 26-kg Products } \\
\hline PC02701-2 & 3.95 & 39.72 & 335 & 3.07 \\
\hline $\mathrm{PC} 02701-11^{\mathrm{b}}$ & 2.29 & 26.02 & 271 & 2.81 \\
\hline PC02701-13 & 2.83 & 28.29 & 231 & 2.10 \\
\hline PC02701-15 & 1.48 & 14.82 & 423 & 3.86 \\
\hline
\end{tabular}

${ }^{\mathrm{a}}$ Sample taken from mid-radius of top half of product.

${ }^{\mathrm{b}}$ Sample taken from mid-radius of bottom half of product.

'Sample taken from center of bottom half of product.

${ }^{\mathrm{d}}$ Sample taken from edge of bottom half of product. 
Table 14. Results of 7-Day PCTs with Advanced PC CWF Materials, $N L(i)$ in $\mathrm{g} / \mathrm{m}^{2}$

\begin{tabular}{|c|c|c|c|c|c|c|c|c|}
\hline Test Number & $\mathrm{pH}$ & $\mathrm{Al}$ & B & $\mathrm{Cl}$ & Cs & $\mathrm{Li}$ & $\mathrm{Na}$ & Si \\
\hline \multicolumn{9}{|c|}{ Advanced PC CWF Product 02606} \\
\hline RWS & & 0.0039 & 0.0078 & 2.891 & 0.0219 & 0.019 & 0.759 & 0.0030 \\
\hline PCT-1 & 9.21 & 0.0807 & 0.0601 & 0.229 & 0.0427 & 0.462 & 0.126 & 0.0795 \\
\hline PCT-2 & 9.13 & 0.0810 & 0.0525 & 0.235 & 0.0408 & 0.450 & 0.127 & 0.0806 \\
\hline PCT-3 & 8.98 & 0.0774 & 0.0620 & 0.246 & 0.0396 & 0.444 & 0.124 & 0.0783 \\
\hline Mean total & & 0.0836 & 0.0660 & 3.13 & 0.0630 & 0.471 & 0.885 & 0.0824 \\
\hline \multicolumn{9}{|c|}{ Advanced PC CWF Product PC02701-2 } \\
\hline RWS & & 0.0046 & 0.0043 & 3.071 & 0.0090 & 0.024 & 0.890 & 0.0035 \\
\hline PCT-1 & 9.02 & 0.0805 & 0.0414 & 0.201 & 0.0224 & 0.470 & 0.121 & 0.0728 \\
\hline PCT-2 & 8.96 & 0.0673 & 0.0424 & 0.190 & 0.0264 & 0.418 & 0.108 & 0.0633 \\
\hline PCT-3 & 8.93 & 0.0735 & 0.0513 & 0.285 & 0.0238 & 0.470 & 0.131 & 0.0738 \\
\hline Mean total & & 0.0784 & 0.0493 & 3.30 & 0.0332 & 0.476 & 1.011 & 0.0735 \\
\hline \multicolumn{9}{|c|}{ Advanced PC CWF Product PC02701-13 } \\
\hline RWS & & 0.0032 & 0.0030 & 2.106 & 0.0080 & 0.018 & 0.645 & 0.0026 \\
\hline PCT-1 & 9.20 & 0.0918 & 0.0300 & 0.176 & 0.0178 & 0.460 & 0.130 & 0.0858 \\
\hline PCT-2 & 9.20 & 0.0892 & 0.0340 & 0.167 & 0.0160 & 0.432 & 0.125 & 0.0800 \\
\hline Mean total & & 0.0937 & 0.0350 & 2.28 & 0.0249 & 0.464 & 0.773 & 0.0855 \\
\hline
\end{tabular}

${ }^{\mathrm{a}}$ Sample taken from mid-radius of top half of product.

${ }^{\mathrm{b}}$ Sample taken from center of bottom half of product. 
Table 15. Concentrations in RWS Solutions in Filtrate Solutions, in mg/L

\begin{tabular}{|c|c|c|c|c|c|c|c|c|c|}
\hline & \multicolumn{3}{|c|}{ PC02601-1 } & \multicolumn{3}{c|}{ PC02601-2 } & \multicolumn{3}{c|}{ PC02601-3 } \\
\hline & $450 \mathrm{~nm}$ & $20 \mathrm{~nm}$ & Ratio $^{\mathrm{a}}$ & $450 \mathrm{~nm}$ & $20 \mathrm{~nm}$ & Ratio & $450 \mathrm{~nm}$ & $20 \mathrm{~nm}$ & Ratio \\
\hline $\mathrm{Al}$ & 0.645 & 0.0415 & 15.5 & 0.687 & 0.0523 & 13.1 & 0.719 & 0.0712 & 10.1 \\
\hline $\mathrm{B}$ & 0.0768 & 0.0656 & 1.17 & 0.0817 & 0.0641 & 1.27 & 0.0920 & 0.0717 & 1.28 \\
\hline $\mathrm{Cl}$ & 396 & $--\mathrm{b}^{-}$ & & 361 & -- & & 367 & -- & \\
\hline $\mathrm{Cs}$ & 0.0324 & 0.0303 & 1.07 & 0.0321 & 0.0306 & 1.05 & 0.0348 & 0.0322 & 1.08 \\
\hline $\mathrm{K}$ & 0.994 & 0.933 & 1.07 & 0.975 & 0.940 & 1.04 & 1.030 & 0.941 & 1.09 \\
\hline $\mathrm{Li}$ & 0.161 & 0.144 & 1.12 & 0.153 & 0.144 & 1.06 & 0.163 & 0.142 & 1.15 \\
\hline $\mathrm{I}$ & 0.579 & 0.617 & 0.94 & 0.595 & 0.590 & 1.01 & 0.602 & 0.616 & 0.98 \\
\hline $\mathrm{Na}$ & 200 & 196 & 1.02 & 195 & 194 & 1.01 & 197 & 197 & 1.00 \\
\hline $\mathrm{Si}$ & 0.671 & 0.0679 & 9.88 & 0.709 & 0.0769 & 9.22 & 0.801 & 0.0923 & 8.68 \\
\hline
\end{tabular}

${ }^{\mathrm{a}}$ Concentration in 450-nm filtrate/concentration in 20-nm filtrate.

b -- means not measured. 
Table 16. Results of RWS Tests with Developmental PC CWF Materials

\begin{tabular}{|c|c|c|c|c|c|}
\hline Test Number & $\begin{array}{c}\text { Hold Time, } \\
\mathrm{h}\end{array}$ & $\begin{array}{c}\text { Mass CWF, } \\
\mathrm{g}\end{array}$ & $\begin{array}{c}\text { Volume Water, } \\
\mathrm{mL}\end{array}$ & $\mathrm{Cl}, \mathrm{mg} / \mathrm{L}$ & $\mathrm{NL}(\mathrm{Cl}), \mathrm{g} / \mathrm{m}^{2}$ \\
\hline $4 \mathrm{~h}-1$ & 4 & 0.83 & 8.34 & 6.15 & 0.0563 \\
\hline $4 \mathrm{~h}-2$ & 4 & 0.86 & 8.60 & 6.68 & 0.0609 \\
\hline 4h-3 & 4 & 0.87 & 8.28 & 6.25 & 0.0542 \\
\hline Mean $\pm \mathrm{s}$ & & & & & $0.0570 \pm 0.0033$ \\
\hline $72 \mathrm{~h}-1$ & 72 & 0.99 & 10.41 & 362 & 3.47 \\
\hline $72 \mathrm{~h}-2$ & 72 & 1.02 & 10.28 & 395 & 3.63 \\
\hline $72 \mathrm{~h}-3$ & 72 & 1.01 & 10.25 & 380 & 3.52 \\
\hline Mean $\pm \mathrm{s}$ & & & & & $3.54 \pm 0.08$ \\
\hline $168 \mathrm{~h}-1$ & 168 & 0.88 & 8.85 & 705 & 6.46 \\
\hline $168 \mathrm{~h}-2$ & 168 & 1.14 & 11.20 & 747 & 6.69 \\
\hline $168 \mathrm{~h}-3$ & 168 & 0.95 & 9.97 & 697 & 6.67 \\
\hline Mean \pm s & & & & & $6.61 \pm 0.13$ \\
\hline
\end{tabular}


Table 17. XRD Results for Developmental PC CWF Materials

\begin{tabular}{|c|c|c|c|c|c|c|c|c|}
\hline \multicolumn{2}{|c|}{$\begin{array}{l}\text { Developmental } \\
\text { PC CWF } 4 \mathrm{~h}\end{array}$} & \multicolumn{2}{|c|}{$\begin{array}{l}\text { Developmental } \\
\text { PC CWF 72h }\end{array}$} & \multicolumn{2}{|c|}{$\begin{array}{l}\text { Developmental } \\
\text { PC CWF 168h }\end{array}$} & \multicolumn{3}{|c|}{$I D^{a}$} \\
\hline$d(\AA)$ & I (\%) & $\mathrm{d}(\AA)$ & I (\%) & $\mathrm{d}(\AA)$ & I (\%) & Phase & $\mathrm{d}(\AA)$ & I (\%) \\
\hline 6.346 & 27.3 & 6.321 & 24.9 & 6.319 & 30.3 & $\mathrm{~S}$ & 6.280 & 23 \\
\hline 4.469 & 6.4 & 4.453 & 5.4 & 4.456 & 4.0 & S & 4.440 & 4 \\
\hline \multirow[t]{2}{*}{4.203} & 1.4 & & & & & $\bar{N}$ & 4.165 & 58 \\
\hline & & & & 4.068 & 12.1 & $?$ & & \\
\hline 3.987 & 0.7 & & & & & $\dot{S}$ & 3.970 & 1 \\
\hline \multirow[t]{2}{*}{3.873} & 2.5 & & & & & $\mathrm{~N}$ & 3.834 & 78 \\
\hline & & & & 3.772 & 11.5 & $?$ & & \\
\hline \multirow[t]{2}{*}{3.641} & 100 & 3.633 & 100 & 3.632 & 100 & $S$ & 3.624 & 100 \\
\hline & & 3.230 & 13.5 & 3.224 & 71.6 & $\mathrm{H}$ & 3.260 & 11 \\
\hline \multirow[t]{2}{*}{3.023} & 2.4 & & & 3.015 & 2.1 & $\mathrm{~N}$ & 3.000 & 100 \\
\hline & & & & 2.942 & 4.2 & ? & & \\
\hline \multirow[t]{2}{*}{2.816} & 7.5 & 2.829 & 28.6 & 2.828 & 43.8 & $\mathrm{H}$ & 2.821 & 100 \\
\hline & & & & 2.678 & 2.4 & ? & & \\
\hline \multirow[t]{2}{*}{2.569} & 19.6 & 2.565 & 18.6 & 2.565 & 22.0 & S & 2.563 & 23 \\
\hline & & & & 2.529 & 11.2 & $?$ & & \\
\hline 2.377 & 20.8 & 2.375 & 19.9 & 2.375 & 22.0 & $\mathrm{~S}$ & 2.373 & 24 \\
\hline 2.095 & 29.2 & 2.074 & 31.0 & 2.093 & 33.3 & S & 2.093 & 35 \\
\hline 1.987 & 4.0 & 1.999 & 16.9 & 1.998 & 26.2 & $\mathrm{H}$ & 1.994 & 78 \\
\hline 1.894 & 3.2 & 1.893 & 4.6 & 1.893 & 3.8 & S & 1.873 & 6 \\
\hline \multirow[t]{2}{*}{1.813} & 3.4 & 1.812 & 2.1 & 1.812 & 2.9 & S & 1.812 & 4 \\
\hline & & & & 1.778 & 1.3 & $S$ & 1.775 & 2 \\
\hline \multirow[t]{2}{*}{1.742} & 2.9 & 1.741 & 3.1 & 1.742 & 3.9 & $S$ & 1.741 & 4 \\
\hline & & 1.631 & 6.5 & 1.630 & 8.2 & $\mathrm{H}$ & 1.628 & 26 \\
\hline 1.621 & 2.7 & 1.621 & 7.7 & 1.621 & 8.2 & S & 1.621 & 4 \\
\hline 1.569 & 9.8 & 1.569 & 11.9 & 1.569 & 13.0 & $S$ & 1.569 & 12 \\
\hline 1.522 & 4.9 & 1.522 & 5.5 & 1.522 & 4.7 & $\mathrm{~S}$ & 1.522 & 7 \\
\hline 1.479 & 7.4 & 1.480 & 10.4 & 1.479 & 12.0 & S & 1.479 & 10 \\
\hline
\end{tabular}

${ }^{\mathrm{a}}$ Identification by comparison with reference materials (see Table 6): $\mathrm{S}=$ Sodalite, $\mathrm{N}=$ Nepheline, $\mathrm{H}=$ Halite; ? = unknown.

${ }^{\mathrm{b}}$ Advanced PC CWF Product 02701.

${ }^{\mathrm{c}}$ Glass Loading PC CWF with 20 mass \% glass. 
Table 18. Mean $N L(i)$ for CWF Products in Replicate 7-Day PCTs, in $\mathrm{g} / \mathrm{m}^{2}$

\begin{tabular}{|c|c|c|c|c|c|c|c|c|c|}
\hline CWF Material & $\begin{array}{c}\text { Glass } \\
\text { Content }\end{array}$ & $\begin{array}{c}\text { Process } \\
\text { Temp. }\end{array}$ & $\mathrm{Al}$ & B & $\mathrm{Cl}$ & Cs & $\mathrm{Li}$ & $\mathrm{Na}$ & Si \\
\hline 5 kg baseline PC CWF & $50 \%$ & $850^{\circ} \mathrm{C}$ & 0.094 & 0.67 & 0.60 & 0.025 & 0.70 & 0.28 & 0.076 \\
\hline DTD baseline PC CWF & $50 \%$ & $850^{\circ} \mathrm{C}$ & 0.11 & 0.46 & 1.02 & 0.027 & 0.69 & 0.36 & 0.073 \\
\hline $25 \%$ glass loading PC CWF & $25 \%$ & $850^{\circ} \mathrm{C}$ & 0.10 & 0.20 & 0.62 & 0.063 & 0.47 & 0.23 & 0.079 \\
\hline Advanced PC CWF & $25 \%$ & $915^{\circ} \mathrm{C}$ & 0.085 & 0.050 & 2.91 & 0.040 & 0.47 & 0.89 & 0.081 \\
\hline HIP CWF & $25 \%$ & $850^{\circ} \mathrm{C}$ & 0.029 & 0.10 & 1.2 & $--^{a}$ & 0.32 & 0.28 & 0.034 \\
\hline Pu-doped HIP CWF & $25 \%$ & $850^{\circ} \mathrm{C}$ & 0.11 & 0.24 & 0.57 & -- & 0.50 & 0.27 & 0.10 \\
\hline Pu-doped PC CWF & $25 \%$ & $875^{\circ} \mathrm{C}$ & 0.082 & 0.086 & 3.8 & -- & 0.62 & 0.94 & 0.11 \\
\hline
\end{tabular}

a -- means not determined.

${ }^{\mathrm{b}}$ From Ref. [2]. 
Table 19. Results of PCT with Advanced PC CWF in Inter-laboratory Study

\begin{tabular}{|c|c|c|c|c|c|}
\hline Data Set & $\mathrm{pH}$ & {$[\mathrm{Al}], \mathrm{mg} / \mathrm{L}$} & {$[\mathrm{B}], \mathrm{mg} / \mathrm{L}$} & {$[\mathrm{Na}], \mathrm{mg} / \mathrm{L}$} & {$[\mathrm{Si}], \mathrm{mg} / \mathrm{L}$} \\
\hline $\mathrm{A}$ & $8.96 \pm 0.02$ & $24.8 \pm 0.4$ & $2.12 \pm 0.04$ & $31.8 \pm 0.6$ & $33.3 \pm 0.6$ \\
\hline $\mathrm{B}$ & $9.21 \pm 0.06$ & $24.5 \pm 1.7$ & $1.81 \pm 0.07$ & $27.6 \pm 1.0$ & $31.9 \pm 1.5$ \\
\hline $\mathrm{C}$ & $8.92 \pm 0.08$ & $23.2 \pm 1.1$ & $1.99 \pm 0.17$ & $32.7 \pm 1.2$ & $32.0 \pm 1.4$ \\
\hline $\mathrm{D}^{\mathrm{a}}$ & $9.03 \pm 0.06$ & $24.2 \pm 1.0$ & $2.52 \pm 0.18$ & $35.2 \pm 2.1$ & $34.1 \pm 1.2$ \\
\hline $\mathrm{E}^{\mathrm{a}}$ & $8.82 \pm 0.07$ & $22.1 \pm 0.4$ & $2.47 \pm 0.09$ & $31.4 \pm 0.2$ & $32.2 \pm 0.7$ \\
\hline $\mathrm{F}$ & $8.93 \pm 0.06$ & $23.3 \pm 0.6$ & $2.70 \pm 0.10$ & $31.3 \pm 0.6$ & $35.0 \pm 0.0$ \\
\hline $\mathrm{G}$ & Not reported & $11.7 \pm 0.6$ & $2.40 \pm 0.17$ & $27.3 \pm 7.5$ & $27.7 \pm 0.6$ \\
\hline $\mathrm{H}$ & $9.02 \pm 0.01$ & $25.8 \pm 0.5$ & $2.49 \pm 0.02$ & $30.5 \pm 0.3$ & $35.7 \pm 0.7$ \\
\hline
\end{tabular}

${ }^{\mathrm{a}}$ Extra set of triplicate tests conducted by Participant A (excluded from statistics). 
Table 20. Statistics for PCT with Advanced PC CWF in Inter-laboratory Study ${ }^{a}$

\begin{tabular}{|c|c|c|c|c|c|c|}
\hline & $x$ & $s_{x}$ & $s_{r}$ & $s_{R}$ & $I(r)$ & $I(R)$ \\
\hline $\mathrm{pH}$ & 9.01 & 0.117 & 0.050 & 0.124 & 0.142 & 0.351 \\
\hline $\mathrm{Al}, \mathrm{mg} / \mathrm{L}$ & 24.3 & 1.09 & 0.992 & 1.36 & 2.81 & 3.84 \\
\hline $\mathrm{B}, \mathrm{mg} / \mathrm{L}$ & 2.25 & 0.334 & 0.113 & 0.347 & 0.320 & 0.982 \\
\hline $\mathrm{Na}, \mathrm{mg} / \mathrm{L}$ & 30.2 & 2.23 & 3.15 & 3.40 & 8.91 & 9.62 \\
\hline $\mathrm{Si}, \mathrm{mg} / \mathrm{L}$ & 32.6 & 2.86 & 0.949 & 2.97 & 2.69 & 8.40 \\
\hline
\end{tabular}

${ }^{\mathrm{a}}$ Concentrations of $\mathrm{Al}, \mathrm{B}, \mathrm{Na}$, and $\mathrm{Si}$ in $\mathrm{mg} / \mathrm{L}$.

$x_{p}=$ response measured by participant $p$.

$p=$ number of participants.

$n=$ number of replicate tests $(=3)$.

$\bar{x}=$ consensus mean response.

$\bar{x}_{p}$ is the average of values by a participant in replicate tests: $\bar{x}_{p}=\Sigma x_{p} / n$.

$s_{x}$ is the standard deviation of the averages for replicate tests by the same participant from the

consensus average: $s_{x}=\left[\Sigma\left(\bar{x}_{p}-\bar{x}\right)^{2} /(p-1)\right]^{1 / 2}$.

$s_{r}$ is the pooled intra-laboratory standard deviation: $\left.s_{r}=\left[\Sigma s_{p}{ }^{2} / p\right)\right]^{1 / 2}$.

$s_{R}$ is the inter-laboratory estimate of precision: $s_{R}=\left(s_{x}^{2}+s_{r}^{2}(n-1) / n\right)^{1 / 2}$.

$I(r)$ is the estimated $95 \%$ repeatability level: $I(r)=2.83 \cdot s_{r}$.

$I(R)$ is the estimated $95 \%$ reproducibility level: $I(R)=2.83 \cdot s_{R}$. 
Table 21. Statistics for PCTs with Advanced PC CWF and with Borosilicate Glasses ${ }^{a}$

\begin{tabular}{|c|c|c|c|c|c|}
\hline Material & $p / n^{b}$ & $\mathrm{x}$ & $s_{x}$ & $s_{r}^{c}$ & $s_{R}{ }^{d}$ \\
\hline \multicolumn{6}{|c|}{ Aluminum } \\
\hline CWF & $6 / 3$ & 24.3 & 1.09 & 0.992 & 1.36 \\
\hline $\mathrm{LRM}^{\mathrm{e}}$ & $8 / 3$ & 14.3 & 2.42 & 0.922 & 2.59 \\
\hline SRL-G $(3 \times 1)^{\dagger}$ & $6 / 3$ & 3.84 & 0.256 & 0.124 & 0.275 \\
\hline SRL-G $(1 \times 3)^{g}$ & $6 / 3$ & 3.87 & $--^{\prime}$ & 0.250 & 0.343 \\
\hline SRL-P $P^{g}$ & $6 / 3$ & 3.51 & -- & 0.142 & 0.288 \\
\hline SRM $623^{g}$ & $6 / 3$ & 3.34 & -- & 0.200 & 0.228 \\
\hline ARM-1 ${ }^{9}$ & $6 / 3$ & 4.65 & -- & 0.269 & 0.468 \\
\hline Standard $^{n}$ & $6 / 3$ & 4.09 & -- & 0.095 & -- \\
\hline \multicolumn{6}{|c|}{ Boron } \\
\hline CWF & $6 / 3$ & 2.25 & 0.334 & 0.113 & 0.347 \\
\hline LRM & $8 / 3$ & 26.7 & 2.48 & 0.647 & 2.54 \\
\hline SRL-G $(3 \times 1)$ & $6 / 3$ & 14.7 & 0.523 & 0.287 & 0.573 \\
\hline SRL-G $(1 \times 3)$ & $6 / 3$ & 14.4 & -- & 0.465 & 0.724 \\
\hline SRL-P & $6 / 3$ & 25.3 & -- & 0.667 & 1.27 \\
\hline SRM 623 & $6 / 3$ & 7.05 & -- & 0.496 & 0.609 \\
\hline ARM-1 & $6 / 3$ & 27.5 & -- & 2.07 & 3.33 \\
\hline Standard & $6 / 3$ & 19.6 & -- & 0.524 & -- \\
\hline \multicolumn{6}{|c|}{ Sodium } \\
\hline CWF & $6 / 3$ & 30.2 & 2.23 & 3.15 & 3.40 \\
\hline LRM & $8 / 3$ & 160 & 11.5 & 4.06 & 11.9 \\
\hline SRL-G $(3 \times 1)$ & $6 / 3$ & 49.2 & 2.50 & 0.993 & 2.63 \\
\hline SRL-G $(1 \times 3)$ & $6 / 3$ & 49.9 & -- & 1.51 & 2.49 \\
\hline SRL-P & $6 / 3$ & 69.6 & -- & 3.47 & 3.90 \\
\hline SRM 623 & $6 / 3$ & 12.7 & -- & 0.87 & 0.961 \\
\hline ARM-1 & $6 / 3$ & 55.6 & -- & 4.16 & 5.08 \\
\hline Standard & $6 / 3$ & 39.3 & -- & 0.557 & -- \\
\hline \multicolumn{6}{|c|}{ Silicon } \\
\hline CWF & $6 / 3$ & 32.6 & 2.86 & 0.949 & 2.97 \\
\hline LRM & $8 / 3$ & 82.0 & 4.36 & 1.25 & 4.48 \\
\hline SRL-G $(3 \times 1)$ & $6 / 3$ & 110 & 4.30 & 1.09 & 4.39 \\
\hline SRL-G $(1 \times 3)$ & $6 / 3$ & 112 & -- & 3.10 & 4.18 \\
\hline SRL-P & $6 / 3$ & 110 & -- & 3.19 & 3.76 \\
\hline SRM 623 & $6 / 3$ & 46.1 & -- & 3.72 & 4.47 \\
\hline ARM-1 & $6 / 3$ & 80.2 & -- & 3.92 & 6.03 \\
\hline Standard & $6 / 3$ & 40.8 & -- & 0.897 & -- \\
\hline
\end{tabular}

${ }^{a}$ Concentrations of $\mathrm{Al}, \mathrm{B}, \mathrm{Na}$, and $\mathrm{Si}$ in $\mathrm{mg} / \mathrm{L}$.

${ }^{\mathrm{b}} p=$ number of participants or independent data sets; $n=$ number of replicate tests.

${ }^{\mathrm{c}} \%$ RDS repeatability $=100 s_{r} / \bar{x}$.

d $\%$ RDS reproducibility $=100 s_{R} / \bar{x}$.

${ }^{\mathrm{e}}$ Results from [10].

${ }^{\mathrm{f}}$ Results calculated from data in [11] for triplicate test conducted during week 1.

${ }^{\mathrm{g}}$ Results calculated from data in [11] for single tests run during weeks 1, 2, and 3 (from $s_{R}$ from Table C.2 and $s_{r}$ from Table C.3).

${ }^{\mathrm{h}}$ Results calculated from data in [10] for replicate analysis of a standard solution. (from Table C.7

"without* values").

i -- means not reported. 
Table 22. Results of Long-Term PCT with Baseline PC CWF

\begin{tabular}{|c|c|c|c|c|c|c|c|c|c|c|c|c|c|c|c|c|c|}
\hline $\begin{array}{l}\text { Test } \\
\text { Number }\end{array}$ & $\begin{array}{c}\text { Time, } \\
\mathrm{h}\end{array}$ & $\begin{array}{c}\mathrm{Al}, \\
\mathrm{mg} / \mathrm{L}\end{array}$ & $\begin{array}{l}N L(\mathrm{Al}), \\
\mathrm{g} / \mathrm{m}^{2}\end{array}$ & $\begin{array}{c}\mathrm{B}, \\
\mathrm{mg} / \mathrm{L}\end{array}$ & $\begin{array}{l}N L(B) \\
g / m^{2}\end{array}$ & $\begin{array}{c}\mathrm{Cl}, \\
\mathrm{mg} / \mathrm{L}\end{array}$ & $\begin{array}{c}N L(\mathrm{Cl}) \\
\mathrm{g} / \mathrm{m}^{2}\end{array}$ & $\begin{array}{l}\mathrm{Cs}, \\
\mathrm{mg} / \mathrm{L}\end{array}$ & $\begin{array}{c}N L(\mathrm{Cs}) \\
\mathrm{g} / \mathrm{m}^{2}\end{array}$ & $\begin{array}{c}\mathrm{I}, \\
\mathrm{mg} / \mathrm{L}\end{array}$ & $\begin{array}{l}N L(\mathrm{I}) \\
\mathrm{g} / \mathrm{m}^{2}\end{array}$ & $\begin{array}{c}\mathrm{Li}, \\
\mathrm{mg} / \mathrm{L}\end{array}$ & $\begin{array}{c}N L(\mathrm{Li}) \\
\mathrm{g} / \mathrm{m}^{2}\end{array}$ & $\begin{array}{l}\mathrm{Na}, \\
\mathrm{mg} / \mathrm{L}\end{array}$ & $\begin{array}{c}N L(\mathrm{Na}) \\
\mathrm{g} / \mathrm{m}^{2}\end{array}$ & $\begin{array}{c}\mathrm{Si}, \\
\mathrm{mg} / \mathrm{L}\end{array}$ & $\begin{array}{c}N L(\mathrm{Si}) \\
\mathrm{g} / \mathrm{m}^{2}\end{array}$ \\
\hline PPC-28-1 & 28 & 19.7 & 0.0814 & 88.9 & 1.27 & 23.5 & 0.318 & 0.0578 & 0.0254 & $--^{a}$ & -- & 8.41 & 1.23 & 71.6 & 0.330 & 44.1 & 0.0821 \\
\hline PPC-28-2 & 28 & 19.1 & 0.0777 & 90.6 & 1.28 & 23.3 & 0.309 & 0.0562 & 0.0243 & -- & -- & 8.46 & 1.21 & 70.9 & 0.321 & 42.8 & 0.0782 \\
\hline PPC-56-1 & 56 & 19.0 & 0.0768 & 119 & 1.68 & 25.9 & 0.342 & 0.0853 & 0.0368 & 0.043 & 0.282 & 10.7 & 1.53 & 81.6 & 0.368 & 46.0 & 0.0837 \\
\hline PPC-56-2 & 56 & 16.8 & 0.0682 & 117 & 1.66 & 24.4 & 0.325 & 0.0816 & 0.0354 & 0.0375 & 0.248 & 10.5 & 1.51 & 77.9 & 0.353 & 45.4 & 0.0830 \\
\hline PPC-89-1 & 89 & 18.9 & 0.0769 & 158 & 2.23 & 29.5 & 0.392 & 0.106 & 0.0461 & 0.0374 & 0.247 & 13.0 & 1.86 & 92.5 & 0.419 & 52.6 & 0.0963 \\
\hline PPC-89-2 & 89 & 19.8 & 0.0826 & 162 & 2.34 & 28.3 & 0.385 & 0.105 & 0.0464 & 0.0333 & 0.225 & 13.3 & 1.95 & 93.0 & 0.431 & 52.8 & 0.0989 \\
\hline PPC-89-3 & 89 & 17.3 & 0.0713 & 156 & 2.23 & 26.7 & 0.359 & 0.101 & 0.0441 & 0.0305 & 0.204 & 12.5 & 1.81 & 91.1 & 0.417 & 54.4 & 0.1007 \\
\hline PPC-89-4 & 89 & 18.6 & 0.0757 & 158 & 2.23 & 30.8 & 0.409 & -- & -- & 0.0339 & 0.224 & 13.1 & 1.88 & 90.6 & 0.410 & 51.6 & 0.0943 \\
\hline PPC-89-5 & 89 & 18.3 & 0.0732 & 159 & 2.20 & 33.4 & 0.436 & -- & -- & 0.0298 & 0.193 & 12.7 & 1.79 & 90.9 & 0.404 & 51.3 & 0.0921 \\
\hline PPC-89-6 & 89 & 17.9 & 0.0732 & 159 & 2.25 & 30.7 & 0.410 & -- & -- & 0.123 & 0.816 & 12.7 & 1.83 & 89.9 & 0.409 & 53.0 & 0.0974 \\
\hline
\end{tabular}

a -- means not determined. 
Table 23. Test Matrix for Long-Term PCTs with PC CWF and HIP CWF

\begin{tabular}{|c|c|c|c|c|c|}
\hline Test Number & Duration, $d$ & Solid/Water & Test Number & Duration, $d$ & Solid/Water \\
\hline \multicolumn{3}{|c|}{ Tests with PC CWF at $2300 \mathrm{~m}^{-1}$} & \multicolumn{3}{|c|}{ Tests with PC CWF at $23,000 \mathrm{~m}^{-1}$} \\
\hline LTPPCD-28 & 28 & $1 / 10$ & hLTPPCD-28 & 28 & $1 / 1$ \\
\hline LTPPCD-91-1 & 91 & $1 / 10$ & hLTPPCD-91-1 & 91 & $1 / 1$ \\
\hline LTPPCD-91-2 & 91 & $1 / 10$ & hLTPPCD-91-2 & 91 & $1 / 1$ \\
\hline LTPPCD-182-1 & 190 & $1 / 10$ & hLTPPCD-182-1 & 190 & $1 / 1$ \\
\hline LTPPCD-182-2 & 190 & $1 / 10$ & hLTPPCD-182-2 & 190 & $1 / 1$ \\
\hline LTPPCD-364-1 & 364 & $1 / 10$ & hLTPPCD-364-1 & 364 & $1 / 1$ \\
\hline LTPPCD-364-2 & 364 & $1 / 10$ & hLTPPCD-364-2 & 364 & $1 / 1$ \\
\hline \multicolumn{3}{|c|}{ Tests with HIP CWF at $2300 \mathrm{~m}^{-1}$} & \multicolumn{3}{|c|}{ Tests with HIP CWF at $23,000 \mathrm{~m}^{-1}$} \\
\hline PRD-28-1 & 28 & $1 / 10$ & hPRD-28 & 28 & $1 / 1$ \\
\hline PRD-28-2 & 28 & $1 / 10$ & & & \\
\hline PRD-91-1 & 91 & $1 / 10$ & hPRD-91 & 91 & $1 / 1$ \\
\hline PRD-91-2 & 91 & $1 / 10$ & & & \\
\hline PRD-182-1 & 182 & $1 / 10$ & hPRD-182 & 182 & $1 / 1$ \\
\hline PRD-182-2 & 182 & $1 / 10$ & & & \\
\hline PRD-364-1 & 364 & $1 / 10$ & hPRD364 & 364 & $1 / 1$ \\
\hline PRD-364-2 & 364 & $1 / 10$ & & & \\
\hline \multicolumn{6}{|c|}{ Tests with Baseline PC CWF at $2300 \mathrm{~m}^{-1}$} \\
\hline PPC-28-1 & 28 & $1 / 10$ & & & \\
\hline PPC-28-2 & 28 & $1 / 10$ & & & \\
\hline PPC-56-1 & 56 & $1 / 10$ & & & \\
\hline PPC-56-2 & 56 & $1 / 10$ & & & \\
\hline PPC-89-1 & 89 & $1 / 10$ & & & \\
\hline PPC-89-2 & 89 & $1 / 10$ & & & \\
\hline PPC-89-3 & 89 & $1 / 10$ & & & \\
\hline PPC-89-4 & 89 & $1 / 10$ & & & \\
\hline PPC-89-5 & 89 & $1 / 10$ & & & \\
\hline PPC-89-6 & 89 & $1 / 10$ & & & \\
\hline
\end{tabular}


Table 24. Solution Concentrations in Long-Term PCTs with PC CWF and HIP CWF

\begin{tabular}{|c|c|c|c|c|c|c|c|}
\hline \multirow[b]{2}{*}{ Test No. } & \multirow[b]{2}{*}{ Time, d } & \multicolumn{6}{|c|}{$C(i), \mathrm{mg} / \mathrm{L}$} \\
\hline & & $\mathrm{Al}$ & $\mathrm{B}$ & $\mathrm{Cl}$ & $\mathrm{Li}$ & $\mathrm{Na}$ & Si \\
\hline \multicolumn{8}{|c|}{ Tests with PC CWF at $2300 \mathrm{~m}^{-1}$} \\
\hline LTPPCD-28 & 28 & 27.8 & 3.08 & 34.7 & 7.88 & 30.0 & 39.6 \\
\hline LTPPCD-91-1 & 91 & 29.9 & 4.20 & 42.5 & 8.22 & 25.9 & 44.7 \\
\hline LTPPCD-91-2 & 91 & 30.0 & 3.82 & 39.7 & 8.52 & 27.6 & 45.2 \\
\hline LTPPCD-182-1 & 182 & 34.8 & 5.75 & 35.6 & 9.69 & 25.8 & 50.0 \\
\hline LTPPCD-182-2 & 182 & 31.6 & 6.23 & 35.6 & 10.24 & 25.8 & 46.6 \\
\hline LTPPCD-364-1 & 364 & 34.9 & 6.16 & 34.6 & 13.00 & 24.7 & 57.0 \\
\hline LTPPCD-364-2 & 364 & 34.7 & 6.08 & 37.1 & 12.89 & 24.0 & 55.6 \\
\hline \multicolumn{8}{|c|}{ Tests with PC CWF at $23,000 \mathrm{~m}^{-1}$} \\
\hline hLTPPCD-28 & 28 & 39.5 & 12.6 & 313 & 51.0 & 128 & 39.4 \\
\hline hLTPPCD-91-1 & 91 & 34.6 & 15.9 & 321 & 52.2 & 101 & 38.2 \\
\hline hLTPPCD-91-2 & 91 & 35.5 & 16.3 & 314 & 52.4 & 96.5 & 39.4 \\
\hline hLTPPCD-182-1 & 182 & 37.2 & 27.1 & 314 & 57.4 & 94.3 & 40.0 \\
\hline hLTPPCD-182-2 & 182 & 37.9 & 29.6 & 306 & 57.6 & 98.8 & 42.6 \\
\hline hLTPPCD-364-1 & 364 & 34.6 & 30.4 & 283 & 67.1 & 83 & 50.0 \\
\hline hLTPPCD-364-2 & 364 & 30.4 & 28.9 & 291 & 67.0 & 80 & 41.9 \\
\hline \multicolumn{8}{|c|}{ Tests with HIP CWF at $2300 \mathrm{~m}^{-1}$} \\
\hline PRD-28-1 & 28 & 9.70 & 13.2 & 133 & 5.52 & 75.8 & 22.8 \\
\hline PRD-28-2 & 28 & 10.7 & 13.6 & 131 & 6.32 & 75.2 & 23.2 \\
\hline PRD-91-1 & 91 & 10.3 & 29.9 & 140 & 8.20 & 96.6 & 28.2 \\
\hline PRD-91-2 & 91 & 11.5 & 29.9 & 137 & 8.32 & 94.7 & 28.0 \\
\hline PRD-182-1 & 182 & 5.75 & 38.1 & 133 & 8.19 & 92.0 & 17.5 \\
\hline PRD-182-2 & 182 & 5.59 & 37.4 & 139 & 7.65 & 89.0 & 17.0 \\
\hline PRD-364-1 & 364 & 8.27 & 40.7 & 138 & 9.64 & 78.7 & 33.9 \\
\hline PRD-364-2 & 364 & 8.89 & 21.3 & 133 & 5.09 & 44.3 & 56.7 \\
\hline \multicolumn{8}{|c|}{ Tests with HIP CWF at $23,000 \mathrm{~m}^{-1}$} \\
\hline hPRD-28 & 28 & 3.89 & 72.3 & 1480 & 49.3 & 538 & 19.2 \\
\hline hPRD-91 & 91 & 3.24 & 224 & 1160 & 77.4 & 858 & 36.9 \\
\hline hPRD-182 & 182 & 1.55 & 347 & 1100 & 98.6 & 643 & 28.5 \\
\hline hPRD-364 & 364 & 0.930 & 328 & 1350 & 102 & 758 & 44.1 \\
\hline \multicolumn{8}{|c|}{ Tests with Baseline PC CWF at $2300 \mathrm{~m}^{-1}$} \\
\hline PPC-28-1 & 28 & 19.7 & 88.9 & 23.5 & 8.41 & 71.4 & 44.1 \\
\hline PPC-28-2 & 28 & 19.1 & 90.6 & 23.3 & 8.46 & 70.6 & 42.8 \\
\hline PPC-56-1 & 56 & 19.0 & 119 & 25.9 & 10.7 & 81.3 & 46.0 \\
\hline PPC-56-2 & 56 & 16.8 & 117 & 24.4 & 10.5 & 77.6 & 45.4 \\
\hline PPC-89-1 & 89 & 18.9 & 158 & 29.5 & 13.0 & 92.1 & 52.6 \\
\hline PPC-89-2 & 89 & 19.8 & 162 & 28.3 & 13.3 & 92.4 & 52.8 \\
\hline PPC-89-3 & 89 & 17.3 & 156 & 26.7 & 12.5 & 90.6 & 54.4 \\
\hline PPC-89-4 & 89 & 18.6 & 158 & 30.8 & 13.1 & 90.1 & 51.6 \\
\hline PPC-89-5 & 89 & 18.3 & 159 & 33.4 & 12.7 & 90.4 & 51.3 \\
\hline PPC-89-6 & 89 & 17.9 & 159 & 30.7 & 12.7 & 89.4 & 53.0 \\
\hline
\end{tabular}


Table 25. Results of Long-Term PCTs with PC CWF and HIP CWF, NL(i) in $\mathrm{g} / \mathrm{m}^{2}$

\begin{tabular}{|c|c|c|c|c|c|c|c|}
\hline \multirow[b]{2}{*}{ Test No. } & \multirow[b]{2}{*}{ Time, d } & \multicolumn{6}{|c|}{$N L(i), \mathrm{g} / \mathrm{m}^{2}$} \\
\hline & & $\mathrm{Al}$ & B & $\mathrm{Cl}$ & $\mathrm{Li}$ & $\mathrm{Na}$ & Si \\
\hline \multicolumn{8}{|c|}{ Tests with PC CWF at $2300 \mathrm{~m}^{-1}$} \\
\hline LTPPCD-28 & 28 & 0.102 & 0.096 & 0.319 & 0.842 & 0.104 & 0.091 \\
\hline LTPPCD-91-1 & 91 & 0.109 & 0.130 & 0.387 & 0.872 & 0.0894 & 0.102 \\
\hline LTPPCD-91-2 & 91 & 0.109 & 0.119 & 0.362 & 0.903 & 0.0954 & 0.104 \\
\hline LTPPCD-182-1 & 182 & 0.127 & 0.179 & 0.324 & 1.03 & 0.0891 & 0.115 \\
\hline LTPPCD-182-2 & 182 & 0.115 & 0.194 & 0.325 & 1.09 & 0.0894 & 0.107 \\
\hline LTPPCD-364-1 & 364 & 0.126 & 0.191 & 0.315 & 1.38 & 0.0851 & 0.130 \\
\hline LTPPCD-364-2 & 364 & 0.126 & 0.189 & 0.338 & 1.37 & 0.0828 & 0.127 \\
\hline \multicolumn{8}{|c|}{ Tests with PC CWF at $23,000 \mathrm{~m}^{-1}$} \\
\hline hLTPPCD-28 & 28 & 0.0143 & 0.0390 & 0.285 & 0.540 & 0.0440 & 0.00901 \\
\hline hLTPPCD-91-1 & 91 & 0.0126 & 0.0497 & 0.294 & 0.556 & 0.0349 & 0.00878 \\
\hline hLTPPCD-91-2 & 91 & 0.0129 & 0.0505 & 0.285 & 0.554 & 0.0333 & 0.00902 \\
\hline hLTPPCD-182-1 & 182 & 0.0135 & 0.0839 & 0.286 & 0.607 & 0.0325 & 0.00914 \\
\hline hLTPPCD-182-2 & 182 & 0.0137 & 0.0919 & 0.279 & 0.611 & 0.0341 & 0.00976 \\
\hline hLTPPCD-364-1 & 364 & 0.0127 & 0.0952 & 0.260 & 0.718 & 0.0289 & 0.01155 \\
\hline hLTPPCD-364-2 & 364 & 0.0110 & 0.0897 & 0.265 & 0.709 & 0.0275 & 0.00957 \\
\hline \multicolumn{8}{|c|}{ Tests with HIP CWF at $2300 \mathrm{~m}^{-1}$} \\
\hline PRD-28-1 & 28 & 0.033 & 0.384 & 1.21 & 0.544 & 0.285 & 0.051 \\
\hline PRD-28-2 & 28 & 0.037 & 0.394 & 1.19 & 0.625 & 0.285 & 0.054 \\
\hline PRD-91-1 & 91 & 0.036 & 0.838 & 1.23 & 0.783 & 0.352 & 0.062 \\
\hline PRD-91-2 & 91 & 0.039 & 0.865 & 1.24 & 0.816 & 0.354 & 0.063 \\
\hline PRD-182-1 & 182 & 0.021 & 1.101 & 1.21 & 0.802 & 0.0344 & 0.041 \\
\hline PRD-182-2 & 182 & 0.021 & 1.083 & 1.26 & 0.752 & 0.335 & 0.040 \\
\hline PRD-364-1 & 364 & 0.034 & 1.173 & 1.25 & 0.944 & 0.298 & 0.081 \\
\hline PRD-364-2 & 364 & 0.045 & 0.618 & 1.20 & 0.508 & 0.179 & 0.094 \\
\hline \multicolumn{8}{|c|}{ Tests with HIP CWF at $23,000 \mathrm{~m}^{-1}$} \\
\hline hPRD-28 & 28 & 0.0015 & 0.205 & 1.31 & 0.473 & 0.197 & 0.0043 \\
\hline hPRD-91 & 91 & 0.0014 & 0.632 & 1.03 & 0.739 & 0.313 & 0.0082 \\
\hline hPRD-182 & 182 & 0.0026 & 1.06 & 1.05 & 1.02 & 0.254 & 0.0083 \\
\hline hPRD-364 & 364 & 0.0042 & 0.967 & 1.24 & 1.03 & 0.292 & 0.014 \\
\hline \multicolumn{8}{|c|}{ Tests with Baseline PC CWF at $2300 \mathrm{~m}^{-1}$} \\
\hline PPC-28-1 & 28 & 0.0814 & 1.27 & 0.318 & 1.23 & 0.330 & 0.0821 \\
\hline PPC-28-2 & 28 & 0.0777 & 1.28 & 0.309 & 1.21 & 0.321 & 0.0782 \\
\hline PPC-56-1 & 56 & 0.0768 & 1.68 & 0.342 & 1.53 & 0.368 & 0.0837 \\
\hline PPC-56-2 & 56 & 0.0682 & 1.66 & 0.325 & 1.51 & 0.353 & 0.0830 \\
\hline PPC-89-1 & 89 & 0.0769 & 2.23 & 0.392 & 1.86 & 0.419 & 0.0963 \\
\hline PPC-89-2 & 89 & 0.0826 & 2.34 & 0.385 & 1.95 & 0.431 & 0.0989 \\
\hline PPC-89-3 & 89 & 0.0713 & 2.23 & 0.359 & 1.81 & 0.417 & 0.1007 \\
\hline PPC-89-4 & 89 & 0.0757 & 2.23 & 0.409 & 1.88 & 0.410 & 0.0943 \\
\hline PPC-89-5 & 89 & 0.0732 & 2.20 & 0.436 & 1.79 & 0.404 & 0.0921 \\
\hline PPC-89-6 & 89 & 0.0732 & 2.25 & 0.410 & 1.83 & 0.409 & 0.0974 \\
\hline
\end{tabular}


Table 26. Test Matrix for Long-Term PCTs with Binder Glass

\begin{tabular}{|c|c|c|c|c|c|}
\hline Test Number & Duration, $d$ & Solid/Water & Test Number & Duration, $d$ & Solid/Water \\
\hline \multicolumn{3}{|c|}{ Tests with Binder Glass at $2300 \mathrm{~m}^{-1}$} & \multicolumn{3}{|c|}{ Tests with Binder Glass at $23,000 \mathrm{~m}^{-1}$} \\
\hline PGD-7-1 & 7 & $1 / 10$ & PGDH-7 & 7 & $1 / 1$ \\
\hline PGD-7-2 & 7 & $1 / 10$ & PGDH-28 & 28 & $1 / 1$ \\
\hline PGD-28 & 28 & $1 / 10$ & PGDH-91 & 91 & $1 / 1$ \\
\hline PGD-91 & 91 & $1 / 10$ & \multicolumn{3}{|c|}{ Tests with Binder Glass at $15,000 \mathrm{~m}^{-1}$} \\
\hline PGD-182 & 182 & $1 / 10$ & PGDH-183 & 182 & $1 / 1$ \\
\hline PBD-364 & 364 & $1 / 10$ & PBDH-364 & 364 & $1 / 1$ \\
\hline \multicolumn{3}{|c|}{ Tests with PC Glass at $2300 \mathrm{~m}^{-1}$} & \multicolumn{3}{|c|}{ Tests with PC Glass at $23,000 \mathrm{~m}^{-1}$} \\
\hline LTPGD-7 $^{\mathrm{a}}$ & 7 & $1 / 10$ & hLTPGD-7 & 7 & $1 / 1$ \\
\hline LTPGD-28 & 28 & $1 / 10$ & hLTPGD-28 & 28 & $1 / 1$ \\
\hline LTPGD-91-1 & 91 & $1 / 10$ & hLTPGD-91-1 & 91 & $1 / 1$ \\
\hline LTPGD-91-2 & 91 & $1 / 10$ & hLTPGD-91-2 & 91 & $1 / 1$ \\
\hline LTPGD-182-1 & 190 & $1 / 10$ & hLTPGD-182-1 & 190 & $1 / 1$ \\
\hline LTPGD-182-2 & 190 & $1 / 10$ & hLTPGD-182-2 & 190 & $1 / 1$ \\
\hline LTPGD-364-1 & 364 & $1 / 10$ & hLTPGD-364-1 & 364 & $1 / 1$ \\
\hline LTPGD-364-2 & 364 & $1 / 10$ & hLTPGD-364-2 & 364 & $1 / 1$ \\
\hline
\end{tabular}

${ }^{\mathrm{a}}$ Five replicate tests. 
Table 27. Solution Concentrations in Long-Term PCTs with Binder Glass, in mg/L

\begin{tabular}{|c|c|c|c|c|c|c|}
\hline & & \multicolumn{5}{|c|}{$C(i), \mathrm{mg} / \mathrm{L}$} \\
\hline Test No. & Time, d & $\mathrm{Al}$ & B & $\mathrm{Li}$ & $\mathrm{Na}$ & Si \\
\hline \multicolumn{7}{|c|}{ Tests with Binder Glass at $2300 \mathrm{~m}^{-1}$} \\
\hline PGD-7-1 & 7 & 1.56 & 238 & $--^{a}$ & 113 & 72.4 \\
\hline PGD-7-2 & 7 & 1.32 & 244 & -- & 114 & 67.6 \\
\hline PGD-28 & 28 & 0.904 & 411 & -- & 167 & 69.0 \\
\hline PGD-91 & 91 & 0.768 & 828 & -- & 410 & 81.8 \\
\hline PGD-182 & 182 & 0.865 & 1010 & -- & 467 & 66.7 \\
\hline PBD-364 & 364 & & 1460 & -- & & 73.8 \\
\hline \multicolumn{7}{|c|}{ Tests with Binder Glass at 23,000 $\mathrm{m}^{-1}$} \\
\hline PGDH-7 & 7 & 0.959 & 1570 & -- & 694 & 127 \\
\hline PGDH-28 & 28 & 0.930 & 2760 & -- & 1040 & 116 \\
\hline PGDH-91 & 91 & 1.070 & 5910 & -- & 1770 & 85.7 \\
\hline \multicolumn{7}{|c|}{ Tests with Binder Glass at $1500 \mathrm{~m}^{-1}$} \\
\hline PGDH-182 & 182 & 1.832 & 3610 & -- & 1520 & 93.7 \\
\hline PBDH-364 & 364 & & 4470 & -- & & 89.8 \\
\hline \multicolumn{7}{|c|}{ Tests with PC Glass at $2300 \mathrm{~m}^{-1}$} \\
\hline LTPGD-7 $^{\text {b }}$ & 7 & 1.12 & 450 & 0.3 & 239 & 75.6 \\
\hline LTPGD-28 & 28 & 0.85 & 731 & 0.0 & 380 & 71.7 \\
\hline LTPGD-91-1 & 91 & 0.63 & 1130 & 0.5 & 477 & 75.2 \\
\hline LTPGD-91-2 & 91 & 0.64 & 1230 & 0.5 & 488 & 72.4 \\
\hline LTPGD-182-1 & 182 & 0.81 & 1260 & 0.8 & 674 & 72.2 \\
\hline LTPGD-182-2 & 182 & 0.80 & 1260 & 0.8 & 655 & 77.8 \\
\hline LTPGD-364-1 & 364 & 0.59 & 1300 & 0.8 & 683.8 & 66.6 \\
\hline LTPGD-364-2 & 364 & 0.69 & 1260 & 0.7 & 666.4 & 74.8 \\
\hline \multicolumn{7}{|c|}{ Tests with PC Glass at $23,000 \mathrm{~m}^{-1}$} \\
\hline hLTPGD-7 & 7 & 0.446 & 2400 & 1.85 & 1160 & 110 \\
\hline hLTPGD-28 & 28 & 0.555 & 3840 & 0.13 & 1770 & 111 \\
\hline hLTPGD-91-1 & 91 & 0.743 & 6220 & 2.56 & 2300 & 118 \\
\hline hLTPGD-91-2 & 91 & 0.754 & 6230 & 2.33 & 2260 & 118 \\
\hline hLTPGD-182-1 & 182 & 1.10 & 6670 & 4.00 & 3000 & 118 \\
\hline hLTPGD-182-2 & 182 & 1.25 & 7330 & 3.97 & 3170 & 117 \\
\hline hLTPGD-364-1 & 364 & 1.21 & 7120 & 3.14 & 3310 & 113 \\
\hline hLTPGD-364-2 & 364 & 1.26 & 7070 & 3.40 & 3310 & 121 \\
\hline
\end{tabular}

${ }^{\mathrm{a}}$ Not analyzed.

${ }^{\mathrm{b}}$ Average values for 5 replicate tests. 
Table 28. Results of Long-Term PCTs with Binder Glass and PC Glass, $N L(i)$ in $\mathrm{g} / \mathrm{m}^{2}$

\begin{tabular}{|c|c|c|c|c|c|c|}
\hline & & \multicolumn{5}{|c|}{$N L(i), \mathrm{g} / \mathrm{m}^{2}$} \\
\hline Test No. & $\begin{array}{l}\text { Time, } \\
\text { d }\end{array}$ & $\mathrm{Al}$ & B & $\mathrm{Li}$ & $\mathrm{Na}$ & $\mathrm{Si}$ \\
\hline \multicolumn{7}{|c|}{ Tests with Binder Glass at $2300 \mathrm{~m}^{-1}$} \\
\hline PGD-7-1 & 7 & 0.016 & 1.67 & $--^{a}$ & 0.98 & 0.103 \\
\hline PGD-7-2 & 7 & 0.014 & 1.71 & -- & 0.99 & 0.096 \\
\hline PGD-28 & 28 & 0.009 & 2.79 & -- & 4.10 & 0.095 \\
\hline PGD-91 & 91 & 0.008 & 5.73 & -- & 3.52 & 0.115 \\
\hline PGD-182 & 182 & 0.009 & 6.90 & -- & 3.95 & 0.093 \\
\hline PBD-364 & 364 & & & -- & & \\
\hline \multicolumn{7}{|c|}{ Tests with Binder Glass at $23,000 \mathrm{~m}^{-1}$} \\
\hline PGDH-7 & 7 & 0.001 & 1.09 & -- & 0.60 & 0.018 \\
\hline PGDH-28 & 28 & 0.002 & 1.93 & -- & 0.91 & 0.017 \\
\hline PGDH-91 & 91 & 0.001 & 4.55 & -- & 1.69 & 0.013 \\
\hline \multicolumn{7}{|c|}{ Tests with Binder Glass at $1500 \mathrm{~m}^{-1}$} \\
\hline PGDH-182 & 182 & 0.007 & 3.76 & -- & 1.96 & 0.022 \\
\hline PBDH-364 & 364 & & & -- & -- & -- \\
\hline \multicolumn{7}{|c|}{ Tests with PC Glass at $2300 \mathrm{~m}^{-1}$} \\
\hline LTPGD- $^{\mathrm{b}}$ & 7 & 0.0153 & 3.50 & 3.72 & 2.00 & 0.119 \\
\hline LTPGD-28 & 28 & 0.0116 & 5.70 & -- & 3.19 & 0.113 \\
\hline LTPGD-91-1 & 91 & 0.00851 & 8.80 & 5.73 & 4.00 & 0.118 \\
\hline LTPGD-91-2 & 91 & 0.00862 & 9.58 & 5.89 & 4.09 & 0.113 \\
\hline LTPGD-182-1 & 182 & 0.0110 & 9.82 & 8.57 & 5.65 & 0.113 \\
\hline LTPGD-182-2 & 182 & 0.0109 & 9.82 & 8.85 & 5.49 & 0.122 \\
\hline LTPGD-364-1 & 364 & 0.00803 & 10.1 & 9.04 & 5.72 & 0.104 \\
\hline LTPGD-364-2 & 364 & 0.00935 & 9.76 & 7.80 & 5.58 & 0.117 \\
\hline \multicolumn{7}{|c|}{ Tests with PC Glass at $23,000 \mathrm{~m}^{-1}$} \\
\hline hLTPGD-7 & 7 & 0.000606 & 1.87 & 2.02 & 0.973 & 0.0173 \\
\hline hLTPGD-28 & 28 & 0.000756 & 3.00 & 0.141 & 1.49 & 0.0175 \\
\hline hLTPGD-91-1 & 91 & 0.00101 & 4.85 & 2.78 & 1.93 & 0.0185 \\
\hline hLTPGD-91-2 & 91 & 0.00102 & 4.85 & 2.53 & 1.89 & 0.0185 \\
\hline hLTPGD-182-1 & 182 & 0.00149 & 5.18 & 4.33 & 2.52 & 0.0184 \\
\hline hLTPGD-182-2 & 182 & 0.00170 & 5.70 & 4.30 & 2.65 & 0.0183 \\
\hline hLTPGD-364-1 & 364 & 0.00164 & 5.54 & 3.42 & 2.78 & 0.0177 \\
\hline hLTPGD-364-2 & 364 & 0.00171 & 5.51 & 3.70 & 2.78 & 0.0190 \\
\hline
\end{tabular}

${ }^{\mathrm{a}}$ Not analyzed.

${ }^{\mathrm{b}}$ Average values for 5 replicate tests. 
Table 29. Orthosilicic Acid Concentrations and $\mathrm{pH}$ from Long-Term PCTs with PC CWF and HIP CWF

\begin{tabular}{|c|c|c|c|c|c|}
\hline Test Number & $\begin{array}{c}{\left[\mathrm{H}_{4} \mathrm{SiO}_{4}\right]} \\
\mathrm{mg} / \mathrm{L}\end{array}$ & $\mathrm{pH}$ & Test Number & $\begin{array}{c}{\left[\mathrm{H}_{4} \mathrm{SiO}_{4}\right]} \\
\mathrm{mg} / \mathrm{L}\end{array}$ & $\mathrm{pH}$ \\
\hline \multicolumn{3}{|c|}{ Tests with PC CWF at $2300 \mathrm{~m}^{-1}$} & \multicolumn{3}{|c|}{ Tests with PC CWF at $23,000 \mathrm{~m}^{-1}$} \\
\hline LTPPCD-7 $^{\mathrm{a}}$ & 108 & 9.07 & hLTPPCD-7 & 111 & 9.45 \\
\hline LTPPCD-28 & 136 & 8.99 & hLTPPCD-28 & 135 & 9.23 \\
\hline LTPPCD-91-1 & 153 & 9.14 & hLTPPCD-91-1 & 131 & 9.19 \\
\hline LTPPCD-91-2 & 155 & 9.12 & hLTPPCD-91-2 & 135 & 9.27 \\
\hline LTPPCD-182-1 & 171 & 9.36 & hLTPPCD-182-1 & 137 & 9.46 \\
\hline LTPPCD-182-2 & 160 & 9.33 & hLTPPCD-182-2 & 146 & 9.50 \\
\hline LTPPCD-364-1 & 196 & 9.34 & hLTPPCD-364-1 & 171 & 9.52 \\
\hline LTPPCD-364-2 & 191 & 9.31 & hLTPPCD-364-2 & 144 & 9.52 \\
\hline \multicolumn{3}{|c|}{ Tests with HIP CWF at $2300 \mathrm{~m}^{-1}$} & \multicolumn{3}{|c|}{ Tests with HIP CWF at $23,000 \mathrm{~m}^{-1}$} \\
\hline PRD-7 $^{b}$ & 52.8 & 9.09 & hPRD-7 & 42.5 & 8.87 \\
\hline PRD-28 & 78.9 & 8.77 & hPRD-28 & 65.8 & 8.91 \\
\hline PRD-91-1 & 96.5 & 8.65 & hPRD-91 & 127 & 8.82 \\
\hline PRD-91-2 & 96.0 & 8.72 & & & \\
\hline PRD-182-1 & 60.0 & 8.62 & hPRD-182 & 97.7 & 8.76 \\
\hline PRD-182-2 & 58.3 & 8.63 & & & \\
\hline PRD-364-1 & 116 & 9.00 & hPRD-364 & 151 & 8.69 \\
\hline PRD-364-2 & 122 & 8.94 & & & \\
\hline \multicolumn{6}{|c|}{ Tests with Baseline PC CWF at $2300 \mathrm{~m}^{-1}$} \\
\hline PPC-7 ${ }^{\mathrm{C}}$ & $134 \pm 2$ & $8.75 \pm 0.08$ & & & \\
\hline PPC-28-1 & 151 & 8.60 & & & \\
\hline PPC-28-2 & 147 & 8.60 & & & \\
\hline PPC-56-1 & 158 & 8.47 & & & \\
\hline PPC-56-2 & 156 & 8.44 & & & \\
\hline PPC-89-1 & 180 & 8.79 & & & \\
\hline PPC-89-2 & 181 & 8.79 & & & \\
\hline PPC-89-3 & 187 & 8.78 & & & \\
\hline PPC-89-4 & 177 & 8.77 & & & \\
\hline PPC-89-5 & 176 & 8.77 & & & \\
\hline PPC-89-6 & 182 & 8.77 & & & \\
\hline
\end{tabular}


Table 30. Orthosilicic Acid Concentrations and $\mathrm{pH}$ from Long-Term PCTs with Binder Glass

\begin{tabular}{|c|c|c|c|c|c|}
\hline Test Number & $\begin{array}{c}{\left[\mathrm{H}_{4} \mathrm{SiO}_{4}\right]} \\
\mathrm{mg} / \mathrm{L}\end{array}$ & $\mathrm{pH}$ & Test Number & $\begin{array}{c}{\left[\mathrm{H}_{4} \mathrm{SiO}_{4}\right]} \\
\mathrm{mg} / \mathrm{L}\end{array}$ & $\mathrm{pH}$ \\
\hline \multicolumn{3}{|c|}{ Tests with Binder Glass at $2300 \mathrm{~m}^{-1}$} & \multicolumn{3}{|c|}{ Tests with Binder Glass at $23,000 \mathrm{~m}^{-1}$} \\
\hline PGD-7-1 & 248 & 8.53 & PGDH-7 & 436 & 8.42 \\
\hline PGD-7-1 & 232 & 8.49 & PGDH-28 & 398 & 8.26 \\
\hline PGD-28 & 237 & 8.65 & PGDH-91 & 294 & 7.75 \\
\hline PGD-91 & 280 & 8.28 & \multicolumn{3}{|c|}{ Tests with Binder Glass at $23,000 \mathrm{~m}^{-1}$} \\
\hline PGD-182 & 229 & 8.41 & PGDH-182 & 321 & 8.03 \\
\hline PBD-364 & 253 & 8.50 & PBDH-364 & 308 & 8.12 \\
\hline \multicolumn{3}{|c|}{ Tests with PC Glass at $2300 \mathrm{~m}^{-1}$} & \multicolumn{3}{|c|}{ Tests with PC Glass at $23,000 \mathrm{~m}^{-1}$} \\
\hline LTPGD-7 $^{\mathrm{a}}$ & 259 & 8.63 & hLTPGD-7 & 379 & 8.29 \\
\hline LTPGD-28 & 246 & 8.40 & hLTPGD-28 & 381 & 8.04 \\
\hline LTPGD-91-1 & 258 & 8.47 & hLTPGD-91-1 & 403 & 7.98 \\
\hline LTPGD-91-2 & 248 & 8.45 & hLTPGD-91-2 & 404 & 7.97 \\
\hline LTPGD-182-1 & 247 & 8.54 & hLTPGD-182-1 & 403 & 7.92 \\
\hline LTPGD-182-2 & 267 & 8.54 & hLTPGD-182-2 & 400 & 7.91 \\
\hline LTPGD-364-1 & 228 & 8.51 & hLTPGD-364-1 & 386 & 7.81 \\
\hline LTPGD-364-2 & 256 & 8.51 & hLTPGD-364-2 & 415 & 7.80 \\
\hline
\end{tabular}

${ }^{\mathrm{a} A v e r a g e ~ o f ~} 5$ replicate tests. 
Table 31. Processing Conditions for T-t PC CWF Products

\begin{tabular}{|c|c|c|c|c|c|c|}
\hline & \multicolumn{6}{|c|}{ Processing Temperature, ${ }^{\circ} \mathrm{C}$} \\
\hline $\begin{array}{c}\text { Hold Time, } \\
\mathrm{h}\end{array}$ & 850 & 875 & 900 & 915 & 925 & 950 \\
\hline 1 & NLS-2 $^{\text {a }}$ & NLS-3 & NLS-4 & NLS-1 & NLS-5 & NLS-7 \\
\hline 4 & NLS-15 & NLS-14 & NLS-10 & NLS-13 & NLS-12 & NLS-9R \\
\hline 8 & NLS-17 & NLS-19 & NLS-20 & NLS-21 & NLS-22 & NLS-25 \\
\hline 16 & NLS-26 & NLS-28 & NLS-29 & NLS-8 & NLS-30 & NLS-31 \\
\hline 24 & NLS-24 & NLS-33 & NLS-34 & NLS-36 & NLS-35 & NLS-32 \\
\hline 36 & NLS-27 & NLS-23 & NLS-18 & NLS-16 & NLS-11 & NLS-6 \\
\hline
\end{tabular}

${ }^{a}$ Cell entries give T-t PC CWF product identifiers. 
Table 32. Densities of the 36 T-t PC CWF Products, in $\mathrm{kg} / \mathrm{m}^{3}$

\begin{tabular}{|c|c|c|c|c|c|c|}
\hline \multirow{2}{*}{ Time, h } & \multicolumn{6}{|c|}{ Processing Temperature, ${ }^{\circ} \mathrm{C}$} \\
\cline { 2 - 7 } & 850 & 875 & 900 & 915 & 925 & 950 \\
\hline 1 & 1160 & 1260 & 1340 & 1480 & 1490 & 1670 \\
\hline 4 & 1320 & 1480 & 1620 & 1710 & 1830 & 2010 \\
\hline 8 & 1490 & 1630 & 1780 & 1890 & 1950 & 2020 \\
\hline 16 & 1580 & 1700 & 1810 & 1950 & 1970 & 1970 \\
\hline 24 & 1640 & 1780 & 1930 & 2000 & 2010 & 1930 \\
\hline 36 & 1650 & 1840 & 1970 & 2010 & 1990 & 1910 \\
\hline
\end{tabular}


Table 33. Measured d-Spacings for CWF Materials Processed for 1 Hour

\begin{tabular}{|c|c|c|c|c|c|c|c|c|c|c|c|c|}
\hline \multicolumn{2}{|c|}{$\begin{array}{c}850^{\circ} \mathrm{C} \\
\text { CWF Sample } \\
\text { PC04101 } \\
\text { XRD spt NST1902 }\end{array}$} & \multicolumn{2}{|c|}{$\begin{array}{c}875^{\circ} \mathrm{C} \\
\text { CWF Sample } \\
\text { PC04201 } \\
\text { XRD spt NST1903 }\end{array}$} & \multicolumn{2}{|c|}{$\begin{array}{c}900^{\circ} \mathrm{C} \\
\text { CWF Sample } \\
\text { PC04301 } \\
\text { XRD spt NST1904 }\end{array}$} & \multicolumn{2}{|c|}{$\begin{array}{c}915^{\circ} \mathrm{C} \\
\text { CWF Sample } \\
\text { PC04001 } \\
\text { XRD spt NST1901 }\end{array}$} & \multicolumn{2}{|c|}{$\begin{array}{c}925^{\circ} \mathrm{C} \\
\text { CWF Sample } \\
\text { PC04401 } \\
\text { XRD spt NST1905 }\end{array}$} & \multicolumn{2}{|c|}{$\begin{array}{c}950^{\circ} \mathrm{C} \\
\text { CWF Sample } \\
\text { PC04601 } \\
\text { XRD spt NST1907 }\end{array}$} & \multirow[t]{2}{*}{ ID } \\
\hline $\mathrm{d}(\mathrm{A})$ & $1 \%$ & $d(A)$ & $1 \%$ & $\mathrm{~d}(\mathrm{~A})$ & $1 \%$ & $\mathrm{~d}(\mathrm{~A})$ & $1 \%$ & $d(A)$ & $1 \%$ & $\mathrm{~d}(\mathrm{~A})$ & $1 \%$ & \\
\hline 6.263 & 25.1 & 6.235 & 21.6 & 6.242 & 23.9 & 6.301 & 27.2 & 6.240 & 24.6 & 6.282 & 23.6 & $S$ \\
\hline 4.426 & 7.6 & 4.414 & 6.5 & 4.417 & 6.8 & 4.446 & 7.0 & 4.416 & 7.7 & 4.438 & 7.0 & S \\
\hline 4.187 & 0.9 & 4.181 & 0.7 & 4.177 & 0.8 & & & & & & & $\mathrm{~N}$ \\
\hline 3.954 & 0.9 & & & 3.950 & 0.9 & 3.976 & 0.8 & 3.957 & 0.9 & & & $S$ \\
\hline 3.847 & 2.1 & 3.834 & 1.5 & 3.839 & 1.2 & & & & & & & $\mathrm{~N}$ \\
\hline 3.614 & 100 & 3.603 & 100 & 3.608 & 100 & 3.626 & 100 & 3.608 & 100 & 3.622 & 100 & $\underline{\mathbf{S}}$ \\
\hline 3.445 & 0.5 & & & & & & & 3.333 & 0.7 & & & $-^{a}$ \\
\hline 3.275 & 1.6 & 3.266 & 1.2 & 3.262 & 0.9 & & & 3.261 & 0.7 & & & $\mathrm{H}, \mathrm{N}$ \\
\hline 3.007 & 2.2 & 3.005 & 2.4 & 3.004 & 1.3 & & & 3.002 & 0.8 & & & $\mathbf{N}$ \\
\hline 2.885 & 1.0 & & & 2.884 & 0.5 & & & & & & & $\mathrm{~N}$ \\
\hline 2.798 & 8.5 & 2.794 & 9.3 & 2.795 & 8.1 & 2.807 & 8.0 & 2.795 & 9.1 & 2.803 & 9.2 & $\mathrm{~S}, \mathrm{H}$ \\
\hline 2.554 & 20.2 & 2.551 & 203 & 2.552 & 17.8 & 2.561 & 19.4 & 2.551 & 19.3 & 2.558 & 20.0 & $\mathrm{~S}$ \\
\hline 2.365 & 17.8 & 2.361 & 20.6 & 2.363 & 18.1 & 2.372 & 18.9 & 2.363 & 19.5 & 2.369 & 20.7 & S \\
\hline 2.308 & 0.5 & & & & & & & & & & & $\mathrm{~N}$ \\
\hline 2.212 & 0.9 & 2.210 & 1.1 & 2.211 & 1.1 & 2.218 & 1.2 & 2.212 & 1.3 & 2.217 & 1.2 & S \\
\hline 2.085 & 26.3 & 2.083 & 30.2 & 2.085 & 24.3 & 2.090 & 26.8 & 2.085 & 26.8 & 2.089 & 27.2 & S \\
\hline 1.979 & 4.0 & 1.977 & 4.7 & 1.977 & 4.0 & 1.999 & 1.3 & 1.994 & 1.5 & 1.997 & 2.1 & $\mathrm{H}$ \\
\hline & & & & & & 1.983 & 4.2 & 1.977 & 4.6 & 1.982 & 4.5 & S \\
\hline 1.886 & 3.4 & 1.884 & 3.9 & 1.886 & 3.8 & 1.890 & 3.7 & 1.886 & 3.8 & 1.889 & 3.3 & $S$ \\
\hline 1.806 & 2.7 & 1.804 & 3.6 & 1.806 & 2.7 & 1.810 & 2.7 & 1.806 & 3.1 & 1.809 & 3.1 & S \\
\hline 1.735 & 2.9 & 1.734 & 3.8 & 1.735 & 2.9 & 1.739 & 3.2 & 1.735 & 3.3 & 1.738 & 3.3 & $\mathrm{~S}$ \\
\hline 1.616 & 2.5 & 1.614 & 3.0 & 1.614 & 2.3 & 1.618 & 2.4 & 1.615 & 2.4 & 1.617 & 2.6 & S \\
\hline 1.534 & 8.9 & 1.563 & 10.8 & 1.564 & 9.1 & 1.567 & 8.8 & 1.564 & 9.7 & 1.567 & 9.5 & $S$ \\
\hline 1.517 & 4.0 & 1.517 & 5.4 & 1.518 & 4.0 & 1.520 & 4.3 & 1.518 & 4.6 & 1.520 & 4.6 & $\mathrm{~S}$ \\
\hline 1.474 & 6.0 & 1.473 & $\begin{array}{l}7.4 \\
7.6\end{array}$ & 1.474 & 6.0 & 1.477 & 6.4 & 1.474 & 7.0 & 1.477 & 7.0 & $S$ \\
\hline
\end{tabular}

${ }^{\mathrm{a}}$ Unidentified peak. 
Table 34. Measured d-Spacings for CWF Materials Processed for 4 Hours

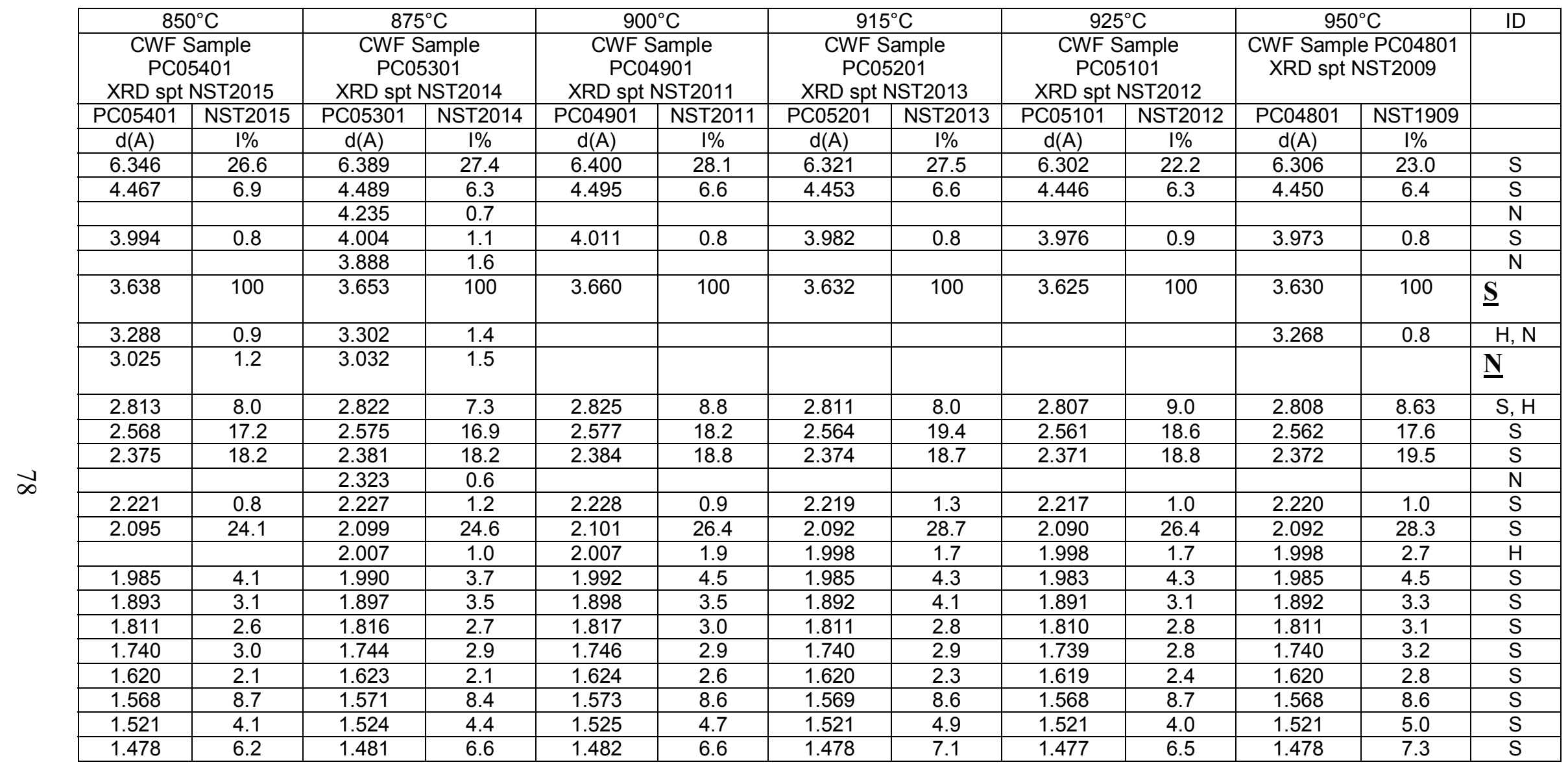


Table 35. Measured d-Spacings for CWF Materials Processed for 8 Hours

\begin{tabular}{|c|c|c|c|c|c|c|c|c|c|c|c|c|}
\hline \multirow{2}{*}{\multicolumn{2}{|c|}{$\begin{array}{c}850^{\circ} \mathrm{C} \\
\text { CWF Sample PC07501 } \\
\text { XRD spt AHE0227I }\end{array}$}} & \multirow{2}{*}{\multicolumn{2}{|c|}{$\begin{array}{c}875^{\circ} \mathrm{C} \\
\text { CWF Sample } \\
\text { PC06001 } \\
\text { XRD spt AHE02273 }\end{array}$}} & \multirow{2}{*}{\multicolumn{2}{|c|}{$\begin{array}{c}900^{\circ} \mathrm{C} \\
\text { CWF Sample } \\
\text { PC06101 } \\
\text { XRD spt AHE02274 }\end{array}$}} & \multirow{2}{*}{\multicolumn{2}{|c|}{$\begin{array}{c}915^{\circ} \mathrm{C} \\
\text { CWF Sample } \\
\text { PC06201 } \\
\text { XRD spt AHE02275 }\end{array}$}} & \multirow{2}{*}{\multicolumn{2}{|c|}{$\begin{array}{c}925^{\circ} \mathrm{C} \\
\text { CWF Sample } \\
\text { PC06301 } \\
\text { XRD spt AHE02276 }\end{array}$}} & \multirow{2}{*}{\multicolumn{2}{|c|}{$\begin{array}{c}950^{\circ} \mathrm{C} \\
\text { CWF Sample } \\
\text { PC06601 } \\
\text { XRD spt AHE02279 }\end{array}$}} & \multirow[t]{3}{*}{ ID } \\
\hline & & & & & & & & & & & & \\
\hline$d(A)$ & $1 \%$ & $\mathrm{~d}(\mathrm{~A})$ & $1 \%$ & $\mathrm{~d}(\mathrm{~A})$ & $1 \%$ & $d(A)$ & $1 \%$ & $d(A)$ & $1 \%$ & $d(A)$ & $1 \%$ & \\
\hline 6.344 & 26.0 & 6.371 & 27.7 & 6.375 & 21.5 & 6.240 & 19.3 & 6.366 & 19.7 & 6.264 & 18.3 & $S$ \\
\hline \multirow[t]{2}{*}{4.469} & 6.2 & 4.481 & 6.7 & 4.482 & 6.0 & 4.417 & 5.8 & 4.478 & 6.6 & 4.429 & 6.3 & $S$ \\
\hline & & 4.002 & 1.1 & 4.003 & 0.9 & & & 3.996 & 1.3 & 3.960 & 1.2 & $S$ \\
\hline \multirow[t]{3}{*}{3.639} & 100 & 3.647 & 100 & 3.652 & 100.0 & 3.608 & 100 & 3.647 & 100 & 3.617 & 100 & $\underline{\mathbf{S}}$ \\
\hline & & 3.290 & 0.8 & & & & & & & 3.263 & 1.6 & $\mathrm{H}, \mathrm{N}$ \\
\hline & & 3.027 & 0.7 & & & & & & & 3.002 & 1.1 & $\mathbf{N}$ \\
\hline \multirow[t]{2}{*}{2.816} & 8.8 & 2.820 & 8.8 & 2.821 & 8.9 & 2.815 & 5.2 & 2.820 & 8.6 & 2.803 & 9.0 & $\mathbf{H}$ \\
\hline & & & & & & 2.798 & 8.3 & & & & & $S$ \\
\hline 2.568 & 19.1 & 2.572 & 20.0 & 2.573 & 20.0 & 2.553 & 18.8 & 2.572 & 18.9 & 2.557 & 21.7 & $\mathrm{~S}$ \\
\hline 2.376 & 20.4 & 2.380 & 18.3 & 2.381 & 19.3 & 2.363 & 17.8 & 2.380 & 19.0 & 2.368 & 20.4 & $S$ \\
\hline 2.224 & 1.2 & 2.225 & 1.3 & 2.226 & 1.0 & 2.212 & 1.3 & 2.225 & 1.1 & 2.217 & 1.0 & $S$ \\
\hline 2.095 & 30.4 & 2.097 & 28.8 & 2.099 & 27.3 & 2.085 & 27.5 & 2.097 & 26.3 & 2.088 & 30.2 & S \\
\hline 2.002 & 2.0 & 2.006 & 1.6 & 2.004 & 2.3 & 1.992 & 2.1 & 2.004 & 2.3 & 1.994 & 3.5 & $\mathrm{H}$ \\
\hline 1.986 & 5.1 & 1.989 & 3.9 & 1.990 & 4.2 & 1.979 & 3.4 & 1.990 & 4.2 & 1.982 & 4.7 & $S$ \\
\hline 1.894 & 3.9 & 1.896 & 3.6 & 1.897 & 3.4 & 1.886 & 3.7 & 1.896 & 3.5 & 1.890 & 3.6 & $S$ \\
\hline 1.813 & 3.2 & 1.814 & 2.8 & 1.816 & 2.9 & 1.806 & 2.9 & 1.815 & 3.1 & 1.809 & 2.7 & S \\
\hline 1.742 & 3.3 & 1.743 & 3.2 & 1.745 & 3.3 & 1.736 & 2.8 & 1.744 & 3.1 & 1.738 & 3.7 & $S$ \\
\hline \multirow[t]{2}{*}{1.622} & 2.8 & 1.623 & 2.5 & 1.623 & 2.2 & 1.616 & 2.1 & 1.623 & 2.2 & 1.628 & 0.9 & $\mathrm{H}$ \\
\hline & & & & & & & & & & 1.618 & 3.0 & $\mathrm{~N}$ \\
\hline 1.570 & 10.4 & 1.570 & 9.7 & 1.571 & 9.3 & 1.565 & 8.4 & 1.571 & 9.1 & 1.567 & 10.1 & $S$ \\
\hline 1.523 & 4.7 & 1.523 & 4.6 & 1.524 & 3.9 & 1.519 & 4.3 & 1.524 & 4.3 & 1.520 & 5.1 & S \\
\hline 1.480 & 7.9 & 1.480 & 7.3 & 1.481 & 7.3 & 1.475 & 6.6 & 1.481 & 6.8 & 1.477 & 8.1 & $S$ \\
\hline
\end{tabular}


Table 36. Measured d-Spacings for CWF Materials Processed for 16 Hours

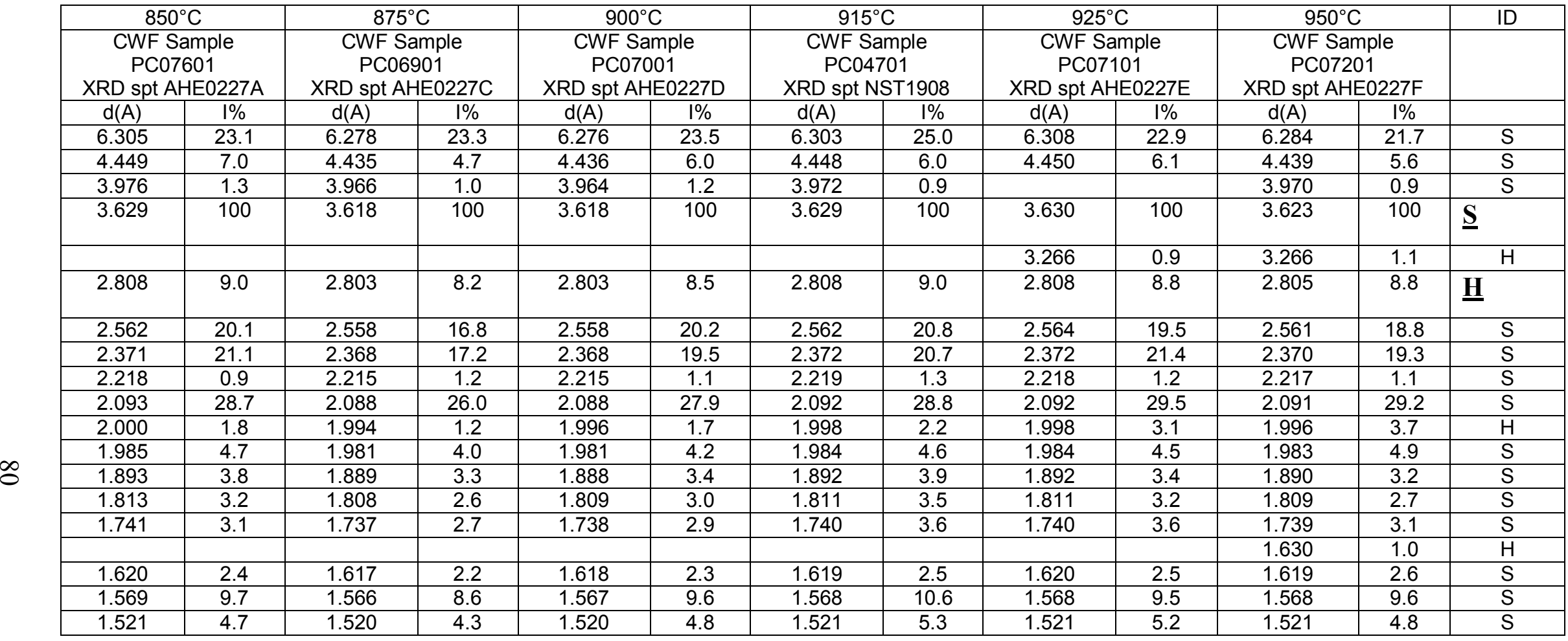


Table 37. Measured d-Spacings for CWF Materials Processed for 24 Hours

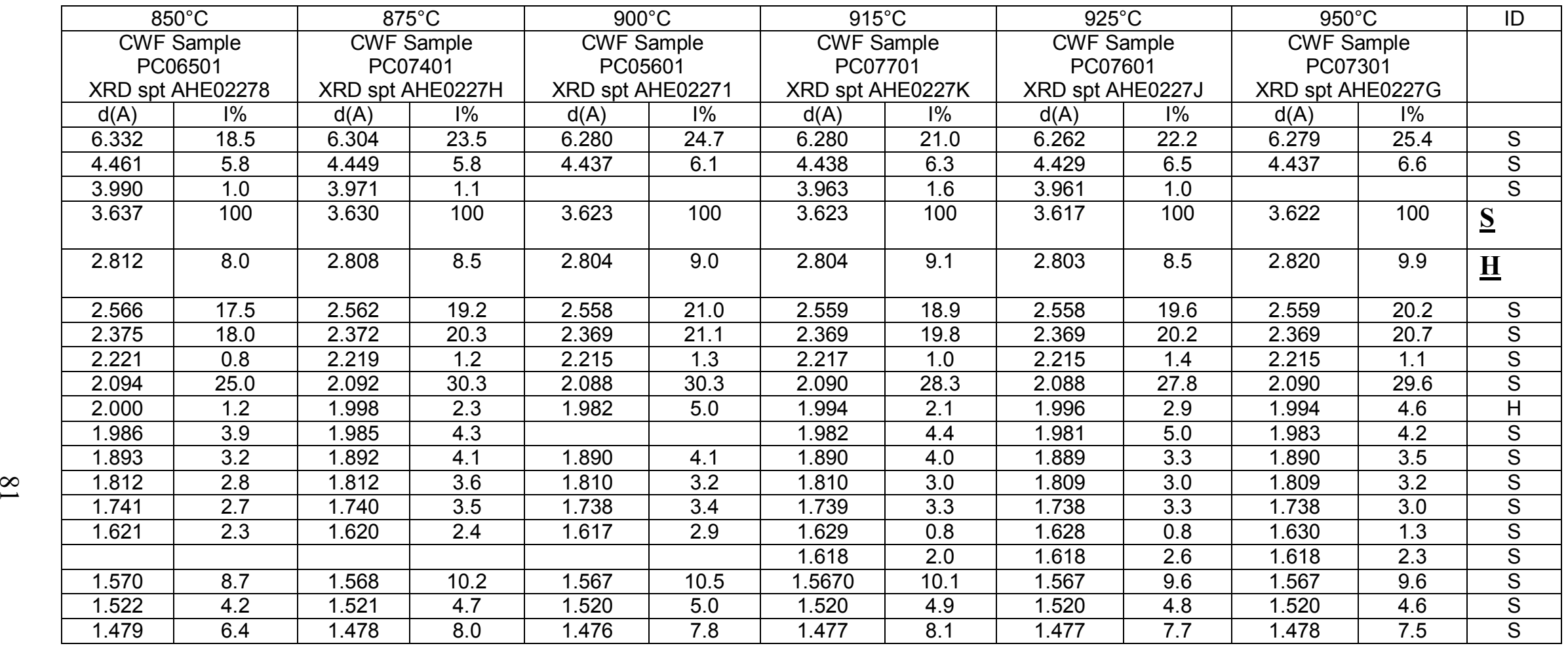


Table 38. Measured d-Spacings for CWF Materials Processed for 36 Hours

\begin{tabular}{|c|c|c|c|c|c|c|c|c|c|c|c|c|}
\hline \multirow{2}{*}{\multicolumn{2}{|c|}{$\begin{array}{c}850^{\circ} \mathrm{C} \\
\text { CWF Sample } \\
\text { PC06801 } \\
\text { XRD spt AHE0227B }\end{array}$}} & \multirow{2}{*}{\multicolumn{2}{|c|}{$\begin{array}{c}875^{\circ} \mathrm{C} \\
\text { CWF Sample } \\
\text { PC06401 } \\
\text { XRD spt AHE02277 }\end{array}$}} & \multirow{2}{*}{\multicolumn{2}{|c|}{$\begin{array}{c}900^{\circ} \mathrm{C} \\
\text { CWF Sample } \\
\text { PC05901 } \\
\text { XRD spt AHE02272 }\end{array}$}} & \multirow{2}{*}{\multicolumn{2}{|c|}{$\begin{array}{c}915^{\circ} \mathrm{C} \\
\text { CWF Sample } \\
\text { PC05501 } \\
\text { XRD spt AHE02542 }\end{array}$}} & \multirow{2}{*}{\multicolumn{2}{|c|}{$\begin{array}{c}925^{\circ} \mathrm{C} \\
\text { CWF Sample } \\
\text { PC05001 } \\
\text { XRD spt AHE02541 }\end{array}$}} & \multirow{2}{*}{\multicolumn{2}{|c|}{$\begin{array}{c}950^{\circ} \mathrm{C} \\
\text { CWF Sample } \\
\text { PC04501 } \\
\text { XRD spt NST1906 }\end{array}$}} & \multirow[t]{3}{*}{ ID } \\
\hline & & & & & & & & & & & & \\
\hline $\mathrm{d}(\mathrm{A})$ & $1 \%$ & $\mathrm{~d}(\mathrm{~A})$ & $1 \%$ & $\mathrm{~d}(\mathrm{~A})$ & $1 \%$ & $\mathrm{~d}(\mathrm{~A})$ & $1 \%$ & $\mathrm{~d}(\mathrm{~A})$ & $1 \%$ & $d(A)$ & $1 \%$ & \\
\hline 6.304 & 27.7 & 6.356 & 19.3 & 6.324 & 24.7 & 6.169 & 19.2 & 6.278 & 20.4 & 6.303 & 21.3 & $\mathrm{~S}$ \\
\hline 4.448 & 6.8 & 4.474 & 5.8 & 4.457 & 6.0 & 4.384 & 5.6 & 4.435 & 6.4 & 4.449 & 6.6 & $\mathrm{~S}$ \\
\hline 3.975 & 1.0 & 3.998 & 1.1 & & & 3.930 & 1.3 & 3.967 & 0.9 & & & S \\
\hline 3.629 & 100 & 3.646 & 100 & 3.633 & 100 & 3.588 & 100 & 3.621 & 100 & 3.630 & 100 & $\underline{\mathbf{S}}$ \\
\hline & & & & & & 3.241 & 1.2 & 3.259 & 1.1 & & & $\mathrm{H}$ \\
\hline 2.807 & 8.7 & 2.819 & 1.9 & 2.811 & 8.4 & 2.787 & 10.1 & 2.804 & 9.6 & 2.809 & 9.1 & H \\
\hline 2.562 & 18.7 & 2.572 & 17.9 & 2.562 & 19.9 & 2.544 & 22.7 & 2.558 & 21.7 & 2.562 & 20.0 & $\mathrm{~S}$ \\
\hline 2.372 & 19.7 & 2.380 & 17.7 & 2.374 & 20.0 & 2.357 & 24.4 & 2.369 & 21.9 & 2.372 & 21.2 & S \\
\hline 2.219 & 1.0 & 2.224 & 1.1 & 2.220 & 1.2 & 2.206 & 1.3 & 2.216 & 1.2 & 2.218 & 1.2 & $S$ \\
\hline 2.092 & 25.6 & 2.097 & 25.6 & 2.092 & 28.7 & 2.080 & 35.6 & 2.090 & 30.8 & 2.092 & 29.8 & S \\
\hline 1.998 & 1.3 & 2.004 & 1.4 & 1.998 & 2.4 & 1.983 & 2.8 & 1.996 & 3.1 & 1.994 & 3.2 & $\mathrm{H}$ \\
\hline 1.983 & 4.2 & 1.989 & 3.8 & 1.985 & 4.6 & 1.974 & 5.2 & 1.982 & 4.5 & 1.985 & 5.0 & $\mathrm{~S}$ \\
\hline 1.892 & 3.3 & 1.896 & 3.3 & 1.892 & 3.4 & 1.883 & 4.6 & 1.890 & 4.3 & 1.892 & 4.0 & $\mathrm{~S}$ \\
\hline 1.811 & 2.9 & 1.815 & 2.6 & 1.812 & 3.4 & 1.802 & 4.4 & 1.809 & 2.9 & 1.812 & 3.2 & $\mathrm{~S}$ \\
\hline 1.740 & 3.1 & 1.743 & 2.4 & 1.740 & 3.1 & 1.733 & 3.7 & 1.739 & 3.2 & 1.740 & 3.6 & S \\
\hline 1.618 & 2.1 & 1.622 & 2.1 & 1.631 & 0.5 & & & 1.630 & 0.7 & 1.631 & 0.9 & $\mathrm{~S}$ \\
\hline & & & & 1.621 & 2.6 & 1.613 & 3.3 & 1.618 & 2.6 & 1.620 & 3.0 & S \\
\hline 1.568 & 9.0 & 1.570 & 8.0 & 1.569 & 9.8 & 1.563 & 11.6 & 1.567 & 10.0 & 1.568 & 10.1 & $\mathrm{~S}$ \\
\hline 1.521 & 4.4 & 1.524 & 3.9 & 1.522 & 4.5 & 1.516 & 7.1 & 1.520 & 4.7 & 1.522 & 5.0 & S \\
\hline 1.478 & 6.6 & 1.481 & 6.6 & 1.479 & 7.6 & 1.473 & 10.3 & 1.477 & 8.1 & 1.479 & 8.1 & $\mathrm{~S}$ \\
\hline
\end{tabular}


Table 39. Test Data for Triplicate PCT with T-t PC CWF Materials

\begin{tabular}{|c|c|c|c|c|c|c|c|c|}
\hline \multirow{2}{*}{$\begin{array}{c}\text { Hold } \\
\text { Time, h }\end{array}$} & \multirow{2}{*}{$\begin{array}{l}\text { PC CWF } \\
\text { Identifier }\end{array}$} & \multicolumn{3}{|c|}{ RWS Step } & \multicolumn{4}{|c|}{ PCT Step } \\
\hline & & Mass, $\mathrm{g}$ & Vol., $\mathrm{mL}$ & $\mathrm{S} / \mathrm{V}, \mathrm{m}^{-1}$ & Mass, $\mathrm{g}$ & Vol., mL & $\mathrm{S} / \mathrm{V}, \mathrm{m}^{-1}$ & $\mathrm{pH}$ \\
\hline \multicolumn{9}{|c|}{ Processing Temperature $=850^{\circ} \mathrm{C}$} \\
\hline 1 & NLS-2a & 2.25 & 23.28 & 2223 & 1.00 & 10.00 & 2300 & 8.95 \\
\hline 1 & NLS-2b & 2.28 & 22.79 & 2301 & 1.00 & 9.94 & 2314 & 8.91 \\
\hline 1 & NLS-2c & 2.30 & 23.04 & 2296 & 1.00 & 9.95 & 2312 & 8.93 \\
\hline 4 & NLS-15a & 3.00 & 30.04 & 2297 & 1.57 & 15.68 & 2303 & 9.09 \\
\hline 4 & NLS-15b & 3.00 & 30.01 & 2299 & 1.50 & 15.00 & 2300 & 9.06 \\
\hline 4 & NLS-15c & 3.00 & 30.06 & 2295 & 1.49 & 14.89 & 2302 & 9.07 \\
\hline 8 & NLS-17a & 3.01 & 37.44 & 1849 & 1.53 & 15.30 & 2300 & 9.01 \\
\hline 8 & NLS-17b & 3.00 & 30.03 & 2298 & 1.50 & 14.98 & 2303 & 7.99 \\
\hline 8 & NLS-17c & 3.00 & 30.11 & 2292 & 1.54 & 15.47 & 2290 & 8.93 \\
\hline 16 & NLS-26a & 3.05 & 31.21 & 2248 & 1.96 & 19.58 & 2302 & 9.21 \\
\hline 16 & NLS-26b & 3.06 & 30.63 & 2298 & 1.96 & 19.59 & 2301 & 9.07 \\
\hline 16 & NLS-26c & 3.02 & 31.53 & 2203 & 1.98 & 19.78 & 2302 & 9.01 \\
\hline 24 & NLS-24a & 2.41 & 24.13 & 2297 & 1.55 & 15.46 & 2306 & 9.07 \\
\hline 24 & NLS-24b & 2.45 & 25.00 & 2254 & 1.52 & 15.19 & 2302 & 9.06 \\
\hline 24 & NLS-24c & 2.46 & 24.90 & 2272 & 1.51 & 15.13 & 2295 & 9.04 \\
\hline 36 & NLS-27a & 3.02 & 31.30 & 2219 & 1.90 & 18.98 & 2302 & 8.96 \\
\hline 36 & NLS-27b & 3.06 & 31.93 & 2204 & 1.93 & 19.50 & 2276 & 9.01 \\
\hline 36 & NLS-27c & 3.13 & 33.31 & 2161 & 1.96 & 19.58 & 2302 & 8.99 \\
\hline \multicolumn{9}{|c|}{ Processing Temperature $=875^{\circ} \mathrm{C}$} \\
\hline 1 & NLS-3a & 2.53 & 25.30 & 2300 & 1.00 & 9.98 & 2305 & 9.04 \\
\hline 1 & NLS-3b & 2.47 & 24.73 & 2297 & 1.00 & 9.94 & 2314 & 8.99 \\
\hline 1 & NLS-3c & 2.52 & 25.25 & 2295 & 1.00 & 9.86 & 2333 & 9.00 \\
\hline 4 & NLS-14a & 3.00 & 30.04 & 2297 & 1.51 & 15.30 & 2270 & 9.01 \\
\hline 4 & NLS-14b & 3.00 & 30.19 & 2286 & 1.57 & 15.68 & 2303 & 9.05 \\
\hline 4 & NLS-14c & 2.86 & 26.60 & 2473 & 1.49 & 14.88 & 2303 & 9.03 \\
\hline 8 & NLS-19a & 3.04 & 30.40 & 2300 & 1.55 & 15.47 & 2304 & 9.02 \\
\hline 8 & NLS-19b & 3.03 & 30.27 & 2302 & 1.51 & 15.27 & 2274 & 9.00 \\
\hline 8 & NLS-19c & 3.11 & 31.11 & 2299 & 1.51 & 15.09 & 2302 & 9.02 \\
\hline 16 & NLS-28a & 2.41 & 24.13 & 2297 & 2.15 & 21.51 & 2299 & 8.88 \\
\hline 16 & NLS-28b & 2.45 & 25.00 & 2254 & 1.93 & 19.34 & 2295 & 8.87 \\
\hline 16 & NLS-28c & 2.46 & 24.90 & 2272 & 2.07 & 20.70 & 2300 & 8.87 \\
\hline 24 & NLS-33a & 3.02 & 30.23 & 2298 & 1.92 & 19.24 & 2295 & 8.89 \\
\hline 24 & NLS-33b & 3.12 & 31.26 & 2296 & 2.01 & 20.43 & 2263 & 8.87 \\
\hline 24 & NLS-33c & 2.99 & 29.66 & 2319 & 2.01 & 20.08 & 2302 & 8.88 \\
\hline 36 & NLS-23a & 3.02 & 31.30 & 2219 & 1.94 & 19.37 & 2304 & 9.15 \\
\hline 36 & NLS-23b & 3.06 & 31.93 & 2204 & 1.95 & 19.77 & 2269 & 9.19 \\
\hline 36 & NLS-23c & 3.13 & 33.31 & 2161 & 1.95 & 19.48 & 2302 & 9.08 \\
\hline \multicolumn{9}{|c|}{ Processing Temperature $=900^{\circ} \mathrm{C}$} \\
\hline 1 & NLS-4a & 2.52 & 25.21 & 2299 & 1.50 & 14.98 & 2303 & 8.84 \\
\hline 1 & NLS-4b & 2.64 & 26.52 & 2290 & 1.50 & 14.99 & 2302 & 8.81 \\
\hline 1 & NLS-4c & 2.55 & 25.52 & 2298 & 1.50 & 14.98 & 2303 & 8.98 \\
\hline 4 & NLS-10a & 2.73 & 27.35 & 2296 & 1.50 & 15.11 & 2283 & 9.06 \\
\hline 4 & NLS-10b & 2.83 & 28.70 & 2268 & 1.49 & 14.90 & 2300 & 9.03 \\
\hline 4 & NLS-10c & 2.83 & 28.40 & 2292 & 1.49 & 14.91 & 2298 & 9.00 \\
\hline 8 & NLS-20a & 2.56 & 25.60 & 2300 & 1.53 & 15.30 & 2300 & 9.03 \\
\hline 8 & NLS-20b & 2.52 & 25.17 & 2303 & 1.56 & 15.83 & 2267 & 8.98 \\
\hline 8 & NLS-20c & 2.59 & 25.95 & 2296 & 1.53 & 15.35 & 2293 & 9.01 \\
\hline 16 & NLS-29a & 2.86 & 30.68 & 2144 & 2.03 & 20.29 & 2301 & 9.02 \\
\hline 16 & NLS-29b & 3.04 & 33.15 & 2109 & 2.11 & 21.09 & 2301 & 9.03 \\
\hline 16 & NLS-29c & 2.84 & 28.88 & 2262 & 2.00 & 20.00 & 2300 & 8.99 \\
\hline 24 & NLS-34a & 2.45 & 24.54 & 2296 & 2.15 & 21.51 & 2299 & 9.02 \\
\hline 24 & NLS-34b & 2.46 & 25.08 & 2256 & 1.94 & 19.43 & 2296 & 8.99 \\
\hline 24 & NLS-34c & 2.52 & 26.78 & 2164 & 2.12 & 21.20 & 2300 & 8.98 \\
\hline 36 & NLS-18a & 3.00 & 30.02 & 2298 & 1.51 & 15.17 & 2289 & 9.05 \\
\hline 36 & NLS-18b & 3.00 & 30.00 & 2300 & 1.51 & 15.19 & 2286 & 9.05 \\
\hline 36 & NLS-18c & 3.01 & 30.11 & 2299 & 1.50 & 15.03 & 2295 & 9.09 \\
\hline
\end{tabular}


Table 39. (Contd.)

\begin{tabular}{|c|c|c|c|c|c|c|c|c|}
\hline \multirow{2}{*}{$\begin{array}{c}\text { Hold } \\
\text { Time, } \mathrm{h}\end{array}$} & \multirow{2}{*}{$\begin{array}{l}\text { PC CWF } \\
\text { Identifier }\end{array}$} & \multicolumn{3}{|c|}{ RWS Step } & \multicolumn{4}{|c|}{ PCT Step } \\
\hline & & Mass, $\mathrm{g}$ & Vol., $\mathrm{mL}$ & $\mathrm{S} / \mathrm{V}, \mathrm{m}^{-1}$ & Mass, $\mathrm{g}$ & Vol., $\mathrm{mL}$ & $\mathrm{S} / \mathrm{V}, \mathrm{m}^{-1}$ & $\mathrm{pH}$ \\
\hline \multicolumn{9}{|c|}{ Processing Temperature $=915^{\circ} \mathrm{C}$} \\
\hline 1 & NLS-1a & 2.14 & 21.44 & 2296 & 1.50 & 14.98 & 2303 & 8.52 \\
\hline 1 & NLS-1b & 2.22 & 22.31 & 2289 & 1.50 & 14.97 & 2305 & 8.94 \\
\hline 1 & NLS-1c & 2.20 & 21.98 & 2302 & 1.46 & 14.57 & 2305 & 8.74 \\
\hline 4 & NLS-13a & 3.00 & 30.10 & 2292 & 1.50 & 14.97 & 2305 & 8.97 \\
\hline 4 & NLS-13b & 2.99 & 29.93 & 2298 & 1.49 & 14.86 & 2306 & 9.04 \\
\hline 4 & NLS-13c & 2.99 & 30.04 & 2289 & 1.50 & 14.96 & 2306 & 9.01 \\
\hline 8 & NLS-21a & 1.54 & 15.43 & 2296 & 1.54 & 15.42 & 2297 & 9.02 \\
\hline 8 & NLS-21b & 1.56 & 16.40 & 2188 & 1.56 & 16.39 & 2189 & 9.02 \\
\hline 8 & NLS-21c & 1.53 & 15.36 & 2291 & 1.53 & 15.36 & 2291 & 8.94 \\
\hline 16 & NLS-8a & 2.28 & 22.90 & 2290 & 1.00 & 10.00 & 2300 & 8.91 \\
\hline 16 & NLS-8b & 2.25 & 22.46 & 2304 & 1.00 & 10.00 & 2300 & 9.04 \\
\hline 16 & NLS-8c & 2.27 & 22.73 & 2297 & 1.00 & 10.00 & 2300 & 8.98 \\
\hline 24 & NLS-36a & 2.50 & 24.98 & 2302 & 2.11 & 21.10 & 2300 & 8.97 \\
\hline 24 & NLS-36b & 2.54 & 25.47 & 2294 & 2.06 & 20.59 & 2301 & 8.94 \\
\hline 24 & NLS-36c & 2.51 & 25.08 & 2302 & 2.00 & 19.97 & 2303 & 8.91 \\
\hline 36 & NLS-16a & 3.00 & 30.01 & 2299 & 1.59 & 15.87 & 2304 & 9.17 \\
\hline 36 & NLS-16b & 3.00 & 31.20 & 2212 & 1.49 & 14.88 & 2303 & 9.10 \\
\hline 36 & NLS-16c & 3.00 & 29.99 & 2301 & 1.52 & 15.19 & 2302 & 9.17 \\
\hline \multicolumn{9}{|c|}{ Processing Temperature $=925^{\circ} \mathrm{C}$} \\
\hline 1 & NLS-5a & 2.08 & 20.74 & 2307 & 1.00 & 10.00 & 2300 & 9.09 \\
\hline 1 & NLS-5b & 2.09 & 20.83 & 2308 & 1.00 & 9.98 & 2305 & 9.01 \\
\hline 1 & NLS-5c & 2.11 & 21.98 & 2208 & 1.00 & 9.98 & 2305 & 8.96 \\
\hline 4 & NLS-12a & 2.46 & 24.63 & 2297 & 1.52 & 15.32 & 2282 & 8.96 \\
\hline 4 & $\mathrm{NLS}-12 \mathrm{~b}$ & 2.45 & 24.50 & 2300 & 1.45 & 14.50 & 2300 & 8.95 \\
\hline 4 & NLS-12c & 2.30 & 23.00 & 2300 & 1.55 & 15.50 & 2300 & 8.94 \\
\hline 8 & NLS-22a & 3.05 & 31.21 & 2248 & 1.93 & 19.44 & 2283 & 8.90 \\
\hline 8 & NLS-22b & 3.06 & 30.63 & 2298 & 1.91 & 19.09 & 2301 & 8.95 \\
\hline 8 & NLS-22c & 3.02 & 31.53 & 2203 & 1.96 & 19.62 & 2298 & 8.95 \\
\hline 16 & NLS-30a & 2.82 & 28.49 & 2277 & 1.94 & 19.38 & 2302 & 8.96 \\
\hline 16 & NLS-30b & 2.66 & 26.66 & 2295 & 1.96 & 19.61 & 2299 & 9.03 \\
\hline 16 & NLS-30c & 2.62 & 26.15 & 2304 & 1.97 & 19.76 & 2293 & 9.03 \\
\hline 24 & NLS-35a & 2.56 & 26.92 & 2187 & 1.92 & 19.38 & 2279 & 8.99 \\
\hline 24 & NLS-35b & 2.60 & 26.03 & 2297 & 1.94 & 19.52 & 2286 & 8.96 \\
\hline 24 & NLS-35c & 2.62 & 26.21 & 2299 & 1.96 & 19.59 & 2301 & 8.96 \\
\hline 36 & NLS-11a & 2.64 & 26.53 & 2289 & 1.50 & 15.04 & 2294 & 9.03 \\
\hline 36 & NLS-11b & 2.65 & 26.54 & 2297 & 1.49 & 15.25 & 2247 & 8.99 \\
\hline 36 & NLS-11c & 2.61 & 26.13 & 2297 & 1.49 & 14.90 & 2300 & 9.06 \\
\hline \multicolumn{9}{|c|}{ Processing Temperature $=950^{\circ} \mathrm{C}$} \\
\hline 1 & NLS-7a & 2.40 & 23.98 & 2302 & 1.50 & 15.00 & 2300 & 8.19 \\
\hline 1 & NLS-7b & 2.43 & 24.32 & 2298 & 1.50 & 15.06 & 2291 & 8.75 \\
\hline 1 & NLS-7c & 2.48 & 24.93 & 2288 & 1.50 & 15.00 & 2300 & 8.81 \\
\hline 4 & NLS-9Ra & 3.01 & 30.56 & 2265 & 1.66 & 16.73 & 2282 & 9.14 \\
\hline 4 & NLS-9Rb & 3.00 & 30.58 & 2256 & 1.53 & 15.28 & 2303 & 9.06 \\
\hline 4 & NLS-9Rc & 3.01 & 30.09 & 2301 & 1.54 & 15.42 & 2297 & 9.08 \\
\hline 8 & NLS-25a & 1.54 & 15.43 & 2296 & 1.91 & 19.10 & 2300 & 8.91 \\
\hline 8 & NLS-25b & 1.56 & 16.40 & 2188 & 1.91 & 19.09 & 2301 & 8.99 \\
\hline 8 & NLS-25c & 1.53 & 15.36 & 2291 & 1.90 & 18.98 & 2302 & 8.98 \\
\hline 16 & NLS-31a & 2.68 & 27.19 & 2267 & 1.97 & 19.71 & 2299 & 9.09 \\
\hline 16 & NLS-31b & 2.72 & 27.52 & 2273 & 1.98 & 19.79 & 2301 & 8.99 \\
\hline 16 & NLS-31c & 2.71 & 27.76 & 2245 & 1.95 & 19.49 & 2301 & 8.95 \\
\hline 24 & NLS-32a & 2.79 & 27.90 & 2300 & 1.94 & 19.65 & 2271 & 9.02 \\
\hline 24 & NLS-32b & 2.84 & 28.02 & 2331 & 1.96 & 19.60 & 2300 & 8.95 \\
\hline 24 & NLS-32c & 2.65 & 27.05 & 2253 & 1.93 & 19.28 & 2302 & 8.96 \\
\hline 36 & NLS-6a & 2.13 & 21.36 & 2294 & 1.50 & 14.98 & 2303 & 8.85 \\
\hline 36 & NLS-6b & 2.16 & 21.63 & 2297 & 1.50 & 15.04 & 2294 & 8.67 \\
\hline 36 & NLS-6c & 2.25 & 22.50 & 2300 & 1.50 & 14.99 & 2302 & 8.65 \\
\hline
\end{tabular}


Table 40. Results of Triplicate PCT with T-t PC CWF Materials Processed at $850^{\circ} \mathrm{C}$

\begin{tabular}{|c|c|c|c|c|c|c|c|c|c|c|c|c|c|c|c|c|c|}
\hline & $\begin{array}{c}\text { Time, } \\
\mathrm{h}\end{array}$ & $\begin{array}{c}\mathrm{Al}, \\
\mathrm{mg} / \mathrm{L}\end{array}$ & $\begin{array}{c}N L(\mathrm{Al}) \\
\mathrm{g} / \mathrm{m}^{2}\end{array}$ & $\begin{array}{c}\mathrm{B}, \\
\mathrm{mg} / \mathrm{L}\end{array}$ & $\begin{array}{l}N L(B) \\
g / m^{2}\end{array}$ & $\begin{array}{c}\mathrm{Cl}, \\
\mathrm{mg} / \mathrm{L}\end{array}$ & $\begin{array}{c}N L(\mathrm{Cl}) \\
\mathrm{g} / \mathrm{m}^{2}\end{array}$ & $\begin{array}{l}\mathrm{Cs}, \\
\mathrm{mg} / \mathrm{L}\end{array}$ & $\begin{array}{c}N L(\mathrm{Cs}) \\
\mathrm{g} / \mathrm{m}^{2}\end{array}$ & $\begin{array}{c}\text { I, } \\
\mathrm{mg} / \mathrm{L}\end{array}$ & $\begin{array}{l}N L(\mathrm{I}) \\
\mathrm{g} / \mathrm{m}^{2}\end{array}$ & $\begin{array}{c}\mathrm{Li}, \\
\mathrm{mg} / \mathrm{L}\end{array}$ & $\begin{array}{l}N L(\mathrm{Li}) \\
\mathrm{g} / \mathrm{m}^{2}\end{array}$ & $\begin{array}{l}\mathrm{Na}, \\
\mathrm{mg} / \mathrm{L}\end{array}$ & $\begin{array}{c}N L(\mathrm{Na}) \\
\mathrm{g} / \mathrm{m}^{2}\end{array}$ & $\begin{array}{c}\mathrm{Si} \\
\mathrm{mg} / \mathrm{L}\end{array}$ & $\begin{array}{c}N L(\mathrm{Si}) \\
\mathrm{g} / \mathrm{m}^{2}\end{array}$ \\
\hline RWS-NLS-2-a & 1 & 1.96 & 0.00649 & 2.59 & 0.0776 & 7.12 & 0.067 & 0.191 & 0.0589 & 0.0141 & 0.0661 & 0.151 & 0.0154 & 6.38 & 0.0250 & 8.30 & 0.0188 \\
\hline RWS-NLS-2-b & 1 & 1.21 & 0.00388 & & & 6.23 & 0.057 & 0.138 & 0.0409 & 0.0123 & 0.0557 & 0.123 & 0.0122 & 5.06 & 0.0190 & 9.37 & 0.0205 \\
\hline RWS-NLS-2-c & 1 & 2.31 & 0.00738 & & & 6.92 & 0.063 & 0.174 & 0.0519 & 0.0147 & 0.0667 & 0.163 & 0.0161 & 7.24 & 0.0270 & 11.4 & 0.0250 \\
\hline PCT-NLS-2-a & 1 & 34.8 & 0.111 & 46.9 & 1.36 & 28.0 & 0.255 & 0.249 & 0.0741 & 0.0437 & 0.198 & 8.86 & 0.875 & 58.6 & 0.220 & 40.5 & 0.0884 \\
\hline PCT-NLS-2-b & 1 & 33.3 & 0.106 & 48.6 & 1.40 & 26.9 & 0.243 & 0.259 & 0.0767 & 0.0428 & 0.193 & 8.84 & 0.868 & 57.9 & 0.216 & 41.9 & 0.0910 \\
\hline PCT-NLS-2-C & 1 & 33.9 & 0.108 & 45.1 & 1.30 & 25.5 & 0.231 & 0.242 & 0.0716 & 0.0398 & 0.180 & 8.88 & 0.873 & 57.0 & 0.213 & 38.5 & 0.0838 \\
\hline RWS-NLS-15-a & 4 & 0.781 & 0.00250 & 1.08 & 0.0313 & 53.6 & 0.489 & 0.036 & 0.0107 & 0.106 & 0.481 & & & 60.1 & 0.226 & 9.05 & 0.0198 \\
\hline RWS-NLS-15-b & 4 & 0.831 & 0.00266 & & & 45.9 & 0.419 & 0.0315 & 0.0094 & 0.0998 & 0.452 & & & 58.8 & 0.220 & 9.19 & 0.0201 \\
\hline RWS-NLS-15-C & 4 & 0.799 & 0.00256 & & & 47.4 & 0.433 & 0.0336 & 0.0100 & 0.103 & 0.467 & & & 54.9 & 0.206 & 8.97 & 0.0196 \\
\hline PCT-NLS-15-a & 4 & 32.0 & 0.102 & 3.94 & 0.114 & 21.6 & 0.197 & 0.166 & 0.0494 & 0.0472 & 0.214 & 6.16 & 0.608 & 41.1 & 0.154 & 34.9 & 0.0762 \\
\hline PCT-NLS-15-b & 4 & 30.5 & 0.0975 & 3.86 & 0.112 & 21.2 & 0.193 & 0.170 & 0.0506 & 0.0445 & 0.202 & 6.06 & 0.599 & 39.5 & 0.148 & 32.9 & 0.0719 \\
\hline PCT-NLS-15-C & 4 & 32.2 & 0.103 & 3.95 & 0.114 & 20.6 & 0.188 & 0.174 & 0.0518 & 0.0467 & 0.211 & 6.26 & 0.618 & 41.4 & 0.155 & 34.1 & 0.0745 \\
\hline RWS-NLS-17-a & 8 & 0.401 & 0.00159 & 0.442 & 0.0159 & 94.2 & 1.068 & 0.0236 & 0.0087 & 0.361 & 2.03 & 0.280 & 0.0344 & 86.7 & 0.404 & 6.58 & 0.0179 \\
\hline RWS-NLS-17-b & 8 & 0.446 & 0.00143 & & & 109 & 0.991 & 0.0287 & 0.0086 & 0.268 & 1.22 & 0.282 & 0.0279 & 81.6 & 0.306 & 8.83 & 0.0193 \\
\hline RWS-NLS-17-C & 8 & 0.445 & 0.00143 & & & 117 & 1.07 & 0.0298 & 0.0089 & 0.253 & 1.15 & 0.301 & 0.0299 & 95.2 & 0.358 & 8.57 & 0.0188 \\
\hline PCT-NLS-17-a & 8 & 25.6 & 0.0818 & 1.77 & 0.0513 & 20.6 & 0.188 & 0.113 & 0.0337 & 0.0478 & 0.217 & 5.23 & 0.517 & 35.3 & 0.132 & 31.0 & 0.0677 \\
\hline PCT-NLS-17-b & 8 & 26.6 & 0.0849 & 1.68 & 0.0486 & 20.3 & 0.185 & 0.108 & 0.0321 & 0.0475 & 0.215 & 5.31 & 0.524 & 35.8 & 0.134 & 31.1 & 0.0679 \\
\hline PCT-NLS-17-C & 8 & 25.8 & 0.0829 & 1.69 & 0.0492 & 20.1 & 0.184 & 0.122 & 0.0365 & 0.0458 & 0.208 & 5.24 & 0.520 & 35.5 & 0.134 & 29.9 & 0.0656 \\
\hline RWS-NLS-26-a & 16 & 0.613 & 0.00201 & 0.352 & 0.0104 & 156 & 1.46 & 0.0272 & 0.0083 & 0.339 & 1.57 & 0.305 & 0.0308 & 106 & 0.407 & 7.20 & 0.0161 \\
\hline RWS-NLS-26-b & 16 & 0.420 & 0.00134 & & & 149 & 1.36 & 0.0246 & 0.0073 & 0.371 & 1.68 & 0.318 & 0.0315 & 102 & 0.383 & 7.55 & 0.0165 \\
\hline RWS-NLS-26-C & 16 & 0.392 & 0.00131 & & & 152 & 1.45 & 0.0254 & 0.0079 & 0.325 & 1.54 & 0.320 & 0.0331 & 101 & 0.396 & 7.54 & 0.0172 \\
\hline PCT-NLS-26-a & 16 & 27.9 & 0.0891 & 0.720 & 0.0209 & 23.8 & 0.217 & 0.0787 & 0.0234 & 0.0426 & 0.193 & 4.61 & 0.455 & 33.4 & 0.125 & 35.1 & 0.0765 \\
\hline PCT-NLS-26-b & 16 & 27.6 & 0.0880 & 0.949 & 0.0275 & 22.6 & 0.206 & 0.0946 & 0.0281 & 0.0433 & 0.196 & 4.57 & 0.452 & 31.9 & 0.120 & 35.1 & 0.0767 \\
\hline PCT-NLS-26-C & 16 & 27.2 & 0.0869 & 0.903 & 0.0262 & 23.4 & 0.213 & 0.0917 & 0.0273 & 0.0419 & 0.190 & 4.65 & 0.459 & 32.5 & 0.122 & 34.9 & 0.0761 \\
\hline RWS-NLS-24-a & 24 & 0.350 & 0.00112 & 0.236 & 0.0069 & 172 & 1.57 & 0.0246 & 0.0073 & 0.417 & 1.89 & 0.351 & 0.0347 & 115 & 0.432 & 7.51 & 0.0164 \\
\hline RWS-NLS-24-b & 24 & 0.536 & 0.00175 & & & 170 & 1.58 & 0.0275 & 0.0083 & 0.442 & 2.04 & 0.350 & 0.0353 & 115 & 0.440 & 7.17 & 0.0160 \\
\hline RWS-NLS-24-C & 24 & 0.382 & 0.00124 & & & 172 & 1.59 & 0.0263 & 0.0079 & 0.466 & 2.14 & 0.341 & 0.0341 & 114 & 0.433 & 7.21 & 0.0160 \\
\hline PCT-NLS-24-a & 24 & 25.3 & 0.0805 & 0.771 & 0.0223 & 25.4 & 0.231 & 0.0877 & 0.0260 & 0.0480 & 0.217 & 4.51 & 0.444 & 32.3 & 0.121 & 33.3 & 0.0726 \\
\hline PCT-NLS-24-b & 24 & 24.8 & 0.0793 & 0.882 & 0.0255 & 25.0 & 0.228 & 0.0967 & 0.0288 & 0.0491 & 0.222 & 4.52 & 0.446 & 31.5 & 0.118 & 33.6 & 0.0733 \\
\hline PCT-NLS-24-C & 24 & 26.7 & 0.0855 & 0.700 & 0.0203 & 24.7 & 0.226 & 0.0885 & 0.0264 & 0.0465 & 0.211 & 4.80 & 0.475 & 33.2 & 0.125 & 36.3 & 0.0794 \\
\hline RWS-NLS-27-a & 36 & 0.969 & 0.00321 & 0.268 & 0.0080 & 157 & 1.49 & 0.0230 & 0.0071 & 0.3680 & 1.73 & 0.335 & 0.0343 & 109 & 0.424 & 7.22 & 0.0164 \\
\hline RWS-NLS-27-b & 36 & 0.813 & 0.00271 & & & 161 & 1.53 & 0.0230 & 0.0072 & 0.379 & 1.79 & 0.340 & 0.0351 & 110 & 0.431 & 6.76 & 0.0154 \\
\hline RWS-NLS-27-C & 36 & 0.926 & 0.00315 & & & 174 & 1.69 & 0.0244 & 0.0077 & 0.368 & 1.77 & 0.349 & 0.0367 & 109 & 0.435 & 7.98 & 0.0186 \\
\hline PCT-NLS-27-a & 36 & 24.8 & 0.0793 & 0.820 & 0.0237 & 23.2 & 0.211 & 0.0798 & 0.0237 & 0.0389 & 0.176 & 4.64 & 0.458 & 30.2 & 0.113 & 33.7 & 0.0736 \\
\hline PCT-NLS-27-b & 36 & 24.1 & 0.0778 & 0.864 & 0.0253 & 21.9 & 0.202 & 0.0883 & 0.0266 & 0.0386 & 0.177 & 4.76 & 0.475 & 29.3 & 0.111 & 33.6 & 0.0741 \\
\hline PCT-NLS-27-C & 36 & 25.9 & 0.0826 & 0.758 & 0.0219 & 22.2 & 0.202 & 0.0777 & 0.0231 & 0.0392 & 0.177 & 5.01 & 0.495 & 31.9 & 0.120 & 34.5 & 0.0752 \\
\hline
\end{tabular}


Table 41. Results of Triplicate PCT with T-t PC CWF Materials Processed at $875^{\circ} \mathrm{C}$

\begin{tabular}{|c|c|c|c|c|c|c|c|c|c|c|c|c|c|c|c|c|c|}
\hline & $\begin{array}{c}\text { Time, } \\
\mathrm{h}\end{array}$ & $\begin{array}{c}\mathrm{Al}, \\
\mathrm{mg} / \mathrm{L}\end{array}$ & $\begin{array}{c}N L(\mathrm{Al}) \\
\mathrm{g} / \mathrm{m}^{2}\end{array}$ & $\begin{array}{c}\mathrm{B}, \\
\mathrm{mg} / \mathrm{L}\end{array}$ & $\begin{array}{l}N L(B) \\
g / m^{2}\end{array}$ & $\begin{array}{c}\mathrm{Cl}, \\
\mathrm{mg} / \mathrm{L}\end{array}$ & $\begin{array}{c}N L(\mathrm{Cl}) \\
\mathrm{g} / \mathrm{m}^{2}\end{array}$ & $\begin{array}{l}\mathrm{Cs}, \\
\mathrm{mg} / \mathrm{L}\end{array}$ & $\begin{array}{c}N L(\mathrm{Cs}) \\
\mathrm{g} / \mathrm{m}^{2}\end{array}$ & $\begin{array}{c}\mathrm{I}, \\
\mathrm{mg} / \mathrm{L}\end{array}$ & $\begin{array}{l}N L(\mathrm{I}) \\
\mathrm{g} / \mathrm{m}^{2}\end{array}$ & $\begin{array}{c}\mathrm{Li}, \\
\mathrm{mg} / \mathrm{L}\end{array}$ & $\begin{array}{c}N L(\mathrm{Li}) \\
\mathrm{g} / \mathrm{m}^{2}\end{array}$ & $\begin{array}{l}\mathrm{Na}, \\
\mathrm{mg} / \mathrm{L}\end{array}$ & $\begin{array}{c}N L(\mathrm{Na}) \\
\mathrm{g} / \mathrm{m}^{2}\end{array}$ & $\begin{array}{c}\mathrm{Si}, \\
\mathrm{mg} / \mathrm{L}\end{array}$ & $\begin{array}{c}N L(\mathrm{Si}) \\
\mathrm{g} / \mathrm{m}^{2}\end{array}$ \\
\hline RWS-NLS-3-a & 1 & 1.15 & 0.00368 & 1.38 & 0.0401 & 13.4 & 0.122 & 0.0504 & 0.0150 & 0.0317 & 0.144 & 0.090 & 0.0089 & 10.0 & 0.0370 & 9.61 & 0.0210 \\
\hline RWS-NLS-3-b & 1 & 1.36 & 0.00436 & & & 16.5 & 0.150 & 0.0534 & 0.0159 & 0.0382 & 0.173 & 0.099 & 0.0098 & 11.2 & 0.0420 & 9.53 & 0.0209 \\
\hline RWS-NLS-3-c & 1 & 1.71 & 0.00547 & & & 16.1 & 0.147 & 0.0617 & 0.0184 & 0.0418 & 0.190 & 0.110 & 0.0109 & 12.0 & 0.0450 & 9.97 & 0.0218 \\
\hline PCT-NLS-3-a & 1 & 32.7 & 0.104 & 15.5 & 0.448 & 22.6 & 0.205 & 0.173 & 0.0514 & 0.0482 & 0.218 & 5.41 & 0.533 & 45.7 & 0.171 & 32.7 & 0.0712 \\
\hline PCT-NLS-3-b & 1 & 32.9 & 0.104 & 16.9 & 0.487 & 21.1 & 0.191 & 0.157 & 0.0464 & 0.0475 & 0.214 & 5.56 & 0.547 & 46.2 & 0.172 & 32.4 & 0.0703 \\
\hline PCT-NLS-3-C & 1 & 32.2 & 0.101 & 16.4 & 0.468 & 22.3 & 0.200 & 0.143 & 0.0419 & 0.0438 & 0.196 & 5.58 & 0.544 & 45.3 & 0.167 & 31.8 & 0.0684 \\
\hline RWS-NLS-14-a & 4 & 0.365 & 0.00117 & 0.462 & 0.0134 & 111 & 1.02 & 0.0263 & 0.0078 & 0.227 & 1.03 & & & 89.0 & 0.334 & 8.08 & 0.0177 \\
\hline RWS-NLS-14-b & 4 & 0.388 & 0.00125 & & & 132 & 1.21 & 0.0283 & 0.0085 & 0.243 & 1.11 & & & 102 & 0.384 & 8.17 & 0.0180 \\
\hline RWS-NLS-14-C & 4 & 0.360 & 0.00107 & & & 118 & 1.00 & 0.0271 & 0.0075 & 0.216 & 0.910 & & & 87.8 & 0.306 & 9.44 & 0.0192 \\
\hline PCT-NLS-14-a & 4 & 25.8 & 0.0836 & 3.18 & 0.0934 & 27.3 & 0.252 & 0.133 & 0.0401 & 0.0562 & 0.258 & 4.88 & 0.489 & 35.9 & 0.136 & 30.7 & 0.0680 \\
\hline PCT-NLS-14-b & 4 & 28.6 & 0.0913 & 3.31 & 0.0958 & 27.8 & 0.253 & 0.125 & 0.0372 & 0.0593 & 0.268 & 4.99 & 0.493 & 35.8 & 0.134 & 32.8 & 0.0716 \\
\hline PCT-NLS-14-C & 4 & 25.2 & 0.0805 & 3.39 & 0.0981 & 27.9 & 0.254 & 0.142 & 0.0422 & 0.0547 & 0.247 & 5.00 & 0.493 & 34.4 & 0.129 & 30.8 & 0.0672 \\
\hline RWS-NLS-19-a & 8 & 0.412 & 0.00132 & 0.285 & 0.0083 & 206 & 1.88 & 0.0236 & 0.0070 & 0.360 & 1.63 & 0.397 & 0.0392 & 129 & 0.483 & 8.91 & 0.0195 \\
\hline RWS-NLS-19-b & 8 & 0.286 & 0.00091 & & & 188 & 1.71 & 0.0201 & 0.0060 & 0.336 & 1.52 & 0.370 & 0.0365 & 115 & 0.430 & 8.29 & 0.0181 \\
\hline RWS-NLS-19-C & 8 & 0.277 & 0.00089 & & & 178 & 1.62 & 0.0204 & 0.0061 & 0.297 & 1.35 & 0.376 & 0.0371 & 118 & 0.442 & 8.26 & 0.0181 \\
\hline PCT-NLS-19-a & 8 & 22.5 & 0.0718 & 1.59 & 0.0458 & 44.2 & 0.402 & 0.140 & 0.0417 & 0.0916 & 0.414 & 6.26 & 0.617 & 44.2 & 0.165 & 30.1 & 0.0656 \\
\hline PCT-NLS-19-b & 8 & 21.8 & 0.0705 & 1.35 & 0.0397 & 40.0 & 0.369 & 0.127 & 0.0383 & 0.0816 & 0.374 & 5.77 & 0.576 & 42.0 & 0.159 & 27.6 & 0.0609 \\
\hline PCT-NLS-19-c & 8 & 21.6 & 0.0689 & 1.29 & 0.0372 & 41.3 & 0.376 & 0.115 & 0.0343 & 0.0890 & 0.403 & 5.67 & 0.560 & 42.0 & 0.157 & 27.6 & 0.0603 \\
\hline RWS-NLS-28-a & 16 & 0.556 & 0.00178 & 0.231 & 0.0067 & 215 & 1.96 & 0.0470 & 0.0140 & 0.550 & 2.49 & 0.342 & 0.0338 & 126 & 0.474 & 6.08 & 0.0133 \\
\hline RWS-NLS-28-b & 16 & 0.766 & 0.00250 & & & 249 & 2.31 & 0.0530 & 0.0161 & 0.605 & 2.80 & 0.352 & 0.0355 & 151 & 0.576 & 6.98 & 0.0156 \\
\hline RWS-NLS-28-C & 16 & 0.912 & 0.00295 & & & 238 & 2.19 & 0.0537 & 0.0162 & 0.554 & 2.54 & 0.368 & 0.0368 & 143 & 0.541 & 6.65 & 0.0147 \\
\hline PCT-NLS-28-a & 16 & 19.3 & 0.0618 & 1.14 & 0.0331 & 18.9 & 0.172 & 0.142 & 0.0422 & 0.0419 & 0.190 & 4.45 & 0.440 & 25.7 & 0.0963 & 29.7 & 0.0650 \\
\hline PCT-NLS-28-b & 16 & 18.7 & 0.0598 & 1.14 & 0.0331 & 19.5 & 0.178 & 0.147 & 0.0439 & 0.0408 & 0.185 & 4.43 & 0.438 & 25.2 & 0.0947 & 29.2 & 0.0640 \\
\hline PCT-NLS-28-C & 16 & 18.7 & 0.0598 & 1.13 & 0.0327 & 18.5 & 0.169 & 0.141 & 0.0420 & 0.0415 & 0.188 & 4.59 & 0.453 & 26.1 & 0.0977 & 29.4 & 0.0642 \\
\hline RWS-NLS-33-a & 24 & 0.726 & 0.00232 & 0.250 & 0.0073 & 233 & 2.12 & 0.0323 & 0.0096 & 0.581 & 2.63 & 0.324 & 0.0320 & 141 & 0.530 & 8.34 & 0.0182 \\
\hline RWS-NLS-33-b & 24 & 0.473 & 0.00151 & & & 234 & 2.13 & 0.0306 & 0.0091 & 0.539 & 2.44 & 0.294 & 0.0291 & 146 & 0.546 & 7.53 & 0.0165 \\
\hline RWS-NLS-33-C & 24 & 0.817 & 0.00259 & & & 238 & 2.15 & 0.0340 & 0.0101 & 0.574 & 2.58 & 0.321 & 0.0315 & 141 & 0.526 & 8.51 & 0.0185 \\
\hline PCT-NLS-33-a & 24 & 19.5 & 0.0625 & 1.15 & 0.0335 & 18.8 & 0.172 & 0.0979 & 0.0292 & 0.0465 & 0.211 & 3.69 & 0.365 & 27.6 & 0.104 & 29.9 & 0.0655 \\
\hline PCT-NLS-33-b & 24 & 20.3 & 0.0660 & 1.14 & 0.0337 & 19.1 & 0.177 & 0.101 & 0.0305 & 0.0434 & 0.200 & 3.90 & 0.392 & 28.2 & 0.108 & 30.7 & 0.0683 \\
\hline PCT-NLS-33-c & 24 & 20.1 & 0.0642 & 1.06 & 0.0307 & 18.0 & 0.164 & 0.0971 & 0.0289 & 0.0442 & 0.200 & 4.14 & 0.409 & 27.7 & 0.104 & 30.9 & 0.0674 \\
\hline RWS-NLS-23-a & 36 & 0.438 & 0.00145 & 0.184 & 0.0055 & 167 & 1.57 & 0.0233 & 0.0072 & 0.463 & 2.17 & 0.339 & 0.0348 & 109 & 0.424 & 7.42 & 0.0168 \\
\hline RWS-NLS-23-b & 36 & 0.416 & 0.00139 & & & 183 & 1.74 & 0.0240 & 0.0075 & 0.453 & 2.14 & 0.360 & 0.0371 & 129 & 0.506 & 6.74 & 0.0154 \\
\hline RWS-NLS-23-c & 36 & 0.585 & 0.00199 & & & 180 & 1.74 & 0.0248 & 0.0079 & 0.436 & 2.10 & 0.377 & 0.0396 & 121 & 0.483 & 7.12 & 0.0166 \\
\hline PCT-NLS-23-a & 36 & 28.6 & 0.0914 & 0.696 & 0.0202 & 27.4 & 0.249 & 0.0838 & 0.0249 & 0.0531 & 0.240 & 4.37 & 0.431 & 35.4 & 0.132 & 39.0 & 0.0850 \\
\hline PCT-NLS-23-b & 36 & 24.1 & 0.0783 & 0.701 & 0.0206 & 25.7 & 0.237 & 0.0810 & 0.0245 & 0.0501 & 0.230 & 4.24 & 0.425 & 30.8 & 0.117 & 33.0 & 0.0732 \\
\hline PCT-NLS-23-C & 36 & 24.0 & 0.0767 & 0.746 & 0.0216 & 24.1 & 0.219 & 0.0891 & 0.0265 & 0.0490 & 0.222 & 4.57 & 0.451 & 31.3 & 0.117 & 34.0 & 0.0742 \\
\hline
\end{tabular}


Table 42. Results of Triplicate PCT with T-t PC CWF Materials Processed at $900^{\circ} \mathrm{C}$

\begin{tabular}{|c|c|c|c|c|c|c|c|c|c|c|c|c|c|c|c|c|c|}
\hline & $\begin{array}{c}\text { Time, } \\
\mathrm{h}\end{array}$ & $\begin{array}{c}\mathrm{Al}, \\
\mathrm{mg} / \mathrm{L}\end{array}$ & $\begin{array}{c}N L(\mathrm{Al}) \\
\mathrm{g} / \mathrm{m}^{2}\end{array}$ & $\begin{array}{c}\mathrm{B}, \\
\mathrm{mg} / \mathrm{L}\end{array}$ & $\begin{array}{l}N L(\mathrm{~B}) \\
\mathrm{g} / \mathrm{m}^{2}\end{array}$ & $\begin{array}{c}\mathrm{Cl}, \\
\mathrm{mg} / \mathrm{L}\end{array}$ & $\begin{array}{c}N L(\mathrm{Cl}) \\
\mathrm{g} / \mathrm{m}^{2}\end{array}$ & $\begin{array}{l}\mathrm{Cs}, \\
\mathrm{mg} / \mathrm{L}\end{array}$ & $\begin{array}{c}N L(C s) \\
g / m^{2}\end{array}$ & $\begin{array}{c}\mathrm{I} \\
\mathrm{mg} / \mathrm{L}\end{array}$ & $\begin{array}{l}\mathrm{NL}(\mathrm{I}) \\
\mathrm{g} / \mathrm{m}^{2}\end{array}$ & $\begin{array}{c}\mathrm{Li}, \\
\mathrm{mg} / \mathrm{L}\end{array}$ & $\begin{array}{l}N L(\mathrm{Li}) \\
\mathrm{g} / \mathrm{m}^{2}\end{array}$ & $\begin{array}{c}\mathrm{Na}, \\
\mathrm{mg} / \mathrm{L}\end{array}$ & $\begin{array}{c}N L(\mathrm{Na}) \\
\mathrm{g} / \mathrm{m}^{2}\end{array}$ & $\begin{array}{c}\mathrm{Si}, \\
\mathrm{mg} / \mathrm{L}\end{array}$ & $\begin{array}{c}N L(\mathrm{Si}) \\
\mathrm{g} / \mathrm{m}^{2}\end{array}$ \\
\hline RWS-NLS-4-a & 1 & 1.16 & 0.00372 & 1.11 & 0.0322 & 64.4 & 0.587 & 0.0385 & 0.0115 & 0.132 & 0.598 & 0.106 & 0.0105 & 40.0 & 0.150 & 9.14 & 0.0200 \\
\hline RWS-NLS-4-b & 1 & 1.20 & 0.00386 & & & 62.5 & 0.573 & 0.0376 & 0.0112 & 0.124 & 0.564 & 0.109 & 0.0108 & 37.1 & 0.140 & 9.13 & 0.0200 \\
\hline RWS-NLS-4-c & 1 & 1.26 & 0.00404 & & & 66.0 & 0.602 & 0.0351 & 0.0105 & 0.136 & 0.616 & 0.116 & 0.0115 & 39.1 & 0.147 & 8.86 & 0.0194 \\
\hline P-NLS-4-a & 1 & 29.2 & 0.0931 & 6.62 & 0.192 & 19.5 & 0.177 & 0.152 & 0.0453 & 0.035 & 0.159 & 4.80 & 0.474 & 36.1 & 0.135 & 32.0 & 0.0698 \\
\hline P-NLS-4-b & 1 & 29.1 & 0.0931 & 6.23 & 0.181 & 20.0 & 0.182 & 0.149 & 0.0443 & 0.050 & 0.227 & 4.66 & 0.461 & 35.9 & 0.135 & 31.2 & 0.0680 \\
\hline P-NLS-4-C & 1 & 30.1 & 0.0962 & 6.20 & 0.180 & 20.4 & 0.186 & 0.145 & 0.0432 & 0.047 & 0.214 & 4.69 & 0.463 & 36.8 & 0.138 & 33.2 & 0.0724 \\
\hline RWS-NLS-10-a & 4 & 0.228 & 0.00073 & 0.320 & 0.0093 & 227 & 2.07 & 0.0250 & 0.0075 & 0.666 & 3.02 & 0.298 & 0.0295 & 161 & 0.603 & 7.87 & 0.0172 \\
\hline RWS-NLS-10-b & 4 & 0.416 & 0.00135 & & & 268 & 2.48 & 0.0286 & 0.0086 & 1.04 & 4.78 & 0.313 & 0.0314 & 171 & 0.649 & 7.92 & 0.0175 \\
\hline RWS-NLS-10-c & 4 & 0.250 & 0.00080 & & & 226 & 2.06 & 0.0260 & 0.0078 & 1.38 & 6.27 & 0.294 & 0.0291 & 144. & 0.543 & 8.27 & 0.0181 \\
\hline P-NLS-10-a & 4 & 23.6 & 0.0760 & 1.82 & 0.0531 & 38.1 & 0.350 & 0.142 & 0.0426 & 0.073 & 0.333 & 6.04 & 0.601 & 40.4 & 0.153 & 31.6 & 0.0695 \\
\hline P-NLS-10-b & 4 & 23.6 & 0.0755 & 1.77 & 0.0513 & 38.0 & 0.346 & 0.166 & 0.0494 & 0.074 & 0.335 & 5.98 & 0.591 & 39.9 & 0.150 & 31.4 & 0.0686 \\
\hline P-NLS-10-C & 4 & 22.2 & 0.0710 & 1.67 & 0.0484 & 38.5 & 0.351 & 0.166 & 0.0495 & 0.073 & 0.332 & 5.88 & 0.581 & 39.0 & 0.146 & 29.6 & 0.0647 \\
\hline RWS-NLS-20-a & 8 & 0.191 & 0.00061 & 0.215 & 0.0062 & 305 & 2.78 & 0.0225 & 0.0067 & 0.482 & 2.18 & 0.446 & 0.0441 & 159 & 0.597 & 10.8 & 0.0236 \\
\hline RWS-NLS-20-b & 8 & 0.252 & 0.00080 & & & 312 & 2.84 & 0.0246 & 0.0073 & 0.524 & 2.37 & 0.451 & 0.0445 & 168 & 0.630 & 9.66 & 0.0211 \\
\hline RWS-NLS-20-c & 8 & 0.192 & 0.00062 & & & 315 & 2.87 & 0.0239 & 0.0071 & 0.535 & 2.43 & 0.426 & 0.0422 & 171 & 0.643 & 8.81 & 0.0193 \\
\hline P-NLS-20-a & 8 & 18.7 & 0.0599 & 1.60 & 0.0464 & 53.5 & 0.488 & 0.174 & 0.0519 & 0.120 & 0.543 & 6.25 & 0.618 & 44.9 & 0.168 & 27.4 & 0.0598 \\
\hline P-NLS-20-b & 8 & 19.2 & 0.0623 & 1.61 & 0.0473 & 50.7 & 0.469 & 0.166 & 0.0501 & 0.114 & 0.524 & 6.20 & 0.622 & 43.8 & 0.166 & 27.8 & 0.0616 \\
\hline P-NLS-20-C & 8 & 19.5 & 0.0626 & 1.56 & 0.0452 & 51.2 & 0.468 & 0.197 & 0.0589 & 0.123 & 0.559 & 6.32 & 0.627 & 44.8 & 0.169 & 28.3 & 0.0619 \\
\hline RWS-NLS-29-a & 16 & 0.657 & 0.00225 & 0.232 & 0.0072 & 267 & 2.61 & 0.0340 & 0.0109 & 0.593 & 2.88 & 0.393 & 0.0416 & 160 & 0.645 & 7.02 & 0.0164 \\
\hline RWS-NLS-29-b & 16 & 0.582 & 0.00203 & & & 266 & 2.64 & 0.0316 & 0.0103 & 0.606 & 2.99 & 0.356 & 0.0383 & 154 & 0.628 & 7.01 & 0.0167 \\
\hline RWS-NLS-29-C & 16 & 0.651 & 0.00212 & & & 287 & 2.66 & 0.0328 & 0.0099 & 0.522 & 2.40 & 0.387 & 0.0389 & 167 & 0.635 & 7.35 & 0.0163 \\
\hline P-NLS-29-a & 16 & 31.5 & 0.101 & 1.47 & 0.0425 & 22.4 & 0.204 & 0.157 & 0.0467 & 0.045 & 0.203 & 6.04 & 0.597 & 40.7 & 0.152 & 48.3 & 0.105 \\
\hline P-NLS-29-b & 16 & 23.2 & 0.0741 & 1.06 & 0.0307 & 22.6 & 0.206 & 0.118 & 0.0351 & 0.046 & 0.207 & 4.73 & 0.467 & 30.3 & 0.113 & 33.7 & 0.0736 \\
\hline P-NLS-29-C & 16 & 20.5 & 0.0656 & 1.07 & 0.0311 & 20.7 & 0.189 & 0.117 & 0.0349 & 0.044 & 0.201 & 4.61 & 0.455 & 28.0 & 0.105 & 31.5 & 0.0689 \\
\hline RWS-NLS-34-a & 24 & 0.769 & 0.00246 & 0.243 & 0.0071 & 288 & 2.63 & 0.0378 & 0.0113 & 0.694 & 3.15 & 0.300 & 0.0297 & 177 & 0.664 & 5.77 & 0.0126 \\
\hline RWS-NLS-34-b & 24 & 0.921 & 0.00300 & & & 292 & 2.71 & 0.0423 & 0.0128 & 0.704 & 3.25 & 0.332 & 0.0334 & 184 & 0.703 & 7.42 & 0.0165 \\
\hline RWS-NLS-34-C & 24 & 0.686 & 0.00233 & & & 277 & 2.68 & 0.0365 & 0.0115 & 0.694 & 3.34 & 0.296 & 0.0311 & 174 & 0.693 & 6.78 & 0.0157 \\
\hline P-NLS-34-a & 24 & 21.1 & 0.0676 & 1.04 & 0.0302 & 20.8 & 0.190 & 0.106 & 0.0316 & 0.040 & 0.180 & 4.19 & 0.415 & 29.1 & 0.109 & 31.6 & 0.0691 \\
\hline P-NLS-34-b & 24 & 21.5 & 0.0689 & 1.07 & 0.0311 & 20.0 & 0.183 & 0.102 & 0.0304 & 0.041 & 0.187 & 3.90 & 0.386 & 29.9 & 0.112 & 31.8 & 0.0696 \\
\hline P-NLS-34-C & 24 & 22.0 & 0.0703 & 1.07 & 0.0311 & 21.4 & 0.195 & 0.101 & 0.0302 & 0.043 & 0.196 & 4.05 & 0.400 & 30.2 & 0.113 & 32.2 & 0.0704 \\
\hline RWS-NLS-18-a & 36 & 0.192 & 0.00062 & 0.182 & 0.0053 & 257 & 2.35 & 0.0231 & 0.0069 & 0.445 & 2.02 & 0.422 & 0.0417 & 145 & 0.544 & 7.67 & 0.0168 \\
\hline RWS-NLS-18-b & 36 & 0.300 & 0.00096 & & & 275 & 2.51 & 0.0251 & 0.0075 & 0.473 & 2.14 & 0.416 & 0.0411 & 144 & 0.540 & 9.26 & 0.0202 \\
\hline RWS-NLS-18-C & 36 & 0.206 & 0.00066 & & & 254 & 2.32 & 0.0231 & 0.0069 & 0.453 & 2.05 & 0.398 & 0.0394 & 142 & 0.533 & 8.30 & 0.0181 \\
\hline P-NLS-18-a & 36 & 21.2 & 0.0681 & 1.50 & 0.0435 & 35.3 & 0.323 & 0.158 & 0.0471 & 0.079 & 0.359 & 5.73 & 0.569 & 36.8 & 0.138 & 30.4 & 0.0667 \\
\hline P-NLS-18-b & 36 & 21.712 & 0.06983 & 1.555 & 0.0453 & 34.1 & 0.313 & 0.1394 & 0.0417 & 0.077 & 0.352 & 5.867 & 0.5832 & 37.57 & 0.142 & 31.41 & 0.0690 \\
\hline P-NLS-18-C & 36 & 23.231 & 0.07442 & 1.532 & 0.0445 & 35.0 & 0.320 & 0.1278 & 0.0381 & 0.085 & 0.384 & 5.955 & 0.5896 & 38.95 & 0.146 & 32.36 & 0.0708 \\
\hline
\end{tabular}


Table 43. Results of Triplicate PCT with T-t PC CWF Materials Processed at $915^{\circ} \mathrm{C}$

\begin{tabular}{|c|c|c|c|c|c|c|c|c|c|c|c|c|c|c|c|c|c|}
\hline & $\begin{array}{c}\text { Time, } \\
\mathrm{h}\end{array}$ & $\begin{array}{c}\mathrm{Al}, \\
\mathrm{mg} / \mathrm{L}\end{array}$ & $\begin{array}{c}\mathrm{NL}(\mathrm{Al}), \\
\mathrm{g} / \mathrm{m}^{2}\end{array}$ & $\begin{array}{c}\mathrm{B}, \\
\mathrm{mg} / \mathrm{L}\end{array}$ & $\begin{array}{l}N L(\mathrm{~B}) \\
\mathrm{g} / \mathrm{m}^{2}\end{array}$ & $\begin{array}{c}\mathrm{Cl}, \\
\mathrm{mg} / \mathrm{L}\end{array}$ & $\begin{array}{l}\mathrm{NL}(\mathrm{Cl}) \\
\mathrm{g} / \mathrm{m}^{2}\end{array}$ & $\begin{array}{c}\mathrm{Cs}, \\
\mathrm{mg} / \mathrm{L}\end{array}$ & $\begin{array}{c}\mathrm{NL}(\mathrm{Cs}) \\
\mathrm{g} / \mathrm{m}^{2}\end{array}$ & $\begin{array}{c}\mathrm{I} \\
\mathrm{mg} / \mathrm{L}\end{array}$ & $\begin{array}{l}N L(\mathrm{l}) \\
\mathrm{g} / \mathrm{m}^{2}\end{array}$ & $\begin{array}{c}\mathrm{Li}, \\
\mathrm{mg} / \mathrm{L}\end{array}$ & $\begin{array}{l}N L(\mathrm{Li}) \\
\mathrm{g} / \mathrm{m}^{2}\end{array}$ & $\begin{array}{l}\mathrm{Na}, \\
\mathrm{mg} / \mathrm{L}\end{array}$ & $\begin{array}{c}N L(\mathrm{Na}) \\
\mathrm{g} / \mathrm{m}^{2}\end{array}$ & $\begin{array}{c}\mathrm{Si}, \\
\mathrm{mg} / \mathrm{L}\end{array}$ & $\begin{array}{c}N L(\mathrm{Si}) \\
\mathrm{g} / \mathrm{m}^{2}\end{array}$ \\
\hline RWS-NLS-1-a & 1 & 0.843 & 0.00270 & 0.717 & 0.0208 & 107 & 0.977 & 0.0297 & 0.0089 & 0.233 & 1.06 & 0.105 & 0.0104 & 59.4 & 0.223 & 3.37 & 0.0074 \\
\hline RWS-NLS-1-b & 1 & 0.707 & 0.00227 & & & 131 & 1.20 & 0.0289 & 0.0086 & 0.273 & 1.24 & 0.114 & 0.0113 & 66.5 & 0.250 & 6.15 & 0.0135 \\
\hline RWS-NLS-1-c & 1 & 0.731 & 0.00233 & & & 113 & 1.03 & 0.0297 & 0.0088 & 0.233 & 1.05 & 0.102 & 0.0101 & 59.9 & 0.224 & 4.98 & 0.0109 \\
\hline PCT-NLS-1-a & 1 & 28.6 & 0.0913 & 5.44 & 0.158 & 24.8 & 0.226 & 0.168 & 0.0499 & 0.060 & 0.272 & 4.74 & 0.467 & 37.8 & 0.141 & 33.2 & 0.0725 \\
\hline PCT-NLS-1-b & 1 & 25.3 & 0806 & 4.91 & 0.142 & 24.1 & 0.219 & 0.161 & 0.0477 & 0.058 & 0.264 & 4.47 & 0.440 & 33.7 & 0.126 & 29.5 & 0.0643 \\
\hline PCT-NLS-1-C & 1 & 26.2 & 0.0835 & 3.32 & 0.0960 & 22.0 & 0.200 & 0.160 & 0.0475 & 0.056 & 0.254 & 4.57 & 0.451 & 35.3 & 0.132 & 31.3 & 0.0682 \\
\hline RWS-NLS-13-a & 4 & 0.0559 & 0.00018 & 0.223 & 0.0065 & 299 & 2.74 & 0.0318 & 0.0095 & 0.531 & 2.41 & 0.346 & 0.0343 & 172 & 0.648 & 7.82 & 0.0171 \\
\hline RWS-NLS-13-b & 4 & 0.161 & 0.00052 & & & 306 & 2.79 & 0.0327 & 0.0098 & 0.570 & 2.58 & 0.333 & 0.0330 & 207 & 0.775 & 7.93 & 0.0173 \\
\hline RWS-NLS-13-C & 4 & 0.186 & 0.00060 & & & 321 & 2.94 & 0.0347 & 0.0104 & 0.623 & 2.84 & 0.360 & 0.0357 & 209 & 0.786 & 8.30 & 0.0182 \\
\hline PCT-NLS-13-a & 4 & 20.2 & 0645 & 88 & 0833 & 39.1 & 0.356 & 0.190 & 0.0565 & 0.080 & 0.359 & 5.32 & 0.525 & 35.8 & 0.134 & 29.0 & 0.0632 \\
\hline PCT-NLS-13-b & 4 & .2 & 0644 & 2.84 & 0.0821 & 38.1 & 0.346 & 176 & 23 & 0.080 & 0.360 & 14 & 507 & 35.1 & 131 & 28.8 & 0.0628 \\
\hline PCT-NLS-13-C & 4 & 9.2 & 0.0612 & 2.87 & 0.0830 & 37.8 & 0.344 & 0.183 & 0.0544 & 0.079 & 0.355 & 5.20 & 0.513 & 34.2 & 0.128 & 27.4 & 0.0597 \\
\hline RWS-NLS-21-a & 8 & 0.281 & 0.00090 & 0.235 & 0.0068 & 286 & 2.61 & 0.0288 & 0.0086 & 0.560 & 2.54 & 0.432 & 0.0428 & 186 & 0.697 & 7.62 & 0.0167 \\
\hline RWS-NLS-21-b & 8 & 0.260 & 0.00087 & & & 292 & 2.79 & 0.0267 & 0.0083 & 0.506 & 2.41 & 0.419 & 0.0435 & 174 & 0.685 & 6.65 & 0.0153 \\
\hline RWS-NLS-21-C & 8 & 0.315 & 0.00101 & & & 307 & 2.81 & 0.0318 & 0.0095 & 0.590 & 2.68 & 0.443 & 0.0440 & 193 & 0.726 & 8.38 & 0.0184 \\
\hline PCT-NLS-21-a & 8 & 20.7 & 0.06639 & 1.54 & 0.0446 & 52.2 & 0.476 & 0.158 & 0.0471 & 0.123 & 0.558 & 6.42 & 0.636 & 45.8 & 0.172 & 28.9 & 0.0633 \\
\hline PCT-NLS-21-b & 8 & 19.7 & 0.06613 & 1.39 & 0.0424 & 50.5 & 0.484 & 0.147 & 0.0461 & 0.120 & 0.571 & 6.03 & 0.626 & 44.7 & 0.176 & 27.7 & 0.0635 \\
\hline b-21-c & 8 & 8 & 0.06 & 1.46 & 0.0423 & 51.6 & 0.472 & 0.185 & & 0.119 & 0.541 & 6.40 & 0.635 & 45.7 & 0.172 & 27.7 & 0.0607 \\
\hline RWS-I & 16 & 0.352 & 0.00113 & 0.249 & 0.0073 & 295 & 2.70 & 0.0166 & 50 & 0.781 & 3.55 & 0.115 & 0.0114 & 157 & 0.589 & 6.31 & 0.0139 \\
\hline RWS-NLS-8-b & 16 & 273 & 0.00087 & & & 293 & 2.67 & 0.0164 & 0.0049 & 0.855 & 3.87 & 0.119 & 0.0118 & 161 & 0.601 & 7.72 & 0.0168 \\
\hline RWS-NLS-8-C & 16 & 0.433 & 0.00139 & & & 279 & 2.54 & 0.0176 & 0.0052 & 0.814 & 3.69 & 0.113 & 0.0112 & 154 & 0.576 & 6.99 & 0.0153 \\
\hline PCT-NLS-8-a & 16 & 25.3 & 0.0809 & 2.22 & 42 & 35.5 & 0.323 & 0.115 & 343 & 0.094 & 0.426 & 4.97 & 0.491 & 43.4 & 0.163 & 31.7 & 0.0692 \\
\hline PCT-NLS-8-b & 16 & 24.3 & 0.0773 & 2.16 & 0.0625 & 38.2 & 0.349 & 0.138 & 0.0411 & 0.106 & 0.480 & 4.76 & 0.470 & 42.5 & 0.159 & 32.1 & 0.0702 \\
\hline PCT-NLS-8-C & 16 & 24.3 & 0.0776 & 2.25 & 0.0653 & 32.0 & 0.292 & 0.140 & 0.0415 & 0.096 & 0.433 & 5.04 & 0.498 & 40.5 & 0.152 & 32.5 & 0.0711 \\
\hline RWS-NLS-36-a & 24 & 0.517 & 0.00165 & 0.215 & 0.0062 & 318 & 2.89 & 0.0320 & 0.0095 & 0.783 & 3.54 & 0.29 & 0.0286 & 198 & 0.742 & 7.14 & 0.0156 \\
\hline RWS-NLS-36-b & 24 & & 0.00171 & & & 326 & 2.98 & 0.0349 & & 0.707 & 3.21 & 0.289 & 0.0286 & 199 & 0.748 & 6.68 & 0.0146 \\
\hline RWS-NLS-36-C & 24 & 0.455 & 0.00145 & & & 322 & 2.93 & & & 0.738 & 3.34 & 0.277 & 0.0273 & 194 & .727 & 7.51 & 0.0164 \\
\hline PCT-NLS-36-a & 24 & 24.1 & 0.0771 & 1.11 & 0323 & 26.1 & 0.238 & 0.0936 & 79 & 0.069 & 0.313 & 4.37 & 0.432 & 35.0 & 0.131 & 34.6 & 0.0755 \\
\hline S-36-b & 24 & .0 & 704 & 1.19 & 0.0346 & 22.5 & 0.205 & 0.113 & 337 & 0.066 & 0.298 & 4.35 & 0.429 & 32.4 & 0.122 & 33.7 & 0.0735 \\
\hline S-36-c & 2 & .2 & 6677 & 1.13 & 0.0326 & 23.4 & 0.213 & 00 & 98 & 0.067 & 0.301 & 4.15 & 0.409 & 31.0 & 116 & 32.0 & 0.0697 \\
\hline RWS-NLS-16-a & 36 & 0749 & 0.00024 & 0.185 & 0.0054 & 382 & 3.48 & 0.0647 & 0.0193 & 0.775 & 3.51 & 0.362 & 0.0358 & 224 & 0.839 & 8.26 & 0.0180 \\
\hline RWS-NLS-16-b & 36 & 0.0895 & 0.00030 & & & 382 & 3.62 & 0.0693 & 0.0215 & 0.784 & 3.69 & 0.376 & 0.0386 & 232 & 0.903 & 8.28 & 0.0188 \\
\hline RWS-NLS-16-C & 36 & 0.112 & 0.00036 & & & 395 & 3.60 & 0.0699 & 0.0208 & 1.05 & 4.75 & 0.369 & 0.0364 & 222 & 0.831 & 9.09 & 0.0199 \\
\hline PCT-NLS-16-a & 36 & 18.9 & 0.0603 & 1.72 & 0.0498 & 30.6 & 0.278 & 0.206 & 0.0612 & 0.082 & 0.369 & 5.65 & 0.557 & 35.5 & 0.133 & 28.5 & 0.0622 \\
\hline PCT-NLS-16-b & 36 & 19.6 & 0.0626 & 1.70 & 0.0492 & 32.5 & 0.296 & 0.221 & 0.0657 & 0.083 & 0.376 & 5.72 & 0.565 & 35.4 & 0.133 & 29.3 & 0.0639 \\
\hline PCT-NLS-16-C & 36 & 21.0 & 0.0671 & 1.68 & 0.0487 & 30.7 & 0.280 & 0.192 & 0.0571 & 0.084 & 0.381 & 5.68 & 0.561 & 35.4 & 0.133 & 29.9 & 0.0653 \\
\hline
\end{tabular}


Table 44. Results of Triplicate PCT with T-t PC CWF Materials Processed at $925^{\circ} \mathrm{C}$

\begin{tabular}{|c|c|c|c|c|c|c|c|c|c|c|c|c|c|c|c|c|c|}
\hline & $\begin{array}{c}\text { Time, } \\
\mathrm{h}\end{array}$ & $\begin{array}{c}\mathrm{Al}, \\
\mathrm{mg} / \mathrm{L}\end{array}$ & $\begin{array}{c}N L(\mathrm{Al}), \\
\mathrm{g} / \mathrm{m}^{2}\end{array}$ & $\begin{array}{c}\mathrm{B}, \\
\mathrm{mg} / \mathrm{L}\end{array}$ & $\begin{array}{c}N L(B) \\
g / m^{2}\end{array}$ & $\begin{array}{c}\mathrm{Cl}, \\
\mathrm{mg} / \mathrm{L}\end{array}$ & $\begin{array}{c}\mathrm{NL}(\mathrm{Cl}) \\
\mathrm{g} / \mathrm{m}^{2}\end{array}$ & $\begin{array}{l}\mathrm{Cs}, \\
\mathrm{mg} / \mathrm{L}\end{array}$ & $\begin{array}{c}N L(\mathrm{Cs}) \\
\mathrm{g} / \mathrm{m}^{2}\end{array}$ & $\begin{array}{c}\mathrm{I}, \\
\mathrm{mg} / \mathrm{L}\end{array}$ & $\begin{array}{l}N L(\mathrm{I}) \\
\mathrm{g} / \mathrm{m}^{2}\end{array}$ & $\begin{array}{c}\mathrm{Li}, \\
\mathrm{mg} / \mathrm{L}\end{array}$ & $\begin{array}{c}N L(\mathrm{Li}) \\
\mathrm{g} / \mathrm{m}^{2}\end{array}$ & $\begin{array}{l}\mathrm{Na}, \\
\mathrm{mg} / \mathrm{L}\end{array}$ & $\begin{array}{c}N L(\mathrm{Na}) \\
\mathrm{g} / \mathrm{m}^{2}\end{array}$ & $\begin{array}{c}\mathrm{Si}, \\
\mathrm{mg} / \mathrm{L}\end{array}$ & $\begin{array}{c}N L(\mathrm{Si}) \\
\mathrm{g} / \mathrm{m}^{2}\end{array}$ \\
\hline RWS-NLS-5-a & 1 & 0.450 & 0.0014 & 0.524 & 0.0152 & 135 & 1.23 & 0.0208 & 0.00618 & 0.324 & 1.46 & 0.0995 & 0.0098 & 74.4 & 0.278 & 6.34 & 0.0138 \\
\hline RWS-NLS-5-b & 1 & 0.616 & 0.0020 & & & 153 & 1.39 & 0.0230 & 0.00683 & 0.241 & 1.09 & 0.111 & 0.0109 & 73.4 & 0.274 & 11.2 & 0.0244 \\
\hline RWS-NLS-5-c & 1 & 0.450 & 0.0015 & & & 121 & 1.15 & 0.0214 & 0.00664 & 0.204 & 0.962 & 0.0994 & 0.0102 & 66.6 & 0.260 & 6.19 & 0.0141 \\
\hline PCT-NLS-5-a & 1 & 24.4 & 0.0780 & 4.88 & 0.141 & 34.7 & 0.316 & 0.140 & 0.0416 & 0.081 & 0.365 & 4.71 & 0.465 & 41.7 & 0.156 & 27.7 & 0.0606 \\
\hline PCT-NLS-5-b & 1 & 25.4 & 0.0810 & 3.28 & 0.0948 & 31.5 & 0.287 & 0.133 & 0.0394 & 0.081 & 0.367 & 4.54 & 0.448 & 40.7 & 0.152 & 28.5 & 0.0622 \\
\hline PCT-NLS-5-C & 1 & 24.8 & 0.0792 & 2.86 & 0.0826 & 29.5 & 0.268 & 0.130 & 0.0385 & 0.070 & 0.315 & 4.41 & 0.434 & 40.4 & 0.151 & 28.5 & 0.0620 \\
\hline RWS-NLS-12-a & 4 & 0.116 & 0.0004 & 0.212 & 0.0062 & 316 & 2.88 & 0.0396 & 0.0118 & 0.617 & 2.80 & 0.349 & 0.0346 & 202 & 0.759 & 6.99 & 0.0153 \\
\hline RWS-NLS-12-b & 4 & 0.097 & 0.0003 & & & 340 & 3.10 & 0.0434 & 0.0129 & 0.674 & 3.05 & 0.358 & 0.0354 & 221 & 0.830 & 7.86 & 0.0172 \\
\hline RWS-NLS-12-C & 4 & 0.044 & 0.0001 & & & 281 & 2.56 & 0.0381 & 0.0113 & 0.542 & 2.46 & 0.326 & 0.0322 & 184 & 0.691 & 7.44 & 0.0163 \\
\hline PCT-NLS-12-a & 4 & 15.7 & 0.0506 & 2.79 & 0.0815 & 64.7 & 0.595 & 0.211 & 0.0633 & 0.108 & 0.493 & 4.93 & 0.491 & 42.8 & 0.162 & 22.5 & 0.0495 \\
\hline PCT-NLS-12-b & 4 & 15.5 & 0.0496 & 3.09 & 0.0896 & 64.2 & 0.585 & 0.210 & 0.0625 & 0.114 & 0.516 & 5.84 & 0.577 & 43.4 & 0.163 & 22.1 & 0.0483 \\
\hline PCT-NLS-12-C & 4 & 16.6 & 0.0531 & 2.99 & 0.0867 & 63.1 & 0.575 & 0.222 & 0.0661 & 0.114 & 0.516 & 5.34 & 0.528 & 45.3 & 0.170 & 24.6 & 0.0537 \\
\hline RWS-NLS-22-a & 8 & 0.323 & 0.0011 & 0.208 & 0.0062 & 342 & 3.19 & 0.0381 & 0.0116 & 0.737 & 3.42 & 0.468 & 0.0474 & 219 & 0.841 & 5.27 & 0.0118 \\
\hline RWS-NLS-22-b & 8 & 0.340 & 0.0011 & & & 368 & 3.36 & 0.0390 & 0.0116 & 0.784 & 3.55 & 0.430 & 0.0425 & 219 & 0.823 & 6.32 & 0.0138 \\
\hline RWS-NLS-22-c & 8 & 0.294 & 0.0010 & & & 365 & 3.47 & 0.0376 & 0.0117 & 0.840 & 3.97 & 0.447 & 0.0461 & 230 & 0.898 & 6.59 & 0.0150 \\
\hline PCT-NLS-22-a & 8 & 18.6 & 0.0598 & 1.14 & 0.0332 & 40.6 & 0.373 & 0.161 & 0.0484 & 0.091 & 0.415 & 4.82 & 0.480 & 34.1 & 0.129 & 30.3 & 0.0666 \\
\hline PCT-NLS-22-b & 8 & 21.0 & 0.0672 & 1.15 & 0.0332 & 39.9 & 0.363 & 0.170 & 0.0508 & 0.088 & 0.400 & 5.29 & 0.523 & 37.4 & 0.140 & 35.1 & 0.0767 \\
\hline PCT-NLS-22-c & 8 & 18.7 & 0.0597 & 1.09 & 0.0316 & 38.9 & 0.355 & 0.167 & 0.0499 & 0.089 & 0.404 & 5.18 & 0.513 & 34.3 & 0.129 & 31.2 & 0.0681 \\
\hline RWS-NLS-30-a & 16 & 0.267 & 0.0009 & 0.192 & 0.0056 & 337 & 3.10 & 0.0404 & 0.0122 & 0.705 & 3.23 & 0.36 & 0.0361 & 200 & 0.757 & 6.76 & 0.0149 \\
\hline RWS-NLS-30-b & 16 & 0.362 & 0.0012 & & & 352 & 3.22 & 0.0423 & 0.0126 & 0.747 & 3.39 & 0.377 & 0.0373 & 189 & 0.710 & 7.20 & 0.0158 \\
\hline RWS-NLS-30-c & 16 & 0.331 & 0.0011 & & & 360 & 3.27 & 0.0416 & 0.0124 & 0.746 & 3.37 & 0.366 & 0.0361 & 205 & 0.768 & 8.83 & 0.0192 \\
\hline PCT-NLS-30-a & 16 & 20.5 & 0.0655 & 1.56 & 0.0451 & 34.3 & 0.312 & 0.125 & 0.0373 & 0.069 & 0.314 & 4.25 & 0.420 & 36.1 & 0.135 & 33.4 & 0.0728 \\
\hline PCT-NLS-30-b & 16 & 19.0 & 0.0607 & 1.59 & 0.0462 & 34.2 & 0.312 & 0.128 & 0.0382 & 0.068 & 0.309 & 4.22 & 0.417 & 33.5 & 0.126 & 30.8 & 0.0673 \\
\hline PCT-NLS-30-c & 16 & 21.4 & 0.0686 & 1.54 & 0.0449 & 32.8 & 0.300 & 0.135 & 0.0404 & 0.070 & 0.318 & 4.20 & 0.416 & 36.2 & 0.136 & 35.2 & 0.0771 \\
\hline RWS-NLS-35-a & 24 & 0.393 & 0.0013 & 0.207 & 0.0063 & 399 & 3.82 & 0.0404 & 0.0127 & 0.819 & 3.90 & 0.258 & 0.0268 & 232 & 0.916 & 6.98 & 0.0160 \\
\hline RWS-NLS-35-b & 24 & 0.399 & 0.0013 & & & 437 & 3.99 & 0.0419 & 0.0125 & 0.853 & 3.87 & 0.276 & 0.0273 & 261 & 0.978 & 7.38 & 0.0162 \\
\hline RWS-NLS-35-C & 24 & 0.488 & 0.0016 & & & 431 & 3.93 & 0.0426 & 0.0127 & 0.863 & 3.91 & 0.265 & 0.0262 & 241 & 0.905 & 7.07 & 0.0155 \\
\hline PCT-NLS-35-a & 24 & 21.1 & 0.0681 & 1.15 & 0.0337 & 29.3 & 0.270 & 0.124 & 0.0374 & 0.055 & 0.250 & 4.32 & 0.431 & 33.0 & 0.125 & 32.5 & 0.0717 \\
\hline PCT-NLS-35-b & 24 & 22.5 & 0.0724 & 1.17 & 0.0341 & 28.8 & 0.264 & 0.116 & 0.0348 & 0.054 & 0.246 & 4.34 & 0.431 & 34.3 & 0.129 & 33.9 & 0.0745 \\
\hline PCT-NLS-35-c & 24 & 22.3 & 0.0712 & 1.16 & 0.0335 & 28.7 & 0.261 & 0.123 & 0.0365 & 0.057 & 0.259 & 4.38 & 0.432 & 33.8 & 0.127 & 33.7 & 0.0737 \\
\hline RWS-NLS-11-a & 36 & 0.044 & 0.0001 & 0.177 & 0.0052 & 395 & 3.62 & 0.0478 & 0.0143 & 0.769 & 3.50 & 0.406 & 0.0403 & 238 & 0.896 & 7.40 & 0.0162 \\
\hline RWS-NLS-11-b & 36 & 0.027 & 0.0001 & & & 348 & 3.18 & 0.0433 & 0.0129 & 0.649 & 2.94 & 0.375 & 0.0371 & 201 & 0.753 & 7.52 & 0.0165 \\
\hline RWS-NLS-11-c & 36 & 0.134 & 0.0004 & & & 384 & 3.50 & 0.0481 & 0.0143 & 0.694 & 3.15 & 0.404 & 0.0400 & 229 & 0.858 & 7.37 & 0.0161 \\
\hline PCT-NLS-11-a & 36 & 17.2 & 0.0551 & 1.86 & 0.0541 & 78.6 & 0.719 & 0.182 & 0.0543 & 0.131 & 0.595 & 5.34 & 0.529 & 54.8 & 0.206 & 25.7 & 0.0563 \\
\hline PCT-NLS-11-b & 36 & 16.3 & 0.0533 & 1.80 & 0.0534 & 75.7 & 0.706 & 0.186 & 0.0567 & 0.132 & 0.612 & 5.42 & 0.548 & 53.9 & 0.207 & 25.0 & 0.0559 \\
\hline PCT-NLS-11-C & 36 & 17.7 & 0.0566 & 1.82 & 0.0528 & 76.3 & 0.696 & 0.177 & 0.0527 & 0.138 & 0.625 & 5.31 & 0.525 & 55.1 & 0.207 & 26.3 & 0.0575 \\
\hline
\end{tabular}


Table 45. Results of Triplicate PCT with T-t PC CWF Materials Processed at $950^{\circ} \mathrm{C}$

\begin{tabular}{|c|c|c|c|c|c|c|c|c|c|c|c|c|c|c|c|c|c|}
\hline & $\begin{array}{c}\text { Time, } \\
\mathrm{h}\end{array}$ & $\begin{array}{c}\mathrm{Al}, \\
\mathrm{mg} / \mathrm{L}\end{array}$ & $\begin{array}{c}N L(\mathrm{Al}) \\
\mathrm{g} / \mathrm{m}^{2}\end{array}$ & $\begin{array}{c}\mathrm{B}, \\
\mathrm{mg} / \mathrm{L}\end{array}$ & $\begin{array}{l}N L(B) \\
g / m^{2}\end{array}$ & $\begin{array}{c}\mathrm{Cl}, \\
\mathrm{mg} / \mathrm{L}\end{array}$ & $\begin{array}{c}N L(\mathrm{Cl}) \\
\mathrm{g} / \mathrm{m}^{2}\end{array}$ & $\begin{array}{c}\mathrm{Cs}, \\
\mathrm{mg} / \mathrm{L}\end{array}$ & $\begin{array}{c}N L(\mathrm{Cs}) \\
\mathrm{g} / \mathrm{m}^{2}\end{array}$ & $\begin{array}{c}\mathrm{I}, \\
\mathrm{mg} / \mathrm{L}\end{array}$ & $\begin{array}{l}N L(\mathrm{I}) \\
\mathrm{g} / \mathrm{m}^{2}\end{array}$ & $\begin{array}{c}\mathrm{Li}, \\
\mathrm{mg} / \mathrm{L}\end{array}$ & $\begin{array}{c}N L(\mathrm{Li}) \\
\mathrm{g} / \mathrm{m}^{2}\end{array}$ & $\begin{array}{l}\mathrm{Na}, \\
\mathrm{mg} / \mathrm{L}\end{array}$ & $\begin{array}{c}N L(\mathrm{Na}) \\
\mathrm{g} / \mathrm{m}^{2}\end{array}$ & $\begin{array}{c}\mathrm{Si}, \\
\mathrm{mg} / \mathrm{L}\end{array}$ & $\begin{array}{c}N L(\mathrm{Si}) \\
\mathrm{g} / \mathrm{m}^{2}\end{array}$ \\
\hline RWS-NLS-7-a & 1 & .309 & 0.00099 & 0.298 & 0.00863 & 264 & 2.40 & 0.0229 & 0.0068 & 0.369 & 1.67 & 0.157 & 0.0155 & 131 & 0.492 & 4.76 & 0.0104 \\
\hline RWS-NLS-7-b & 1 & 321 & 0.00103 & & & 265 & 2.42 & 0.0235 & 0.0070 & 0.340 & 1.54 & 0.164 & 0.0162 & 128 & 0.481 & 4.68 & 0.0102 \\
\hline RWS-NLS-7-c & 1 & 0.301 & 0.00097 & & & 258 & 2.36 & 0.0292 & 0.0087 & 0.294 & 1.34 & 0.156 & 0.0155 & 125 & 0.472 & 4.42 & 0.0097 \\
\hline PCT-NLS-7-a & 1 & 18.0 & 0.0575 & 2.62 & 0.0758 & 59.3 & 0.540 & 0.207 & 0.0617 & 0.118 & 0.534 & 5.18 & 0.512 & 53.3 & 0.200 & 25.3 & 0.0552 \\
\hline PCT-NLS-7-b & 1 & 18.6 & 0.0599 & 2.68 & 0.0779 & 58.8 & 0.538 & 0.230 & 0.0688 & 0.114 & 0.518 & 5.27 & 0.523 & 55.6 & 0.209 & 25.8 & 0.0567 \\
\hline PCT-NLS-7-c & 1 & 16.7 & 0.0534 & 2.60 & 0.0755 & 59.0 & 0.538 & 0.213 & 0.0634 & 0.112 & 0.507 & 5.04 & 0.498 & 49.7 & 0.186 & 23.5 & 0.0513 \\
\hline RWS-NLS-9R-a & 4 & 0.199 & 0.00064 & 0.257 & 0.00757 & 432 & 3.99 & 0.0386 & 0.0117 & 1.02 & 4.69 & 0.395 & 0.0397 & 274 & 1.04 & 8.36 & 0.0185 \\
\hline RWS-NLS-9R-b & 4 & 142 & 0.00046 & & & 474 & 4.40 & 0.0420 & 0.0127 & 0.997 & 4.60 & 0.412 & 0.0415 & 321 & 1.23 & 8.21 & 0.0183 \\
\hline RWS-NLS-9R-C & 4 & 279 & 0.00089 & & & 478 & 4.36 & 0.0412 & 0.0123 & 0.856 & 3.88 & 0.392 & 0.0387 & 305 & 1.14 & 7.83 & 0.0171 \\
\hline PCT-NLS-9R-a & $\overline{4}$ & 8.8 & 0.0606 & 1.85 & 0.0540 & 43.9 & 0.403 & 0.175 & 0.0525 & 0.082 & 0.373 & 5.27 & 0.525 & 33.5 & 0.127 & 27.7 & 0.0610 \\
\hline PCT-NLS-9R-b & 4 & & 610 & 1.83 & 0530 & 43.3 & 0.394 & 0.165 & 0.0491 & 0.079 & 0.358 & 5.37 & .530 & 35.6 & 0.133 & 28.1 & 0.0613 \\
\hline PCT-NLS-9R-C & 4 & 18.7 & 0.0599 & 1.82 & 0.0528 & 42.0 & 0.383 & 0.169 & 0.0504 & 0.078 & 0.355 & 5.31 & 0.525 & 34.5 & 0.129 & 27.6 & 0.0604 \\
\hline RWS-NLS-25-a & 8 & 0.346 & 0.00111 & 0.231 & 0.00671 & 530 & 4.84 & 0.0490 & 0.0146 & 1.06 & 4.81 & 0.492 & 0.0487 & 303 & 1.14 & 6.53 & 0.0143 \\
\hline RWS-NLS-25-b & 8 & 272 & 0.00091 & & & 537 & 5.15 & 0.0492 & 0.0154 & 0.965 & 4.60 & 0.487 & 0.0506 & 312 & 1.23 & 6.92 & 0.0159 \\
\hline RWS-NLS-25-c & 8 & 0.243 & 0.00078 & & & 533 & 4.87 & 0.0474 & 0.0142 & 0.931 & 4.23 & 0.486 & 0.0482 & 297 & 1.12 & 7.28 & 0.0160 \\
\hline PCT-NLS-25-a & 8 & 20.1 & 0.0641 & 1.23 & 355 & 39.4 & 0.359 & 0.188 & 0.0561 & 0.088 & 0.399 & 5.23 & 0.516 & 36.4 & 0.136 & 32.2 & 0.0704 \\
\hline $25-b$ & 8 & 4 & 653 & 1.24 & 59 & 40.1 & 0.365 & 0.186 & 0.0554 & 0.086 & 0.388 & 5.30 & 0.524 & 36.8 & 138 & 32.9 & 0.0719 \\
\hline $25-c$ & 8 & 0.4 & 0.0650 & 1.21 & 0.0350 & 41.2 & 0.375 & 0.182 & 0.0543 & 0.087 & 0.395 & 5.33 & 0.526 & 36.9 & 0.138 & 32.4 & 0.0706 \\
\hline S-31-a & 16 & 372 & 0.00121 & 0.199 & 0.00585 & 469 & 4.33 & 0.0387 & 0.0117 & 0.868 & 3.99 & 0.403 & 0.0404 & 256 & 0.972 & 6.24 & 0.0138 \\
\hline S-31-b & 1 & 337 & 0.00109 & & & 511 & 4.71 & 0.0403 & .0121 & 0.991 & 4.54 & 0.419 & 0.0419 & 274 & 1.04 & 7.51 & 0.0166 \\
\hline$-31-c$ & $T$ & 0.333 & 0.00109 & & & 511 & 4.77 & 0.0407 & .0124 & 1.52 & 7.05 & 0.415 & 0.0420 & 291 & 1.12 & 7.48 & 0.0167 \\
\hline PCT-NLS-31-a & 16 & 9.2 & 0.0613 & 1.48 & 0.0430 & 43.2 & 0.394 & 0.132 & 0.0394 & 0.088 & 0.397 & 5.01 & 0.496 & 39.2 & 0.147 & 31.0 & 0.0677 \\
\hline PCT-NLS-31-b & 16 & 19.3 & 0.0616 & 1.61 & 0.0465 & 43.4 & 0.395 & 0.139 & 0.0415 & 0.088 & 0.399 & 4.73 & 0.468 & 39.6 & 0.148 & 31.1 & 0.0679 \\
\hline $31-c$ & 16 & 19.3 & 0615 & 1.68 & 0.0488 & 43.2 & 0.394 & 0.151 & 0.0450 & 0.087 & 0.392 & 5.00 & 0.494 & 40.3 & 0.151 & 31.7 & 0.0691 \\
\hline RWS-NLS-32-a & 24 & 306 & 0.00098 & 0.195 & 0.00 & 601 & 5.48 & 0.0497 & & 2.13 & 9.65 & 0.436 & 0.0431 & 308 & 1.16 & 6.88 & 0.0150 \\
\hline $32-b$ & 20 & 241 & 0.00076 & & & 613 & 5.52 & 66 & & 1.45 & 6.48 & 0.431 & 20 & 332 & 1.23 & 6.70 & 0.0144 \\
\hline RWS-I & 24 & 324 & 0.00106 & & & 626 & 5.83 & 0.0456 & 0.0139 & 1.26 & 5.83 & 0.462 & 0.0466 & 327 & 1.25 & 7.77 & 0.0173 \\
\hline PCT-NLS-32-a & 24 & 8.7 & 0.0572 & 61 & 474 & 46.2 & 0.427 & 53 & 0.0463 & 0.104 & 0.477 & 4.94 & 0.495 & 41.3 & 0.157 & 30.3 & 0.0670 \\
\hline $32-b$ & 24 & .8 & 0.06021 & 1.62 & 0470 & 46.2 & 0.421 & 0.149 & 0.0443 & 0.104 & 0.471 & 4.93 & 0.487 & 42.2 & 0.158 & 30.3 & 0.0662 \\
\hline PCT-NLS-32-c & 24 & 18.3 & 0.0583 & 1.57 & 0.0456 & 46.5 & 0.423 & 0.148 & 0.0441 & 0.113 & 0.511 & 5.15 & 0.508 & 44.2 & 0.165 & 30.6 & 0.0667 \\
\hline RWS-NLS-6-a & 36 & 0.201 & 0.00064 & 0.210 & 0.00611 & 438 & 4.01 & 0.0258 & 0.0077 & 1.11 & 5.04 & 0.187 & 0.0185 & 243 & 0.915 & 6.35 & 0.0139 \\
\hline RWS-NLS-6-b & 36 & 0.222 & 0.00071 & & & 430 & 3.83 & 0.0260 & 0.0077 & 1.11 & 5.03 & 0.189 & 0.0187 & 250 & 0.936 & 5.36 & 0.0117 \\
\hline RWS-NLS-6-c & 36 & 0.219 & 0.00070 & & & 457 & 4.17 & 0.0269 & 0.0080 & 1.12 & 5.07 & 0.208 & 0.0206 & 256 & 0.958 & 5.40 & 0.0118 \\
\hline PCT-NLS-6-a & 36 & 18.5 & 0.0590 & 2.25 & 0.0651 & 61.1 & 0.556 & 0.214 & 0.0636 & 0.128 & 0.579 & 5.51 & 0.544 & 50.1 & 0.187 & 27.3 & 0.0594 \\
\hline PCT-NLS-6-b & 36 & 18.4 & 0.0590 & 2.13 & 0.0620 & 63.7 & 0.582 & 0.210 & 0.0628 & 0.122 & 0.554 & 5.19 & 0.514 & 53.1 & 0.200 & 27.5 & 0.0603 \\
\hline PCT-NLS-6-C & 36 & 15.5 & 0.0496 & 2.14 & 0.0619 & 64.0 & 0.583 & 0.210 & 0.0624 & 0.125 & 0.566 & 4.68 & 0.462 & 45.5 & 0.170 & 22.9 & 0.0500 \\
\hline
\end{tabular}


Table 46. Results of PCT with T-t PC CWF Products, $N L(i)$ in $\mathrm{g} / \mathrm{m}^{2}$

\begin{tabular}{|c|c|c|c|c|c|c|c|c|c|}
\hline Test No. & Time, $\mathrm{h}$ & $N L(\mathrm{Al})$ & $N L(\mathrm{~B})$ & $N L(\mathrm{Cl})$ & $N L(\mathrm{Cs})$ & $N L(\mathrm{I})$ & $N L(\mathrm{Li})$ & $N L(\mathrm{Na})$ & $N L(\mathrm{Si})$ \\
\hline \multicolumn{10}{|c|}{ Processing Temperature $=850^{\circ} \mathrm{C}$} \\
\hline NLS-2 & 1 & 0.114 & 1.43 & 0.306 & 0.125 & 0.253 & 0.887 & 0.239 & 0.109 \\
\hline NLS-15 & 4 & 0.103 & 0.145 & 0.639 & 0.0606 & 0.676 & 0.608 & 0.370 & 0.0940 \\
\hline NLS-17 & 8 & 0.0847 & 0.0657 & 1.23 & 0.0428 & 1.68 & 0.551 & 0.490 & 0.0857 \\
\hline NLS-26 & 16 & 0.0896 & 0.0353 & 1.63 & 0.0341 & 1.79 & 0.487 & 0.517 & 0.0930 \\
\hline NLS-24 & 24 & 0.0832 & 0.0296 & 1.81 & 0.0349 & 2.24 & 0.490 & 0.557 & 0.0912 \\
\hline NLS-27 & 36 & 0.0829 & 0.0317 & 1.77 & 0.0318 & 1.94 & 0.511 & 0.544 & 0.0911 \\
\hline \multicolumn{10}{|c|}{ Processing Temperature $=875^{\circ} \mathrm{C}$} \\
\hline NLS-3 & 1 & 0.108 & 0.467 & 0.339 & 0.0630 & 0.378 & 0.551 & 0.212 & 0.0912 \\
\hline NLS-14 & 4 & 0.0863 & 0.0958 & 1.33 & 0.0478 & 1.27 & & 0.474 & 0.0872 \\
\hline NLS-19 & 8 & 0.0715 & 0.0409 & 2.12 & 0.0445 & 1.90 & 0.622 & 0.612 & 0.0808 \\
\hline NLS-28 & 16 & 0.0629 & 0.0330 & 2.33 & 0.0581 & 2.80 & 0.479 & 0.626 & 0.0789 \\
\hline NLS-33 & 24 & 0.0664 & 0.0326 & 2.31 & 0.0392 & 2.76 & 0.419 & 0.639 & 0.0847 \\
\hline NLS-23 & 36 & 0.0837 & 0.0208 & 1.92 & 0.0328 & 2.37 & 0.473 & 0.593 & 0.0937 \\
\hline \multicolumn{10}{|c|}{ Processing Temperature $=900^{\circ} \mathrm{C}$} \\
\hline NLS-4 & 1 & 0.0980 & 0.184 & 0.769 & 0.0553 & 0.793 & 0.476 & 0.281 & 0.0898 \\
\hline NLS-10 & 4 & 0.0751 & 0.0510 & 2.55 & 0.0551 & 5.02 & 0.621 & 0.748 & 0.0853 \\
\hline NLS-20 & 8 & 0.0623 & 0.0463 & 3.31 & 0.0607 & 2.87 & 0.666 & 0.791 & 0.0824 \\
\hline NLS-29 & 16 & 0.0822 & 0.0348 & 2.84 & 0.0493 & 2.96 & 0.546 & 0.760 & 0.0991 \\
\hline NLS-34 & 24 & 0.0715 & 0.0308 & 2.86 & 0.0426 & 3.43 & 0.432 & 0.798 & 0.0847 \\
\hline NLS-18 & 36 & 0.0715 & 0.0445 & 2.71 & 0.0494 & 2.44 & 0.621 & 0.681 & 0.0873 \\
\hline \multicolumn{10}{|c|}{ Processing Temperature $=915^{\circ} \mathrm{C}$} \\
\hline NLS-1 & 1 & 0.0875 & 0.132 & 1.28 & 0.0572 & 1.38 & 0.463 & 0.366 & 0.0790 \\
\hline NLS-13 & 4 & 0.0638 & 0.0828 & 3.17 & 0.0643 & 2.97 & 0.549 & 0.867 & 0.0795 \\
\hline NLS-21 & 8 & 0.0663 & 0.0431 & 3.21 & 0.0583 & 3.10 & 0.675 & 0.876 & 0.0793 \\
\hline NLS-8 & 16 & 0.0797 & 0.0640 & 2.96 & 0.0440 & 4.15 & 0.498 & 0.747 & 0.0855 \\
\hline NLS-36 & 24 & 0.0733 & 0.0332 & 3.15 & 0.0404 & 3.67 & 0.452 & 0.862 & 0.0884 \\
\hline NLS-16 & 36 & 0.0636 & 0.0492 & 3.85 & 0.0819 & 4.36 & 0.598 & 0.990 & 0.0827 \\
\hline \multicolumn{10}{|c|}{ Processing Temperature $=925^{\circ} \mathrm{C}$} \\
\hline NLS-5 & 1 & 0.0810 & 0.106 & 1.54 & 0.0464 & 1.52 & 0.459 & 0.424 & 0.0791 \\
\hline NLS-12 & 4 & 0.0513 & 0.0859 & 3.43 & 0.0760 & 3.28 & 0.566 & 0.925 & 0.0668 \\
\hline NLS-22 & 8 & 0.0633 & 0.0327 & 3.70 & 0.0613 & 4.05 & 0.550 & 0.986 & 0.0840 \\
\hline NLS-30 & 16 & 0.0659 & 0.0454 & 3.50 & 0.0510 & 3.64 & 0.454 & 0.877 & 0.0890 \\
\hline NLS-35 & 24 & 0.0720 & 0.0338 & 4.18 & 0.0488 & 4.14 & 0.458 & 1.06 & 0.0892 \\
\hline NLS-11 & 36 & 0.0552 & 0.0534 & 4.14 & 0.0684 & 3.81 & 0.573 & 1.042 & 0.0728 \\
\hline \multicolumn{10}{|c|}{ Processing Temperature $=950^{\circ} \mathrm{C}$} \\
\hline NLS-7 & 1 & 0.0579 & 0.0764 & 2.93 & 0.0721 & 2.04 & 0.526 & 0.680 & 0.0645 \\
\hline NLS-9R & 4 & 0.0611 & 0.0533 & 4.64 & 0.0629 & 4.75 & 0.567 & 1.267 & 0.0789 \\
\hline NLS-25 & 8 & 0.0657 & 0.0355 & 5.32 & 0.0700 & 4.94 & 0.571 & 1.230 & 0.0863 \\
\hline NLS-31 & 16 & 0.0626 & 0.0461 & 5.00 & 0.0540 & 5.59 & 0.527 & 1.19 & 0.0840 \\
\hline NLS-32 & 24 & 0.0595 & 0.0467 & 6.03 & 0.0590 & 7.80 & 0.541 & 1.37 & 0.0822 \\
\hline NLS-6 & 36 & 0.0566 & 0.0630 & 4.57 & 0.0708 & 5.62 & 0.526 & 1.12 & 0.0691 \\
\hline
\end{tabular}




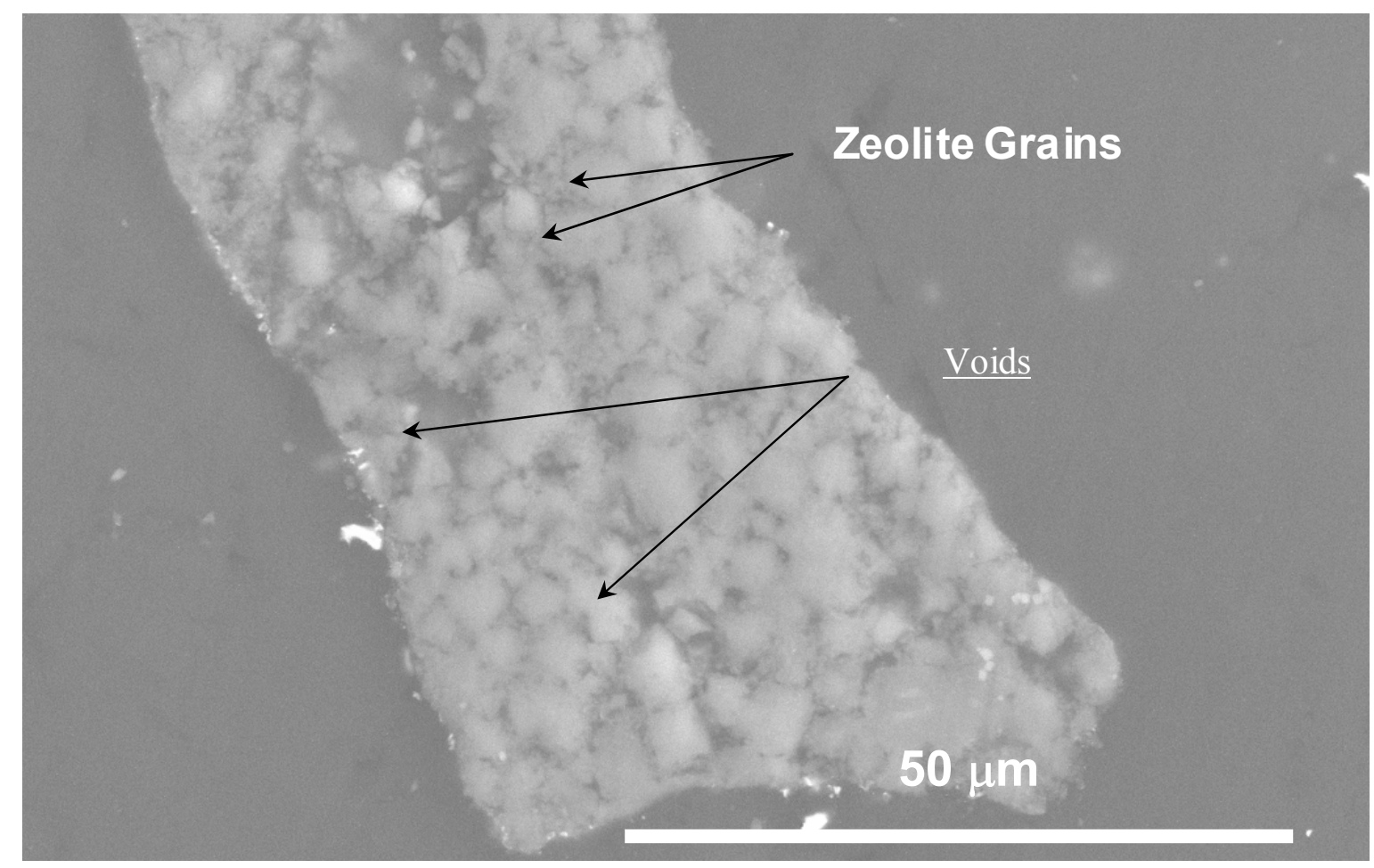

(a)

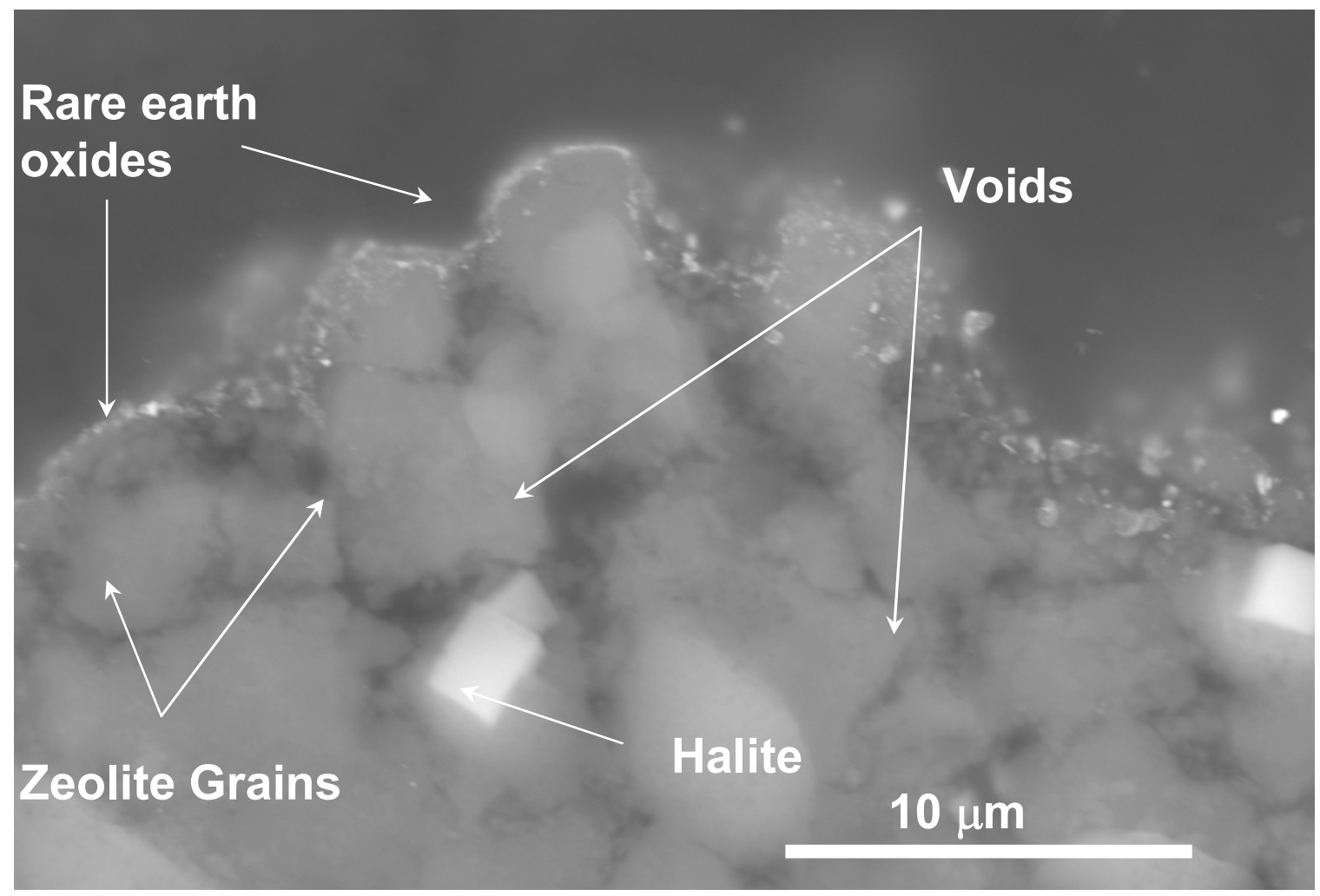

(b)

Fig. 1. SEM Image of Salt-Loaded Zeolite at (a) Low Magnification and (b) High Magnification. 


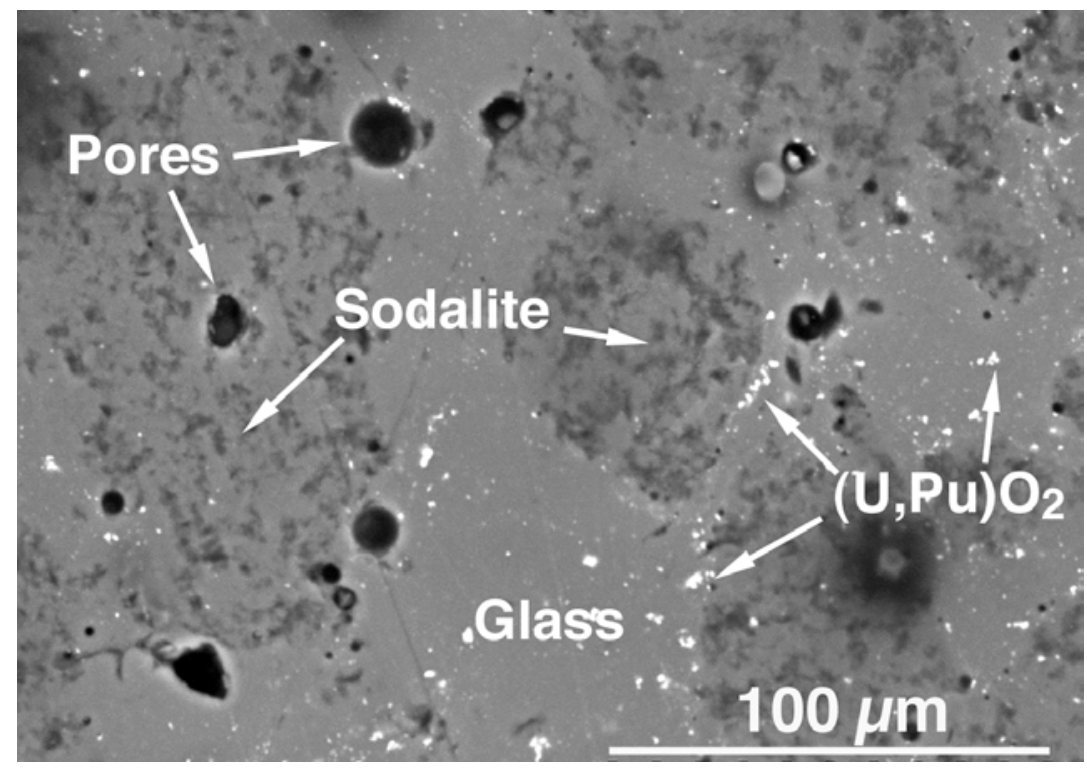

(a)

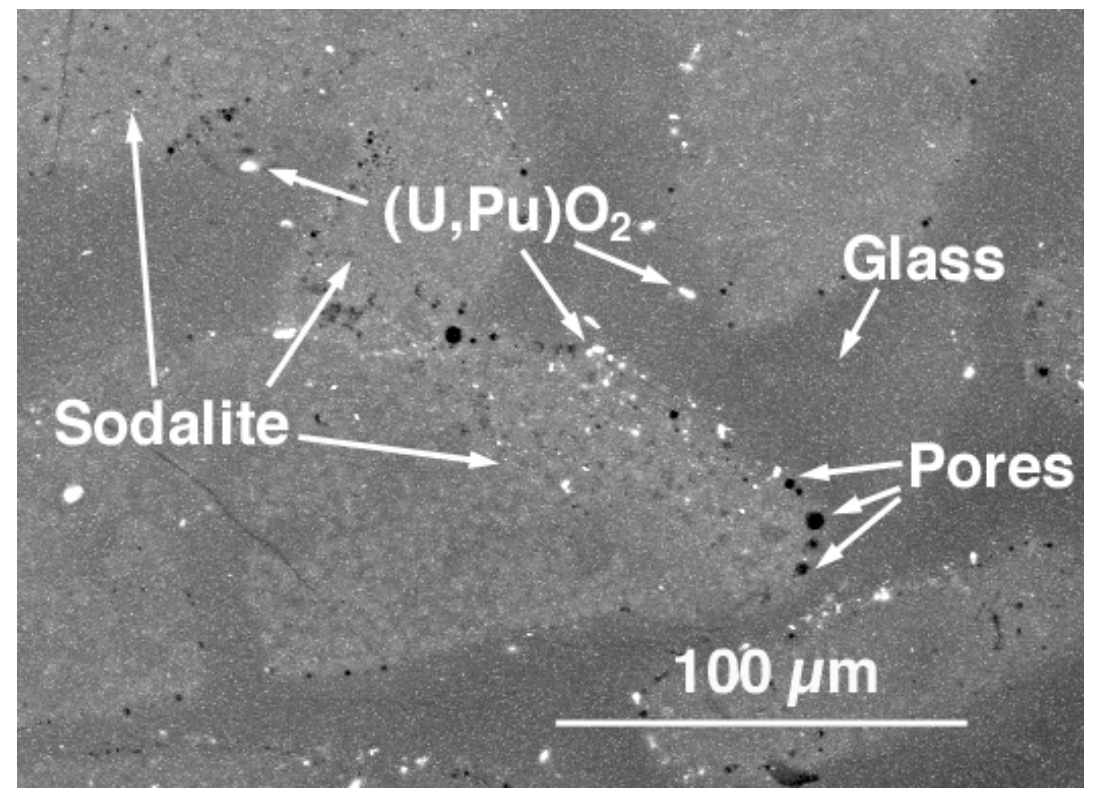

(b)

Fig. 2. Photomicrographs Showing Microstructures of (a) U,Pu-Doped PC CWF and (b) U,Pu-Doped HIP CWF. 


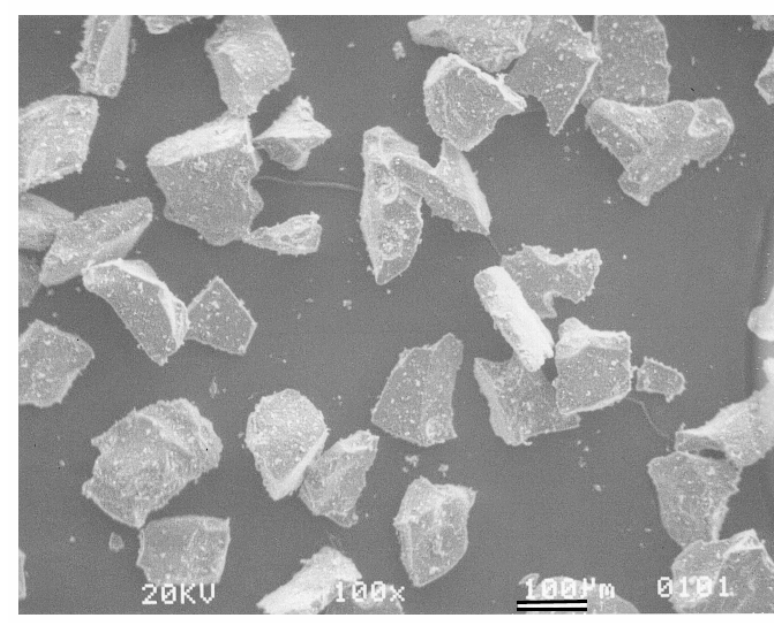

(a)

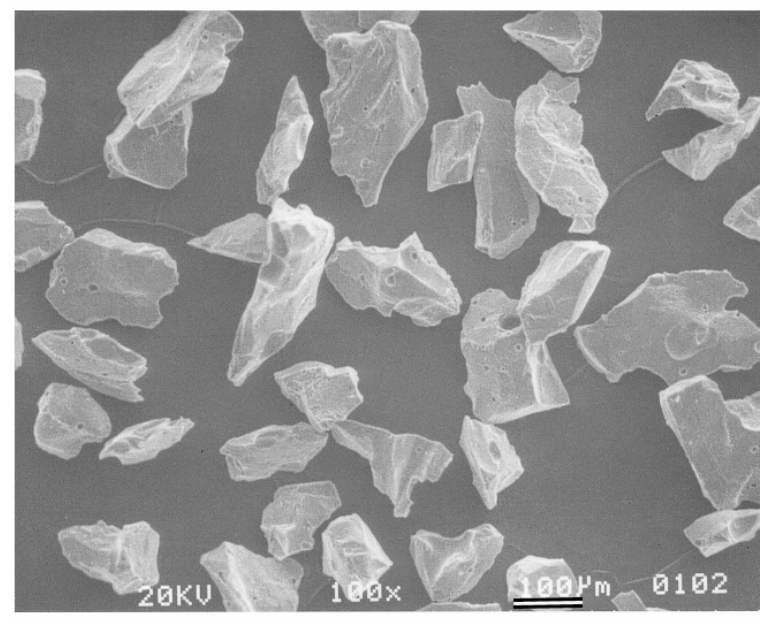

(c)

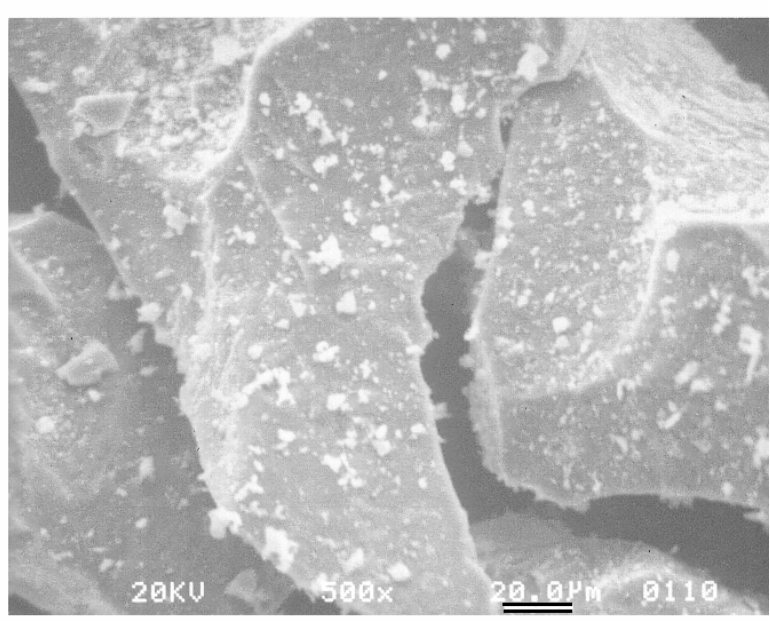

(b)

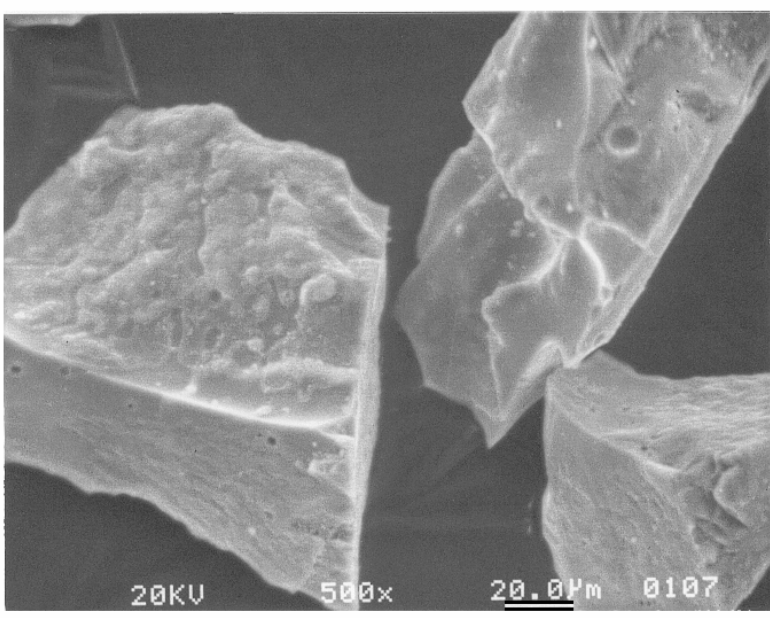

(d)

Fig. 3. Scanning Electron Microscopy Photomicrographs of $-100+200$ Mesh Size Fraction PC CWF before ( $a$ and b) and after ( $c$ and d) Subjection to the Washing Procedure. 


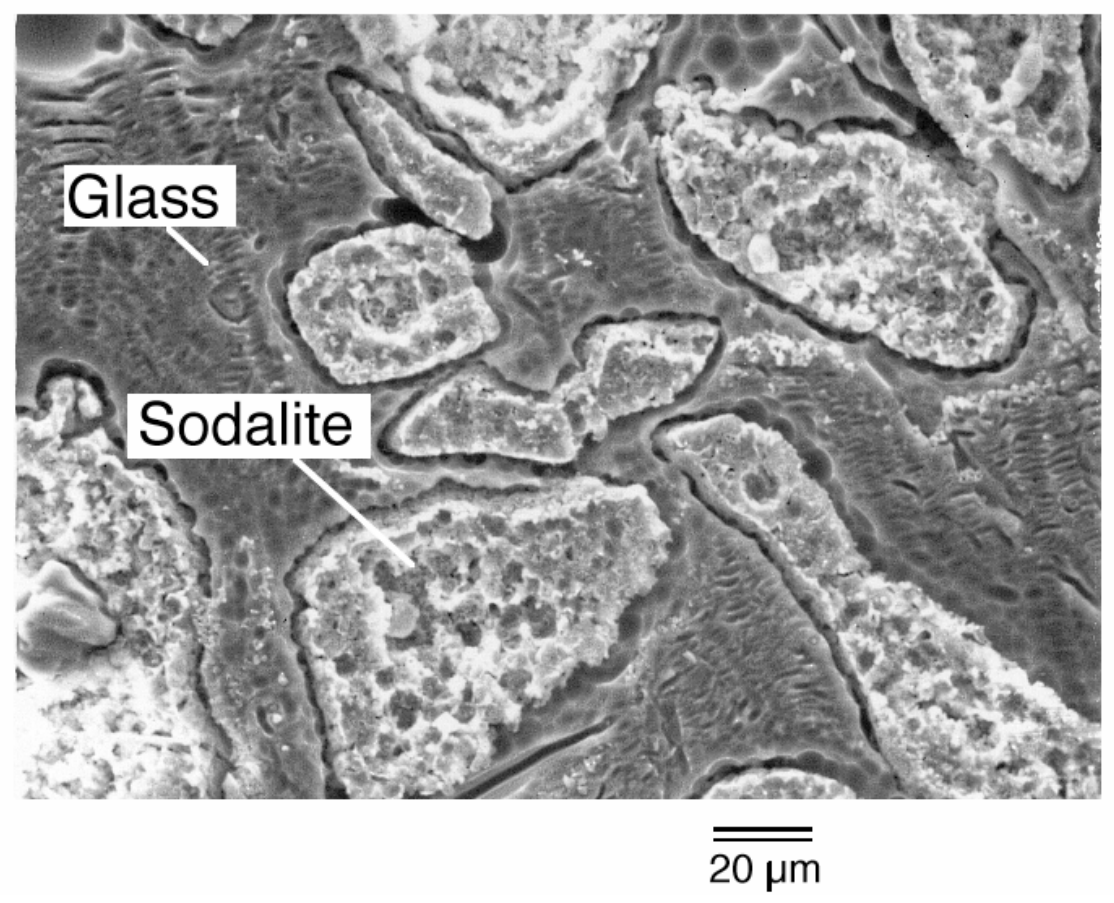

(a)

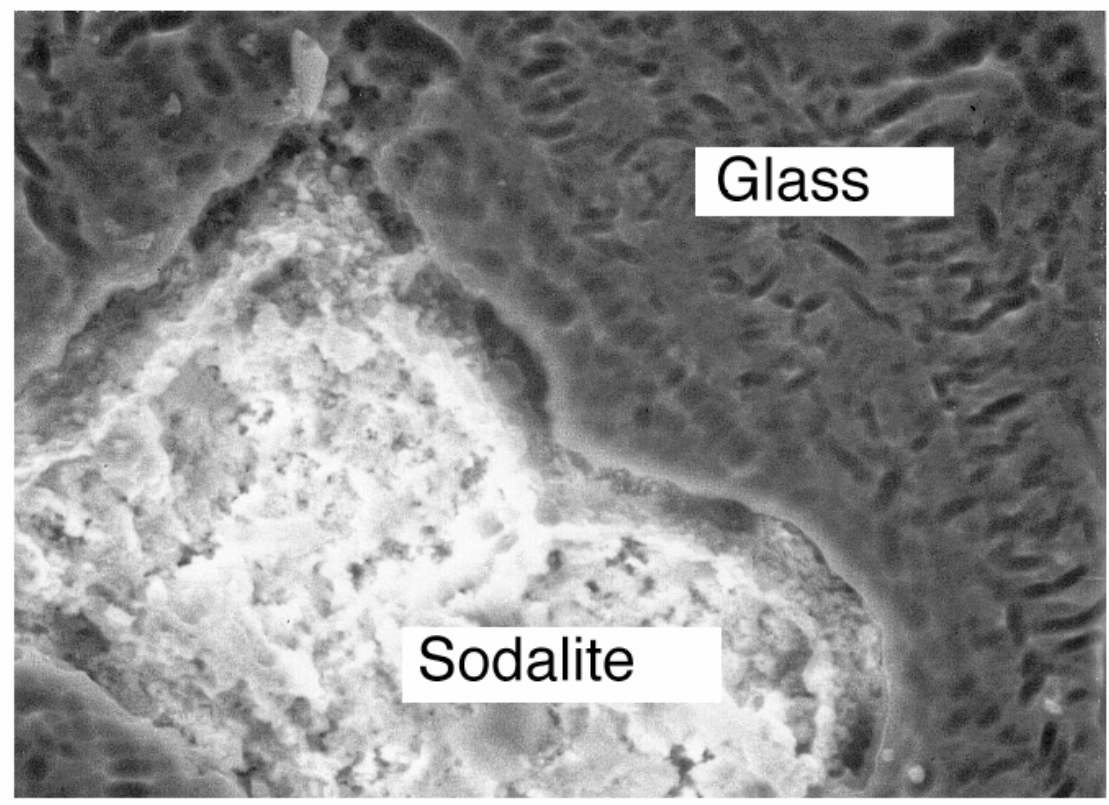

(b)

Fig. 4. Photomicrographs Showing Surfaces of (a) Baseline PC CWF and (b) HIP CWF after 88-Day MCC-1 Test at $125^{\circ} \mathrm{C}$. 


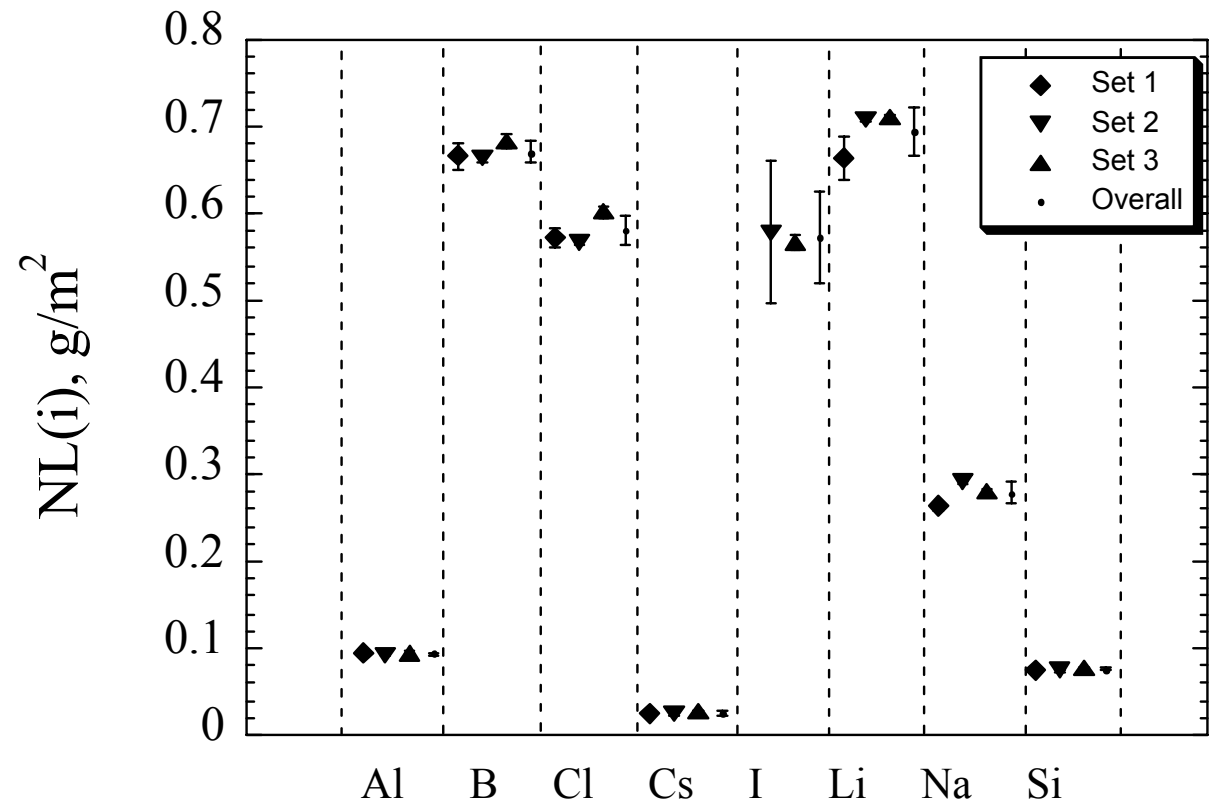

Fig. 5. Mean Total $N L(i)$ for Three Sets of Triplicate 7-Day PCTs with the 5-kg Baseline PC CWF. Uncertainty bars drawn for one standard deviation. 


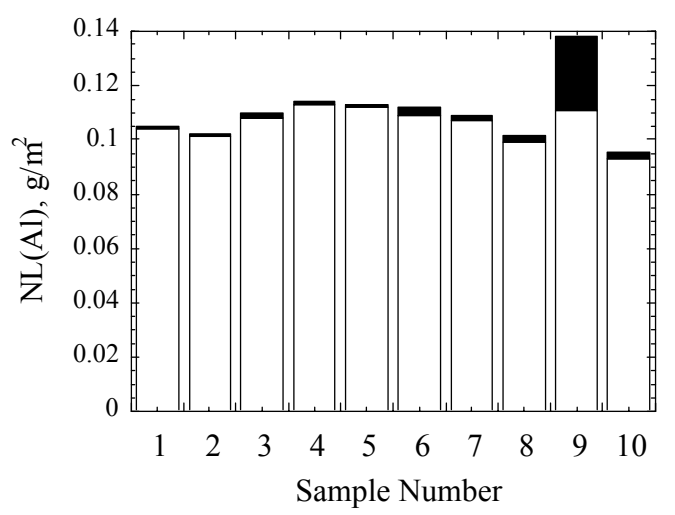

(a)

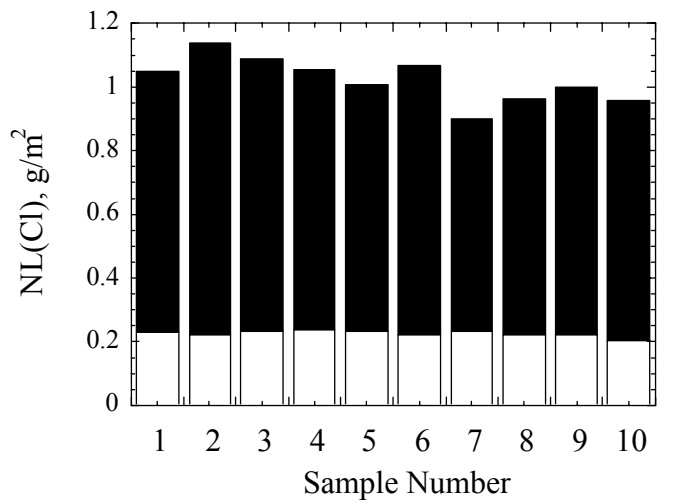

(c)

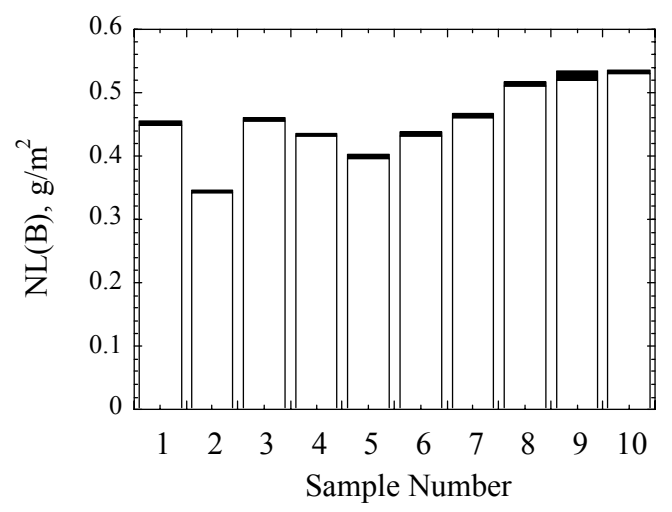

(b)

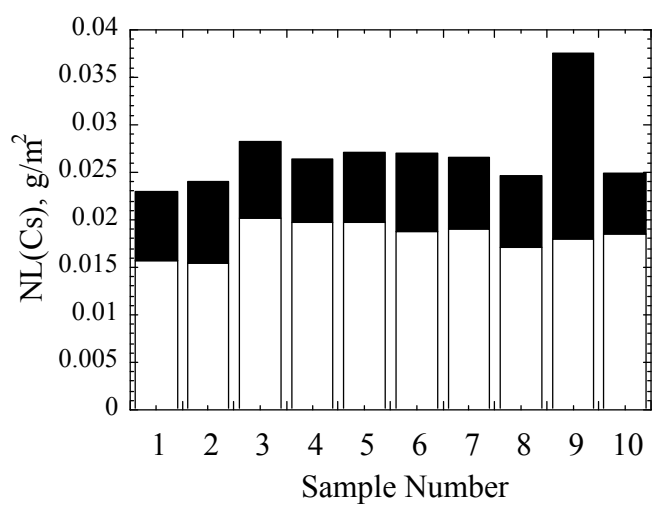

(d)

Fig. 6. Contributions to $N L(i)$ of RWS Solution (filled) and PCT Solution (open) in 7Day PCTs with Replicate DTD Baseline PC CWF for (a) $N L(\mathrm{Al})$, (b) $N L(\mathrm{~B})$, (c) $N L(\mathrm{Cl}),(\mathrm{d}) N L(\mathrm{Cs}),(\mathrm{e}) N L(\mathrm{I}),(\mathrm{f}) N L(\mathrm{Li}),(\mathrm{g}) N L(\mathrm{Na})$, and (h) $N L(\mathrm{Si})$. 


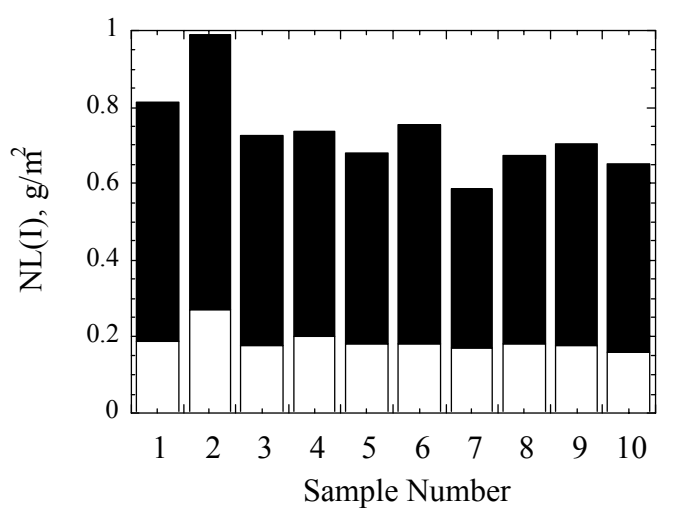

(e)

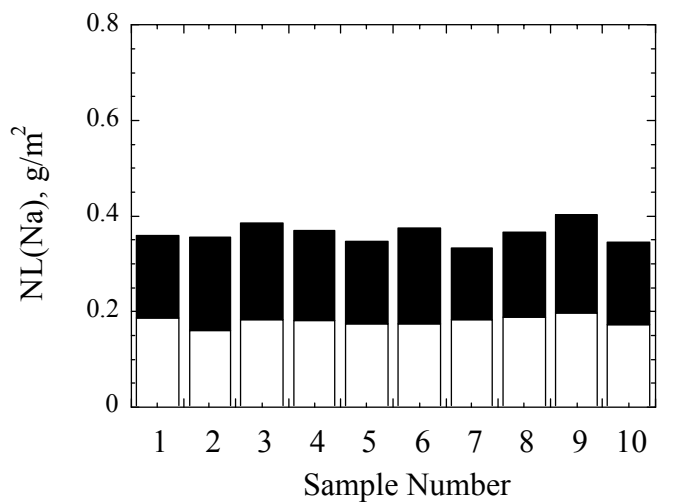

(g)

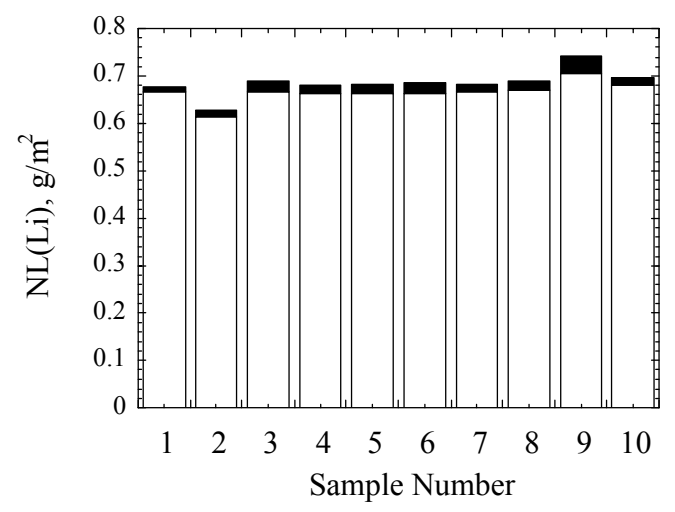

(f)

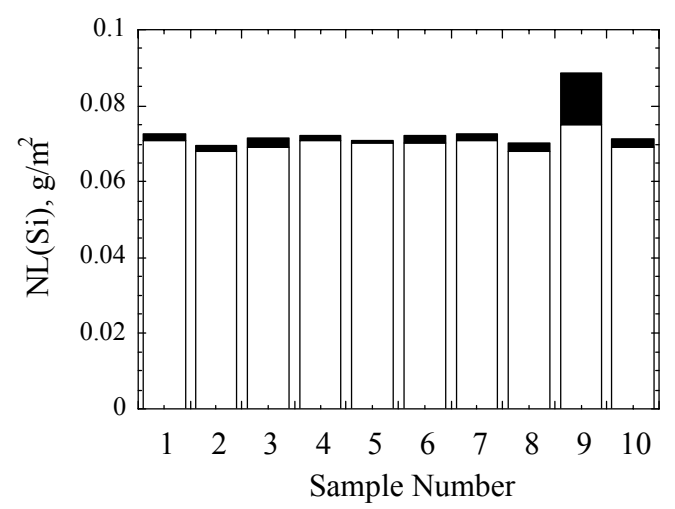

(h)

Fig. 6. (Contd.) 


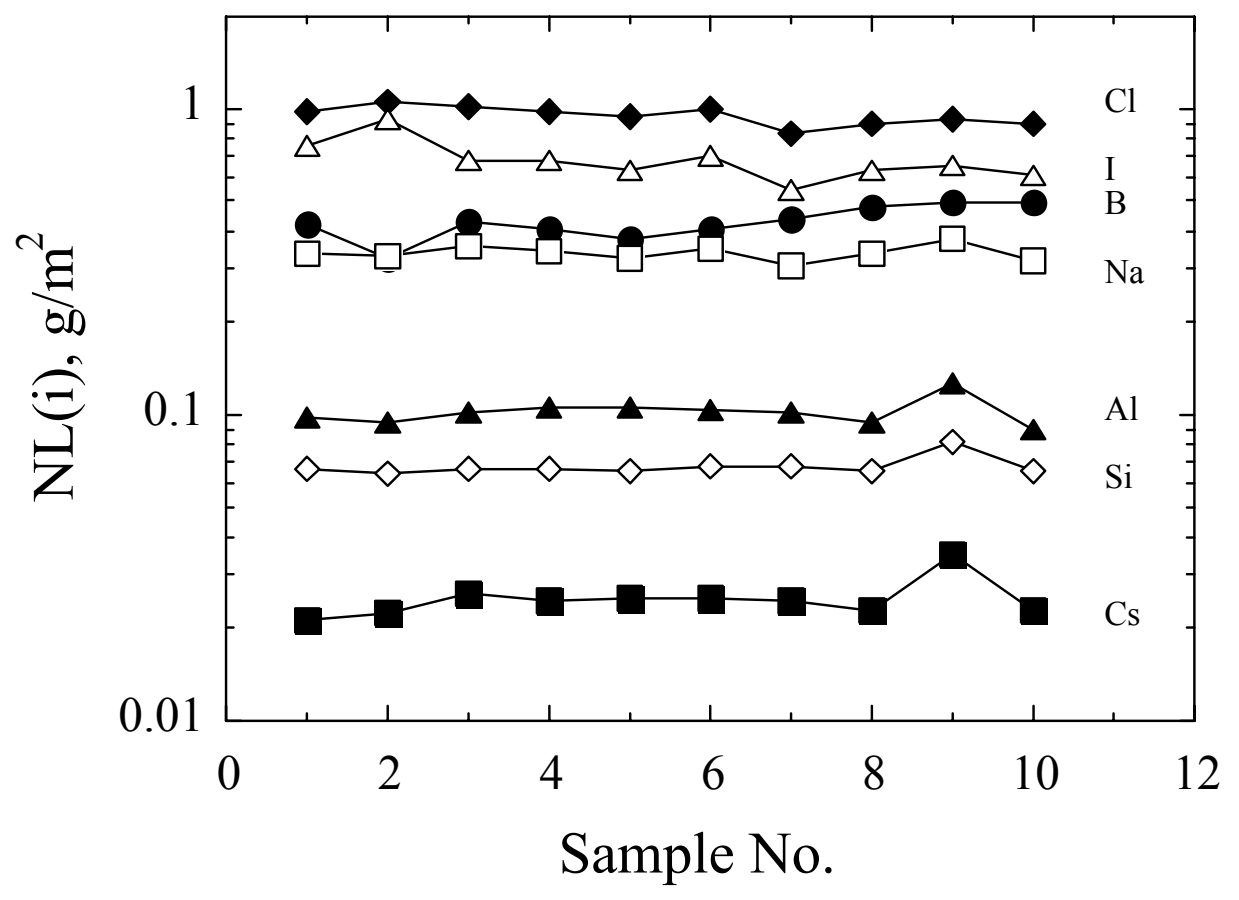

Fig. 7. Summary Plot of Total $N L(i)$ for Replicate DTD Baseline PC CWF Samples. 


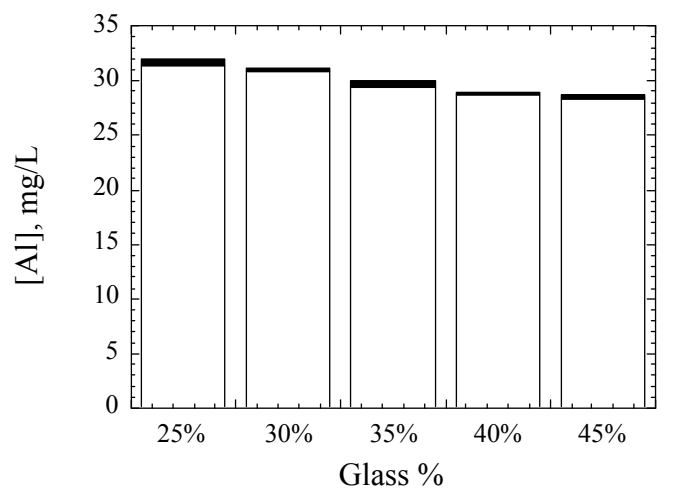

(a)

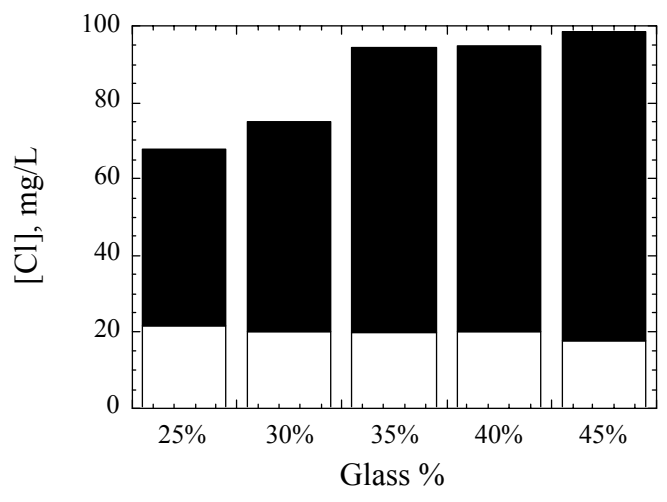

(c)

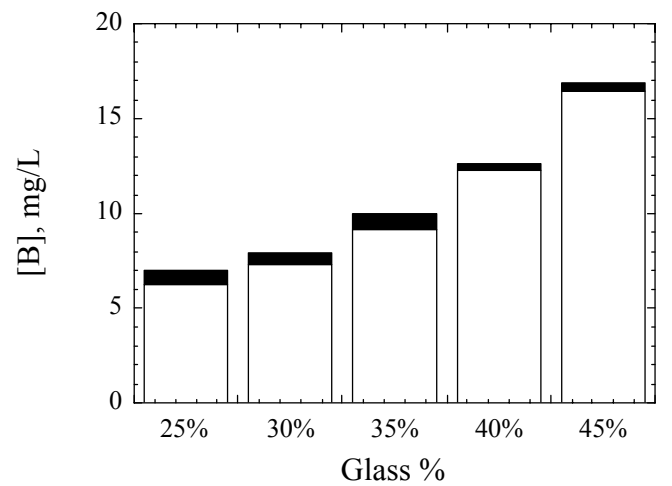

(b)

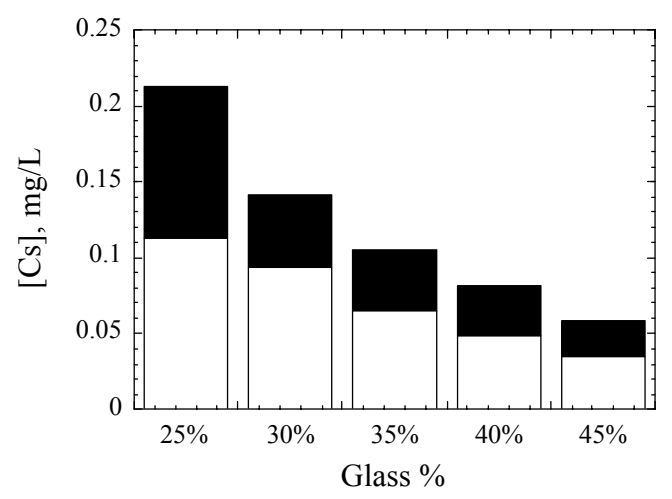

(d)

Fig. 8. Contributions to Concentration from RWS Solution (filled) and PCT Solution (open) in Triplicate 7-Day PCTs with Glass Loading PC CWF for (a) Al, (b) B, (c) Cl, (d) Cs, (e) I, (f) Li, (g) Na, and (h) Si. 


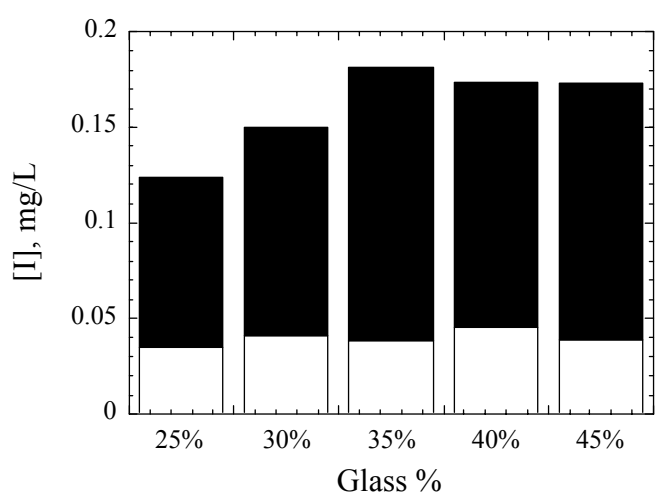

(e)

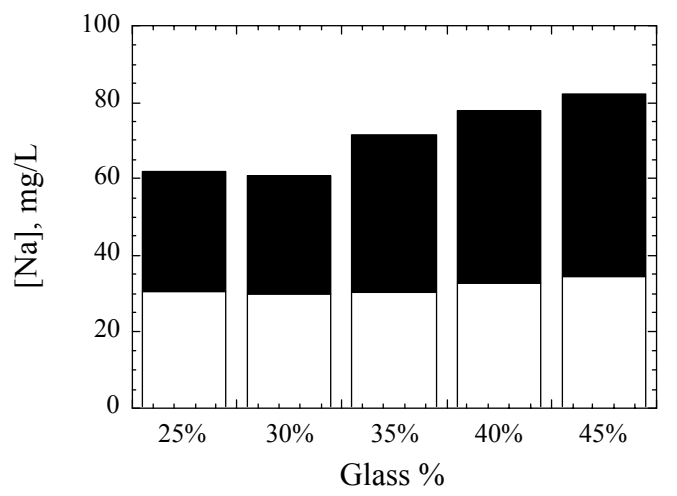

(g)

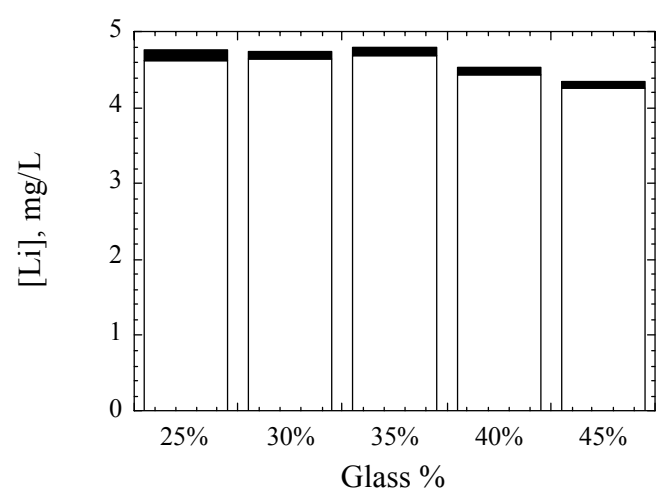

(f)

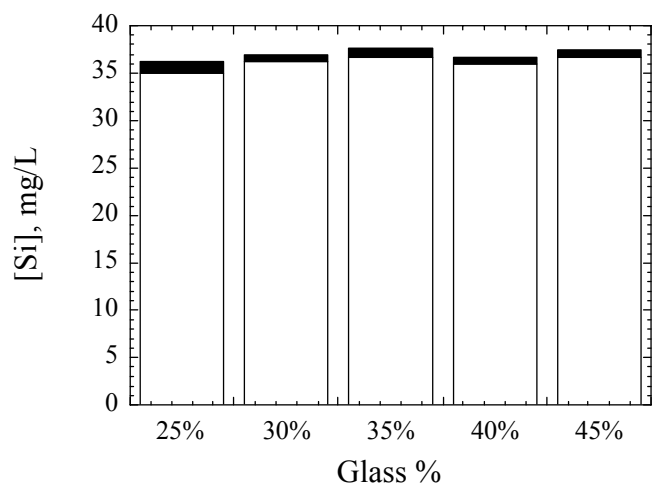

(h)

Fig. 8. (Contd.) 


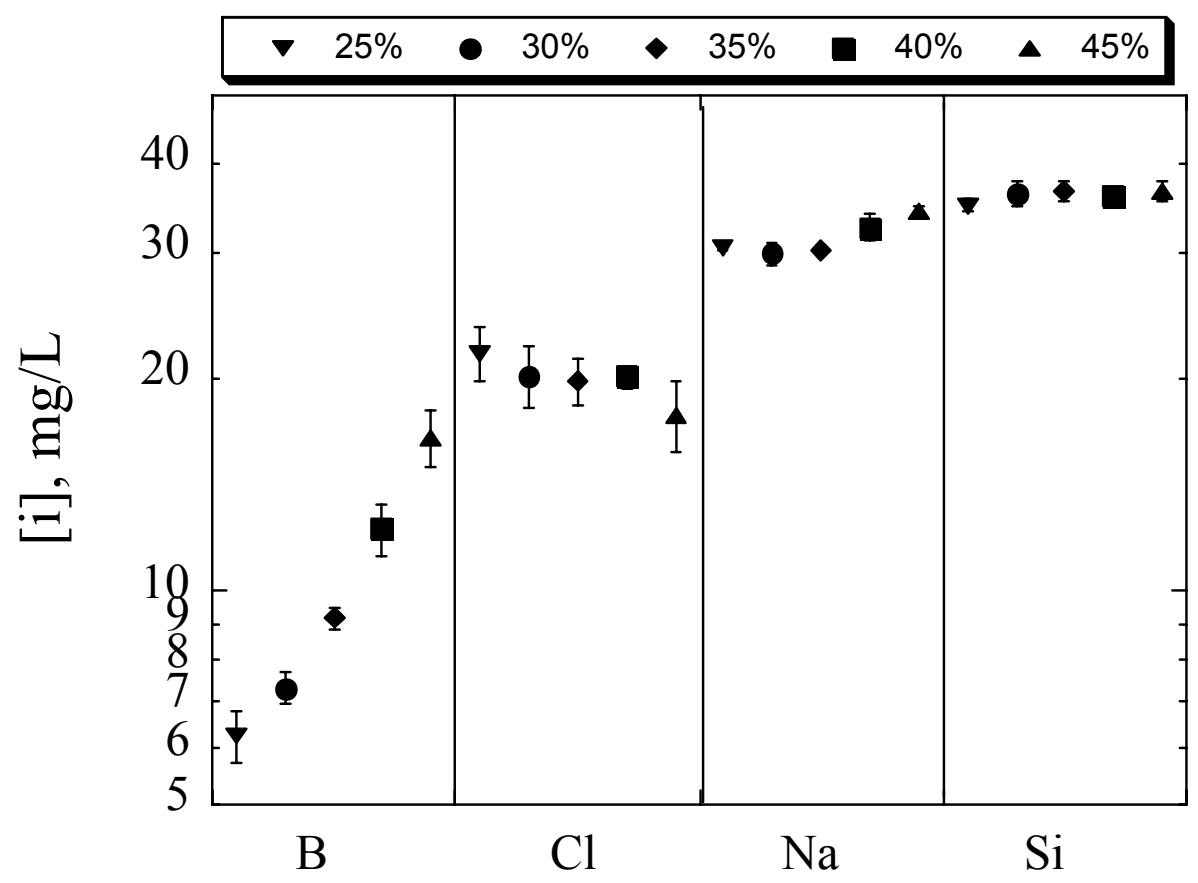

Fig. 9. Mean Concentrations ( \pm Two Standard Deviations) in PCT Fraction of 7-Day PCTs with Glass Loading PC CWF. 


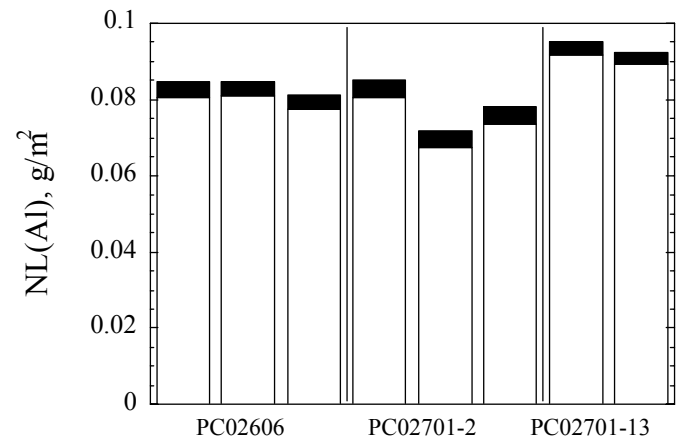

(a)

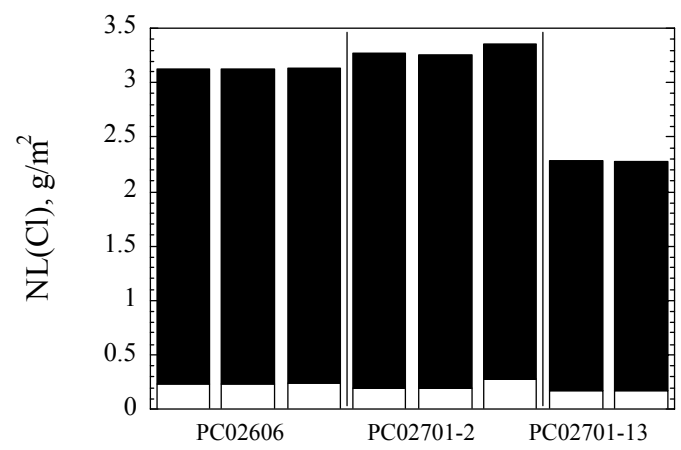

(c)

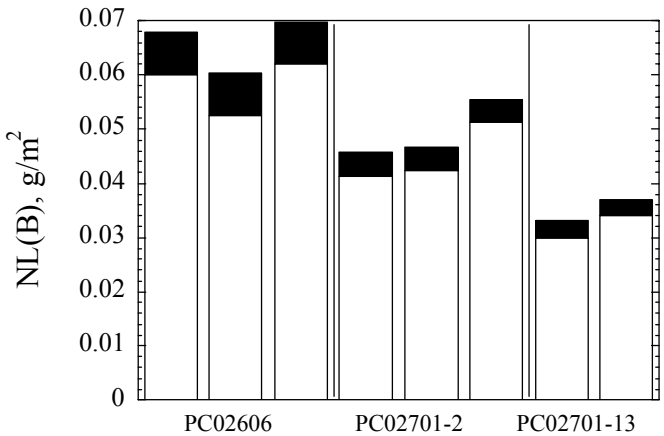

(b)

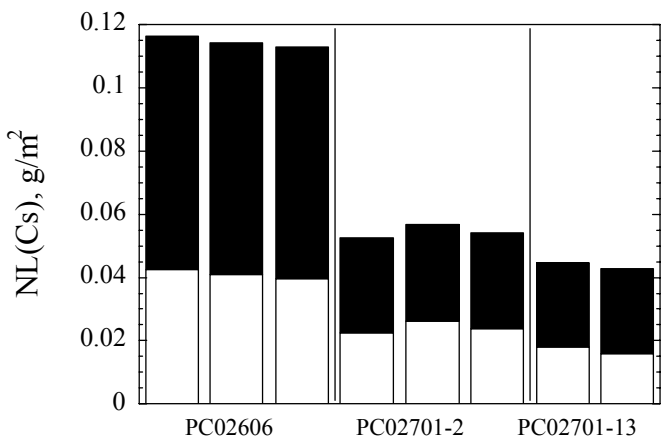

(d)

Fig. 10. Contribution to $N L(i)$ of RWS Solution (filled) and PCT Solution (open) in 7-Day PCTs with Advanced PC CWF for (a) $N L(\mathrm{Al})$, (b) $N L(\mathrm{~B})$, (c) $N L(\mathrm{Cl})$, (d) $N L(\mathrm{Cs})$, (e) $N L(\mathrm{I}),(\mathrm{f}) N L(\mathrm{Li}),(\mathrm{g}) N L(\mathrm{Na})$, and (h) $N L(\mathrm{Si})$. 


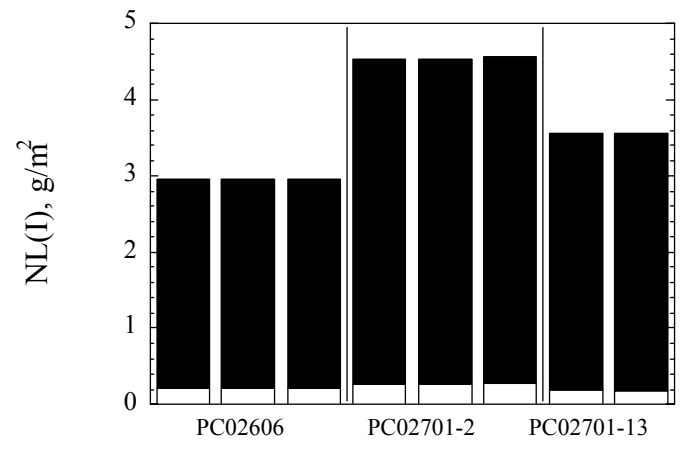

(e)

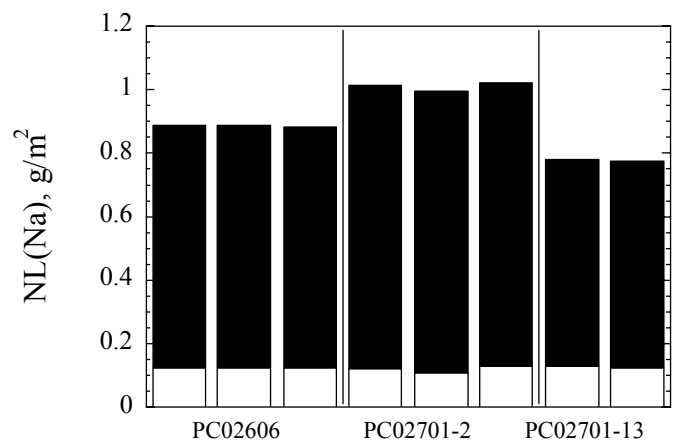

(g)

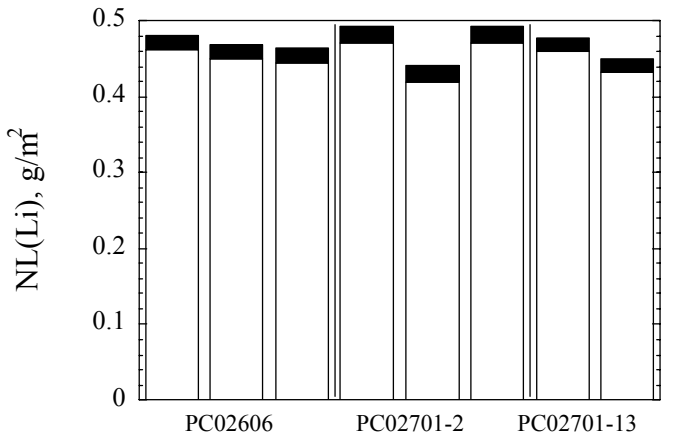

(f)

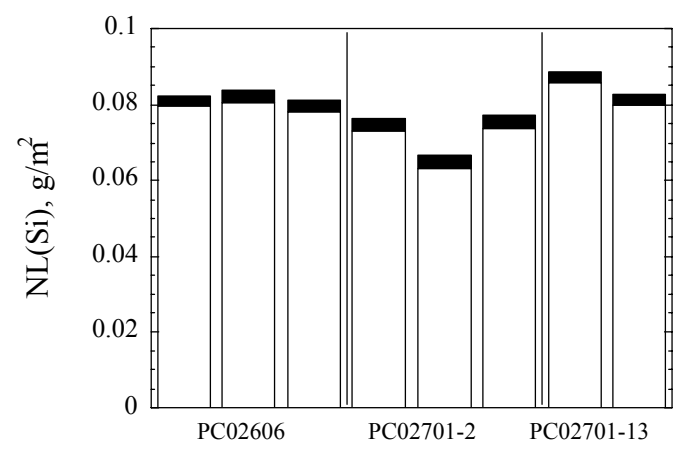

(h)

Fig. 10. (Contd.) 


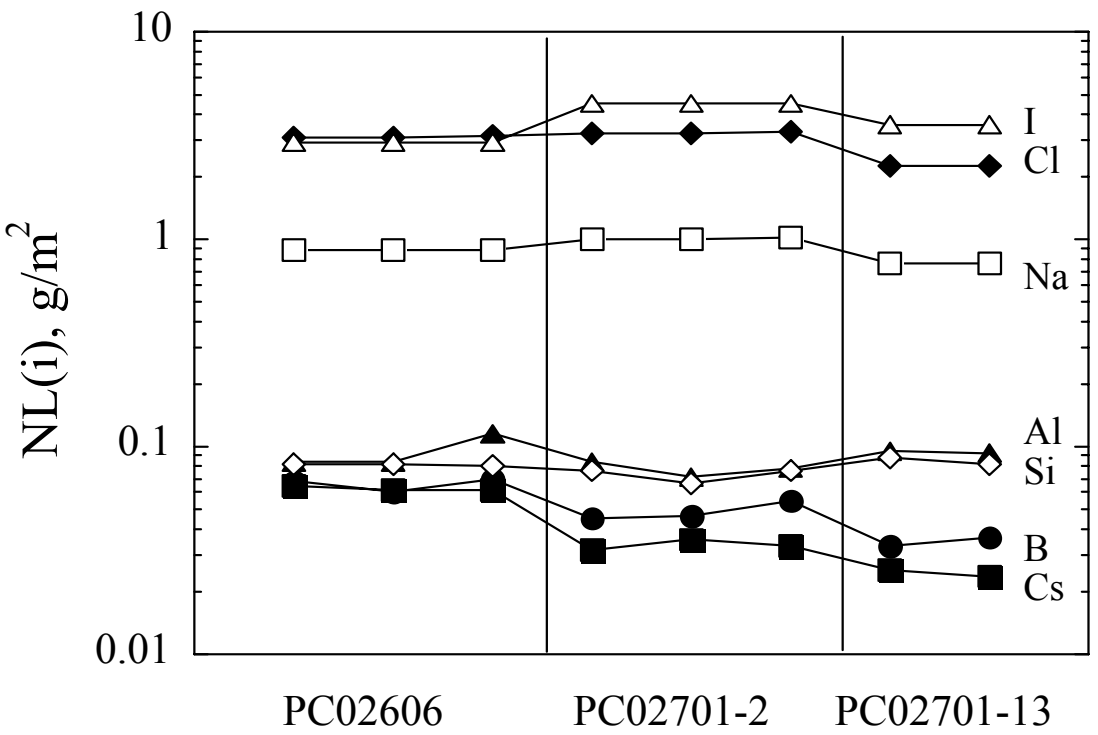

Fig. 11. Summary Plot of Total $N L(i)$ in 7-Day PCTs for Advanced PC CWF Samples. 


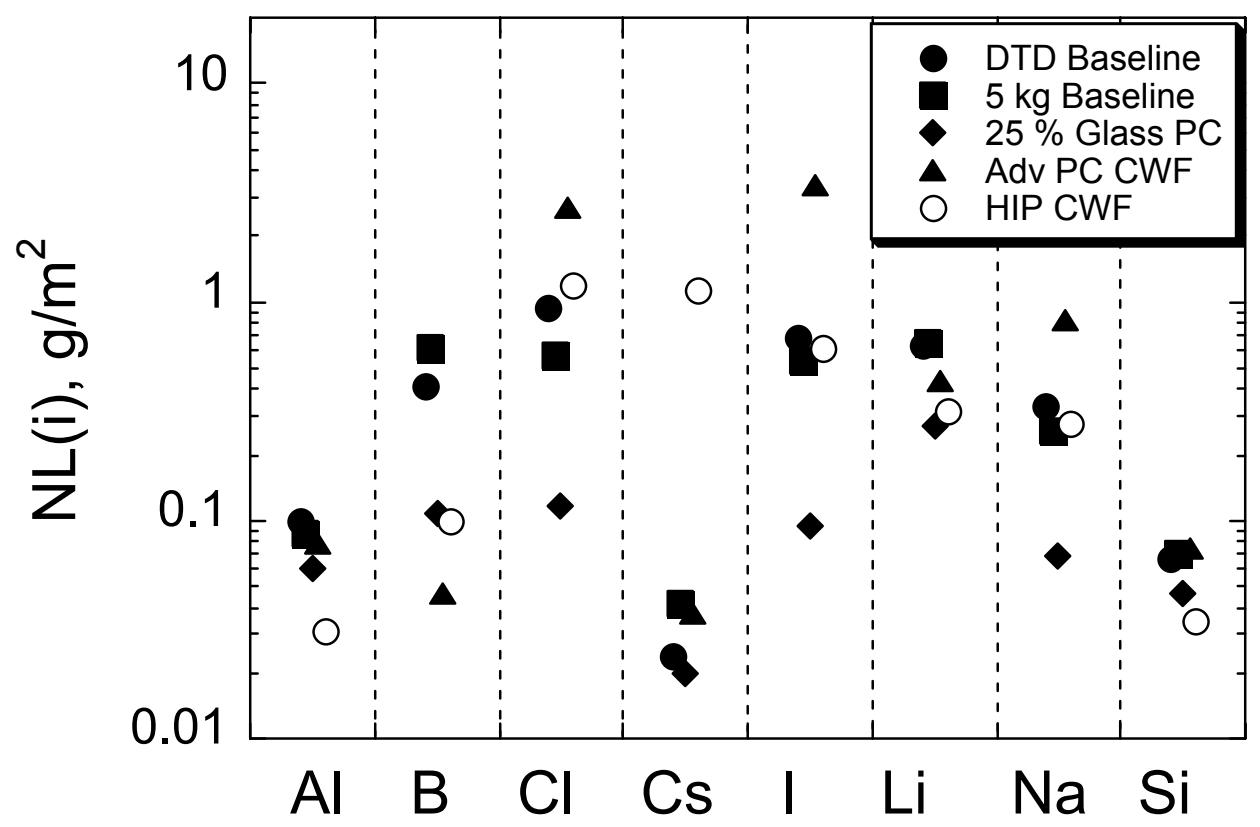

Fig. 12. Total $N L(i)$ in 7-Day PCTs with DTD and 5-kg Baseline PC CWF, 25\% Glass Loading PC CWF, Advanced PC CWF, and HIP CWF Products. 


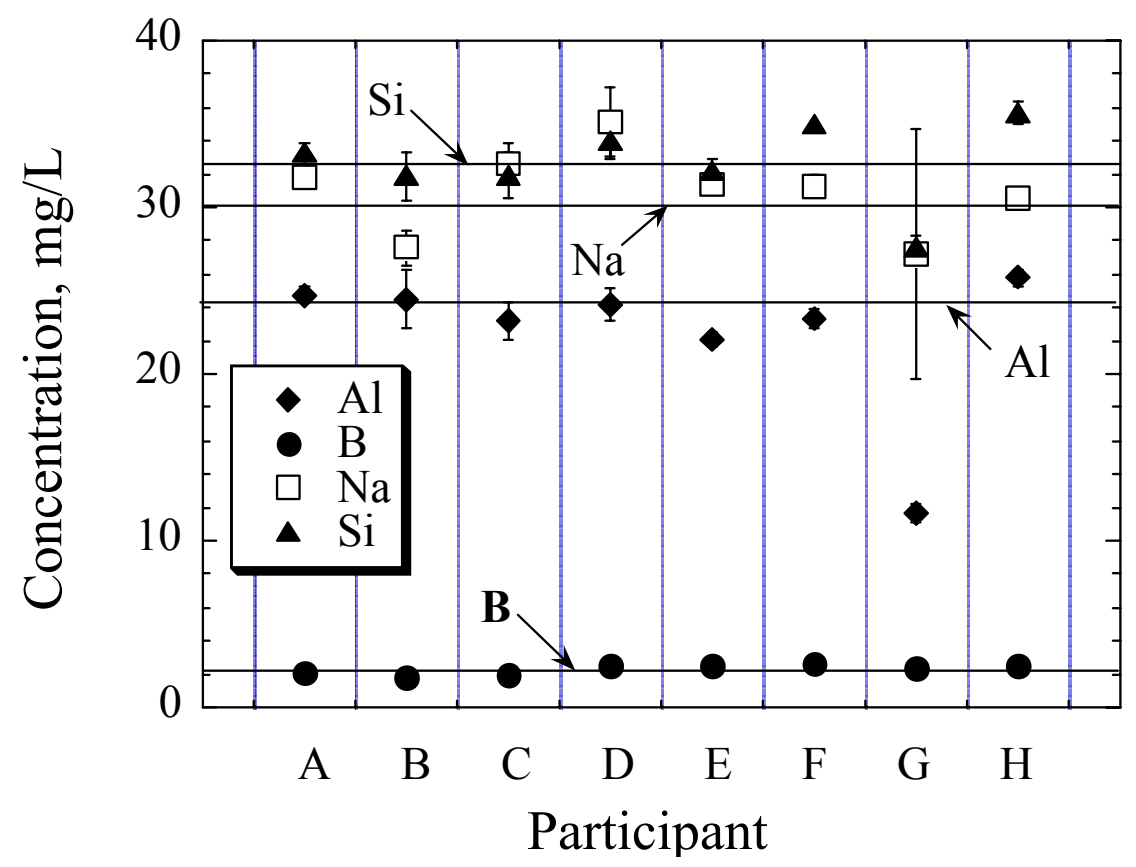

Fig. 13. Mean Values of Solution Concentrations. Lines Show Consensus Means (Excluding Values for Participants D and E) for: $\mathrm{Al}=24.3, \mathrm{~B}=2.25 \mathrm{mg} / \mathrm{L}$, $\mathrm{Na}=30.2 \mathrm{mg} / \mathrm{L}$, and $\mathrm{Si}=32.6 \mathrm{mg} / \mathrm{L}$. 


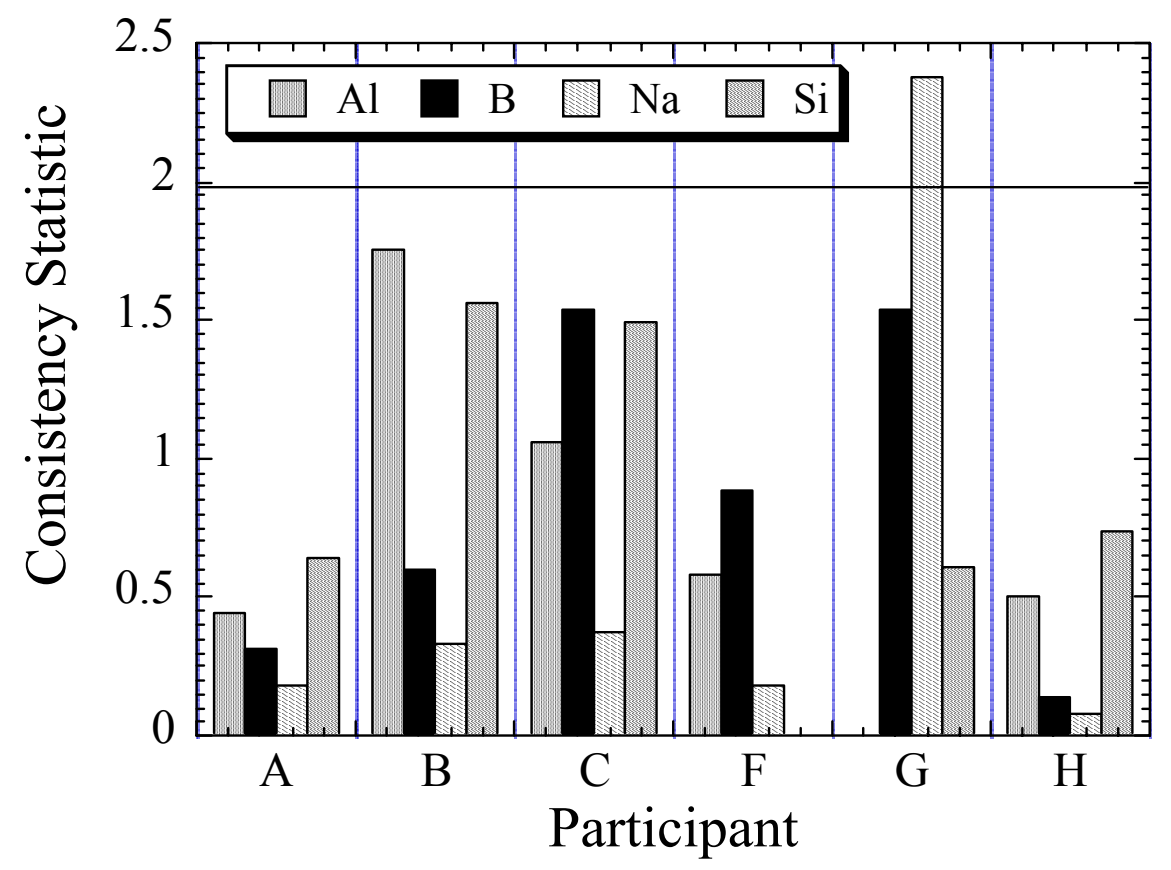

Fig. 14. Plot of $k$ Statistic Values and Critical Value $=1.98$ (for Comparing Intralaboratory Consistency for Six Participants Conducting Triplicate Tests) for PCTs with CWF. 


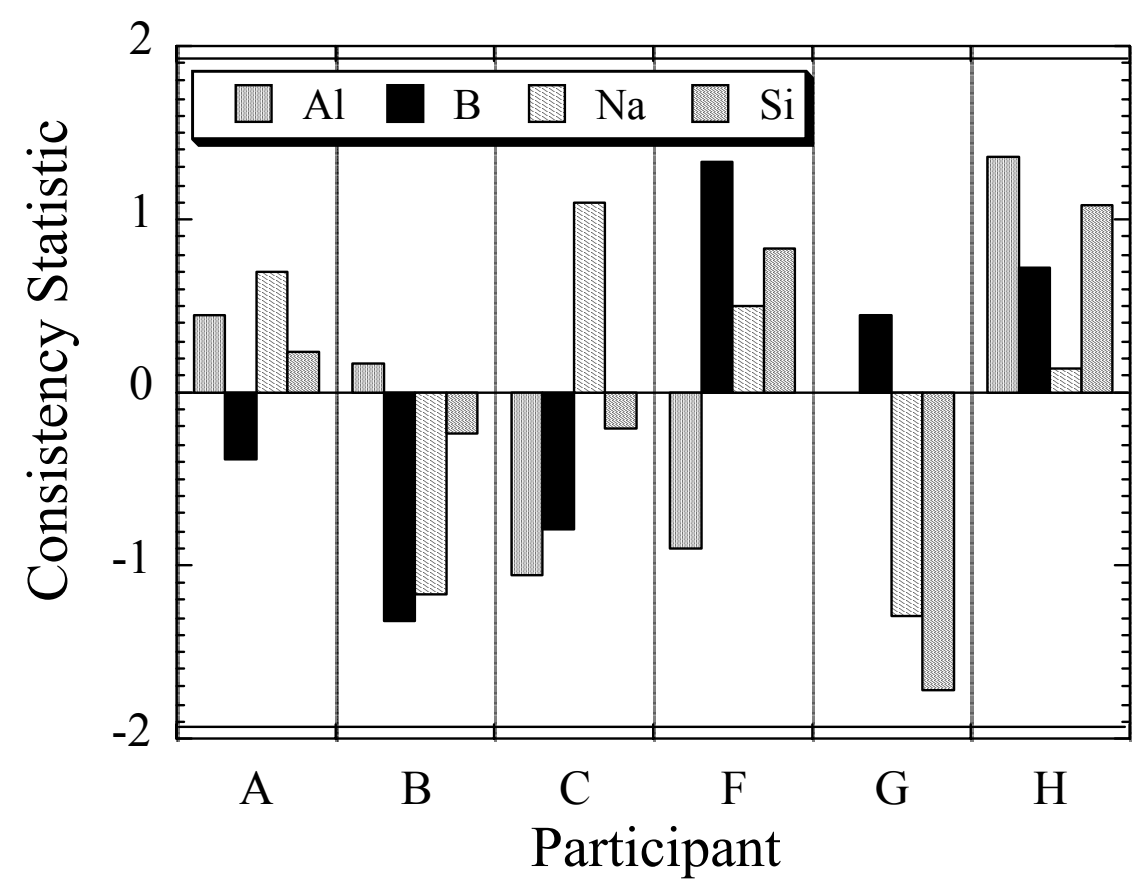

Fig. 15. Plot of $h$ Statistic Values and Critical Value $= \pm 1.92$ (for Comparing Interlaboratory Consistency for Six Participants) for PCTs with CWF. 


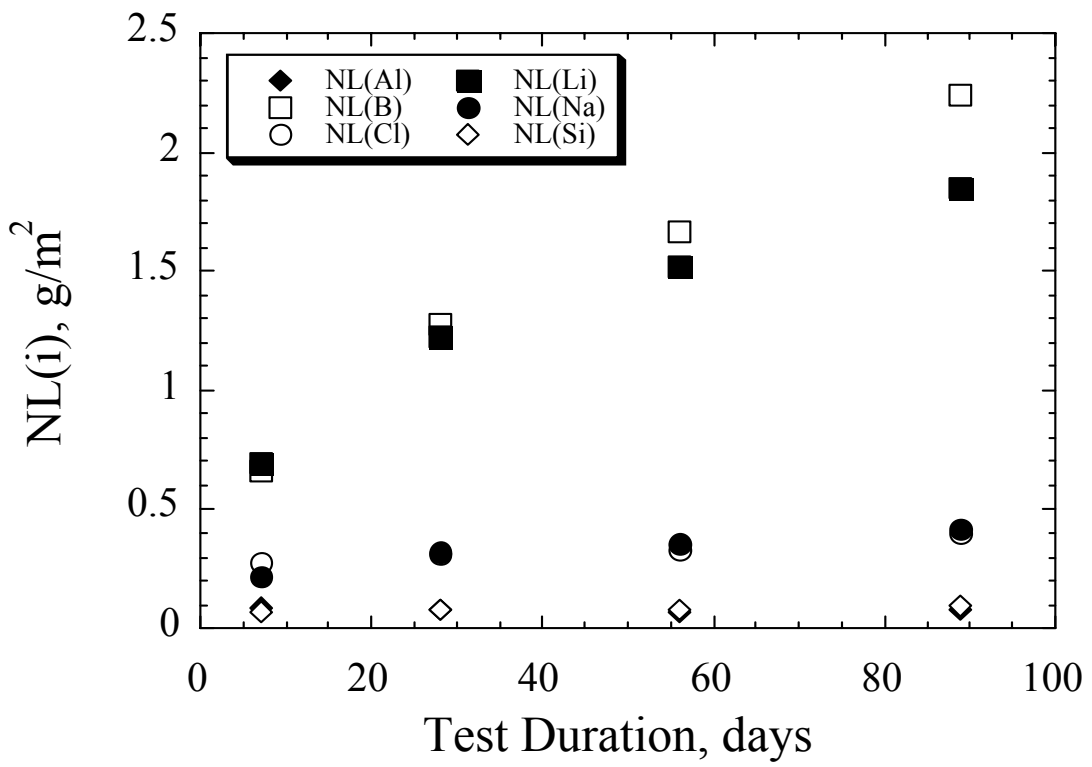

Fig. 16. Mean $N L(i)$ for PCT Fractions of Long-Term PCTs with 5-kg Baseline PC CWF. 


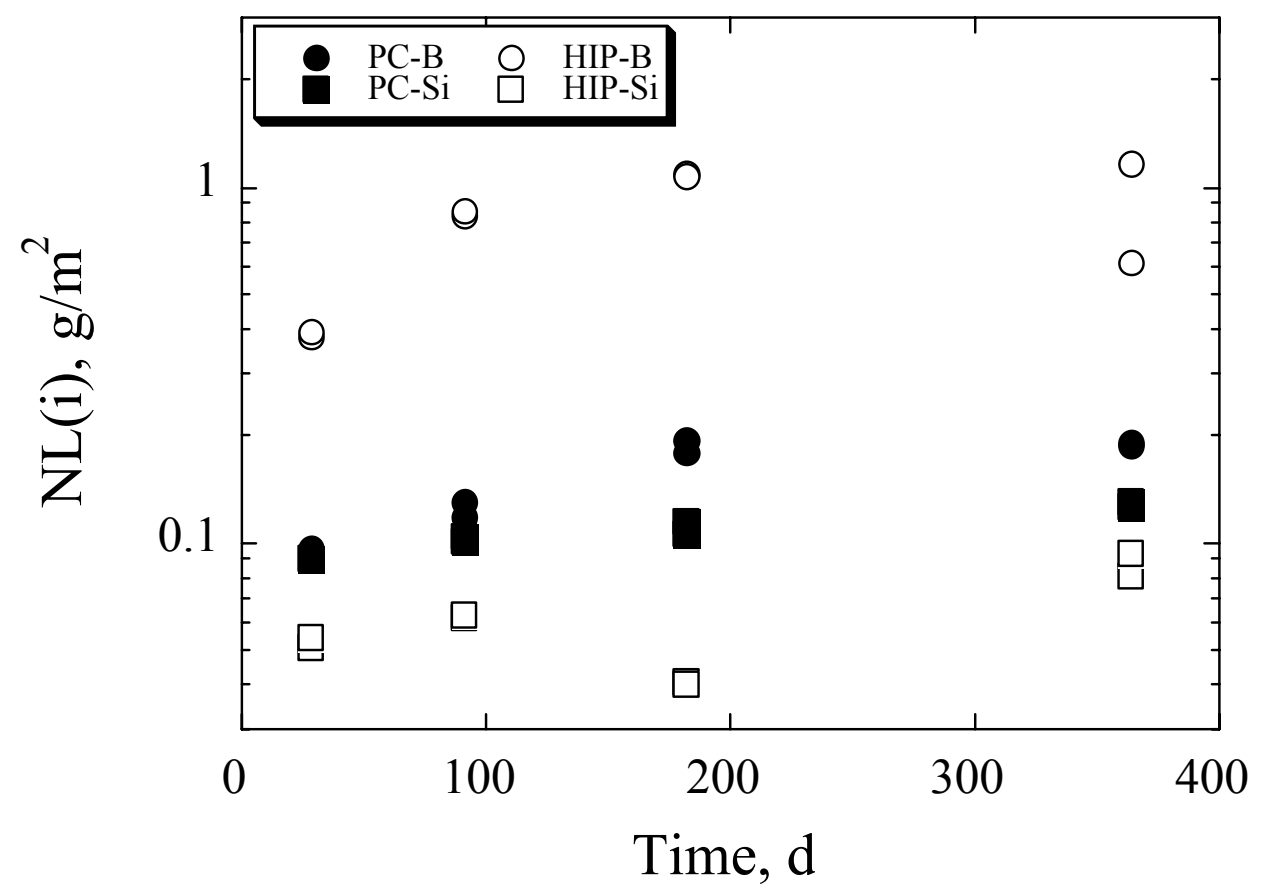

(a)

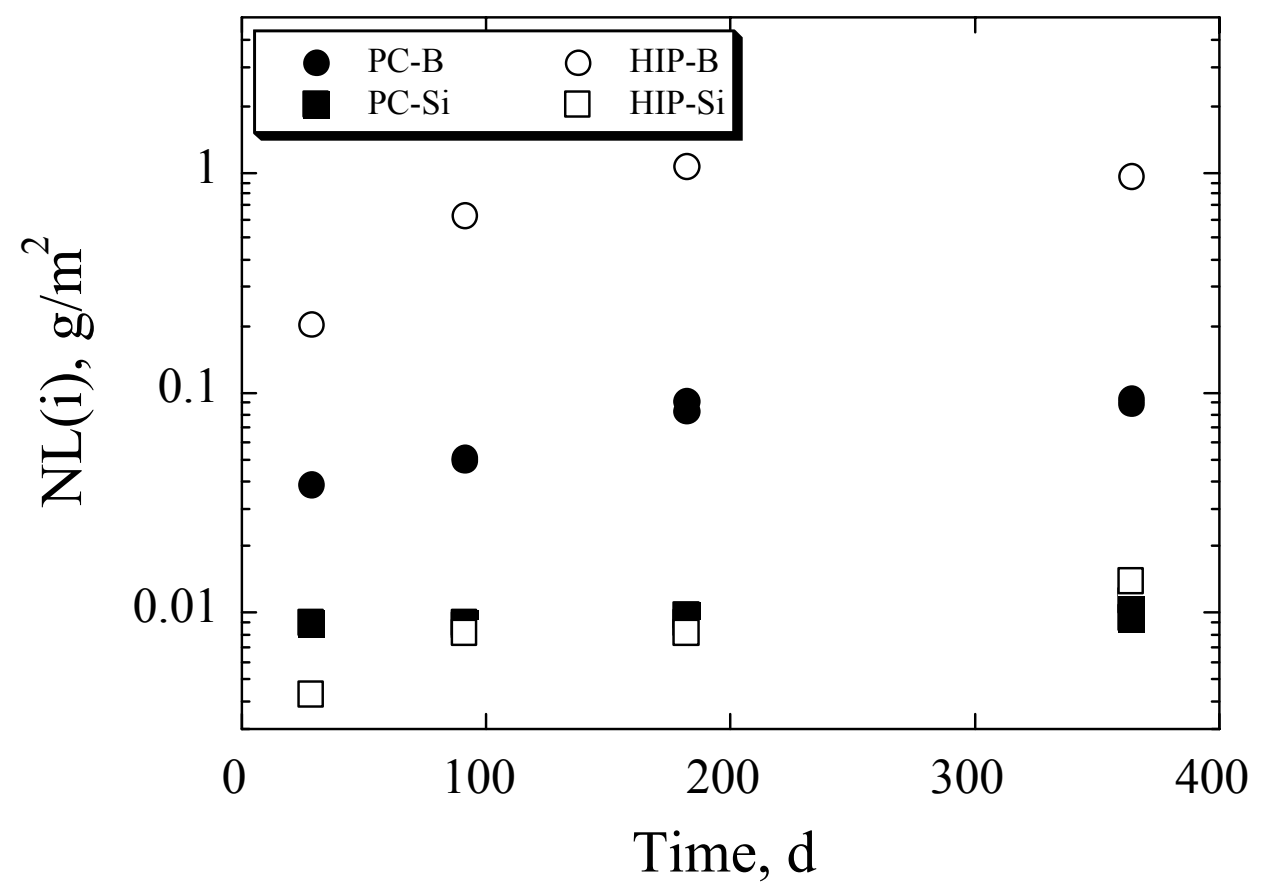

(b)

Fig. 17. $N L(i)$ for Long-Term Tests with Advanced PC CWF and HIP CWF: $N L(\mathrm{~B})$ and $N L(\mathrm{Si})$ in Tests at (a) $2300 \mathrm{~m}^{-1}$ and (b) $23,000 \mathrm{~m}^{-1}$, and $N L(\mathrm{Cl})$ and $N L(\mathrm{Na})$ for Tests at (c) $2300 \mathrm{~m}^{-1}$ and (d) $23,000 \mathrm{~m}^{-1}$. 


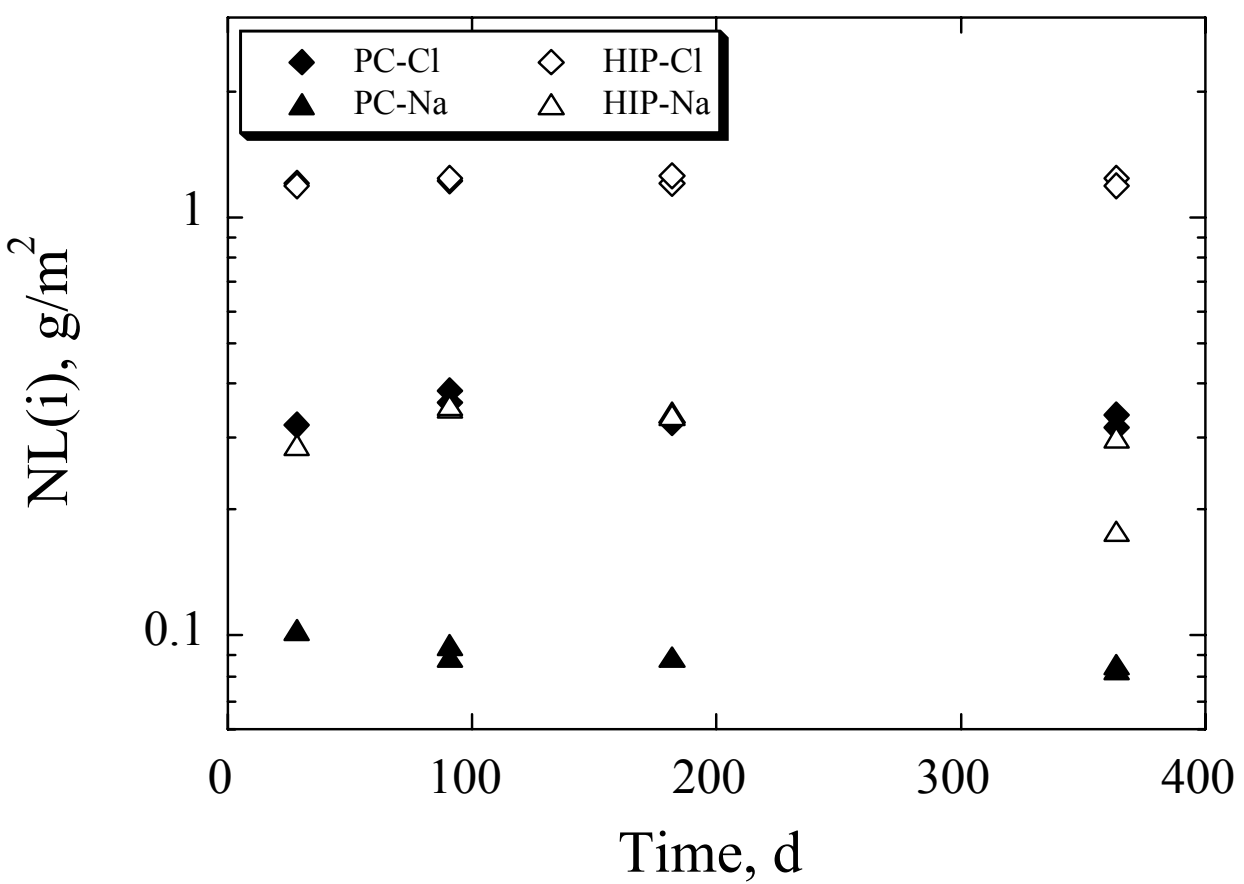

(c)

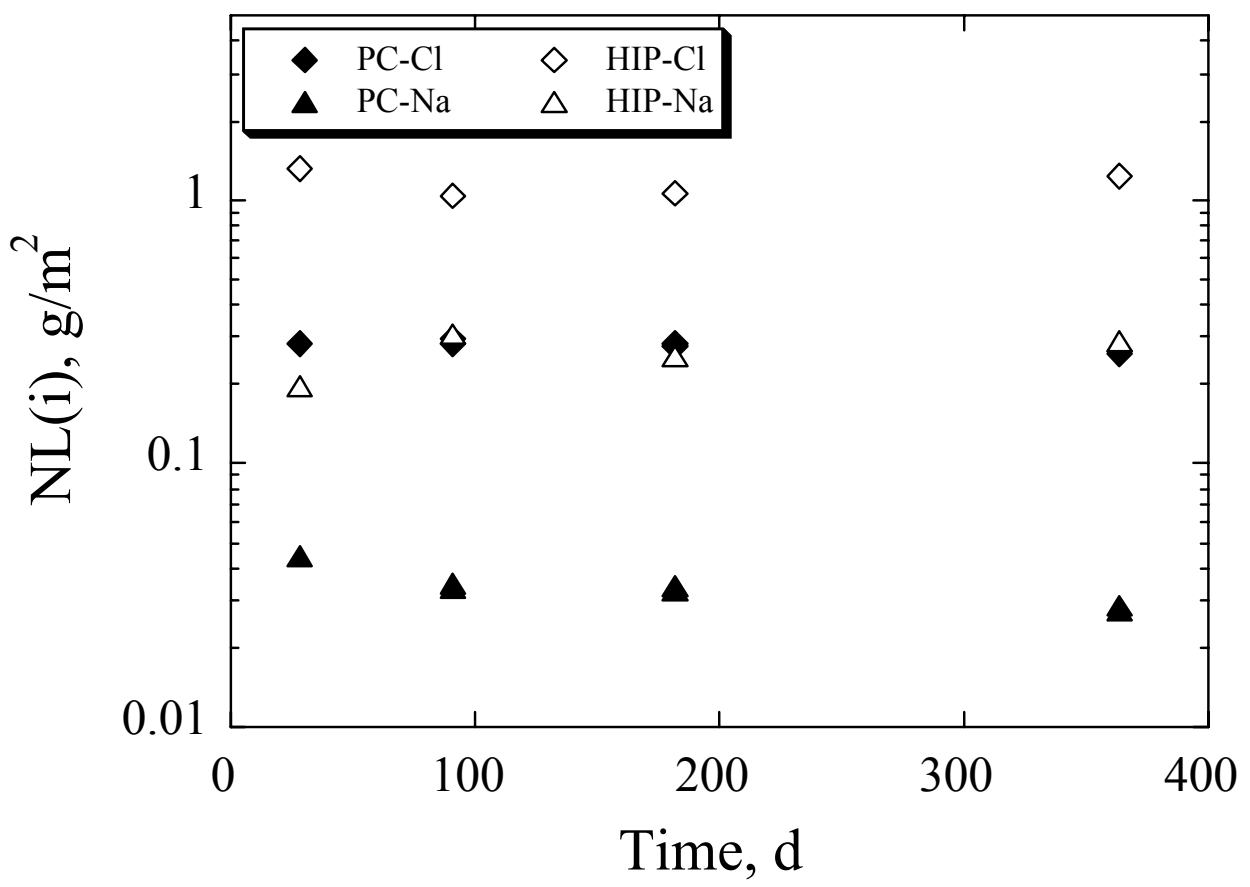

(d)

Fig. 17. (Contd). 


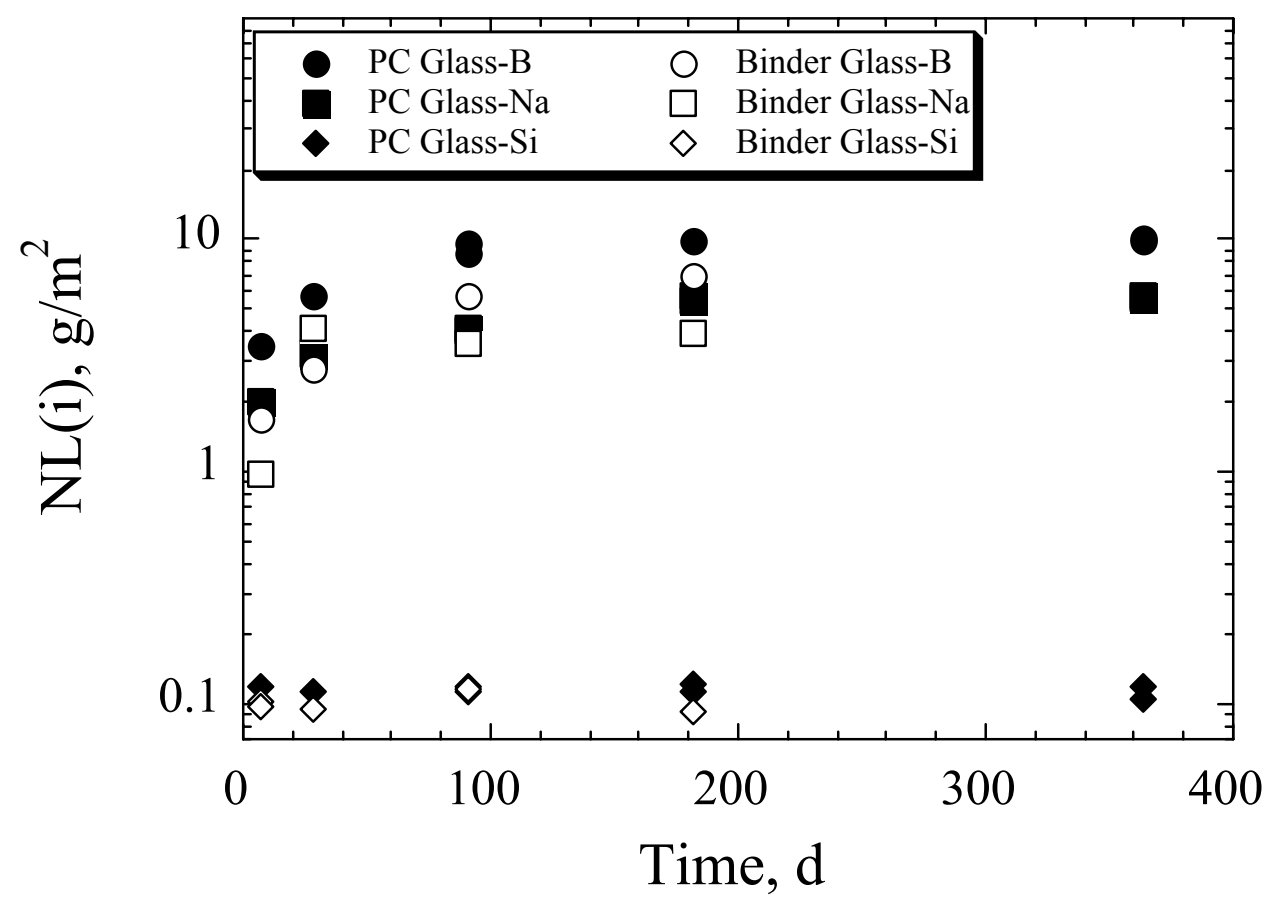

(a)

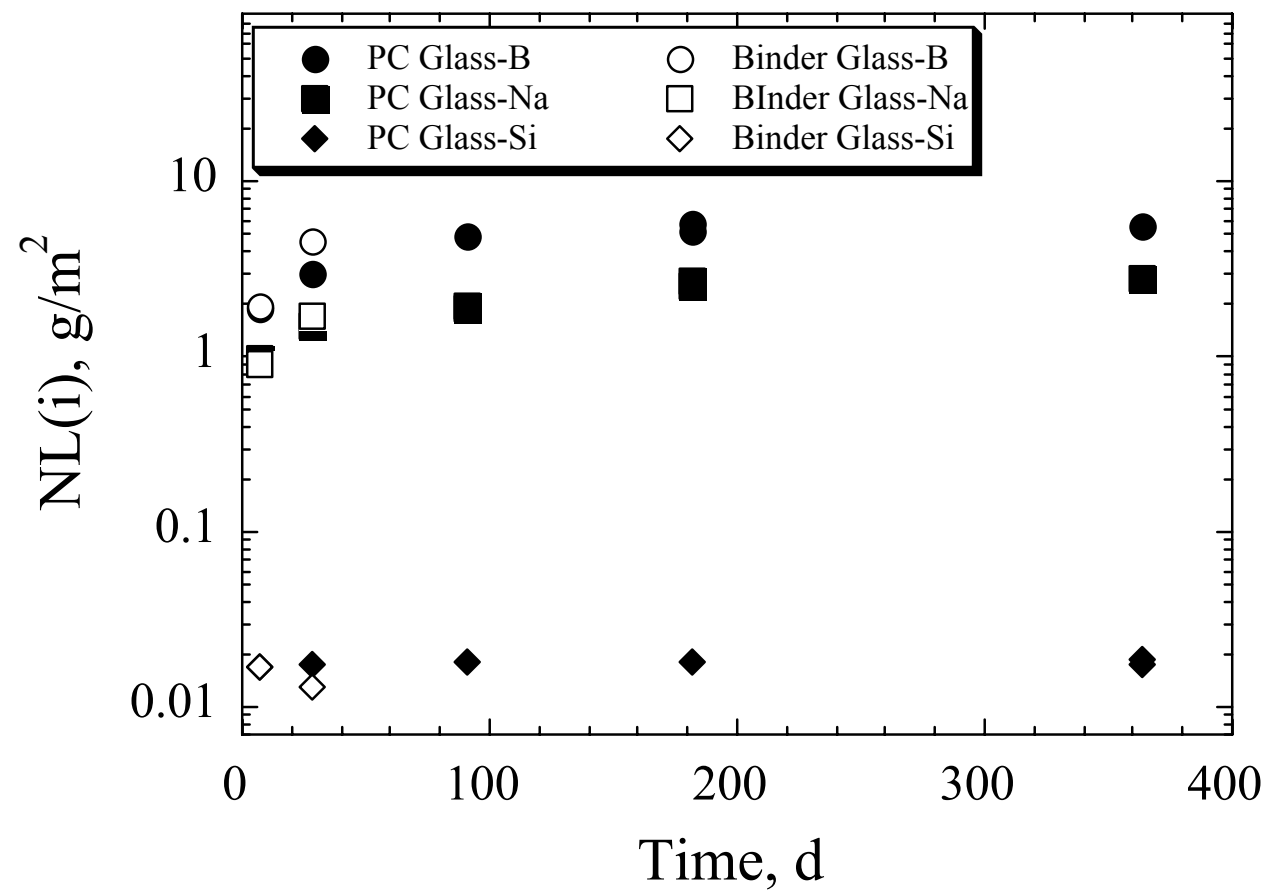

(b)

Fig. 18. $N L(i)$ for Long-Term Tests with PC Glass and Binder Glass: $N L(\mathrm{~B}), N L(\mathrm{Na})$, and $N L(\mathrm{Si})$ in Tests at (a) $2300 \mathrm{~m}^{-1}$ and (b) $23,000 \mathrm{~m}^{-1}$. 


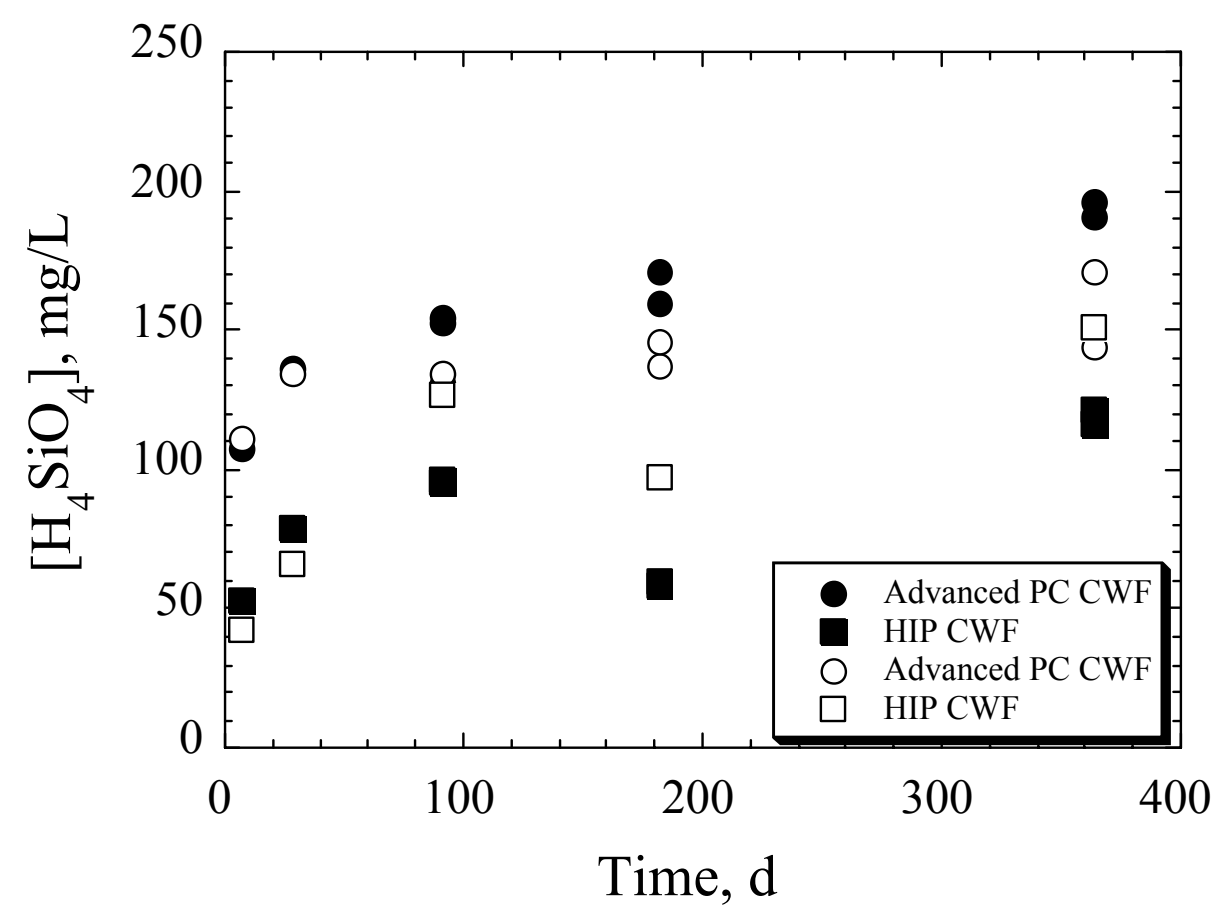

(a)

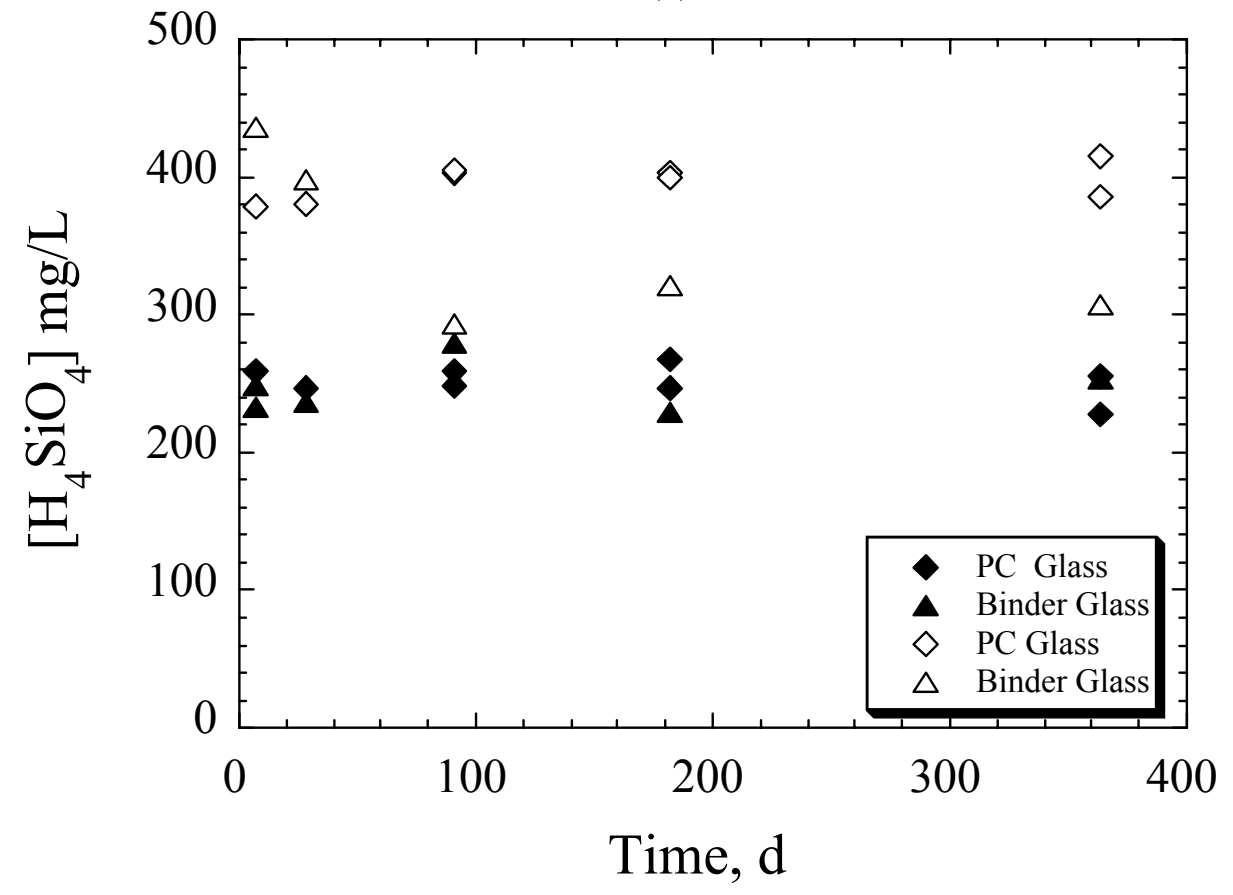

(b)

Fig. 19. Orthosilicic Acid Concentrations in PCT with (a) Advanced PC CWF and HIP CWF, and (b) PC Glass and Binder Glass. Filled Symbols for Tests Conducted at $2300 \mathrm{~m}^{-1}$ and Open Symbols for Tests Conducted at 23,000 $\mathrm{m}^{-1}$. 

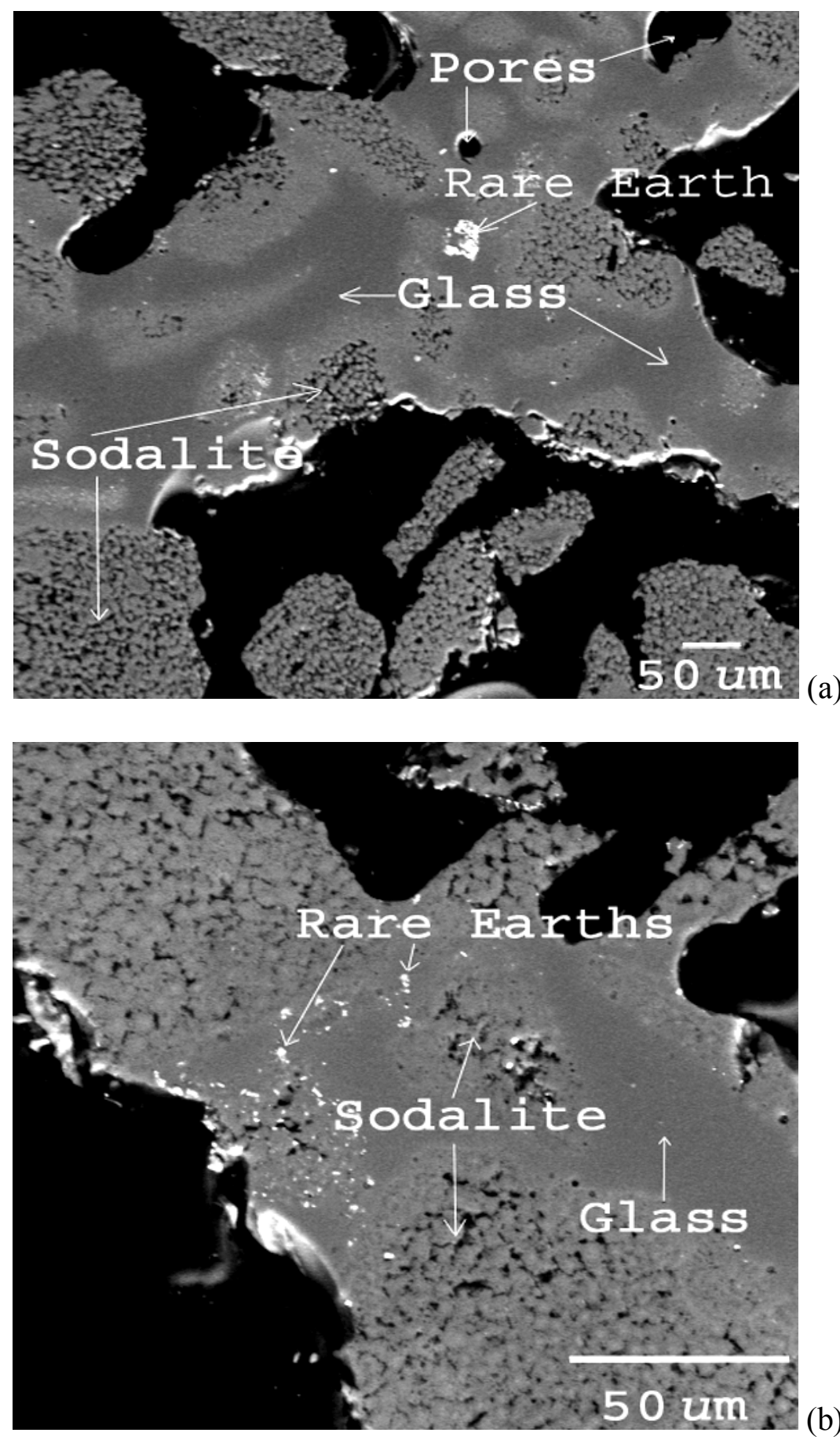

(b)

Fig. 20. SEM Image of T-t PC CWF Material NLS-2 Processed at $850^{\circ} \mathrm{C}$ with 1 -h Hold Time at (a) Moderate Magnification and (b) High Magnification. 


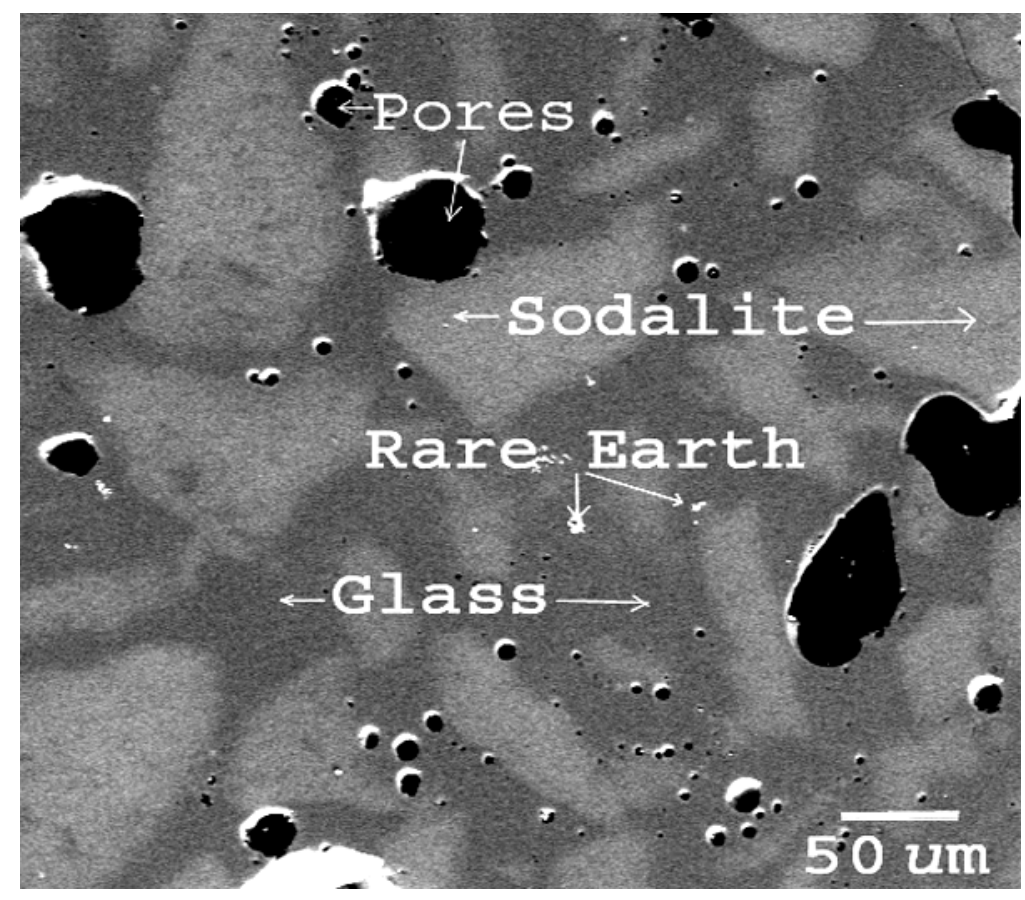

Fig. 21. SEM Image of T-t PC CWF Material NLS-8 Processed at $915^{\circ} \mathrm{C}$ with $16-\mathrm{h}$ Hold Time. 


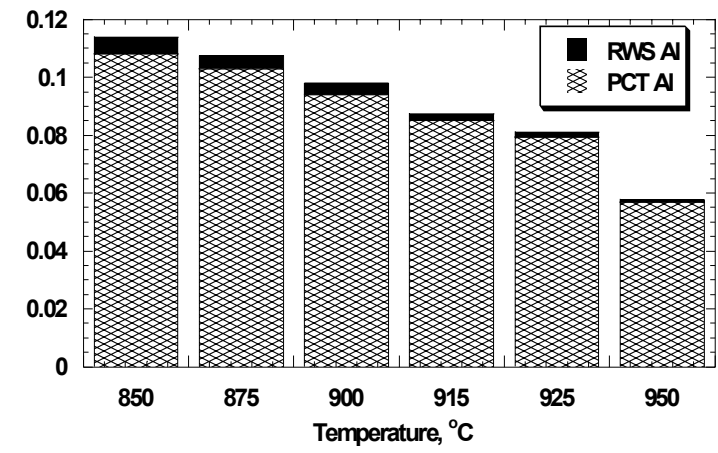

(a)

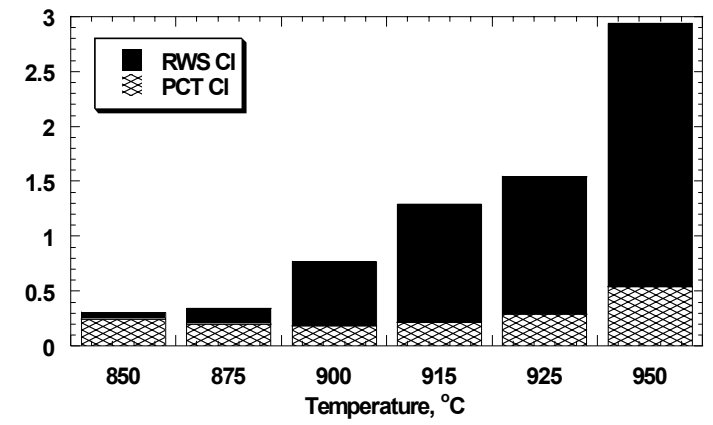

(c)

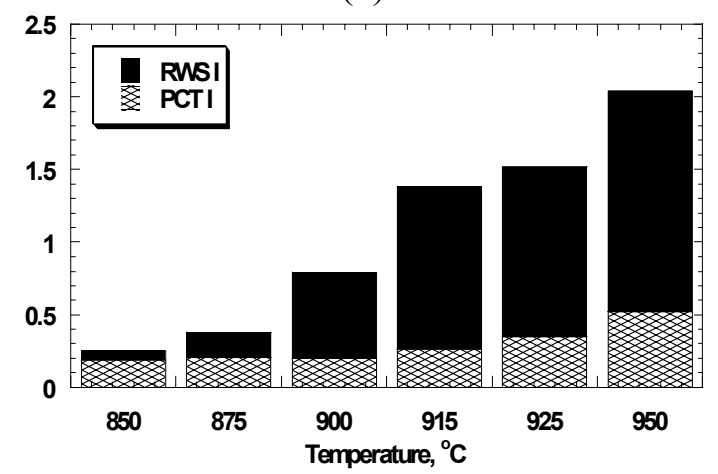

(e)

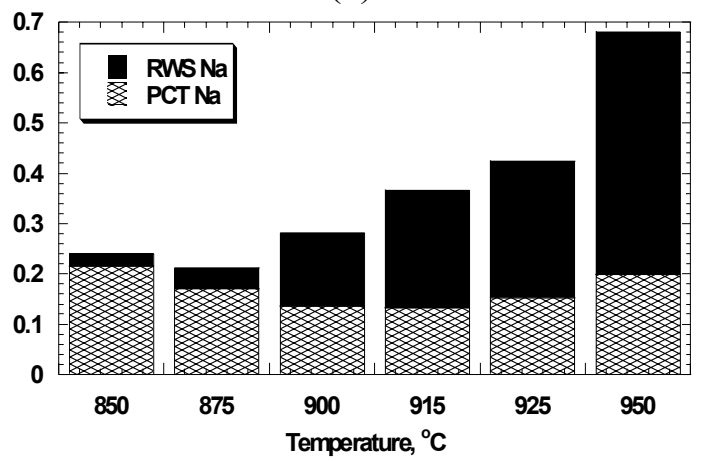

(g)

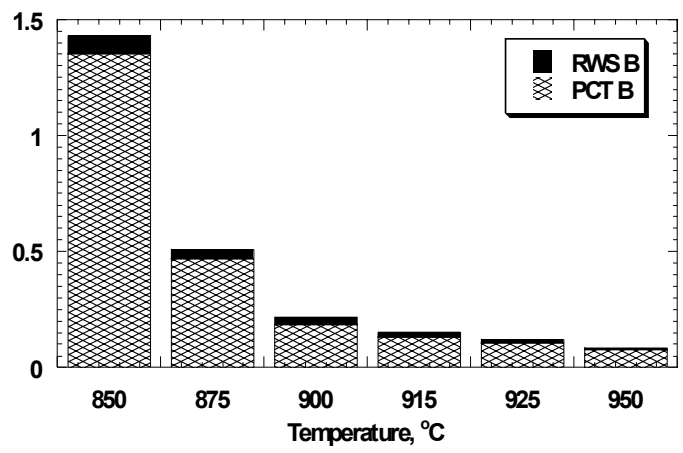

(b)

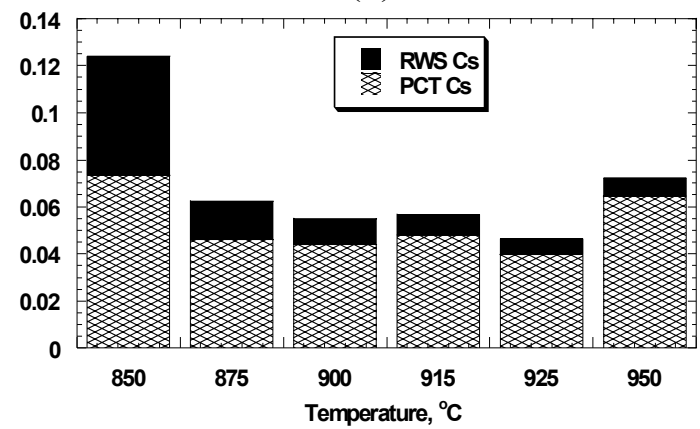

(d)

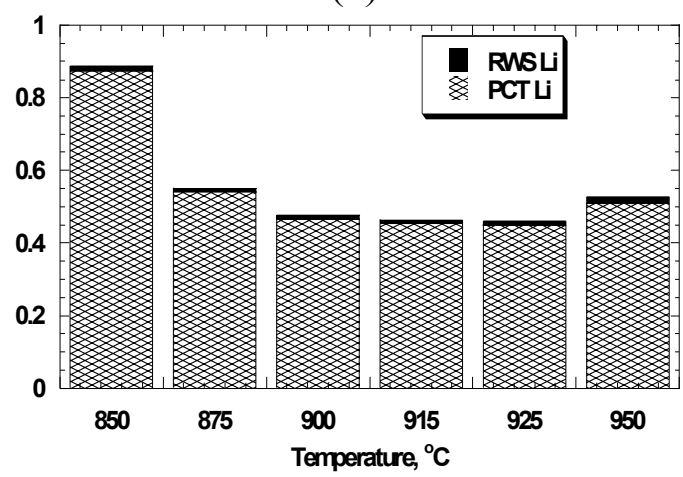

(f)

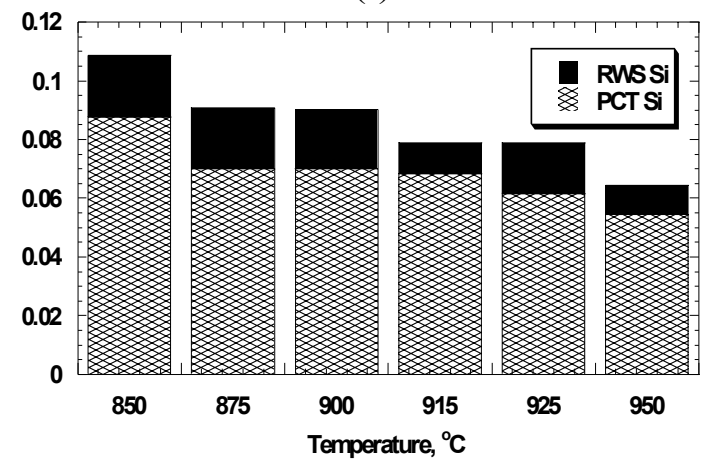

(h)

Fig. 22. $\quad N L(i)$ Values for 7-Day PCTs with T-t PC CWF Made with 1-h Hold Time for $\mathrm{i}=$ (a) Al, (b) B, (c) Cl, (d) Cs, (e) I, (f) Li, (g) Na, and (h) Si. 


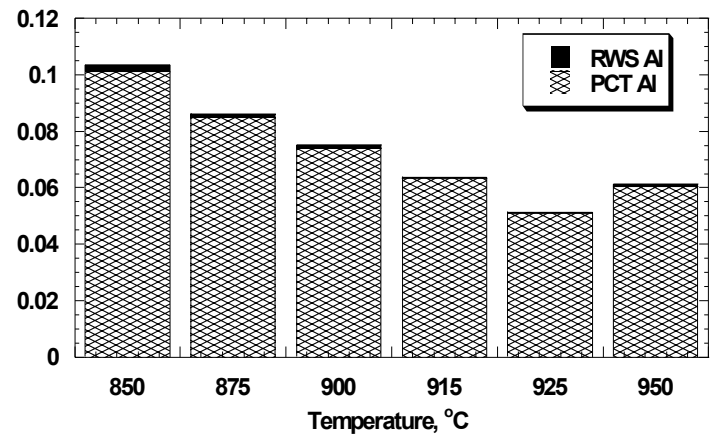

(a)

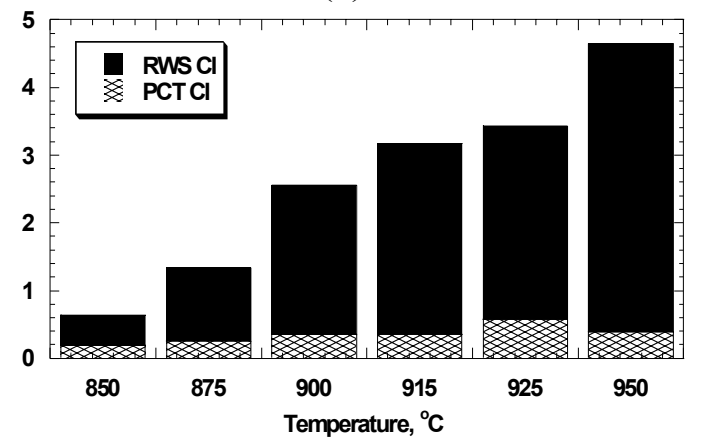

(c)

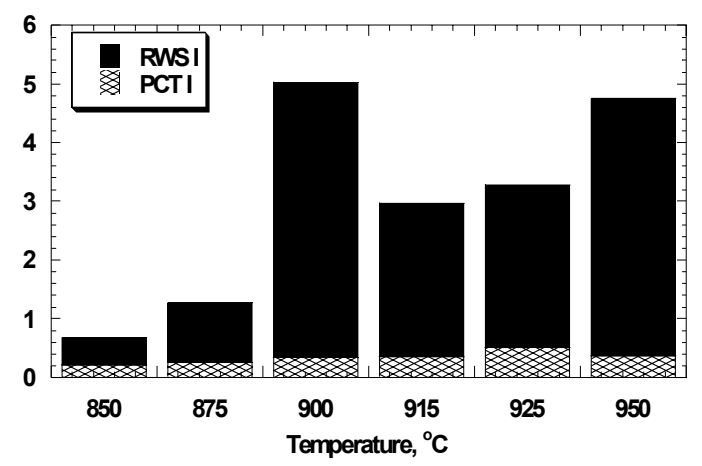

(e)

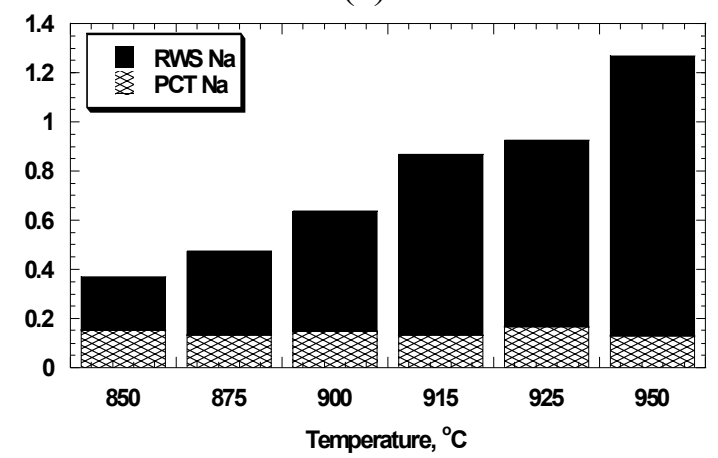

(g)

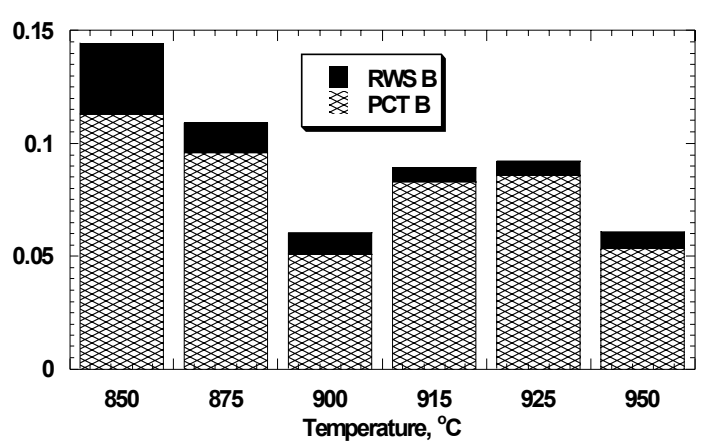

(b)

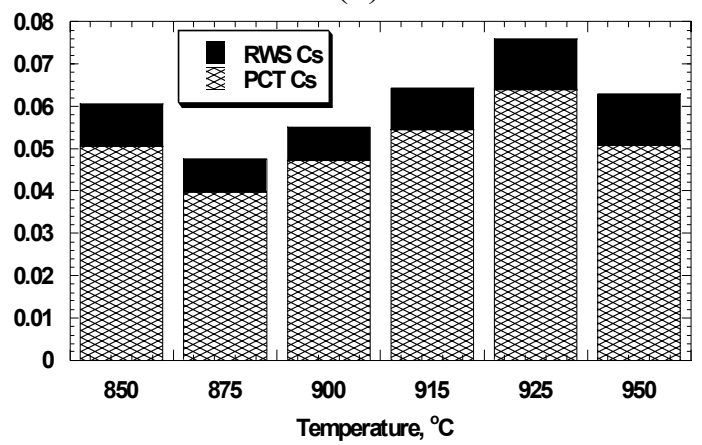

(d)

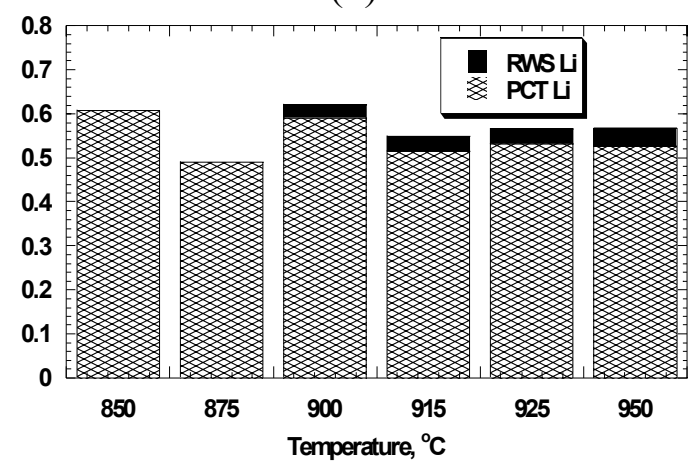

(f)

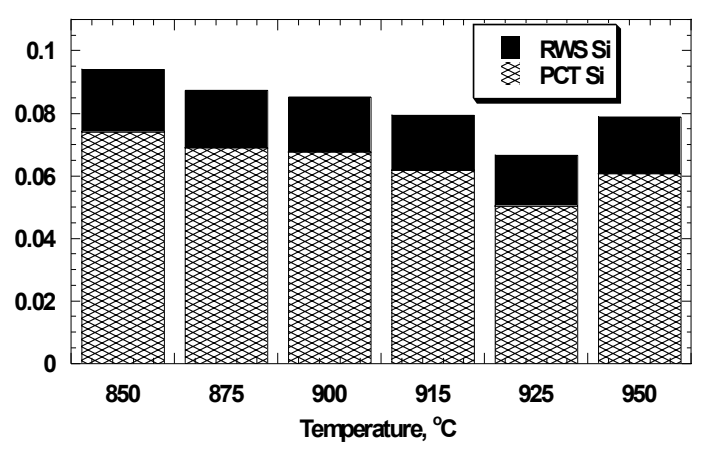

(h)

Fig. 23. $\quad N L(i)$ Values for 7-Day PCTs with T-t PC CWF Made with 4-h Hold Time for $\mathrm{i}=$ (a) Al, (b) B, (c) Cl, (d) Cs, (e) I, (f) Li, (g) Na, and (h) Si. 


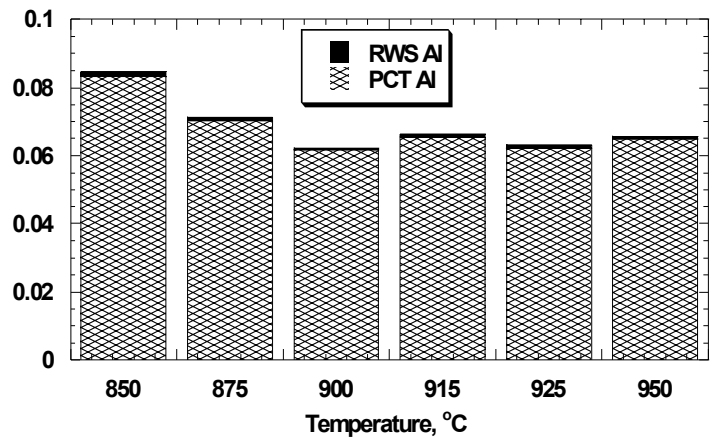

(a)

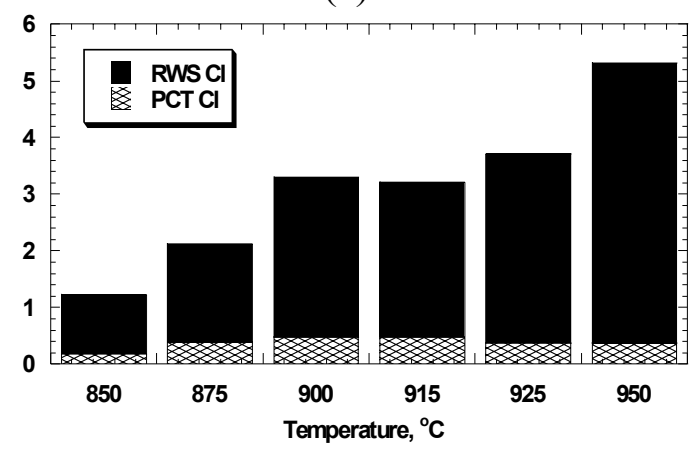

(c)

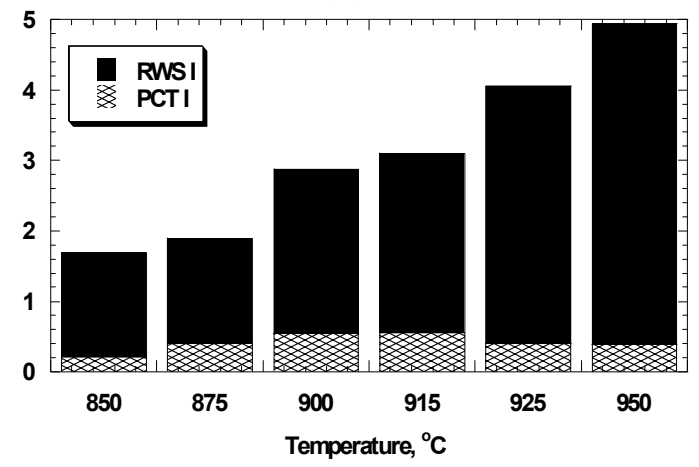

(e)

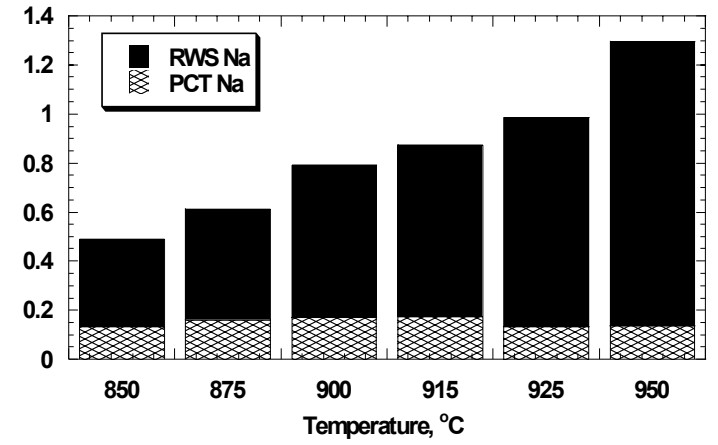

(g)

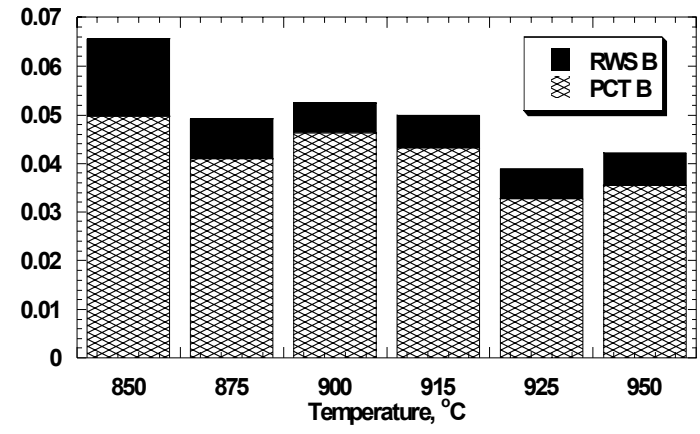

(b)

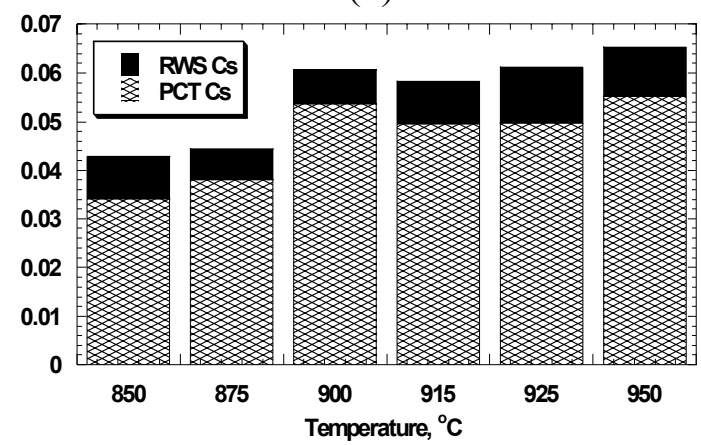

(d)

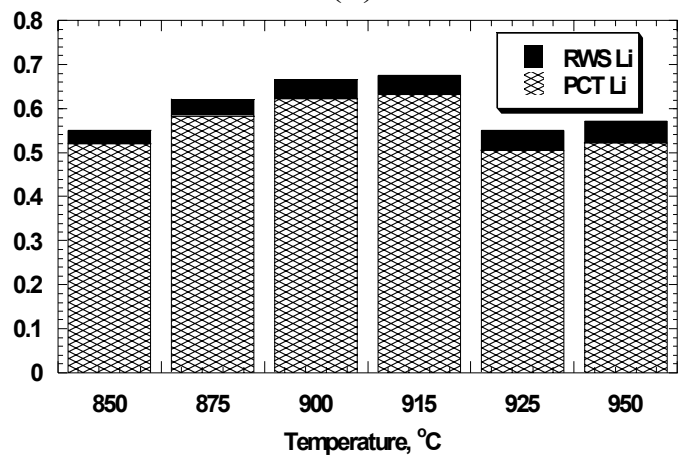

(f)

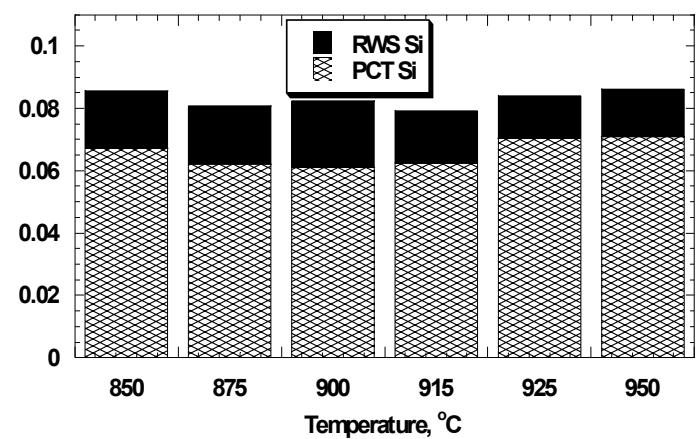

(h)

Fig. 24. $\quad N L(i)$ Values for 7-Day PCTs with T-t PC CWF Made with 8-h Hold Time for $\mathrm{i}=$ (a) Al, (b) B, (c) Cl, (d) Cs, (e) I, (f) Li, (g) Na, and (h) Si. 


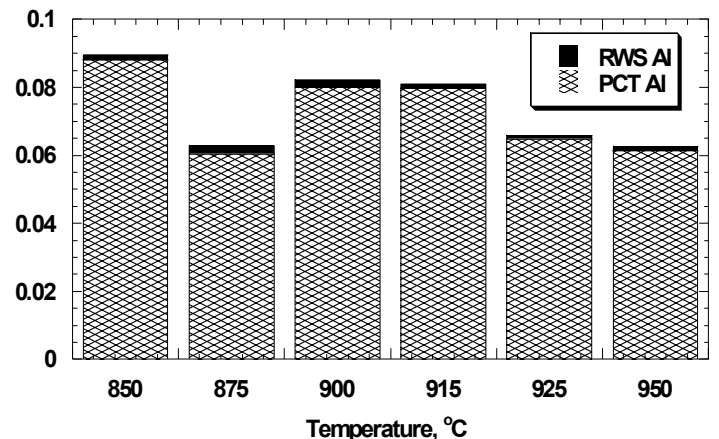

(a)

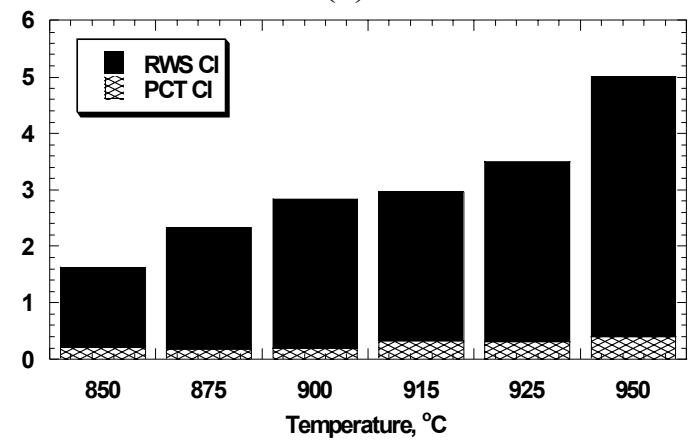

(c)

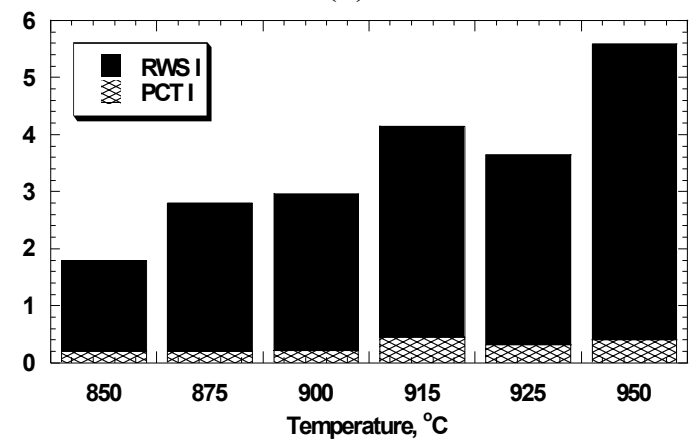

(e)

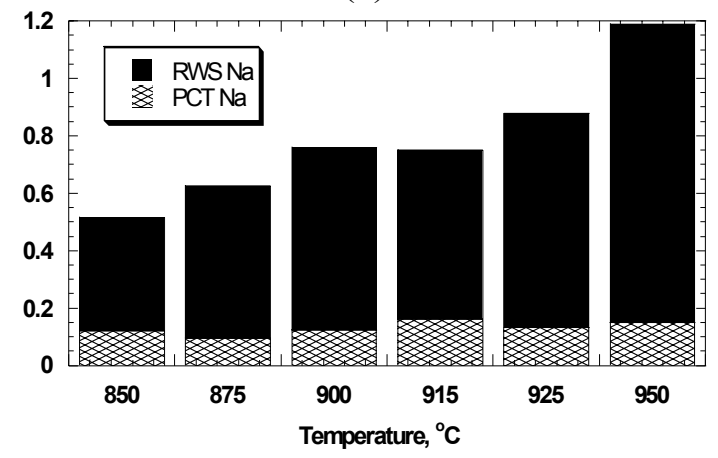

(g)

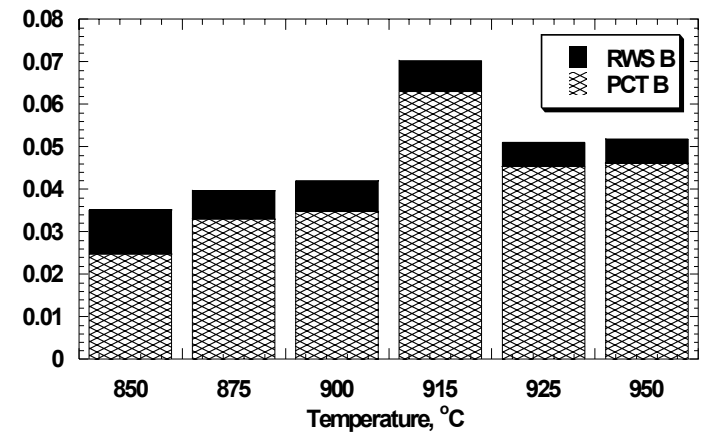

(b)

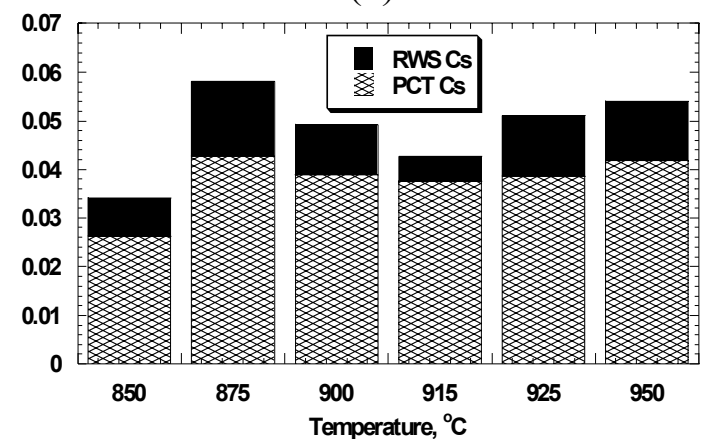

(d)

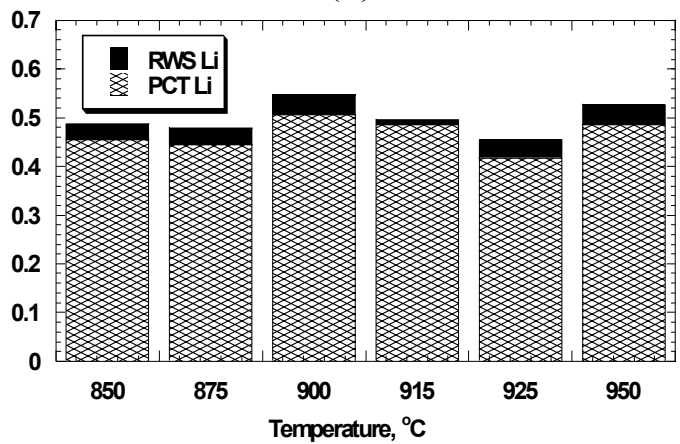

(f)

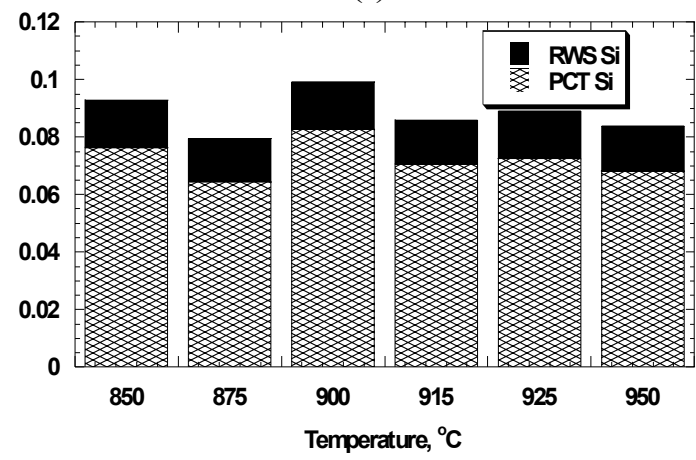

(h)

Fig. 25. $\quad N L(i)$ Values for 7-Day PCTs with T-t PC CWF Made with 16-h Hold Time for i = (a) Al, (b) B, (c) Cl, (d) Cs, (e) I, (f) Li, (g) Na, and (h) Si. 


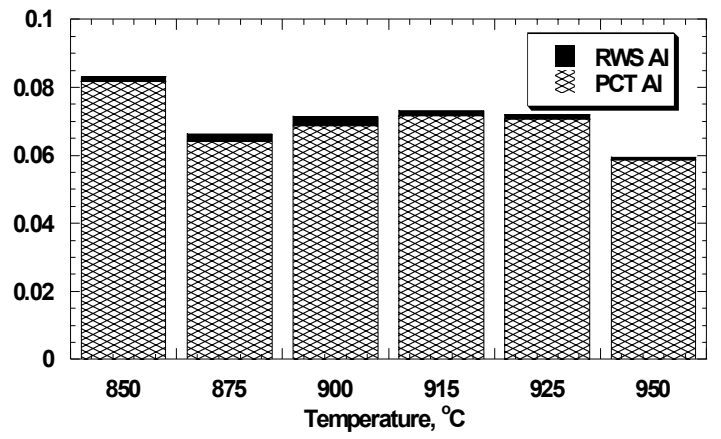

(a)

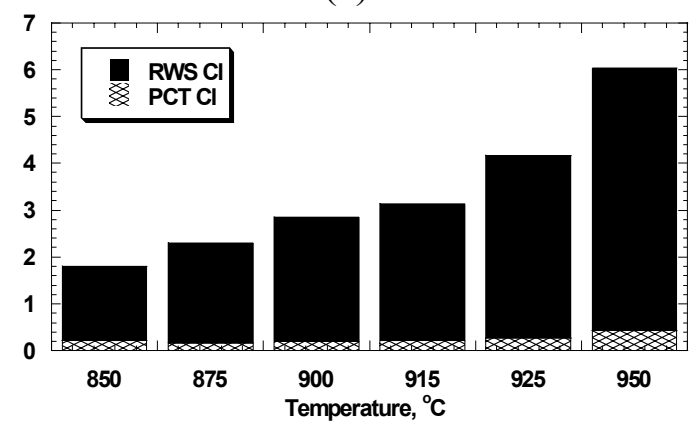

(c)

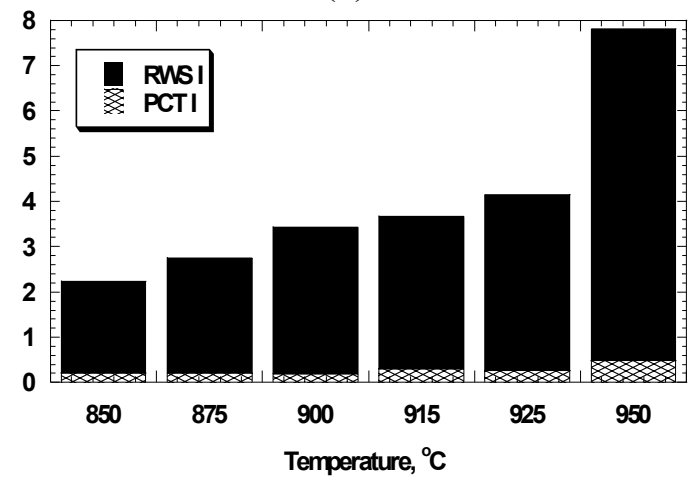

(e)

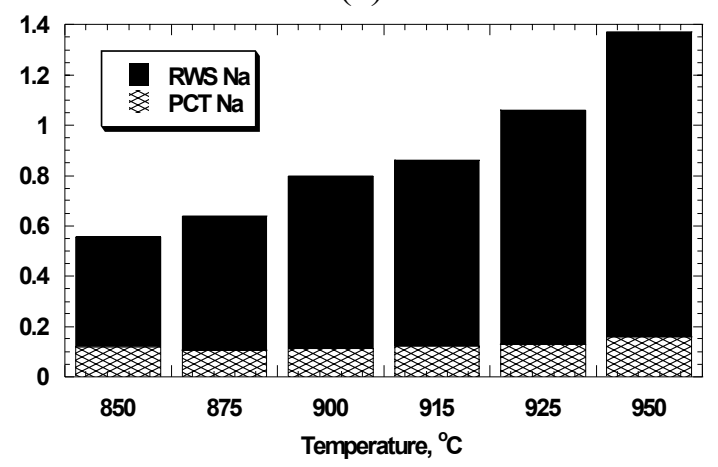

(g)

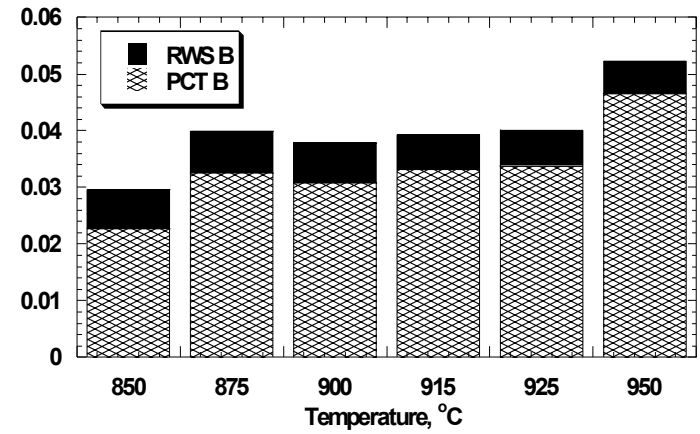

(b)

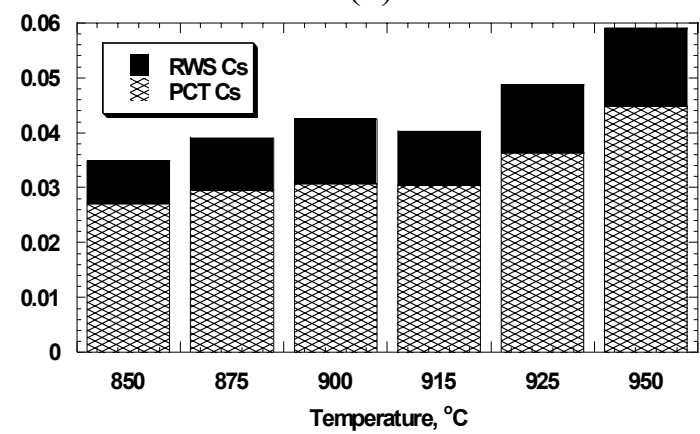

(d)

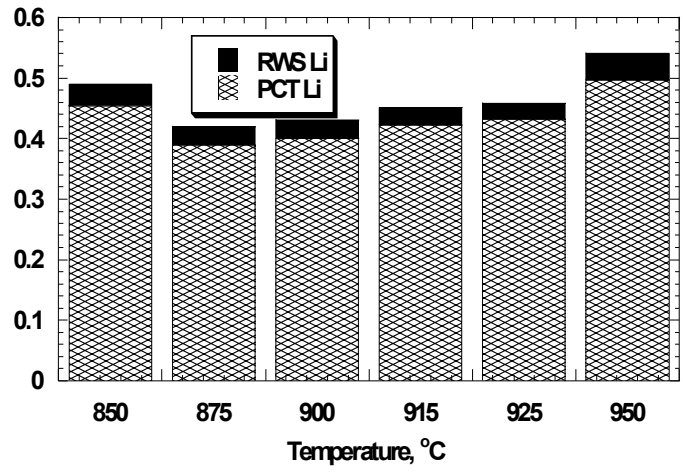

(f)

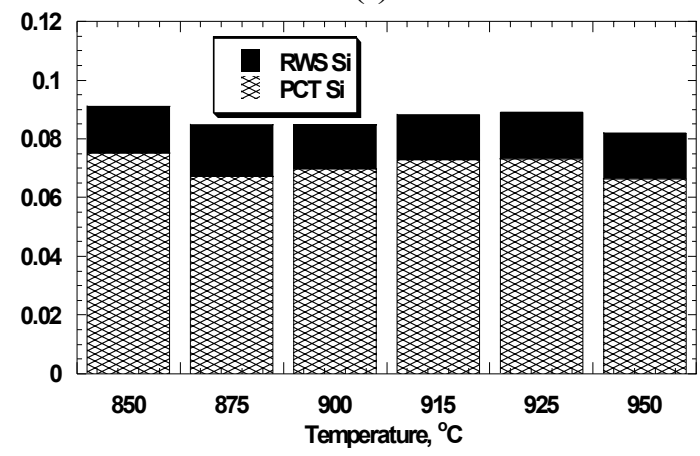

(h)

Fig. 26. $\quad N L(i)$ Values for 7-Day PCTs with T-t PC CWF Made with 4-h Hold Time for $\mathrm{i}=$ (a) Al, (b) B, (c) Cl, (d) Cs, (e) I, (f) Li, (g) Na, and (h) Si. 


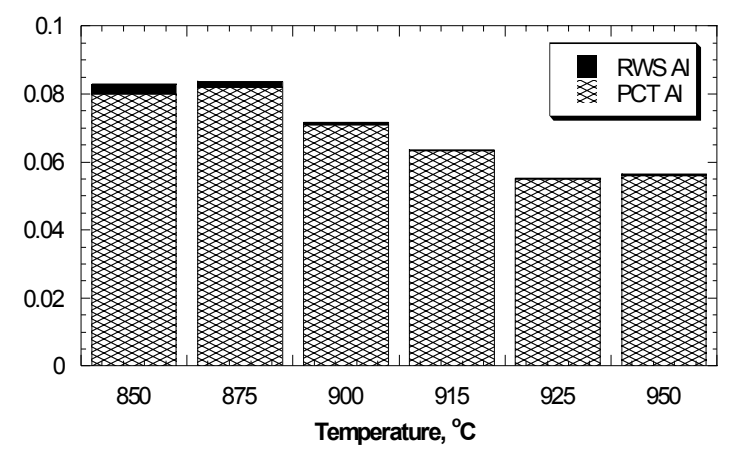

(a)

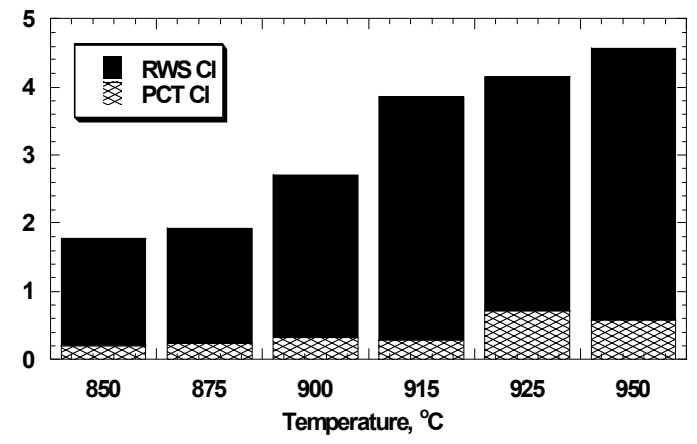

(c)

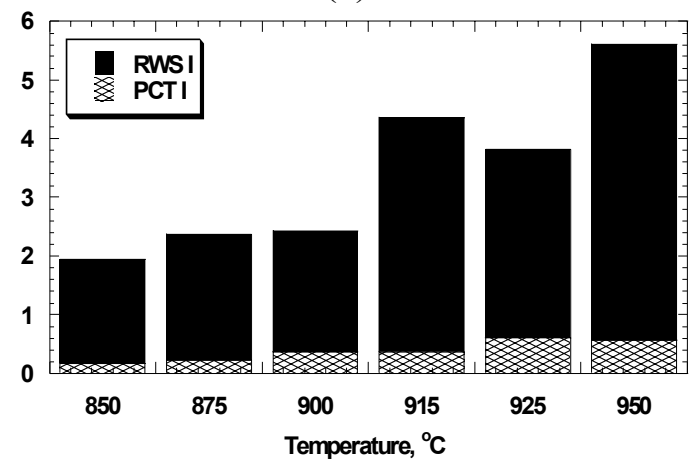

(e)

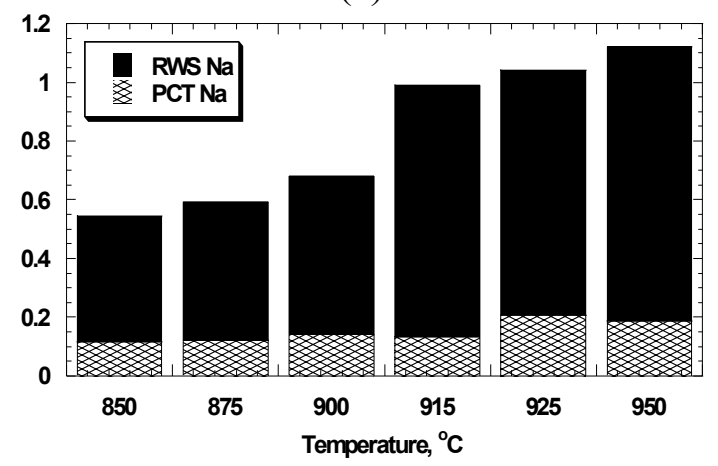

(g)

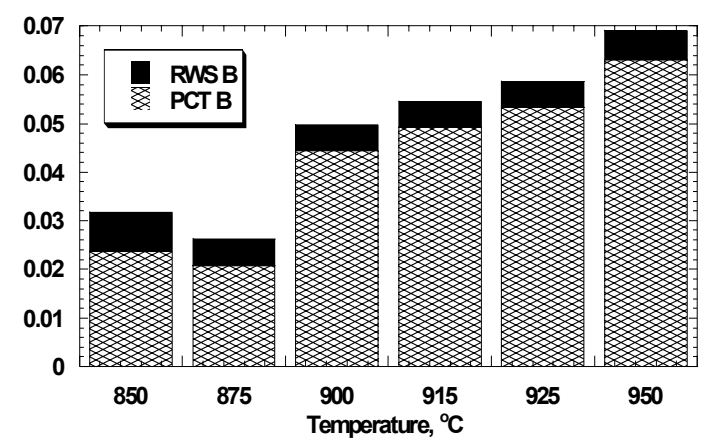

(b)

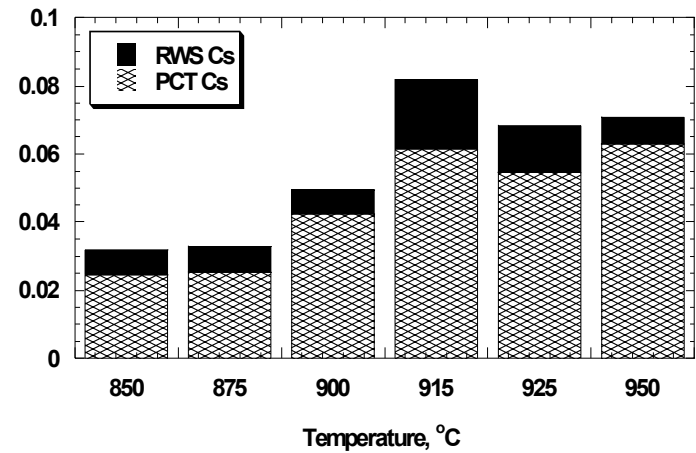

(d)

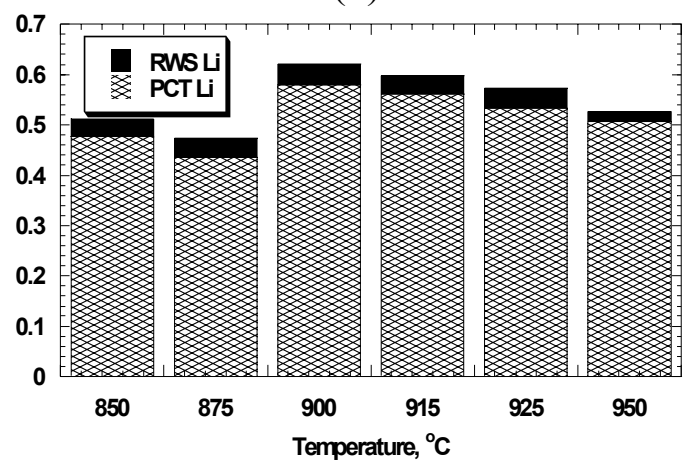

(f)

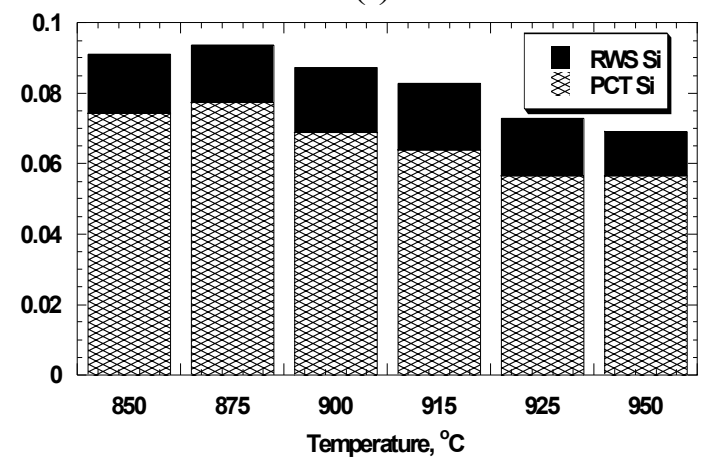

(h)

Fig. 27. $\quad N L(i)$ Values for 7-Day PCTs with T-t PC CWF Made with 36-h Hold Time for $\mathrm{i}=$ (a) Al, (b) B, (c) Cl, (d) Cs, (e) I, (f) Li, (g) Na, and (h) Si. 


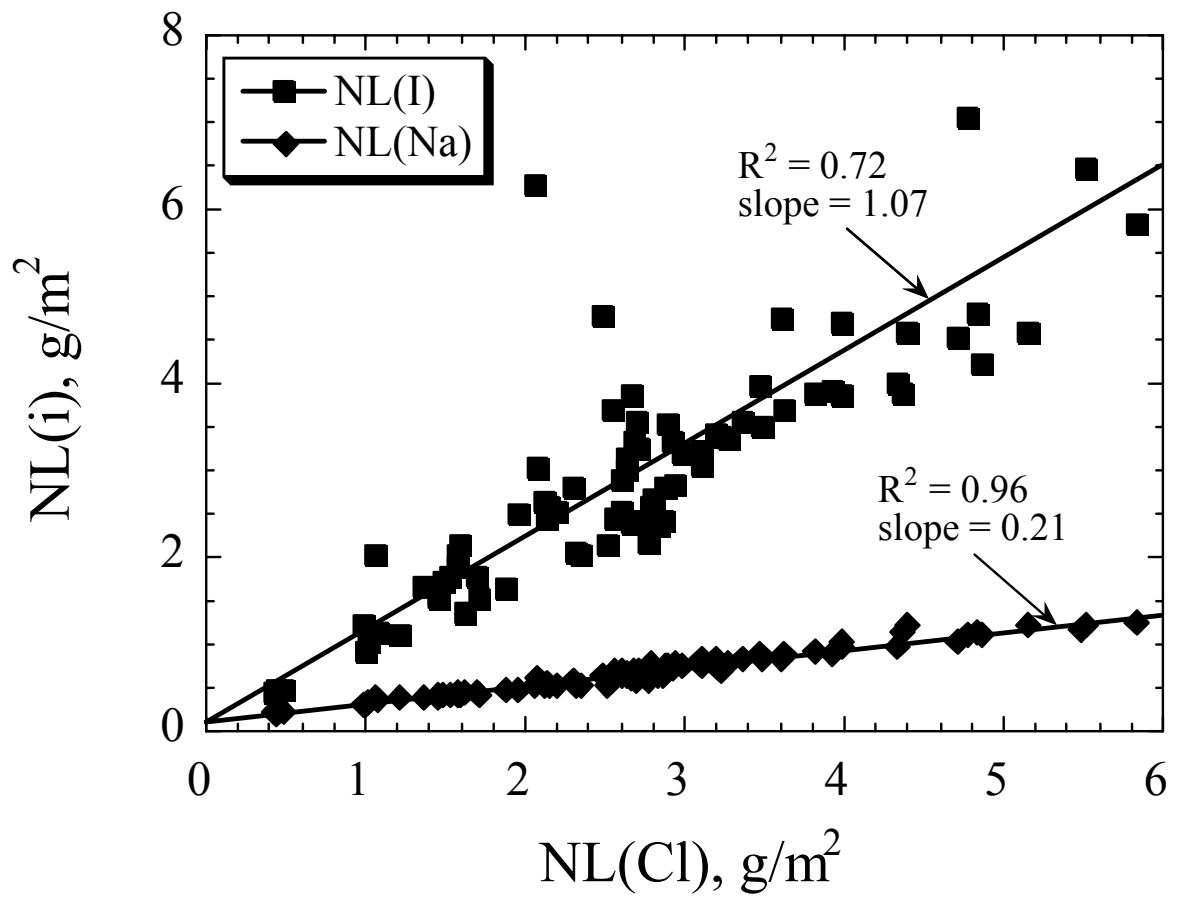

Fig. 28. Correlation between $N L(\mathrm{Cl}), N L(\mathrm{I})$, and $N L(\mathrm{Na})$ in RWS Fraction. 


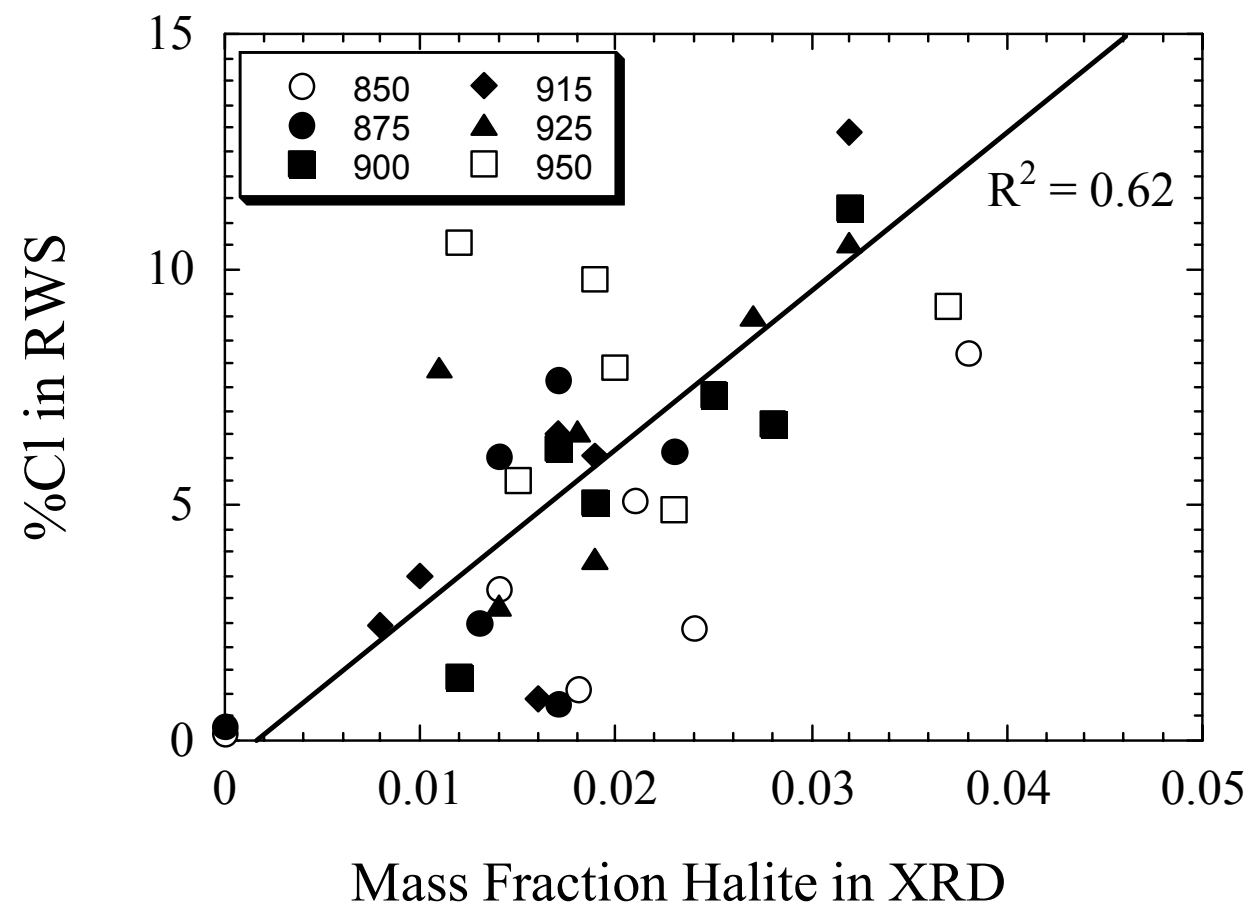

Fig. 29. Correlation between $\% \mathrm{Cl}$ in RWS and Mass $\%$ Halite Measured by XRD for CWF Made at 850, 875, 900, 915, 925, and $950^{\circ} \mathrm{C}$ for Different Hold Times. 


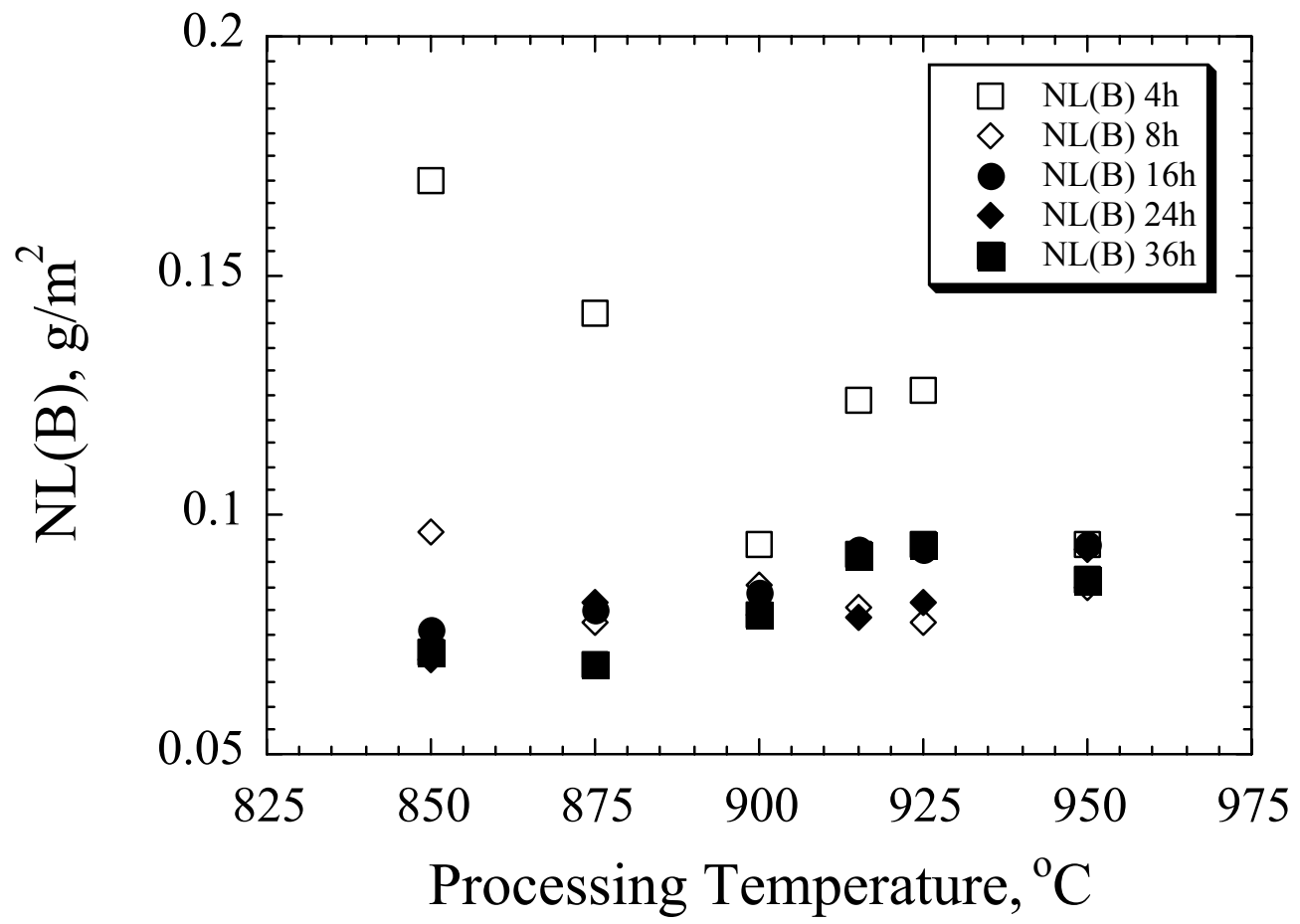

(a)

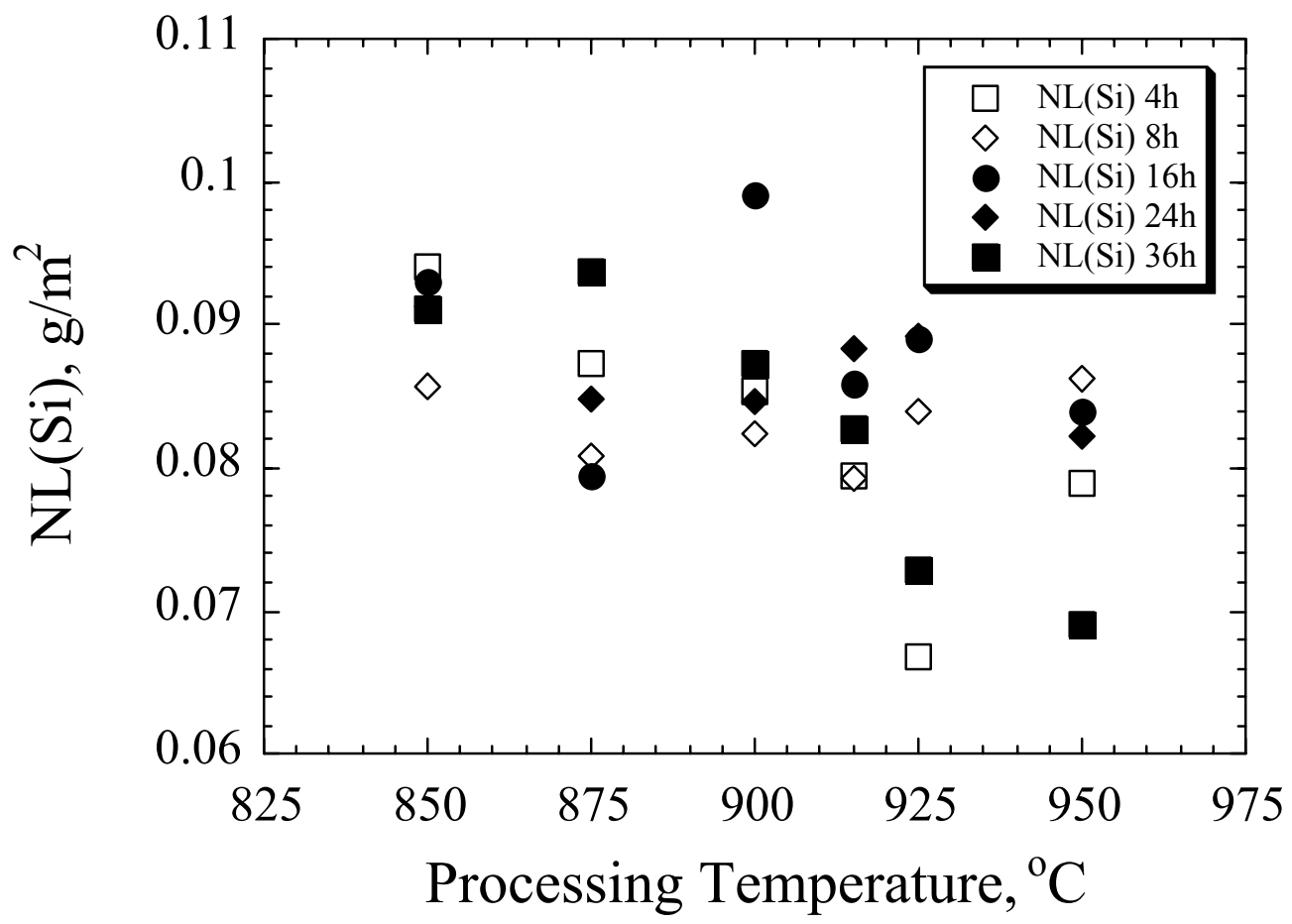

(b)

Fig. 30. PCT Results for T-t PC CWF Materials Made With Different Hold Times: (a) $N L(\mathrm{~B}),($ b) $N L(\mathrm{Si}),(\mathrm{c}) N L(\mathrm{Cl})$ and (d) $N L(\mathrm{Na})$. 


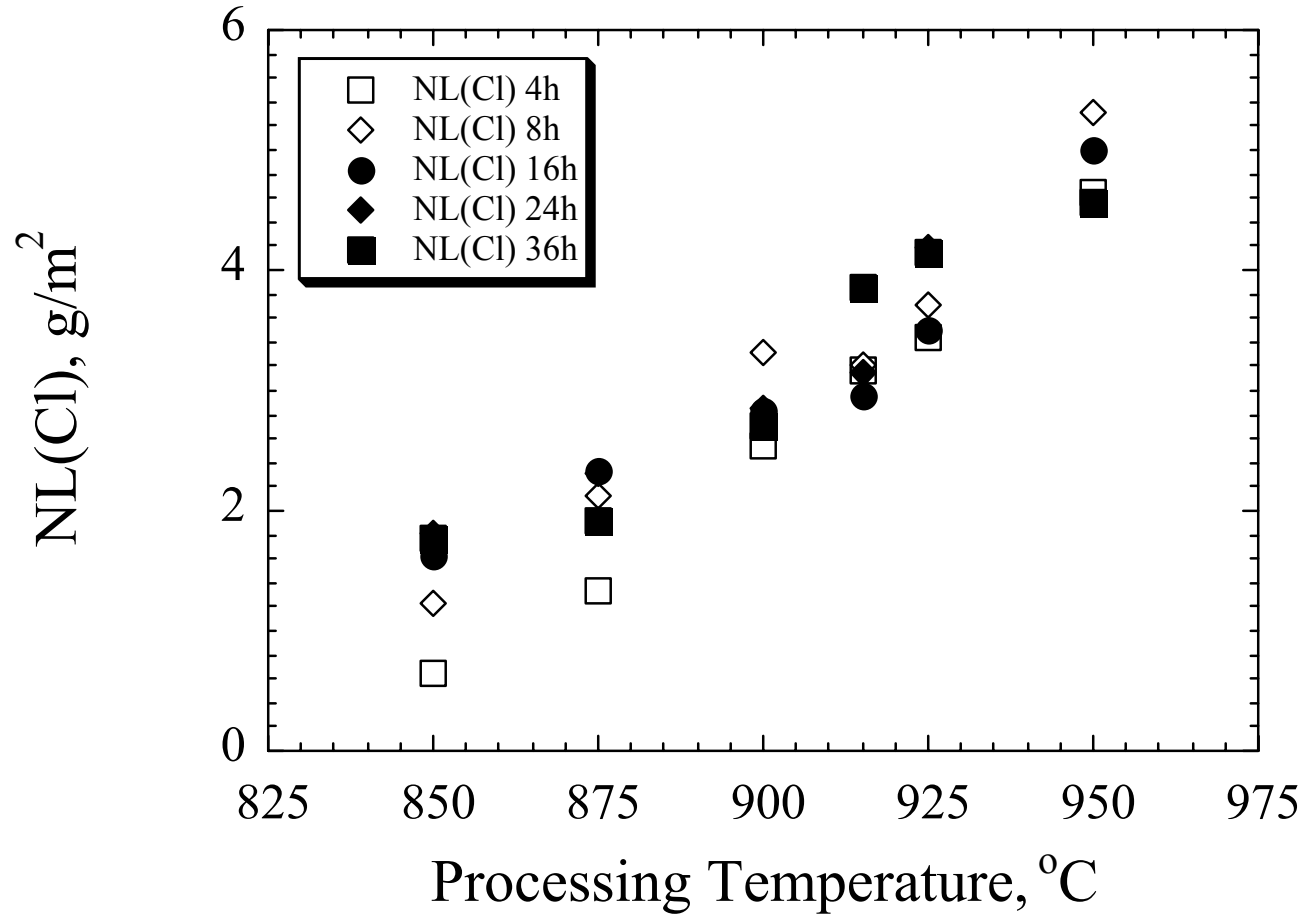

(c)

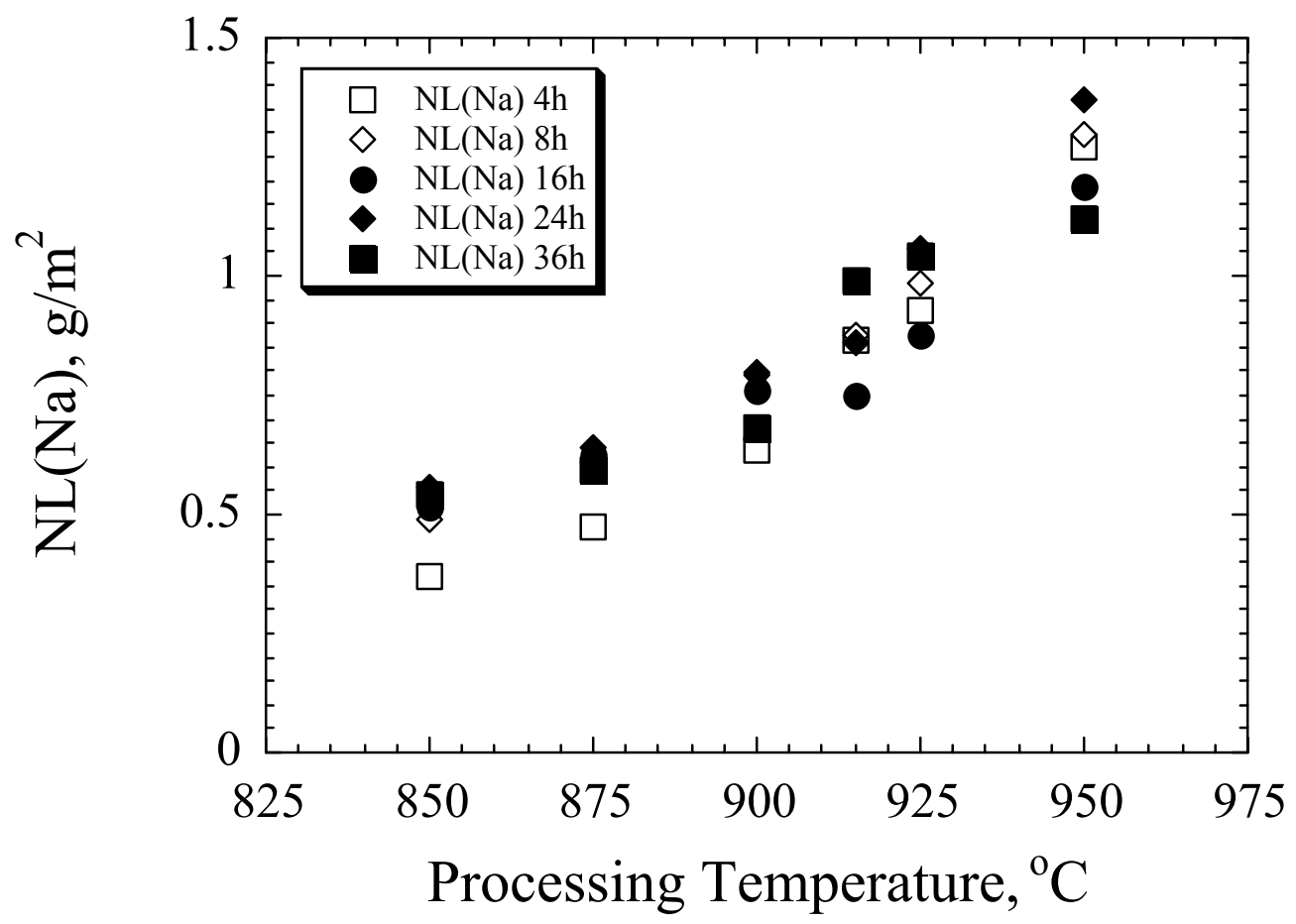

Fig. 30. (Contd.)

(d) 
Internal (Printed and Electronic copies):

T. L. Barber

N. L. Dietz

W. L. Ebert (25)

T. H. Fanning

Internal (Printed Copy Only):

V. A. Davis

M. J. Steindler

S. K. Zussman (2)

Internal (Electronic Copy Only):

\section{J. P. Ackerman}

C. H. Adams

R. K. Ahluwalia

S. E. Aumeier

A. J. Bakel

K. J. Bateman

T. J. Battisti

T. H. Bauer

R. W. Benedict

A. R. Brunsvold

D. B. Chamberlain

Y. I. Chang

J. C. Cunnane

S. S. Cunningham

K. B. Davies

R. N. Elliot

R. A. Evans

R. J. Finch

J. K. Fink

J. A. Fortner

E. K. Fujita

H. E. Garcia

E. C. Gay
K. M. Goff

M. C. Hash

A. S. Hebden

S. Y. Jeong
S. G. Johnson

M. A. Lewis (10)

T. P. O'Holleran
H. F. McFarlane

L. Nuñez

R. L. Parks

D. R. Pedersen

R. D. Phipps

C. L. Pope

M. C. Regalbuto

R. H. Rigg

J. M. Runge

S. R. Sherman

M. F. Simpson

C. T. Snyder

G. M. Teske

Y. Tsai

D. Vaden

G. F. Vandegrift III

D. L. Whalquist

B. R. Westphal

R. A. Wigeland

M. A. Williamson

J. L. Willit

T. L. Wright 


\section{External (Electronic Copy Only):}

M.A. Buckley, ANL-E Library

E. S. Sackett, ANL-W Library

Chemical Technology Review Committee Members:
H. U. Anderson
R. A. Greenkorn
C. Hussey
J. R. Selman
J. S. Tulenko
M. Koch
V. Roan 